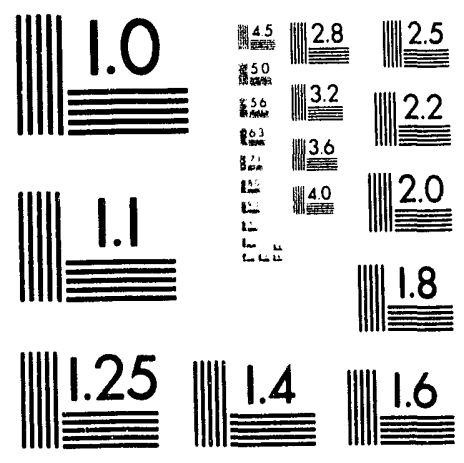



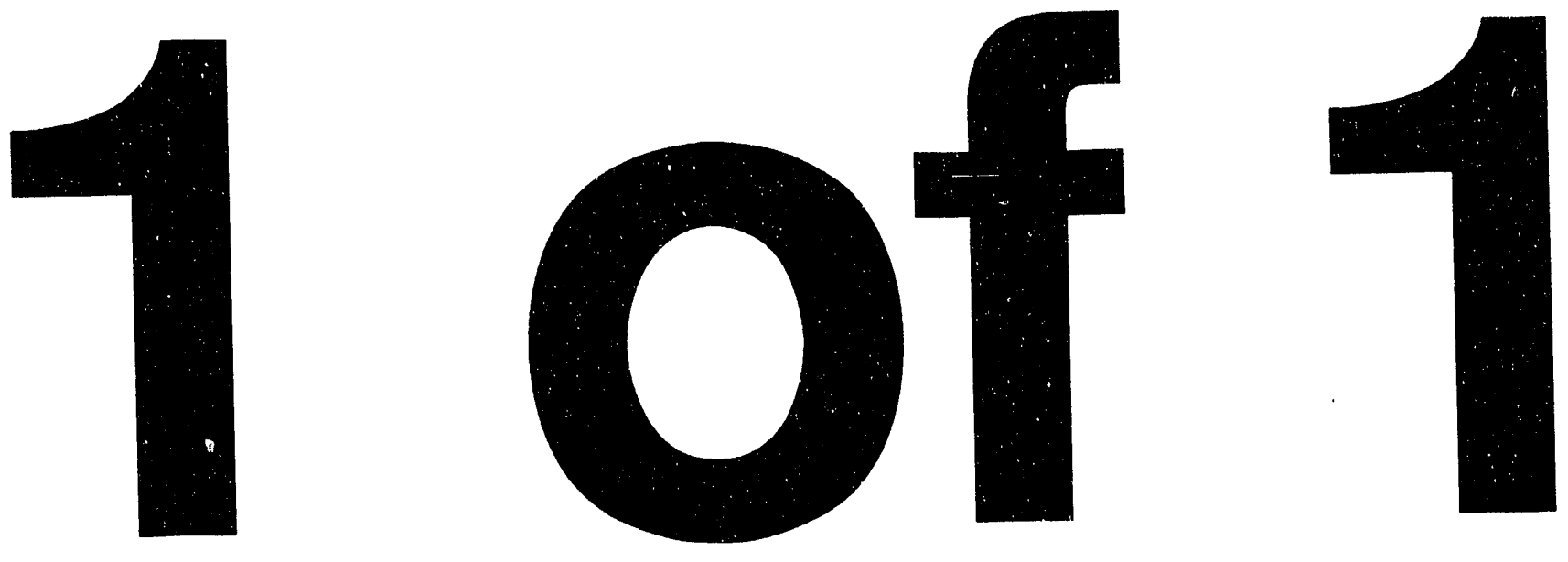


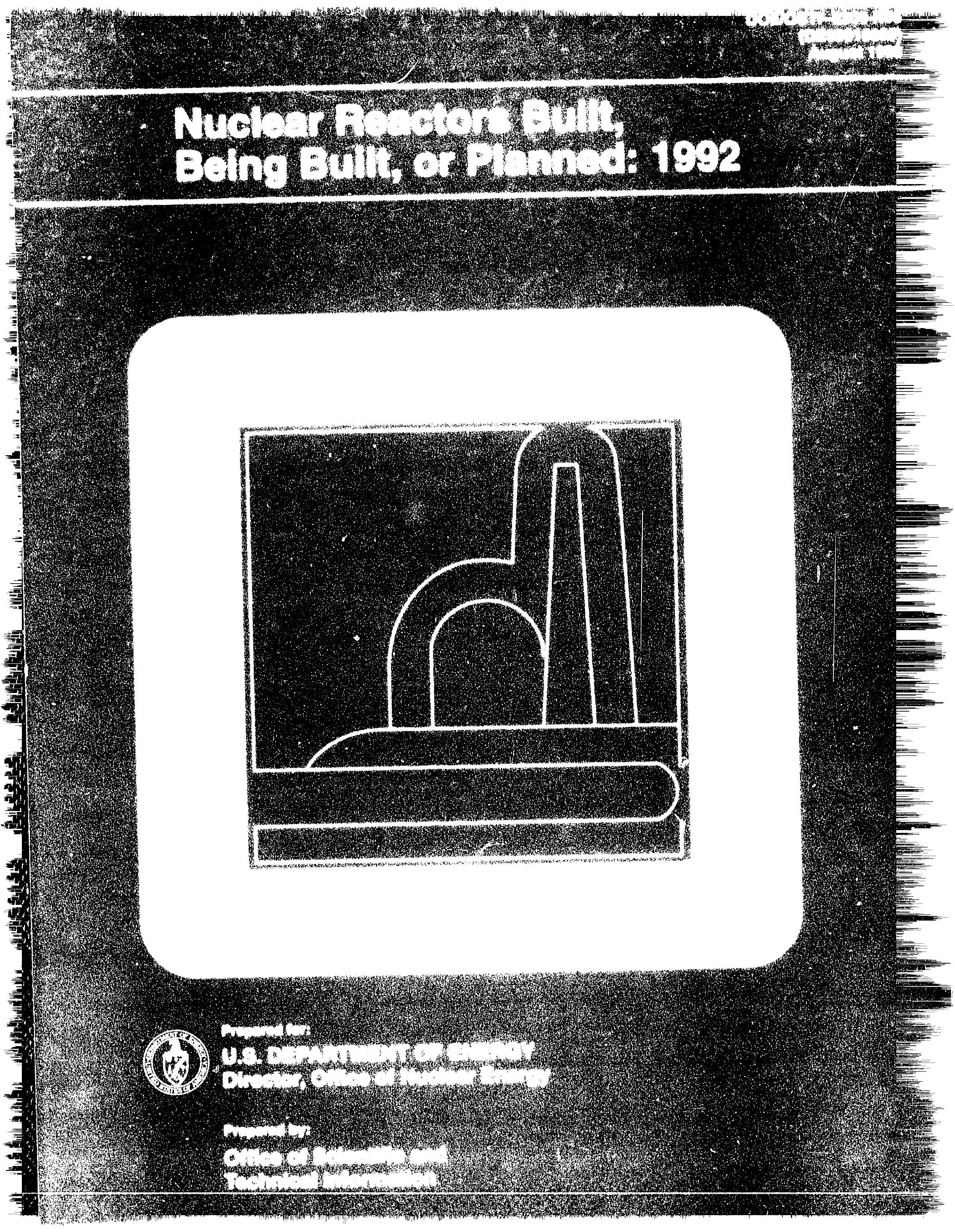


Available to DOE and DOE contractors from the Office of Scientific and Technical Information, P.O. Box 62, Oak Ridge, Tennessec 37831; (615) 576-8401.

Available to the public from the National Technical Information Service, U.S. Department of Commerce, 5285 Port Royal Rd., Springficld, Virginia 22161; (703) $487-4650$. 


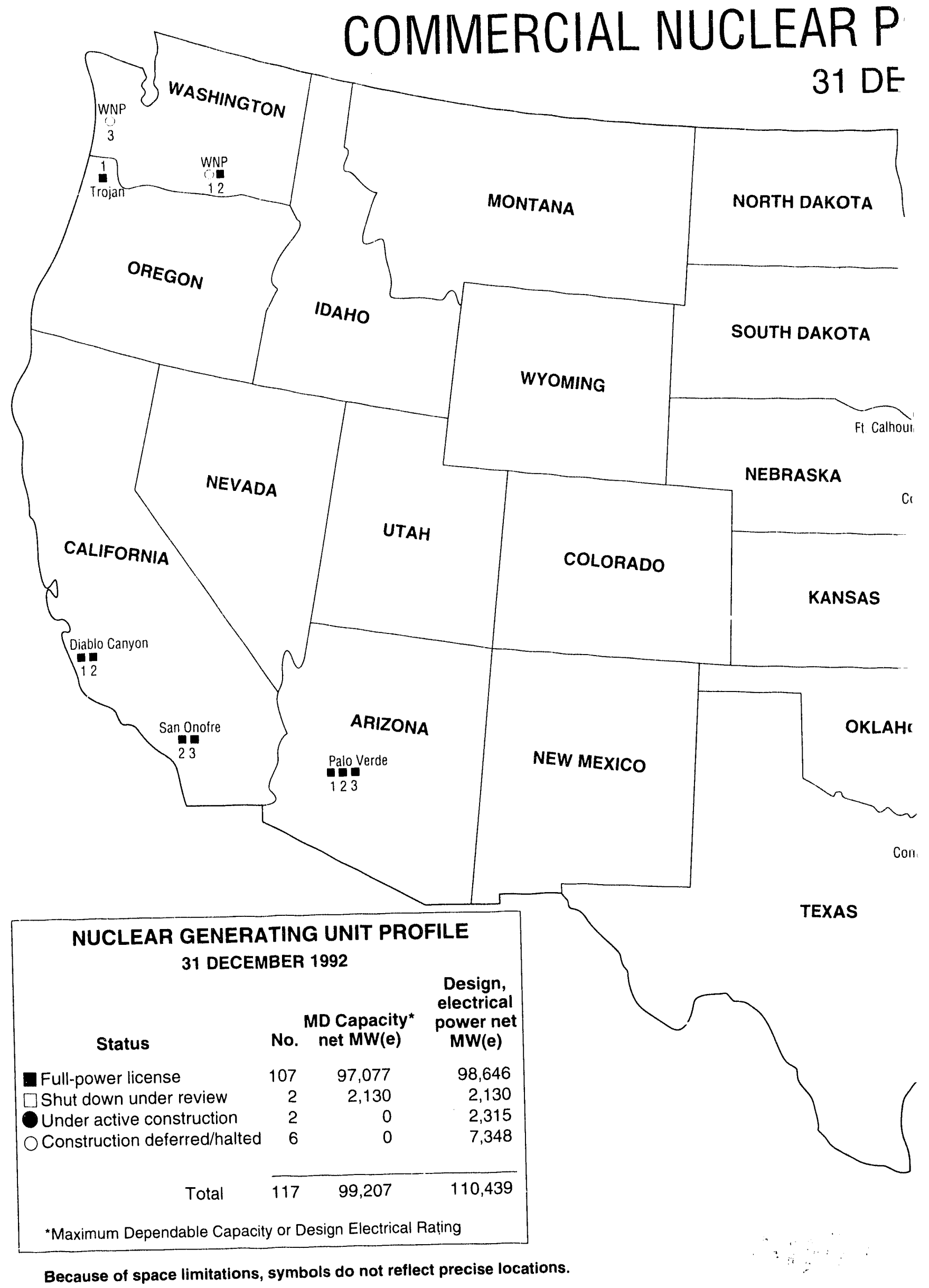




\section{NER REACTORS In THE UNITED STATES}

\section{MBER 1992}

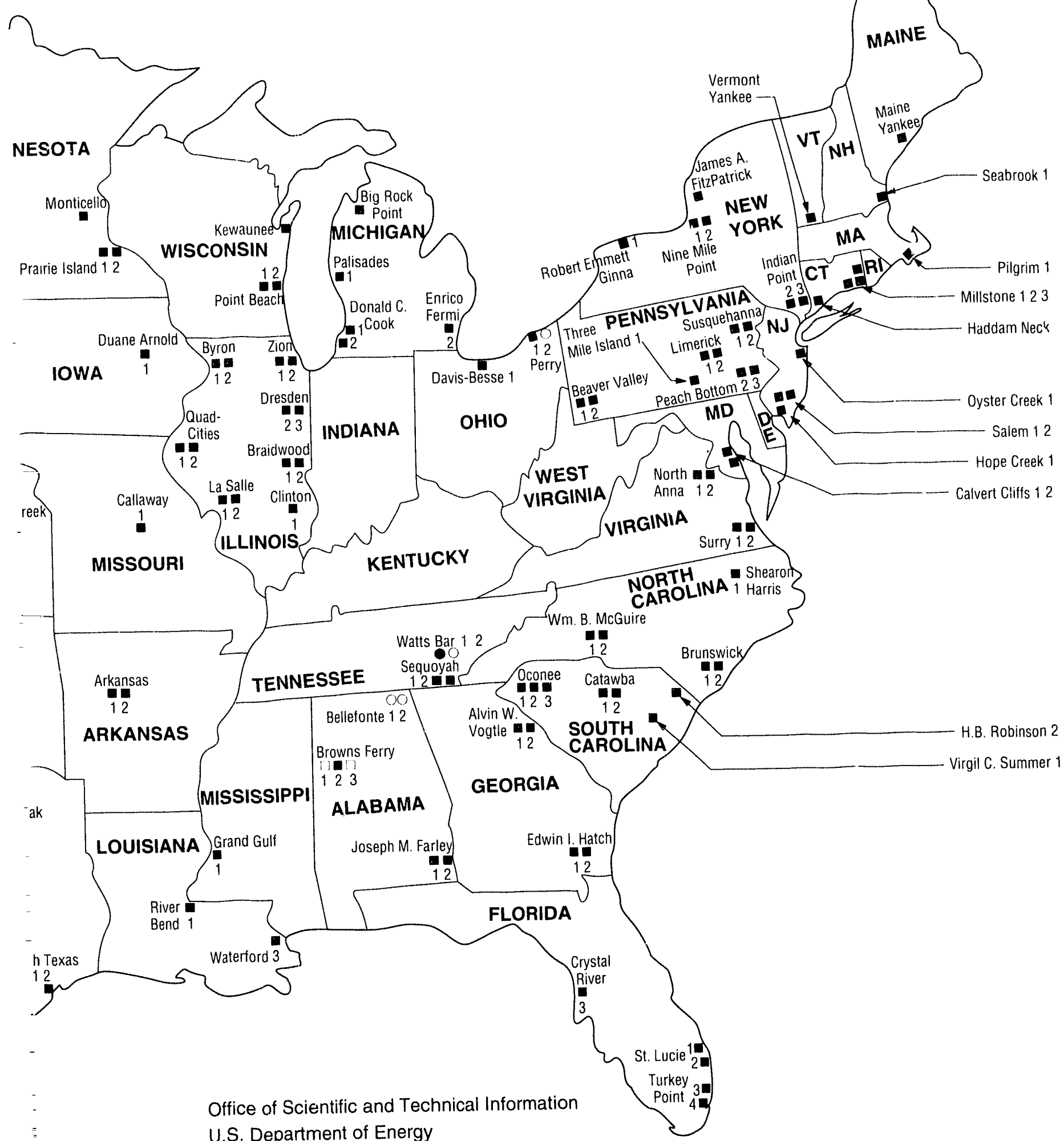

U.S. Department of Energy

From Nuclear Reactors Built, Being Built, or Planned (DOE/OSTI--8200-R56) 
COMMERCIAL NUCLEAR POWEF、 REAC

\begin{tabular}{|c|c|c|c|c|c|c|c|c|c|c|c|c|c|}
\hline Site & Plani name & Status & $\begin{array}{c}\text { MD } \\
\text { capacity. } \\
M W(e)\end{array}$ & $\begin{array}{l}\text { Design. } \\
\text { electrical } \\
\text { power net } \\
M W(e)\end{array}$ & Licensee & Startup & Site & Plant name & Status & $\begin{array}{c}M D \\
\text { rapacity. } \\
M W(e)\end{array}$ & $\begin{array}{c}\text { Design. } \\
\text { electrical } \\
\text { power net } \\
M W(e)\end{array}$ & Licensee & startus \\
\hline ALABAMA & & & & & & & Cortova & $\begin{array}{l}\text { Qutd-cities Station } \\
\text { Une? ? }\end{array}$ & FPl. & 769 & 789 & Communvereatith & $7 \ddot{v a}$ \\
\hline [04:4 $71,1,1$ & $\begin{array}{l}\text { Browns ferry Nucleadt } \\
\text { Power Station Unit i }\end{array}$ & 'SDUH & 1055 & 1065 & 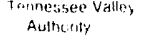 & 308 & Morrs & $\begin{array}{l}\text { Und ? } \\
\text { Dresden Nucleat Power }\end{array}$ & FPl. & $\because 2$ & 794 & $\begin{array}{l}\text { Edison Co } \\
\text { Commonweath }\end{array}$ & 7001 \\
\hline Decatur & $\begin{array}{l}\text { Brownts Ferry Pucleat } \\
\text { Pawer Staturn Unil? }\end{array}$ & FF' & 1.065 & 1065 & $\begin{array}{l}\text { Tennessee Valley } \\
\text { Authorlly }\end{array}$ & 7407 & Morrs & $\begin{array}{l}\text { Station Unit? } \\
\text { Dressden Nurlear Power }\end{array}$ & FPI. & 773 & 294 & $\begin{array}{l}\text { Edison co } \\
\text { Cornmonwealth }\end{array}$ & $71 r 1$ \\
\hline Wrecitur & $\begin{array}{l}\text { Brownst enty Nucipar } \\
\text { Powet Staton Unil } 3\end{array}$ & SDUR & $1,06,5$ & 1065 & $\begin{array}{l}\text { Tẹnnessee Valley } \\
\text { Authority }\end{array}$ & $7 \in 0$ 0 & Seriecia & $\begin{array}{l}\text { Station Unit } 3 \\
\text { La Salle Count Staton. }\end{array}$ & FPL. & 1.036 & 1.078 & $\begin{array}{l}\text { Ésison Co } \\
\text { Comunonwatilt }\end{array}$ & 8206 \\
\hline Wothath & 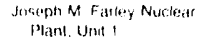 & FFY. & 825 & 829 & $\begin{array}{l}\text { Southern Nucleat } \\
\text { Operaluny Co }\end{array}$ & 7708 & Seneca & $\begin{array}{l}\text { Unut } 1 \\
\text { La Salle County Station. }\end{array}$ & FPl. & 1.036 & 1.078 & $\begin{array}{l}\text { Edsonco } \\
\text { Commonivealth }\end{array}$ & 8403 \\
\hline Dottian & $\begin{array}{l}\text { Josepti M Farley Nurteas } \\
\text { Plant Unt? }\end{array}$ & $F P$ & 624 & 829 & $\begin{array}{c}\text { Southem Nucleat } \\
\text { Operating Co }\end{array}$ & 8105 & $210 n$ & $\begin{array}{l}\text { Unu? } \\
\text { Zwon Nuclear Plant. }\end{array}$ & $\mathrm{FPl}$ & 1040 & 1.040 & $\begin{array}{l}\text { Erlison Co } \\
\text { Commonwealth }\end{array}$ & 7306 \\
\hline sicontsbero & Bellelonte Nuilear Plant & $\mathrm{CDH}$ & & 1.235 & Tennessee valley & indet & 2ता & Unis 1 & & 1.040 & (1.0411 & Edison Co & 1300 \\
\hline Sirntisboro & $\begin{array}{l}\text { Unit } 1 \\
\text { Brothetonte Nucleat Plant. }\end{array}$ & $\mathrm{COH}$ & & 1.235 & $\begin{array}{l}\text { Aufturinly } \\
\text { Temnessiee Valley }\end{array}$ & Indef & Zion & $\begin{array}{l}\text { Z1.0n Nucleat Plani. } \\
\text { Unut? }\end{array}$ & $\mathrm{FPL}$ & 1040 & 1.040 & $\begin{array}{l}\text { Conmor, wealin } \\
\text { Eidison Co }\end{array}$ & 7312 \\
\hline & Unt? & & $\ldots$ & - & Aulhorly & & & & & & & & \\
\hline Tolat & & & 4,844 & 7.323 & & & Tolal & & & 12.683 & 12.815 & & \\
\hline AR:ZONA & & & & & & & IOWA & & & & & & \\
\hline Wente:'sburg & $\begin{array}{l}\text { Palo Verde Nucleat } \\
\text { Gonerating Station }\end{array}$ & FPL & 1.221 & 1.270 & $\begin{array}{l}\text { Anjonia Public } \\
\text { Service Co }\end{array}$ & 8505 & Palo & $\begin{array}{l}\text { Duane Arnold Energy } \\
\text { Center. Unil } 1\end{array}$ & FPL & 515 & 538 & $\begin{array}{l}\text { Iowa Electric } \\
\text { Light \& Power Co }\end{array}$ & 7403 \\
\hline Wintersburg & $\begin{array}{l}\text { IJrit 1 } \\
\text { Palo Verde Nuclear } \\
\text { Generaturn Station. }\end{array}$ & FPL & 1.221 & 1270 & $\begin{array}{l}\text { Arzona Public } \\
\text { Service Co }\end{array}$ & 8604 & tolat & & & 515 & 538 & & \\
\hline Wintersbury & $\begin{array}{l}\text { Unut ? } \\
\text { Palo Verde Nucledr } \\
\text { Goneratum Station }\end{array}$ & FPL & 1.304 & 1.270 & $\begin{array}{l}\text { Arizona Public: } \\
\text { Service Co }\end{array}$ & 87,0 & $\begin{array}{l}\text { KANSAS } \\
\text { Burlington }\end{array}$ & $\begin{array}{l}\text { Wolf Creek Generating } \\
\text { Station }\end{array}$ & FPL & 1128 & 1,170 & $\begin{array}{c}\text { Kansas Gas \& } \\
\text { Electric Co }\end{array}$ & 850 \\
\hline & Un: 3 & & $-\infty$ & $\ldots$ & & & Tolal & & & $1+28$ & 1.170 & & \\
\hline Total & & & 3746 & 3810 & & & LOUISIANA & & & & & & \\
\hline $\begin{array}{l}\text { ARKANSAS } \\
\text { Fussettlille: }\end{array}$ & & & & & & & Si Francisville & $\begin{array}{l}\text { Piver Bend Station, } \\
\text { Unit ! }\end{array}$ & FPL & 936 & 936 & $\begin{array}{l}\text { Gull State Utilties } \\
\text { Co }\end{array}$ & 8511 \\
\hline & $\begin{array}{l}\text { Arkansas Nuclear One } \\
\text { Unit I }\end{array}$ & FPL & 836 & 850 & $\begin{array}{l}\text { Entergy Operations } \\
\text { Inc. }\end{array}$ & 7408 & Tatl & $\begin{array}{l}\text { Waterford Generating } \\
\text { Slation. Un! } 3\end{array}$ & FPL & 1.075 & 1.104 & $\begin{array}{l}\text { Entergy Operations } \\
\text { inc }\end{array}$ & $850^{\circ}$ \\
\hline Rlusseliville & $\begin{array}{l}\text { Aikdnsas Nucleat One. } \\
\text { Unit? }\end{array}$ & FPl & 858 & 912 & $\begin{array}{l}\text { Entergy Operalions. } \\
\text { inc }\end{array}$ & 7812 & tolis 1 & & & 2.011 & 2.040 & & \\
\hline Sotat & & & 1694 & 1.762 & & & MAINE & & & & & & \\
\hline CALIFORNIA & & & & & & & Wiscasset & $\begin{array}{l}\text { Marre ratkes Atomic } \\
\text { Power Plont }\end{array}$ & FPL & 810 & 825 & $\begin{array}{l}\text { Maine Yankee } \\
\text { Alomic Power Co }\end{array}$ & 721 \\
\hline Diabo Canyon & $\begin{array}{l}\text { Diablo Canyon Nucleat } \\
\text { Power Plant Unit } 1\end{array}$ & FPL & 1073 & 1.086 & $\begin{array}{l}\text { Pachilic Gias } 8 \\
\text { Electuc Co }\end{array}$ & 8404 & & & & D & - & & \\
\hline Mablo Canyon & $\begin{array}{l}\text { Diabio Canyon Nuslear } \\
\text { Power Plant Unil ? }\end{array}$ & FPl. & 1.079 & 1119 & $\begin{array}{l}\text { Pacific Gas \& } \\
\text { tieciric Co }\end{array}$ & 8508 & MARYLAND & & & B10 & 825 & & \\
\hline Sin Clemente & $\begin{array}{l}\text { San Onohe Nucleat } \\
\text { Generating Station } \\
\text { Unit? }\end{array}$ & FPL & 1070 & 1070 & $\begin{array}{l}\text { Southern Californa } \\
\text { Edison }\end{array}$ & 8207 & $\begin{array}{l}\text { MARYLAND } \\
\text { L.USEY }\end{array}$ & $\begin{array}{l}\text { Catven Clits: Nuclear } \\
\text { Powet Plant Unt } 1\end{array}$ & FPL & 825 & 845 & $\begin{array}{l}\text { Baltumore Gas \& } \\
\text { Electur, Co }\end{array}$ & 741 \\
\hline San Clemente & $\begin{array}{l}\text { San Ondre Nucleat } \\
\text { Generaling Station. } \\
\text { Un: } 3\end{array}$ & FPL & 1.080 & 1.080 & $\begin{array}{l}\text { Southern Calitornir } \\
\text { Edisun }\end{array}$ & 8308 & Lusby & $\begin{array}{l}\text { Calvert Chifts Nuclear } \\
\text { Power Plant, Lint? }\end{array}$ & $\mathrm{FPL}$ & 825 & 845 & $\begin{array}{c}\text { Baltimore Gas \& } \\
\text { Electric Co }\end{array}$ & 761 \\
\hline Tolat & & & 4.302 & 4.355 & & & Total & & & 1.650 & 1.690 & & \\
\hline CONNECTICUT & & & & & & & MASSACHUSETTS & & & & & & \\
\hline Haddam Neck & Hiaddam Nech Ptanl & $F P_{L}$ & 569 & $58 ?$ & $\begin{array}{l}\text { Connechicul Yankee } \\
\text { Alomic Power } \mathrm{Cn}\end{array}$ & 6707 & Plymouth & $\begin{array}{l}\text { Pilgrim Nuclear Power } \\
\text { Station. Unil I }\end{array}$ & FPL & 670 & 655 & Boston Edison Co. & 721 \\
\hline Waterord & $\begin{array}{l}\text { Milistone Nucleat Power } \\
\text { Station Unit } 1\end{array}$ & $F P_{L}$ & 654 & 660 & $\begin{array}{l}\text { Northeast Nuclear } \\
\text { Energy Co }\end{array}$ & 7010 & Total & & & 670 & 655 & & \\
\hline Watertord & $\begin{array}{l}\text { Millstone Nuclear Fower } \\
\text { Station. Unit? }\end{array}$ & $F P L$ & 857 & 870 & $\begin{array}{l}\text { Nonheasi Nucleas } \\
\text { Energy Co }\end{array}$ & 7510 & $\begin{array}{l}\text { MICHIGAN } \\
\text { Big ROCK PoIn! }\end{array}$ & Big Pock Point & $\mathrm{FPL}$ & 69 & 72 & Consumers Pawer & 62 \\
\hline Total & & & 3.222 & 3266 & & & Bridgman & $\begin{array}{l}\text { Dorrald C Cook Nuclear } \\
\text { Power Plant. Unit? }\end{array}$ & $\mathrm{FPL}$ & 1.060 & 1100 & $\begin{array}{l}\text { Indiana and Michigan } \\
\text { Electric Co }\end{array}$ & 78 \\
\hline $\begin{array}{l}\text { FLORIDA } \\
\text { Finrdia Cily }\end{array}$ & Turkey Point Plant & FPL & 666 & 693 & Flonda Power \& & 7210 & Newport & $\begin{array}{l}\text { Enrrico Fermi Alomic } \\
\text { Power Plant. Unil } 2\end{array}$ & FPL & 1.093 & 1.093 & Detroll Edison Co & 85 \\
\hline Flonda Cily & $\begin{array}{l}\text { Unit } 3 \\
\text { rurkey Poont Plant } \\
\text { Unit a }\end{array}$ & $F P L$ & 566 & 693 & $\begin{array}{l}\text { Light Co } \\
\text { Florida Power \& } \\
\text { Light Co }\end{array}$ & 7306 & South Haven & $\begin{array}{l}\text { Palisades Nucleat } \\
\text { Plant. Unt } 1\end{array}$ & FPL & 730 & 805 & $\begin{array}{l}\text { Consumers Power } \\
\text { Co }\end{array}$ & 11 \\
\hline Fon Plerce & $\begin{array}{l}\text { 5) Lucie Plant. } \\
\text { Unil: }\end{array}$ & FPL & 839 & 830 & $\begin{array}{l}\text { Florida Power \& } \\
\text { Light Co }\end{array}$ & 7604 & Tolal & & & 3.972 & 4.100 & & \\
\hline Fort fierce & $\begin{array}{l}\text { Si Lucie Plani } \\
\text { Uimt? }\end{array}$ & FPL & 839 & 830 & $\begin{array}{l}\text { Fiorida Power \& } \\
\text { Light Ca }\end{array}$ & 8306 & MINNESOTA & & & & & & \\
\hline Red Level & $\begin{array}{l}\text { Crystal River Nuclear } \\
\text { Plant Unt } 3\end{array}$ & FPL & 821 & 825 & $\begin{array}{l}\text { Flonda Power } \\
\text { Corp }\end{array}$ & .7701 & Monticello & $\begin{array}{l}\text { Monticello Nuclear } \\
\text { Generaling Plant }\end{array}$ & FPL & 536 & 545 & $\begin{array}{l}\text { Northern States } \\
\text { Power Co }\end{array}$ & 10 \\
\hline Tutal & & & 3.831 & 3871 & & & Fed Wing & $\begin{array}{l}\text { Pramie Island Nuclear } \\
\text { Generating Plant. } \\
\text { Unit } 1\end{array}$ & FPL & 503 & 530 & $\begin{array}{l}\text { Northern Slates } \\
\text { Power Co }\end{array}$ & 7312 \\
\hline $\begin{array}{c}\text { GEORGIA } \\
\text { Baxlk'y }\end{array}$ & $\begin{array}{l}\text { Edwan I Hatch Nuclear } \\
\text { Plan! Unil ? }\end{array}$ & FPL & 750 & 776 & $\begin{array}{l}\text { Cieorga Power } \\
\text { Cor }\end{array}$ & 7409 & Red Wing & $\begin{array}{l}\text { Prarrie Island Nuclear } \\
\text { Generating Plant } \\
\text { Unt } 2\end{array}$ & $\mathrm{FPL}$ & 500 & 530 & $\begin{array}{l}\text { Northern Stales } \\
\text { Power Co }\end{array}$ & 7412 \\
\hline Baxley & $\begin{array}{l}\text { Edwin I Hatch Nuclear } \\
\text { Ptant Unt ? }\end{array}$ & FPL. & 761 & 784 & $\begin{array}{l}\text { Georyla Power } \\
\text { Co }\end{array}$ & 7807 & Total & & & 1.539 & 1,605 & & \\
\hline Waynestoru & $\begin{array}{l}\text { Alwin } W \text { Vogtle Nuciear } \\
\text { Plant Unit : }\end{array}$ & FPL. & 1.101 & 1101 & $\begin{array}{l}\text { Georga Power } \\
\text { Co }\end{array}$ & 8703 & MISSISSIPPI & & & & & & \\
\hline Wayresboro & $\begin{array}{l}\text { Alvin } w \text { vogite Nuclear } \\
\text { Plant Unin } 2\end{array}$ & FPL & 1.101 & 1101 & $\begin{array}{l}\text { Georgla Power } \\
\text { cc }\end{array}$ & 8903 & Port Gibson & $\begin{array}{l}\text { Grand Gulf Nucleat } \\
\text { Station. Unit } 1\end{array}$ & FPL & 1.142 & 1.250 & $\begin{array}{l}\text { Entergy } \\
\text { Operatons } \\
\text { lnic }\end{array}$ & 8208 \\
\hline Tolal & & & 3.713 & 3.762 & & & Tolal & & & 1.142 & 1.250 & & \\
\hline $\begin{array}{l}\text { ILLINOIS } \\
\text { Braidwoud }\end{array}$ & $\begin{array}{l}\text { Braidwood Station } \\
\text { Unn! }\end{array}$ & FPL & 1.120 & 1,120 & $\begin{array}{l}\text { Commonwealthr } \\
\text { Edison Co }\end{array}$ & 870.5 & $\begin{array}{l}\text { MISSOURI } \\
\text { Fulton }\end{array}$ & Callaway Plant Unis 1 & FPL & $1: 20$ & 1.171 & Union Electric Co & 8410 \\
\hline Btaldwood & $\begin{array}{l}\text { Brardwond Station } \\
\text { Unir? }\end{array}$ & FPL & 1120 & 1120 & $\begin{array}{l}\text { Eommonive allth } \\
\text { Ealson Co }\end{array}$ & 8803 & Total & & & 1.120 & 1.171 & & \\
\hline Byron & $\begin{array}{l}\text { Byron Station. } \\
\text { Unit a }\end{array}$ & FPL & 1129 & 1120 & $\begin{array}{l}\text { Cominonwealth } \\
\text { Earson co. }\end{array}$ & 8502 & NEBRASKA & & & & & & \\
\hline Byron & $\begin{array}{l}\text { Byron Station } \\
\text { Unit? }\end{array}$ & FPL & 1129 & 1120 & $\begin{array}{l}\text { Conmomwealth } \\
\text { Edison Co }\end{array}$ & 8701 & Brownville & $\begin{array}{l}\text { Conper Nuclear } \\
\text { Station }\end{array}$ & $F P L$ & 764 & 778 & $\begin{array}{l}\text { Nebraska Public } \\
\text { Power Districl }\end{array}$ & 7102 \\
\hline Ciniton & $\begin{array}{l}\text { Clinton Power Siation } \\
\text { Unt: }\end{array}$ & $F P$ & 950 & 933 & llinnols Puwer ca & 8702 & Fun Caitroun & $\begin{array}{l}\text { Fon Calnoun } \\
\text { Station Und }\end{array}$ & FPL. & 478 & 478 & $\begin{array}{l}\text { Omatha Pubtic } \\
\text { Power District }\end{array}$ & 7308 \\
\hline Curteva & $\begin{array}{l}\text { Quad Cittes Station } \\
\text { Unm }\end{array}$ & $F P L$ & 769 & 789 & $\begin{array}{l}\text { Commonweilth } \\
\text { Edtiton Co }\end{array}$ & 310 & Tolat & & & $1 \div 42$ & 1.256 & & \\
\hline
\end{tabular}


: IN THE UNITED STATES AS OF 31 DEC 1992

\begin{tabular}{|c|c|c|c|c|c|c|c|c|c|c|c|c|c|}
\hline Site & Plant name & Status & $\begin{array}{c}M D \\
\text { capacity. } \\
M W(\mathbf{e})\end{array}$ & $\begin{array}{l}\text { Design, } \\
\text { electrical } \\
\text { power net } \\
M W(0)\end{array}$ & Licensee & Startup & Site & Plant name & Status & $\begin{array}{c}\text { MD } \\
\text { capacity. } \\
M W(\theta)\end{array}$ & $\begin{array}{l}\text { Design, } \\
\text { electrical } \\
\text { power net } \\
M W(e)\end{array}$ & Licensee & Startup \\
\hline \multirow{3}{*}{$\begin{array}{l}\text { NEW HAMPSHIRE } \\
\text { Seabrook }\end{array}$} & \multirow{5}{*}{$\begin{array}{l}\text { Seabrook Nucloat } \\
\text { Station Init } 1\end{array}$} & \multirow{5}{*}{ FPL. } & \multirow{3}{*}{1.198} & \multirow{3}{*}{1,198} & \multirow{5}{*}{$\begin{array}{l}\text { Publuc Sorvice } \\
\text { Co of New } \\
\text { Hampshire }\end{array}$} & \multirow{4}{*}{8906} & \multicolumn{2}{|c|}{ SOUTH CAROLINA } & & & & & \\
\hline & & & & & & & Hartsville & $\begin{array}{l}\text { H B Robirison Plant. } \\
\text { Unil? }\end{array}$ & FPL & 665 & 700 & $\begin{array}{l}\text { Carolina Power \& } \\
\text { Light Co }\end{array}$ & 7009 \\
\hline & & & & & & & Jenkinsville & $\begin{array}{l}\text { Virgil C. Summer } \\
\text { Nuclear Station, }\end{array}$ & FPL & 885 & 900 & $\begin{array}{l}\text { South Carolina } \\
\text { Electric \& }\end{array}$ & 8210 \\
\hline \multirow[t]{2}{*}{ Total } & & & \multirow[t]{2}{*}{1.198} & \multirow[t]{2}{*}{1.198} & & & & Unit I & & & & Gas Co & \\
\hline & & & & & & \multirow[b]{2}{*}{8606} & Lake Wylte & $\begin{array}{l}\text { Catawba Nuclear } \\
\text { Station. Unit } 1\end{array}$ & FPL & 1.145 & 1.145 & Duke Power Co. & 8501 \\
\hline \multirow[t]{2}{*}{ Salem } & \multirow{2}{*}{$\begin{array}{l}\text { Hope Creek Nuciear } \\
\text { Generaling Slation. } \\
\text { Unil } 1\end{array}$} & \multirow[t]{2}{*}{ FPi. } & \multirow[t]{2}{*}{1,067} & \multirow[t]{2}{*}{1,067} & $\begin{array}{l}\text { Public Senvice } \\
\text { Electric \& }\end{array}$ & & Lake Wylie & $\begin{array}{l}\text { Catawba Nuclear } \\
\text { Station. Unit } 2\end{array}$ & FPL & 1.145 & 1.145 & Duke Power $\mathrm{Co}$ & 8605 \\
\hline & & & & & $\begin{array}{c}\text { Gas Co } \\
\text { Public Sorvice }\end{array}$ & 7612 & Seneca & $\begin{array}{l}\text { Oconee Nuclear } \\
\text { Station, Unit } 1\end{array}$ & FPL & 860 & 887 & Duke Power Co & 7304 \\
\hline Salem & $\begin{array}{l}\text { Satern Nuclear } \\
\text { Generatung Station. } \\
\text { Unit r }\end{array}$ & ret & 1,100 & 1.17s & $\begin{array}{l}\text { Electric \& } \\
\text { Gas Co. }\end{array}$ & & Seneca & $\begin{array}{l}\text { Oconee Nucleat } \\
\text { Station, Unit ? }\end{array}$ & FPL & 860 & 887 & Duke Power Co. & 7311 \\
\hline Salem & $\begin{array}{l}\text { Salem Nuclear } \\
\text { Ganeraling Slation. } \\
\text { Unit } 2\end{array}$ & FPL & 1.106 & 1,115 & $\begin{array}{l}\text { Public Service } \\
\text { Electric \& } \\
\text { Gas Co. }\end{array}$ & 8008 & Seneca & $\begin{array}{l}\text { Oconee Nuclear } \\
\text { Station, Unit } 3\end{array}$ & FPL & 860 & 887 & Duke Power Co. & 7409 \\
\hline Tams River & $\begin{array}{l}\text { Oyster Creek Nuclear } \\
\text { Power Plant. Unit } 1\end{array}$ & FPL & 620 & 650 & $\begin{array}{l}\text { GPU Nucleat } \\
\text { Corp }\end{array}$ & 6905 & TENUFCOEF & & & 6.420 & 6.551 & & \\
\hline Total & & & 3,899 & 3.947 & & & $\begin{array}{l}\text { TENNESSEE } \\
\text { DaISY }\end{array}$ & $\begin{array}{l}\text { Sequoyan Nuclear } \\
\text { Plant. Unit } 1\end{array}$ & $\mathrm{FPL}$ & 1.148 & 1.148 & $\begin{array}{l}\text { Tennessee Valtey } \\
\text { Autharily }\end{array}$ & 8007 \\
\hline $\begin{array}{l}\text { NEW YORK } \\
\text { Buchanan }\end{array}$ & Indian Poin! Slation, & FPL & 849 & 873 & Consolidated & 7305 & Daisy & $\begin{array}{l}\text { Sequoyah Nucleat } \\
\text { Plant. Unit } 2\end{array}$ & FPL & 1.148 & 1.148 & $\begin{array}{l}\text { Tennessee Valley } \\
\text { Authorily }\end{array}$ & 8111 \\
\hline & Unit 2 & & & & $\begin{array}{l}\text { Edisun Co of } \\
\text { New York. Inc. }\end{array}$ & & Spring City & $\begin{array}{l}\text { Walls Bar Nuclear } \\
\text { Plant. Unit } 1\end{array}$ & uc & & 1.165 & $\begin{array}{l}\text { Tennesseo Vailey } \\
\text { Authority }\end{array}$ & 9312 \\
\hline Buchanan & $\begin{array}{l}\text { Indian Point Station. } \\
\text { Unit } 3\end{array}$ & FPL & 1,000 & 965 & $\begin{array}{l}\text { New York Power } \\
\text { Authority }\end{array}$ & 7604 & Spring Cily & $\begin{array}{l}\text { Watts Bar Nuclear } \\
\text { Plant, Unit } 2\end{array}$ & $\mathrm{CDH}$ & 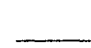 & 1,165 & $\begin{array}{l}\text { Tennesser Valley } \\
\text { Authority }\end{array}$ & Indel \\
\hline Ontario & $\begin{array}{l}\text { Roben Emment Ginna } \\
\text { Nuctear Power } \\
\text { Plant, Unut } 1\end{array}$ & FPL & 470 & 470 & $\begin{array}{l}\text { Rochesler Gas \& } \\
\text { Electric Corp. }\end{array}$ & 6911 & Tolal & & & 2.296 & 4.626 & & \\
\hline Scriba & $\begin{array}{l}\text { James A FirzPatrick } \\
\text { Nuclear Power } \\
\text { Plant }\end{array}$ & FPL & 794 & 816 & $\begin{array}{l}\text { New York Power } \\
\text { Authority }\end{array}$ & 7411 & $\begin{array}{l}\text { TEXAS } \\
\text { Bay City }\end{array}$ & $\begin{array}{l}\text { South Texas Project. } \\
\text { Unil } 1\end{array}$ & FPL & 1,250 & 1,250 & $\begin{array}{l}\text { Houston Lighting \& } \\
\text { Power Co. }\end{array}$ & 8803 \\
\hline Scriba & $\begin{array}{l}\text { Nine Mule Point } \\
\text { Nuclear Station. }\end{array}$ & $\mathrm{FPL}$ & 610 & 620 & $\begin{array}{l}\text { Niagara Mohawk } \\
\text { Power Corp. }\end{array}$ & 6909 & Bay City & $\begin{array}{l}\text { South Texas Project. } \\
\text { Unil } 2\end{array}$ & FPL & 1.250 & 1.250 & $\begin{array}{l}\text { Houslon Lighting \& } \\
\text { Power Co. }\end{array}$ & 8902 \\
\hline Scnba & $\begin{array}{l}\text { Nine Mile Point } \\
\text { Nuclear Station. }\end{array}$ & FPL & 1.080 & 1.080 & $\begin{array}{l}\text { Niagara Mohawk } \\
\text { Power Corp }\end{array}$ & 8705 & Glen Rose & $\begin{array}{l}\text { Comanche Peak } \\
\text { Steam Electric } \\
\text { Station, Unit } 1\end{array}$ & FPL & 1.150 & 1,150 & $\begin{array}{l}\text { Texas Ulilities } \\
\text { Genoraling Co. }\end{array}$ & 9004 \\
\hline & Unit 2 & & 4.803 & - & & & Glen Fose & $\begin{array}{l}\text { Comanche Peak } \\
\text { Steam Electric } \\
\text { Station. Unit } 2\end{array}$ & uc & & 1.150 & $\begin{array}{l}\text { Texas Uilities } \\
\text { Generalling Co }\end{array}$ & 9212 \\
\hline NORTH CAROLINA & & & & & & & Total & & & 3,650 & 4.800 & & \\
\hline Bonsal & $\begin{array}{l}\text { Shearon Harris } \\
\text { Nuclear Power }\end{array}$ & $\mathrm{FPL}$ & 915 & 900 & $\begin{array}{l}\text { Cárolina Power \& } \\
\text { Light Co }\end{array}$ & 8701 & $\begin{array}{l}\text { VERMONT } \\
\text { Vernon }\end{array}$ & & & & & & \\
\hline $\begin{array}{l}\text { Cowans Ford } \\
\text { Dam }\end{array}$ & $\begin{array}{l}\text { William B. McGurre } \\
\text { Nuclear Station, } \\
\text { Unit } 1\end{array}$ & FPL & 1.150 & 1.180 & Duke Power Co. & 8108 & Vernon & $\begin{array}{l}\text { Vermont Yankee } \\
\text { Nuclear Power } \\
\text { Station }\end{array}$ & FPL & $\longrightarrow$ & - & $\begin{array}{l}\text { Vermont Yankee } \\
\text { Nuclear Power } \\
\text { Corp. }\end{array}$ & 7203 \\
\hline $\begin{array}{l}\text { Cowans Ford } \\
\text { Dam }\end{array}$ & $\begin{array}{l}\text { William B. McGuire } \\
\text { Nuclear Station, } \\
\text { Unit } 2\end{array}$ & FPL. & 1.150 & 1,180 & Duke Power Co. & 8305 & $\begin{array}{l}\text { Tolal } \\
\text { VIRGINIA }\end{array}$ & & & 504 & 514 & & \\
\hline Southport & $\begin{array}{l}\text { Brunswick Steam } \\
\text { Electric Plant. }\end{array}$ & FPL & 790 & 821 & $\begin{array}{l}\text { Carolina Power \& } \\
\text { Light Co. }\end{array}$ & 7610 & Gravel Neck & $\begin{array}{l}\text { Sury Power Station. } \\
\text { Unil } 1\end{array}$ & FPL & 781 & 788 & $\begin{array}{l}\text { Virginia Electric \& } \\
\text { Power Co. }\end{array}$ & 7207 \\
\hline Soulthport & $\begin{array}{l}\text { Unit 1 } \\
\text { Brunswick Steam } \\
\text { Electic Plant. } \\
\text { Unit } 2\end{array}$ & FPL & 790 & 821 & $\begin{array}{l}\text { Carolina Power } 8 \\
\text { Light Co }\end{array}$ & 7503 & Gravel Neck & $\begin{array}{l}\text { Surry Power Slation. } \\
\text { Unit } 2 \\
\text { North Anna Power } \\
\text { Station. Unit 1 }\end{array}$ & FPL & $\begin{array}{l}781 \\
915\end{array}$ & $\begin{array}{l}788 \\
907\end{array}$ & $\begin{array}{l}\text { Virginia Electric \& } \\
\text { Power Co } \\
\text { Virginia Electric \& } \\
\text { Power Co. }\end{array}$ & $\begin{array}{l}7303 \\
7804\end{array}$ \\
\hline Total & & & 4,795 & 4,902 & & & Mineral & $\begin{array}{l}\text { North Anna Power } \\
\text { Station, Unit } 2\end{array}$ & FPL & 915 & 907 & $\begin{array}{l}\text { Virgina Eleciric \& } \\
\text { Power Co. }\end{array}$ & 8006 \\
\hline $\mathrm{OH}$ & & & & & & & Total & & & 3,392 & 3,390 & & \\
\hline Oak Harbor & $\begin{array}{l}\text { Davis-Besse Nuclear } \\
\text { Power Station, } \\
\text { Unit } 1\end{array}$ & FPL & 860 & 906 & Toledo Edison Co. & 7708 & $\begin{array}{l}\text { WASHINGTON } \\
\text { Richland }\end{array}$ & Washington Nuclear & $\mathrm{CDH}$ & & 1266 & Washirgton Public & Inder. \\
\hline Perry & $\begin{array}{l}\text { Perry Nuclear Power } \\
\text { Plant. Unit } 1\end{array}$ & FPL & 1,205 & 1,205 & $\begin{array}{l}\text { Cleveland Electric } \\
\text { lfluminating Co. }\end{array}$ & 8606 & Richland & $\begin{array}{l}\text { Wroject. Unit 1 } \\
\text { Prod }\end{array}$ & Con & & & $\begin{array}{l}\text { Power Supply } \\
\text { System }\end{array}$ & \\
\hline Perry & $\begin{array}{l}\text { Perry Nuclear Power } \\
\text { Plant. Unit } 2\end{array}$ & $\mathrm{CDH}$ & & 1,205 & $\begin{array}{l}\text { Cleveland Eloctric } \\
\text { Illuminating Co. }\end{array}$ & Indef & Richland & $\begin{array}{l}\text { Washington Nuclear } \\
\text { Project. Unit } 2\end{array}$ & FPL & 1.095 & 1.100 & $\begin{array}{l}\text { Washinglon Public } \\
\text { Power Supply } \\
\text { System }\end{array}$ & $84 \mathrm{Cl}$ \\
\hline Total & & & 2,065 & 3,316 & & & Salsop & $\begin{array}{l}\text { Washington Nuclear } \\
\text { Project. Unit } 3\end{array}$ & $\mathrm{CDH}$ & & 1,242 & $\begin{array}{l}\text { Washinglon Public } \\
\text { Power Supply }\end{array}$ & Indel. \\
\hline $\begin{array}{l}\text { OREGON } \\
\text { Prescoll }\end{array}$ & Trojan Nuclear Planl. & FPL & 1.075 & 1,130 & Portland General & 7512 & & & & - & - & System & \\
\hline & Unit 1 & & $-1+3$ & 工 & Electric Co & & Total & & & 1.095 & 3.608 & & \\
\hline $\begin{array}{c}\text { Tolal } \\
\text { PENNSYLVANIA }\end{array}$ & & & 1.075 & 1,130 & & & $\begin{array}{l}\text { WIsconsin } \\
\text { Carllon }\end{array}$ & $\begin{array}{l}\text { Kewaunee Nuclear } \\
\text { Power Plant }\end{array}$ & FPl. & 503 & 535 & $\begin{array}{l}\text { Wisconsin Public } \\
\text { Senvice Corp }\end{array}$ & 7403 \\
\hline Berwick & $\begin{array}{l}\text { Susquehanna Steam } \\
\text { Electric Station. }\end{array}$ & $F P_{L}$ & 1,032 & 1,050 & $\begin{array}{l}\text { Pennsylvania } \\
\text { Power \& }\end{array}$ & 8209 & Two Creeks & $\begin{array}{l}\text { Poinl Beach Nuclear } \\
\text { Plant, Unit I }\end{array}$ & FPL & 485 & 497 & $\begin{array}{l}\text { Wisconsin Electric } \\
\text { Power } \mathrm{Co}\end{array}$ & 7011 \\
\hline Berwick & $\begin{array}{l}\text { Unil I } \\
\text { Susquehanna Steam }\end{array}$ & FPL & 1.032 & 1.050 & $\begin{array}{l}\text { Light Co } \\
\text { Pennsylvania }\end{array}$ & 8405 & Two Creeks & $\begin{array}{l}\text { Point Beach Nuclear } \\
\text { Plant. Unit? }\end{array}$ & FPL & 485 & 497 & $\begin{array}{l}\text { Wisconsin Eleciric } \\
\text { Power Co }\end{array}$ & 7205 \\
\hline & $\begin{array}{l}\text { Electric Station. } \\
\text { Unit } 2\end{array}$ & & & & $\begin{array}{l}\text { Power \& } \\
\text { Light Co }\end{array}$ & & Tolai & & & 1.473 & 1,529 & & \\
\hline Lancaslet & $\begin{array}{l}\text { Peach Botiom Atomic } \\
\text { Power Station. Unit? }\end{array}$ & FPL & 1.051 & 1.065 & $\begin{array}{l}\text { Philadelphia } \\
\text { Efectric Co }\end{array}$ & 7309 & FPL, Full. $F$ & ower License & & Construction & Delerred/Halles & & \\
\hline Lancaster & $\begin{array}{l}\text { Peach Bollom Atomic } \\
\text { Power Station, Unit } 3\end{array}$ & FPL & 1.035 & 1.065 & $\begin{array}{l}\text { Philadalphia. } \\
\text { Electric Co. }\end{array}$ & 7408 & UC, Under & Active Construclion & SDU & Sthut Down & Under Review & & \\
\hline Middlatown & $\begin{array}{l}\text { Three Mile Island } \\
\text { Nuclear Station, } \\
\text { Unit } 1\end{array}$ & FPL & 776 & 819 & $\begin{array}{l}\text { GPU Nuclear } \\
\text { Corp. }\end{array}$ & 7406 & & & & & & & \\
\hline Pollstown & $\begin{array}{l}\text { Limerick Generating } \\
\text { Station, Unit } 1\end{array}$ & FPL & 1,055 & 1,055 & $\begin{array}{l}\text { Philadelphia } \\
\text { Electric } \mathrm{Cn}_{n}\end{array}$ & 8412 & & & & & & & \\
\hline Poltstown & $\begin{array}{l}\text { Lumerick Generating } \\
\text { Station, Unt } 2\end{array}$ & FPL & 1.065 & 1,065 & $\begin{array}{l}\text { Philacelphia } \\
\text { Electric Co }\end{array}$ & 8912 & & & & & & & \\
\hline Shippingport & $\begin{array}{l}\text { Beaver Valley Power } \\
\text { Station. Unil ? }\end{array}$ & $\mathrm{FPL}$ & 810 & 835 & $\begin{array}{c}\text { Duquesne Light Co } \\
\text { Ohio Edison Co }\end{array}$ & 7605 & & & & & & & \\
\hline Shippingport & $\begin{array}{l}\text { Beaver Valley Power } \\
\text { Stalion, Unir ? }\end{array}$ & FPL & 852 & 836 & $\begin{array}{l}\text { Duquesne Light Co. } \\
\text { Ohio Edison } \mathrm{Co} \text {. }\end{array}$ & 8708 & & & & & & & \\
\hline Tolal & & & 8.708 & 8,840 & & & & & & & & & \\
\hline
\end{tabular}


DOE/OSTI-8200-R56

(DE93015065)

August 1993

Distribution Categories UC-500, UC-522, UC-523, UC-530, and SO820

ISSN: 0364-6866 CODEN: NABPDB

\section{Nuclear Reactors Built, Being Built, or Planned 1992}

Prepared for:

U.S. DEPARTMENT OF ENERGY

Director, Office of Nuclear Energy

Washington, D.C. 20585

Prepared by:

Office of Scientific and Technical Information 


\section{Preface}

Nuclear Reactors Built, Being Built, or Planned contains unclassified information about facilities built, being built, or planned in the United States for domestic use or export as of December 31, 1992. The Office of Scientific and Technical Information, U.S. Department of Energy, gathers this information annually from Washington headquarters and ficld offices of DOE; from the U.S. Nuclear Regulatory Commission (NRC); from the U.S. reactor manufacturers who are the principal nuclear contractors for foreign reactor locations; from U.S. and foreign embassies; and from foreign governmental nuclear departments.

The book consists of three divisions, as follows:

- a commercial reactor locator map and tables of the characteristic and statistical data that follow; a table of abbreviations;

- Lables of data for reactors operating, being built, or planned; and

- tables of data for reactors that have been shut down permanently or dismantled.

The reactors are subdivided into the following parts: Civilian, Production, Military, Export, and Critical Assembly. Export reactor refers to a reactor for which the principal nuclear contractor is an American company-working cither independently or in cooperation with a foreign company (Part IV). Critica! assembly refers to an assembly of fuel and moderator that requires an external source of neutrons to initiate and maintain fission. A critical assembly is used for experimental measurements (Part V).

Various classes of reactors within these parts are defined as follows:

Central-Station Electric Power Plant: A nuclear power facility designed and constructed for operation on a utility system (Part I, Section 1A).

Dual-Purpose Plant: A nuclear power facility designed, constructed, and operated for more than one primary purpose, for example, the production of nuclear materials and the generation of electricity or the use of reactor thermal energy for electrical gencration and process-heat applications including desalting (Part I, Section 1B).

Experimental Power Reactor: A facility designed, engineered, constructed, and operated to test the technical feasibility of a concept or to provide the technical basis for a similar type nuclear power plant in a larger size. Design flexibility permits changes to prove various aspects of reactor technology including fuel, components, and configurations. Power-conversion equipment may or may not be included as part of the facility (Part I, Section 2).

General Irradiation Test Reactor: A reactor having (1) a thermal power level exceeding $10 \mathrm{MW}$; (2) test loops or experimental facilities within, or in proximity to, the core; and (3) the use of nuclear radiation for testing the life or performance of reactor components as its major function (Part I, Section 3A; Part IV, Section 2A).

High-Power Research and Test Reactor: A reactor having a relatively high thermal power level (5MW or greater) but not classed as a general irradiation test reactor (Part I, Section 3B).

Safety-Research and Test Reactor: A reactor associated with a nuclear safety research or engineering-scale test program conducted for the purpose of developing basic design information or demonstrating safety characteristics of terrestrial and aerospace nuclear reactor systems (Part I, Section 3C).

General Research Reactor: A reactor-excluding that located at a university - whose nuclear radiations are used primarily as a research tool for basic or applied research and whose thermal power level is $10 \mathrm{MW}$ or less. It may include facilities for testing reactor materials (Part I, Section 3D; Part IV, Section 2B). Also included are Rescarch Reactors (Part III, Section 3B).

University Research and Teaching Reactor: A reactor located at a university and usually operated for the primary purpose of training in the operation and utilization of reactors and for instruction in reactor theory and performance (Part I, Section 3E; Part IV, Section 2C).

Reactors are further grouped according to status:

Reactors are listed as operable under the following circumstances:

1. Reactors regulated by the NRC

- when an operating license is issued.

- when a reactor is temporarily shut down because of technical reasons, modifications, or refueling.

2. Federal Government reactors

- when criticality is achieved.

- when a reactor is temporarily shut down for safety improvements.

3. Reactors for export

- when criticality is achieved.

Reactors are listed as being built under the following circumstances:

1. Reactors regulated by NRC

- when a construction permit is issued.

- when limited work authorization is issued.

2. Federal Government reactors

- when ground is broken.

- when components are ordered.

- when a construction contract is awarded. 
3. Reactors for export

- when an application for an export license is received by NRC.

- when reliable information is received relating to the fabrication of reactor components.

Reactors are listed as planned under the following circumstances:

1. Reactors regulated by NRC

- when a public announcement that includes the principal vendor supplier is made by the sponsoring organization.

- when an application for a construction permit is received by NRC.

2. Federal Government reactors

- when a public announcement is made by the agency involved.

- when the project is otherwise appropriately authorized.

3. Reactors for export

- when a public announcernent that includes principal contractor and reactor type is made.

- when NRC receives information that a U.S. reactor manufacturer is proceeding with preconstruction design and development on the basis of a letter of intent.

Reactors are considered to be shutdown or dismantled under the following circumstances:

1. Reactors regulated by NRC

- when the licensee has applied to the Commission for authority to surrender a license voluntarily and to dismantle the facility and dispose of its component parts. A reactor shut down because of technical problems, modifications, or refueling, continues to be listed as operable.

2. Federal Government reactors

- when the facility has ceased operation and the agency has declared officially that the agency does not intend to operate the reactor further. However, within this category, a few reactors are identified as being in standby mode, the condition in which documentary authorization exists to maintain the reactor for possible future operation.
3. Reactors for export

- when the plant is officially declared shut down by the owner and taken out of operation permanently.

Table 2 presents a statistical summary of reactors, other than critical assemblies, in each class and status. Shutdown and dismantled reactors are included since such facilities have made significant contributions to reactor technology.

The reac or tables have the following column headings:

- Location. The city and state or country where located originally. For a portabie facility or one that has been relocated, the most recent location is given.

- Principal nuclear contractor, operator, designer, shipbuilder. The abbreviations used in this column are spelled out in Table 3, which appears just before the reactor tables.

- Type. Entries in this column are based on coolant, moderator, and neutron energy.

- Power. MD capacity [MW(e)] is the maximum dependable capacity (net electrical output to grid) for plants having an operating history. Otherwise, it is the design capacity. Licensed power and authorized power are given where appropriate.

- Designation. The common name, abbreviation or acronym used for the facility. For the naval reactors, it is the hull number.

- Date columns. The initial criticality date, year of operation, and year of shutdown are given as appropriate.

Nuclear Reactors Built, Being Built, or Planned (DOE/ OSTI--8200-R56) is sponsored by the DOE Office of Nuclear Energy, LaRue E. Moxley, Program Officer.

The participation and assistance of many individuals, agencies, and companies in providing data and updating the entries in this revision are gratefully acknowledged. Comments and suggestions about this publication are welcome. To ensure that the wide range of information included in this publication will continue to be timely and accurate, please direct any information related to updating the items to William F. Simpson, Jr., Office of Scientific and Technical Information, Scientific and Technical Publications Branch, P.O. Box 62, Oak Ridge, TN 37831; (615) 576-1228. Questions of a technical nature should be addressed to Lamar Cason at the same address. 


\section{CONTENTS}

Preface

Map: Commercial Nuclear Power Reactors in the United States

Table 1. Commercial Nuclear Power Reactors in Operation in the United States

Table 2. Statistical Summary of Nuclear Reactors

ix

Table 3. Abbreviations of Contractors, Designers, Shipbuilders, and Facility Operators

\section{REACTORS AND FACILITIES}

\section{OPERABLE, BEING BUILT, OR PLANNED}

\section{Part I. Civilian Reactors (Domestic)}

1. Power Reactors

A. Central-Station Electric Power Plants

B. Dual-Purpose Plants (No reactors currently in this category)

C. Propulsion (Maritime) (No reactors currently in this category)

2. Experimental Power-Reactor Systems
A. Electric-Power Systems
B. Space Nuclear Auxiliary Power (SNAP)
C. Space Propulsion (No reactors currently in this category)

3. Test, Research, and University Reactors
A. General Irradiation Test
B. High-Power Research and Test
C. Safety-Research and Test
D. General Research
E. University Research and Teaching

Part II. Production Reactors

1. Materials Production

2. Process Development (No reactors currently in this category) 


\section{CONTENTS (Continued)}

Part III. Military Reactors

1. Defense Power-Reactor Applications

A. Remote Installations (No reactors currently in this category)

B. Propulsion (Naval)

2. Developmental Power

A. Electric-Power Experiments and Prototypes (No reactors currently in this category)

B. Propulsion Experiments and Prototypes 15

3. Test and Research

A. Test (No reactors currently in this category)

B. Research

Part IV. Export Reactors

1. Power Reactors

A. Central-Station Electric Power Plants

B. Propulsion (No reactors currently in this category)

2. Test, Research, and Teaching

A. General Irradiation Test

B. General Research

C. University Research and Teaching

Part V. Critical Assemblles

1. Civilian

2. Military

\section{REACTORS AND FACILITIES SHUTDOWN OR DISMANTLED}

\section{Part I. Civilian Reactors (Domestic)}

1. Power Reactors

A. Central-Station Electric Power Plants 25

B. Dual-Purpose Plants

C. Propulsion (Maritime)

2. Experimental Power-Reactor Systems

A. Electric-Power Systems

B. Space Nuclear Auxiliary Power (SNAP)

C. Space Propulsion

3. Test, Research, and University Reactors

A. General Irradiation Test

B. High-Power Research and Test

C. Safety-Research and Test

D. General Research

E. University Research and Teaching 


\section{CONTENTS (Continued)}

\section{Part II. Production Reactors}

1. Materials Production

2. Process Development

Part III. Military Reactors

$\begin{array}{ll}\text { 1. Defense Power-Reactor Applications } & 40\end{array}$

$\begin{array}{ll}\text { A. Remote Installations } & 40\end{array}$

B. Propulsion (Naval) 41

2. Developmental Power 43

A. Electric-Power Experiments and Prototypes 43

B. Propulsion Experiments and Prototypes 43

3. Test and Research 43
A. Test

B. Research

Part IV. Export Reactors

1. Power Reactors 44

A. Central-Station Electric Power Plants 44

B. Propulsion 44

2. Test, Research, and Teaching 45

A. General Irradiation Test (No reactors currently in this category)
B. General Research

$\begin{array}{ll}\text { C. University Research and Teaching } & 45\end{array}$

Part V. Critical Assemblies

1. Civilian

2. Military 


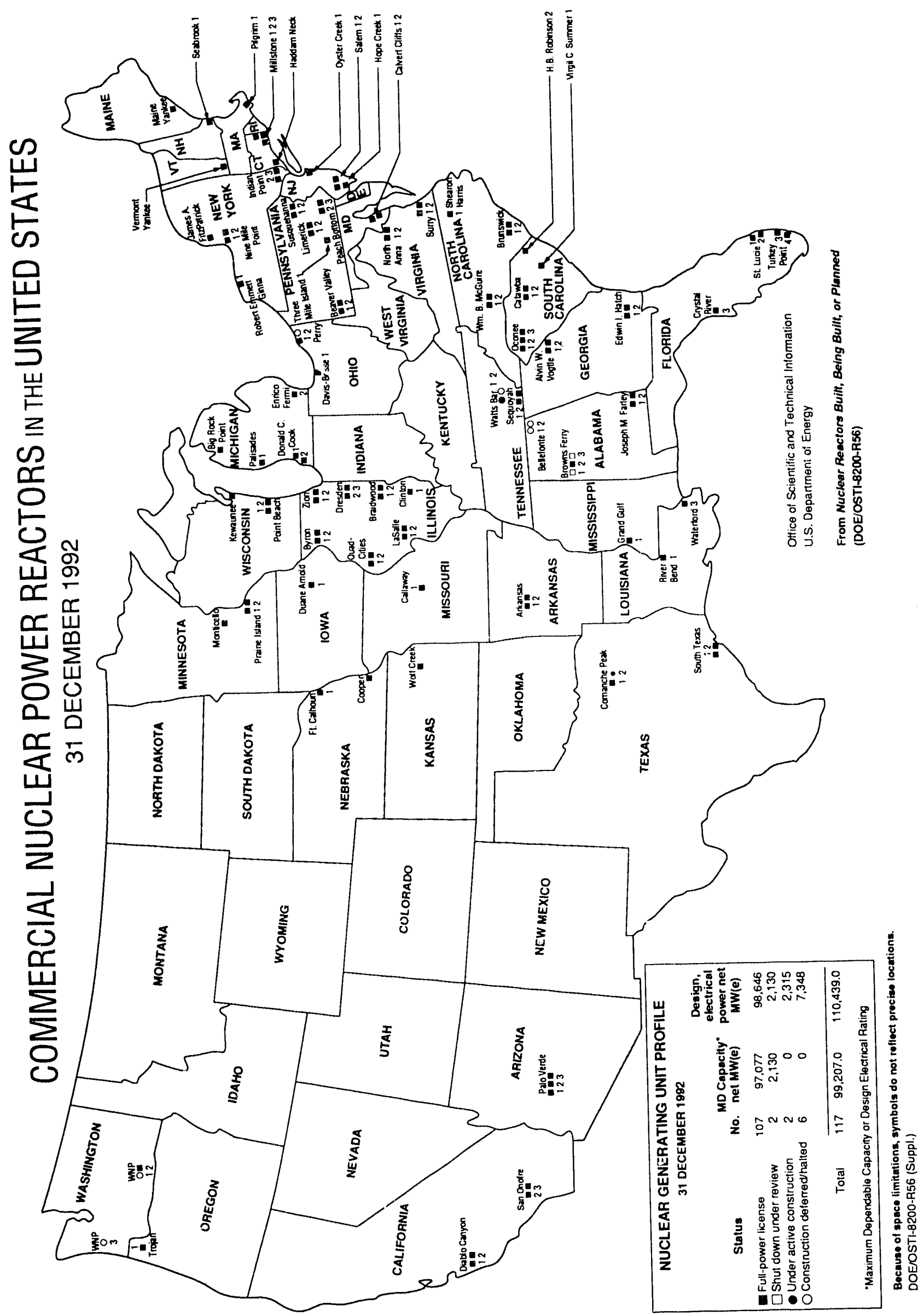

viii 
TABLE 1

COMMERCIAL NUCLEAR POWER REACTORS IN THE UNITED STATES AS OF 31 DEC 1992

STTE

PLANT NAME

ALABAMA

Decatur

Decatur

Decatur

Dothan

Dothan

Scottsboro

Scottsboro

Total

ARIZONA

Wintersburg

Wintersburg

Wintersburg

Total

\section{ARKANSAS}

Russellville

Russellville

Total

CALIFORNIA

Diablo Canyon

Diablo Canyon

San Clemente

San Clemente

Total

CONNECTICUT

Haddam Neck

Waterford

Waterford

Waterford

Station, Unit 1

Station, Unit 2

Station, Unit 3 Unit 1 Unit 2 Station, Unit 1 Station, IJnit 2 Station, Unit 3 Plant, Unit 1

Plant, Unit 2

Station, Unit 2 Station, Unit 3

Haddam Neck Plant

Millstone Nuclear Power

Station, Unit 1

Millstone Nuclear Power

Station, Unit 2

Millstone Nuclear Power

Station, Unit 3

\section{CAPACITY, POWER NET}

STATUS NET MW(e) MW(e)

LICENSEE

STARTUP

Browns Ferry Nuclear Power

Browns Ferry Nuclear Power

Browns Ferry Nuclear Power

Joseph M. Farley Nuclear Plant,

Joseph M. Farley Nuclear Plant,

Bellefonte Nuclear Plant, Unit 1

Bellefonte Nuclear Plant, Unit 2

$\begin{array}{lrr}\text { SDUR } & 1,065 & 1,065 \\ \text { FPL } & 1,065 & 1,065 \\ \text { SDUR } & 1,065 & 1,065 \\ \text { FPL } & 825 & 829 \\ \text { FPL } & 824 & 829 \\ \text { CDH } & & 1,235 \\ \text { CDH } & & 1,235 \\ & 4,844 & 7,323\end{array}$

FPL

1,221

1,270

Palo Verde Nuclear Generating

Palo Verde Nuclear Generating

Palo Verde Nuclear Generating

FPL

$1,22 !$

1,270

FPL

1,304

1,270

3,746

3,810

Arkansas Nuclear One, Unit 1 Arkansas Nuclear One, Unit 2

FlL

\begin{tabular}{rr}
836 & 850 \\
858 & 912 \\
\hline 1,694 & 1,762
\end{tabular}

Diablo Canyon Nuclear Power

Diablo Canyon Nuclear Power

San Onofre Nuclear Generating

San Onofre Nuclear Generating

FPL

1,073

1,086

FPL

1,079

1,119

FPL

1,070

1,070

FPL

1,080

1,080

4,302

4,355

FPL $\quad 569$

582
Tennessee Valley Authority

Tennessee Valley Authority

Tennessee Valley Authority

Southem Nuclear Operating

Co.

Southem Nuclear Operating

Co.

Tennessee Valley Authority

Tennessee Valley Authority

7308

7407

7608

7708

8105

Indef.

Indef.

Arizona Public Service Co.

8505

Arizona Public Service Co.

8604

Arizona Public Service Co.

8710

Entergy Operations Inc.

7408

Entergy Operations Inc.

7812

Pacific Gas \& Electric Co.

8404

Pacific Gas \& Electric Co.

8508

Southem California Edison

8207

Southem California Edison

8308

Connecticut Yankee Atomic

6707

Power Co.

Northeast Nuclear Energy Co.

7010

Northeast Nuclear Energy Co.

7510

Northeast Nuclear Energy Co.

8601 $\begin{array}{lll}\text { FPL } & 654\end{array}$

$\begin{array}{lll}\text { FPL } & 857\end{array}$

FPL $\quad 1,142 \quad 1,154$

$1,142 \quad 1,154$

3,222

3,266 
TABLE 1 (Continued)

SITE

PLANT NAME

CAPACITY

POWER NET

FLORIDA

Florida City

Florida City

Fort Pierce

Fort Pierce

Red Level

Turkey Point Plant, Unit 3

Turkey Point Plant, Unit 4

St. Lucie Plant, Unit 1

St. Lucie Plant, Unit 2

Crystal River Nuclear Plant, Unit 3

STATUS

NET MW(e)

MW(e)

LICENSEE

STARTUP

Total

\section{GEORGIA}

Baxley

Baxley

Waynesboro

Waynesboro

Edwin I. Hatch Nuclear Plant, Unit 1

Edwin I. Hatch Nuclear Plant, Unit 2

Alvin W. Vog̣tle Nuclear Plant, Unit 1

Alvin W. Vogtle Nuclear Plant, Unit 2

\section{ILLINOIS}

Braidwood

Braidwood

Byron

Byron

Clinton

Cordova

Cordova

Morris

Morris

Seneca

Seneca

Zion

Zion

Total

Braidwood Station, Unit 1

Braidwood Station, Unit 2

Byron Station, Unit 1

Byron Station, Unit 2

Clinton Power Station, Unit 1

Quad-Cities Station, Unit 1

Quad-Cities Station, Unit 2

Dresden Nuclear Power Station, Unit 2

Dresden Nuclear Power Station, Unit 3

La Salle County Station, Unit 1

La Salle County Station, Unit 2

Zion Nuclear Plant, Unit 1

Zion Nuclear Plant, Unit 2

IOWA

Palo

Duane Amold Energy Center, Unit 1

Total

KANSAS

Burlington

Total

Wolf Cres! Generating Station

\section{LOUISIANA}

St. Francisville Taft

River Bend Station, Unit 1

Waterford Generating Station, Unit 3

Total

\begin{tabular}{lll} 
FPL & 666 & 693 \\
FPL & 666 & 693 \\
FPL & 839 & 830 \\
FPL & 839 & 830 \\
FPL & 821 & 825 \\
\hline
\end{tabular}

3,831

3,871

$\begin{array}{lrr}\text { FPL } & 750 & 776 \\ \text { FPL } & 761 & 784 \\ \text { FPL } & 1,101 & 1,101 \\ \text { FPL } & 1,101 & 1,101 \\ & 3,713 & 3,762\end{array}$

\begin{tabular}{lrr} 
FPL & 1,120 & 1,120 \\
FPL & 1,120 & 1,120 \\
FPL & 1,129 & 1,120 \\
FPL & 1,129 & 1,120 \\
FPL & 950 & 933 \\
FPL & 769 & 789 \\
FPL & 769 & 789 \\
FPL & 772 & 794 \\
FPL & 773 & 794 \\
FPL & 1,036 & 1,078 \\
FPL & 1,036 & 1,078 \\
FPL & 1,040 & 1,040 \\
FPL & 1,040 & 1,040 \\
& & \\
\cline { 2 - 3 } & 12,683 & 12,815
\end{tabular}

IFPL

$$
515
$$

538

FPL

\begin{tabular}{ll}
1,128 & 1,170 \\
\hline 1,128 & 1,170
\end{tabular}

Florida Power \& Light Co.

Florida Power \& Light Co.

Florida Power \& Light Co.

Florida Power Corp.
93 Florida Power \& Light Co.

7210

7306

7604

8306

7701

7409

7807

8703

8903

Georgia Power Co.

8705

$88 C 3$

8502

8701

8702

7110

7204

7001

7101

8206

8403

7306

7312

Commonwealth Edison Co.

7403

lowa Electric Light \& Power Co.

Kansas Gas \& Electric Co.

8505

FPL $\quad 936 \quad 936$

$\begin{array}{lll}\text { FPL } & 1,075 & 1,104\end{array}$

2,011

2,040
Gulf States Utilities Co.

Entergy Operations Inc. 
TABLE 1 (Continued)

SITE

MAINE

Wiscasset

\section{MARYLAND}

Lusby

Lusby

Total

MASSACHUSETTS

Plymouth

Pilgrim Nuclear Power Station,

Total

MICHIGAN

Big Rock Point

Bridgman

Bridgman

Newpon

South Haven

Total

\section{MINNESOTA}

Monticello

Red Wing

Red Wing

Total

MISSISSIPPI

Pon Gibson

Total

MISSOURI

Fulton

Total

\section{NEBRASKA}

Brownville

Fort Calhoun

Total Plant

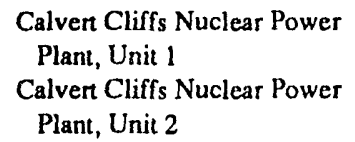

Big Rock Point Nuclear Plant Donald C. Cook Nuclear Power Plant, Unit 1

Donald C. Cook Nuclear Power Plant, Unit 2

Enrico Ferni Atomic Power

Plant, Unit 2

Palisades Nuclear Plant, Unit 1

Monticello Nuclear Generating Plant Plant, Unit 1 Plant, Unit 2 Unit 1

Callaway Plant, Unit 1

Cooper Nuclear Station Fort Calhoun Station, Unit
Maine Yankee Atomic Power FPL

Prairie Island Nuclear Generating

Prairie Island Nuclear Generating

Grand Gulf Nuclear Station,

FPL

\begin{tabular}{rrr} 
FPL & 69 & 72 \\
FPL & 1,020 & 1,030 \\
FPL & 1,060 & 1,100 \\
FPL & 1,093 & 1,093 \\
FPL & 730 & 805 \\
\cline { 2 - 3 } & 3,972 & 4,100
\end{tabular}

FPL

FPL

FPL

FPL

$$
825
$$

845

845

FPL 825

690

$1,650 \quad 1,690$

FPL

$$
670
$$

655

670

3,972

4,100

PPL $\quad 536$

$$
503
$$

545

530

$$
500
$$

530

1,539

1,605

1,142

1,250

1,142

1,250
FPL

$\begin{array}{ll}1,120 & 1,171 \\ 1,120 & 1,171\end{array}$

FPL.

FPL

\begin{tabular}{ll}
764 & 778 \\
478 & 478 \\
\hline 1,242 & 1,256
\end{tabular}

LICENSEE

STARTUP

7210 Power Co.

Baltimore Gas \& Electric Co.

7410

Baltimore Gas \& Electric Co.

7611

Boston Edison Co.

7206

6209

7501

7803

8506

7105

Consumers Power Co.

7012

7312

7412

Northern States Power Co.

8208

Entergy Operations Inc.

8410

Union Electric Co.

$\begin{array}{ll}\text { Nebraska Public Power District } & 7402 \\ \text { Omaha Public Power District } & 7308\end{array}$ 
TABLE 1 (Continued)

SITE

PLANT NAME

NEW HAMPSHIRE

Seabrook

Total

\section{NEW JERSEY}

Salem

Salem

Salem

Toms River

Total

\section{NEW YORK}

Buchanan

Buchanan

Ontario

Scriba

Scriba

Scriba

$$
\text { Total }
$$

\section{NORTH CAROLINA}

Bonsal

Cowans Ford Dam

Cowans Ford Dam

Southport

Southport

Total

\section{OHIO}

Oak Harbor

Perry

Perry Station, Unit 1

Station, Unit 1

Station, Unit 2

Plant, Unit 1

Power Plant, Unit 1

Power Plant

Nine Mile Point Nuclear

Station, Unit 1

Nine Mile Point Nuclear

Station, Unit 2 Plant, Unit 1 Station, Unit 1 Station, Unit 2 Unit 1 Unit 2
Seabrook Nuclear Station, Unit 1

FPL

\begin{tabular}{ll}
1,198 & 1,198 \\
\hline 1,198 & 1,198
\end{tabular}

Hope Creek Nuclear Generating

Salem Nuclear Generating

Salem Nuclear Gencrating

Oyster Creek Nuclear Power

\begin{tabular}{lrr} 
FPL & 1,067 & 1,067 \\
FPL & 1,106 & 1,115 \\
FPL & 1,106 & 1,115 \\
FPL & 620 & 650 \\
\hline
\end{tabular}

3,899

3,947

Indian Point Station, Unit 2

Indian Point Station, Unit 3

Roben Emmell Ginna Nuclear

James A. FitzPatrick Nuclear

FPL

$$
849
$$

873

FPL $\quad 1,000$

FPL $\quad 470$

965

FPL $\quad 794$

470

FPL

610

816

FPL

1,080

620

1,080

$4,803 \quad 4,824$

Shearon Harris Nuclear Power

William B. McGuire Nuclear

William B. McGuire Nuclear

Brunswick Stcam Electric Plant,

Brunswick Steam Electric Plant,

FPL

$$
915
$$

FPL

$$
1,150
$$

FPL

1,150

FPL

790

790

FPL

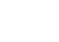

4,795

FPL

860

FPL

1,205

1,205

$\mathrm{CDH}$

Total
Perry Nuclear Power Plant, Unit 2

Davis-Besse Nuclear Power Station, Unit 1 Perry Nuclear Power Plant,
LICENSEE

STARTUP

Public Service Co. of New Hampshire

8906

Public Service Electric \&

8606

Gas Co.

Public Service Electric \& $\quad 7612$

Gas Co.

Public Service Electric \& $\quad 8008$

\begin{tabular}{|c|c|}
\hline $\begin{array}{l}\text { Consolidated Edison Co. of } \\
\text { New York, Inc. }\end{array}$ & 7305 \\
\hline New York Power Authority & 7604 \\
\hline Rochester Gas \& Electric Corp. & 6911 \\
\hline New York Power Authority & 7411 \\
\hline Niagara Mohawk Power Corp. & 6909 \\
\hline Viagara Mo & \\
\hline
\end{tabular}

Gas Co.

GPU Nuclear Corp.

6905

Carolina Power \& Light Co.

8701

Duke Power Co.

8108

Duke Power Co.

8305

Carolina Power \& Light Co.

7610

Carolina Power \& Light Co.

7503

Toledo Edison Co.

7708

Cleveland Electric

Illuminating $\mathrm{Co}$.

Cleveland Electric

Illuminating $\mathrm{Co}$.

8606

Indef. 
TABLE 1 (Continued)

SITE

PLANT NAME

Trojan Nuclear Plant, Unit

Total

PENNSYL VANIA

Berwick

Berwick

Lancaster

Lancaster

Middletown

Pottstown

Pottstown

Shippingport

Shippingport

Total

SOUTH CAROLINA

Hartsville

Jenkinsville

Lake Wylie

Lake Wylie

Seneca

Seneca

Seneca

Total

\section{TENNESSEE}

Daisy

Daisy

Spring City

Spring Ciiy

Total

TEXAS

Bay City

Bay City

Glen Rose

Glen Rose

Station, Unit 1

Station, Unit 2

Station, Unit 2

Station, Unit 3

Three Mile Island Nuclear

Station, Unit 1

Unit 1

Unit 2

Unit 1 Unit 2 Virgil C. Summer Nuclear

Station, Unit 1

South Texas Project, Unit 1

South Texas Project, Unit 2

Station, Unit 1
Susquehanna Steam Electric

Susquehanna Stcam Electric

Peach Bottom Atomic Power

Peach Bottom Atomic Power

Limerick Generating Station,

Limerick Generating Station,

Beaver Valley Power Station.

Beaver Valley Power Station,

H.B. Robinson Plant, Unit 2

Catawba Nuclear Station, Unit I

Catawba Nuclear Station, Unit 2

Oconec Nuclear Station, Unit 1

Oconce Nuclear Station, Unit 2

Oconce Nuclear Station, Unit 3

Sequoyah Nuclear Plant, Unit 1 Sequoyah Nuclear Plant, Unit 2

Watts Bar Nuclear Plant, Unit 1

Watts Bar Nuclear Plant, Unit 2

FPL

FPL

UC

C.DH

FPI

FPL

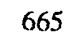

885

6,420

1,148

1,148

2,296

Comanche Peak Steam Electric

Comanche Peak Steam Electric

Station, Unit 2
TFIL

FPL

FPI.

UC

UC
700

Carolina Power \& Light Co.

7009

South Carolina Electric \&

Gas Co.

Duke Power Co.

Duke Power Co.

Dukc Power Co.

Duke Power Co.

Duke Power Co.

6,551

6,551

1,148

1,148

1,165

1,165

4,626
Tennessec Valley Authority

Tennessec Valley Authority

Tennessec Valley Authority

Tennessee Valley Authority
STARTUP

7512

8209

8405

7309

7408

7406

8412

8912

7605

8708

8210

8501

8605

7304

7311

7409

8007

8111

9312

Indef.

Houston Lighting \& Power Co. $\quad 8803$

Ilouston Lighting \& Power Co. $\quad 8902$

Texas Utilities Generating Co. $\quad 9004$

1,150 Texas Utilities Generating Co. $\quad 9212$ 
TABLE 1 (Continued)

SITE

Plant Name Vermont Yankee Nuclear Power
Station

Total

VIRGINIA

Gravel Neck

Gravel Neck

Mineral

Mineral

Total

WASHINGTON

Richland

Richland

Satsop

Total

\section{WISCONSIN}

Carlion

Two Creeks

Two Creeks

Total

U.S. Total

Surry Power Station, Unit 1

Surry Power Station, Unit 2

Washington Nuclear Project,

Unit 1

Washington Nuclear Project,

Unit 2

Washington Nuclear Project,

Unit 3

Kewaunee Nuclear Power Plant Point Beach Nuclear Plant,

Unit 1

Point Beach Nuclear Plant, Unit 2
CAPACITY, POWER NET

STATUS NET MW(e) MW(c)

$504 \quad 514 \quad$ Vermont Yankee Nuclear

Power Corp.
North Anna Power Station, Unit 1

North Anna Power Station, Unit 2

FPL

FPL

IPL

$504 \quad 514$

781

781
915

915

3,392

CDI1

FPL

1,095

1,266

$\mathrm{CDH}$

1,100

1,242

1,095

3,608

\begin{tabular}{lrr} 
& 1,095 & 3,608 \\
& & \\
FPL & 503 & 535 \\
FPL & 485 & 497 \\
FPL & 485 & 497 \\
& & \\
\cline { 2 - 3 } & 1,473 & 1,529 \\
\hline 99,207 & 110,439
\end{tabular}

CDH, Construction Deferred/Halted SDUR, Shut Down Under Review

FPL, Full-Power License

UC, Under Active Construction
STARTUP

7203

Virginia Electric \& Power Co. $\quad 7207$

Virginia Electric \& Power Co. $\quad 7303$

Virginia Electric \& Power Co. 7804

Virginia Electric \& Power Co. $\quad 8006$

Washirg.un Public Power Indef.

Supply System

Washington Public Power $\quad 8401$

Surply System

Washington Public Power Indef. Supply System

Wisconsin Public Service Corp. $\quad 7403$

Wisconsin Electric Power Co. $\quad 7011$

Wisconsin Electric Power Co. 7205 


\section{TABLE 2}

\section{STATISTICAL SUMMARY OF NUCLEAR REACTORS AS OF DECEMBER 1992}

Being
Operable Puilt Panned Shutdown Totals

\section{U.S. REACTORS}

CIVILIAN REACIORS (HOMESTIC)

Power Reactors

Central-Station Electric Power Plants

Dual-Purpose Plants

Propulsion (Maritime)

Experimental Power-Reactor Systems

Electric-Power Systems

Space Nuclear Auxiliary Power (SNAP)

Space Propulsion (Rover)

Test, Research, and University Reactors

General Irradiation Test

High-Power Research and Test

Safety-Researci and Test

General Research

University Research and Tcaching

PRODUC'TION REAC'TORS

Materials Production

rocess Develupment

MILITARY REACTORS

Defense Power-Reactor Applications

Remote Installations

Propulsion (Naval)

109

Elopmental Power

Electrit--Power Experiments and Prototypes

Propulsion Experiments and Prototypes

Test and Research

Test.

Rescarch

\section{EXPORT REAC'TORG}

POWER REACTORS

Central-Station Electric Power Plants

TEST, RESEARCH, AND TEACHING

General Irradiation T 'st

General Research

Unlversity Research and Teaching

Totals

$\begin{array}{r}7 \\ 27 \\ 19 \\ \hline 417\end{array}$

8

136

1

2

5

1

34

4

11

4

$$
5
$$

70

$\begin{array}{rr}19 & 136 \\ 1 & 1 \\ 1 & 1 \\ 23 & \\ 9 & 24 \\ 21 & 10\end{array}$

$\begin{array}{ll}7 & 12\end{array}$

$10 \quad 11$

$55-71$

$31 \quad 66$

$\begin{array}{rr}9 & 13 \\ 5 & 5\end{array}$

$\begin{array}{rr}6 & 6 \\ 59 & 211\end{array}$

$3 \quad 3$

$3 \quad 3$

$6 \quad 10$

\begin{tabular}{rrrr}
1 & 1 & 10 & 39 \\
-39 & - & 6 & 25 \\
\hline & 8 & 304 &
\end{tabular}

57


TABLE 3

\section{ABBREVIATIONS OF CONTRACTORS, DESIGNERS, SHIPBUILDERS, AND FACILITY OPERATORS}

The definitions of the following abbreviations that have been used in this volume contain references to current and historical corporate and government structure.

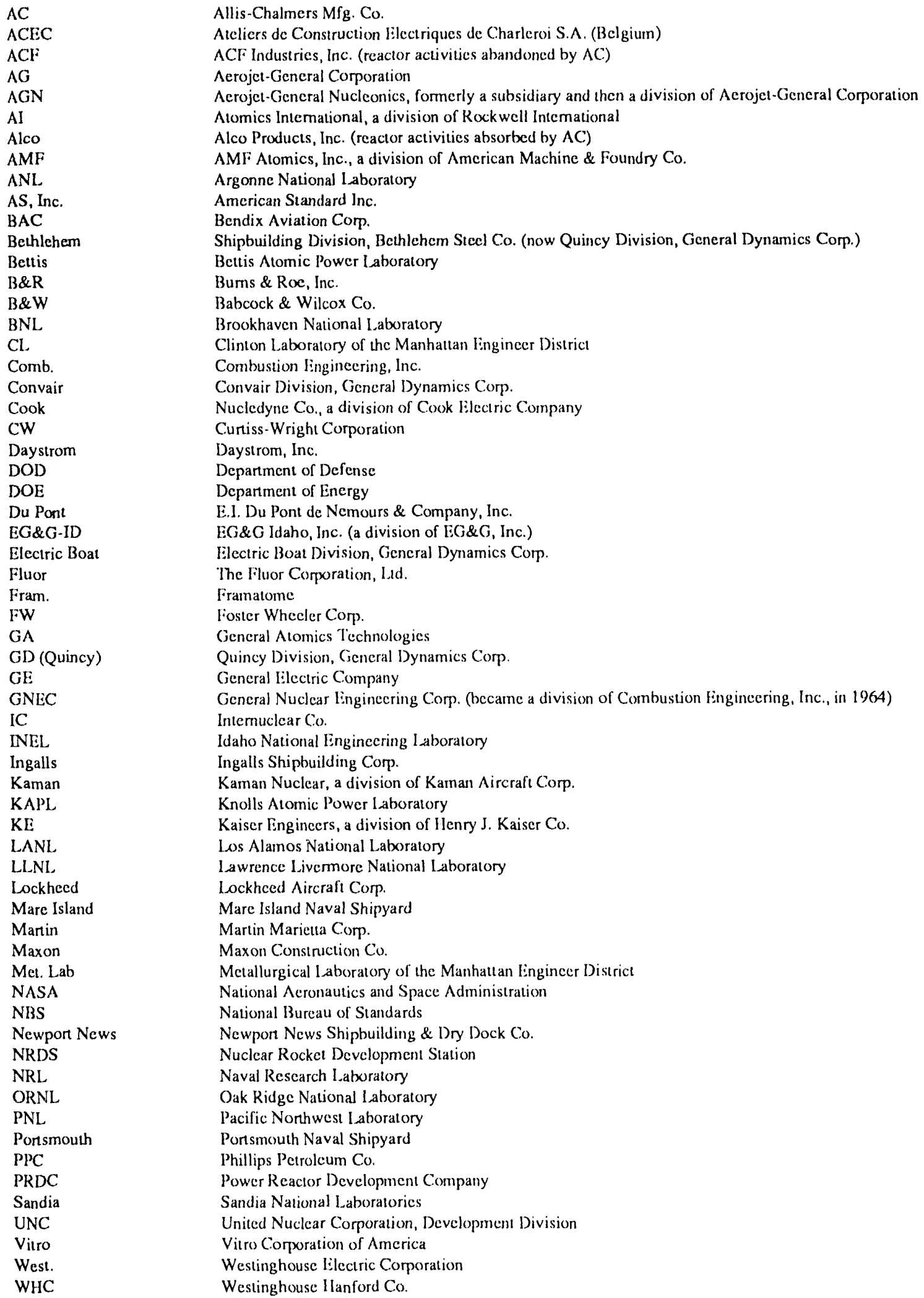

$A C$

ACEC

$\mathrm{ACF}$

$\mathrm{AG}$

AGN

AI

Alco

AMF

ANL

AS, Inc.

BAC

Bethlehem

Bettis

$B \& R$

B\&W

BNL

$\mathrm{CL}$

Comb.

Convair

Cook

CW

Daystrom

DOD

DOE

Du Pont

EG\&G-ID

Electric Boat

Fluor

Fram.

FW

GA

GD (Quincy)

$G E$

GNEC

IC

INEL

Ingalls

Kaman

KAPL

$\mathrm{KE}$

LANL

LLNL,

Lockheed

Mare Island

Martin

Maxon

Met. Lab

NASA

NBS

Newport News

NRDS

NRL

ORNL

PNL

Portsmouth

PPC

PRDC

Sandia

UNC

Vitro

West.

WHC

Allis-Chalmers Mfg. Co.

Ateliers de Construction Electriques de Charleroi S.A. (Belgium)

$\mathrm{ACF}$ Industries, Inc. (reactor activitics abandoned by $\mathrm{AC}$ )

Aerojet-General Corporation

Aerojet-General Nucleonics, formerly a subsidiary and then a division of Aerojet-General Corporation

Atomics International, a division of Rockwell Intemational

Alco Products, Inc. (reactor activities absorbed by $\mathrm{AC}$ )

AMF Alomics, Inc., a division of American Machine \& Foundry Co.

Argonne National Laboratory

American Standard Inc.

Bendix Aviation Corp.

Shipbuilding Division, Bethlehem Steel Co. (now Quincy Division, General Dynamics Corp.)

Bettis Atomic Power Laboratory

Bums \& Roc, Inc.

Babcock \& Wilcox Co.

Brookhaven National Laboratory

Clinton Laboratory of the Manhattan Engineer District

Combustion Fingineering, Inc.

Convair Division, General Dynamics Corp.

Nucledyne Co., a division of Cook Electric Company

Curtiss-Wright Corporation

Daystrom, Inc.

Department of Defense

Department of Energy

E.1. Du Pont de Nemours \& Company, Inc.

EG\&G Idaho, Inc. (a division of EG\&G, Inc.)

Electric Boat Division, General Dynamics Corp.

The Fluor Corporation, Lid.

Framatome

Foster Whecler Corp.

General Atomics Technologies

Quincy Division, General Dynamics Corp.

General Electric Company

General Nuclear Enginecring Corp. (became a division of Combustion Engineering, Inc., in 1964)

Internuclear Co.

Idaho National Enginecring I aboratory

Ingalls Shipbuilding Corp.

Kaman Nuclear, a division of Kaman Aircraft Corp.

Knolls Atomic Power Laboratory

Kaiser Engineers, a division of Henry J. Kaiser Co.

Los Alamos National Laboratory

Lawrence Livermore National Laboratory

Lockhced Aircrafi Corp.

Mare Island Naval Shipyard

Martin Marietta Corp.

Maxon Construction Co.

Metallurgical Laboratory of the Manhattan Engineer District

National Acronautics and Space Administration

National Bureau of Standards

Newpon News Shipbuilding \& Dry Dock Co.

Nuclear Rocket Development Station

Naval Rescarch Laboratory

Oak Ridge National Laboratory

Pacific Northwest Laboratory

Portsmouth Naval Shipyard

Phillips Petrolcum Co.

Power Reactor Development Company

Sandia National Laboratorics

United Nuclear Corporation, Developmem Division

Vitro Corporation of America

Westinghouse Electric Corporation

Westinghouse llanford Co. 


\section{REACTORS AND FACILITIES OPERABLE, BEING BUILT, OR PLANNED}




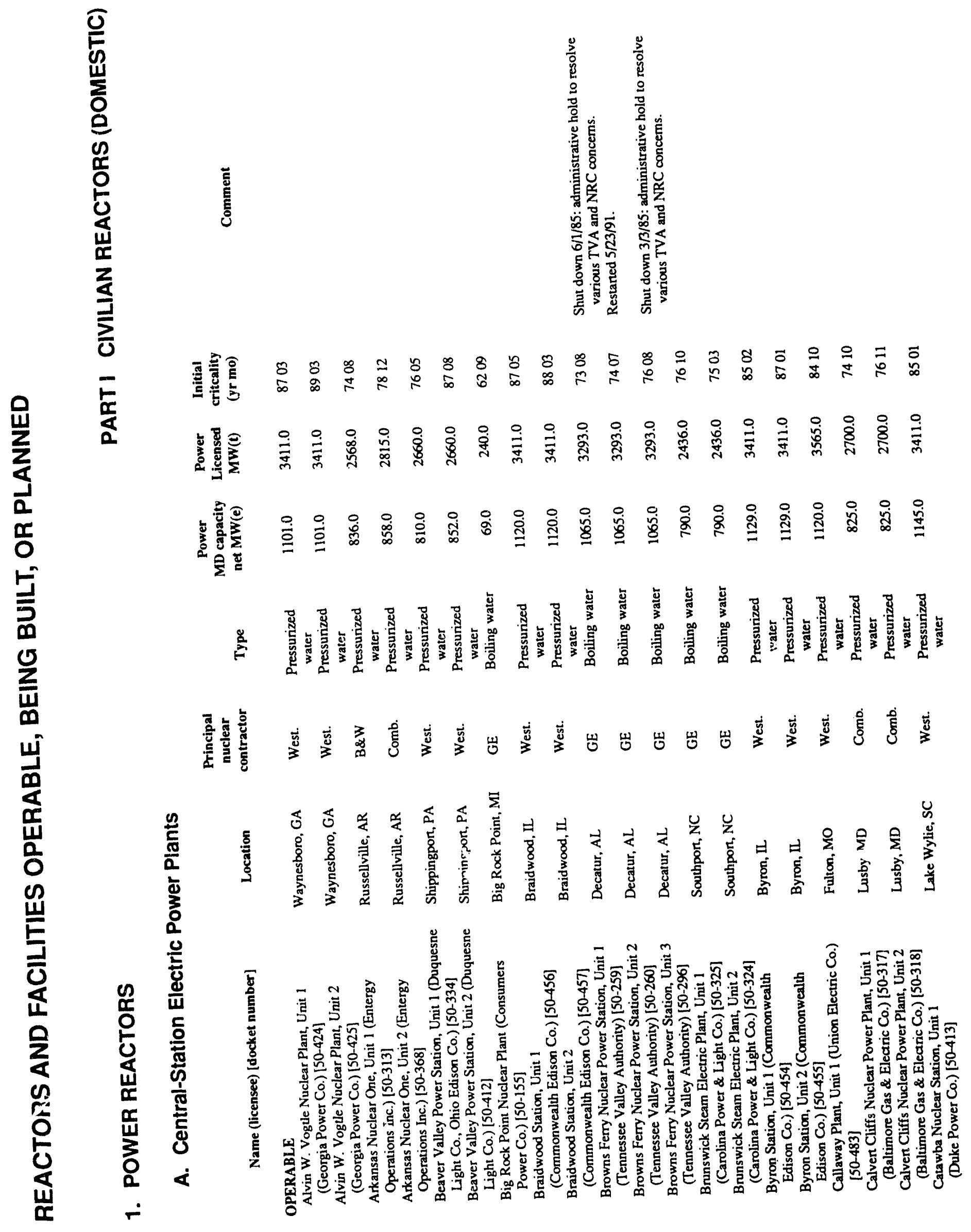




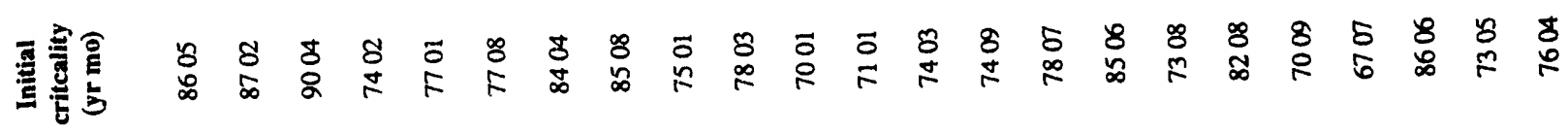

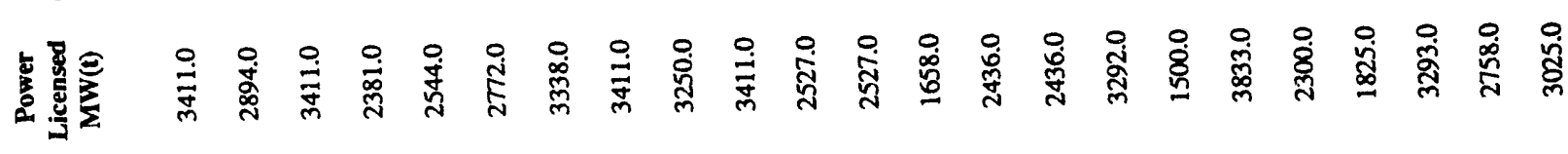

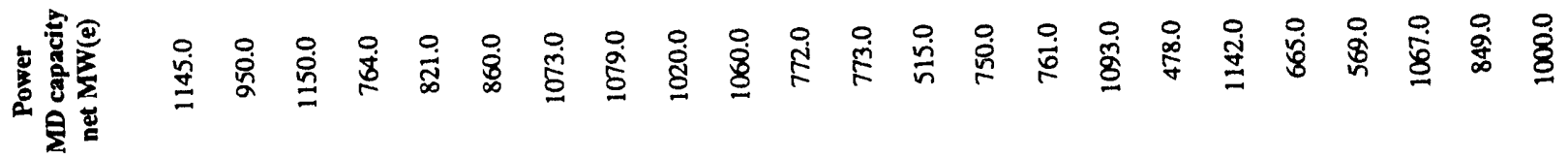

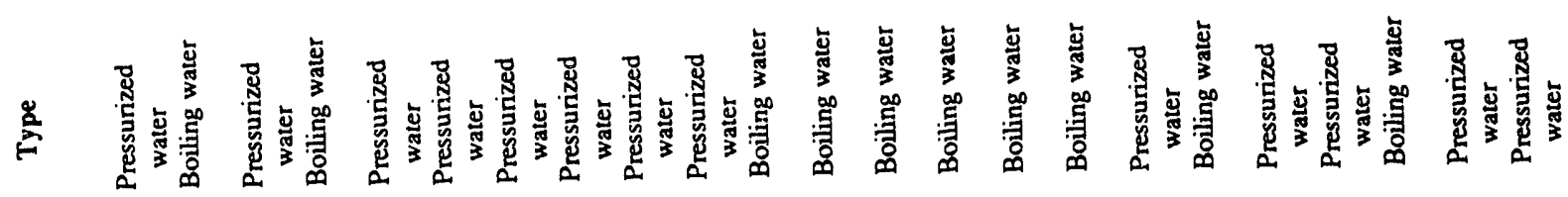

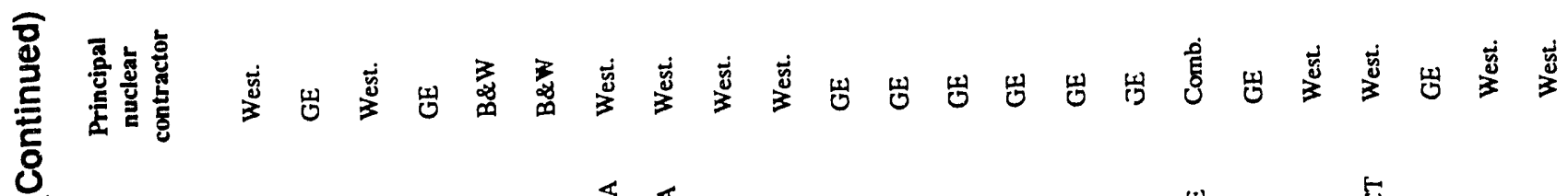

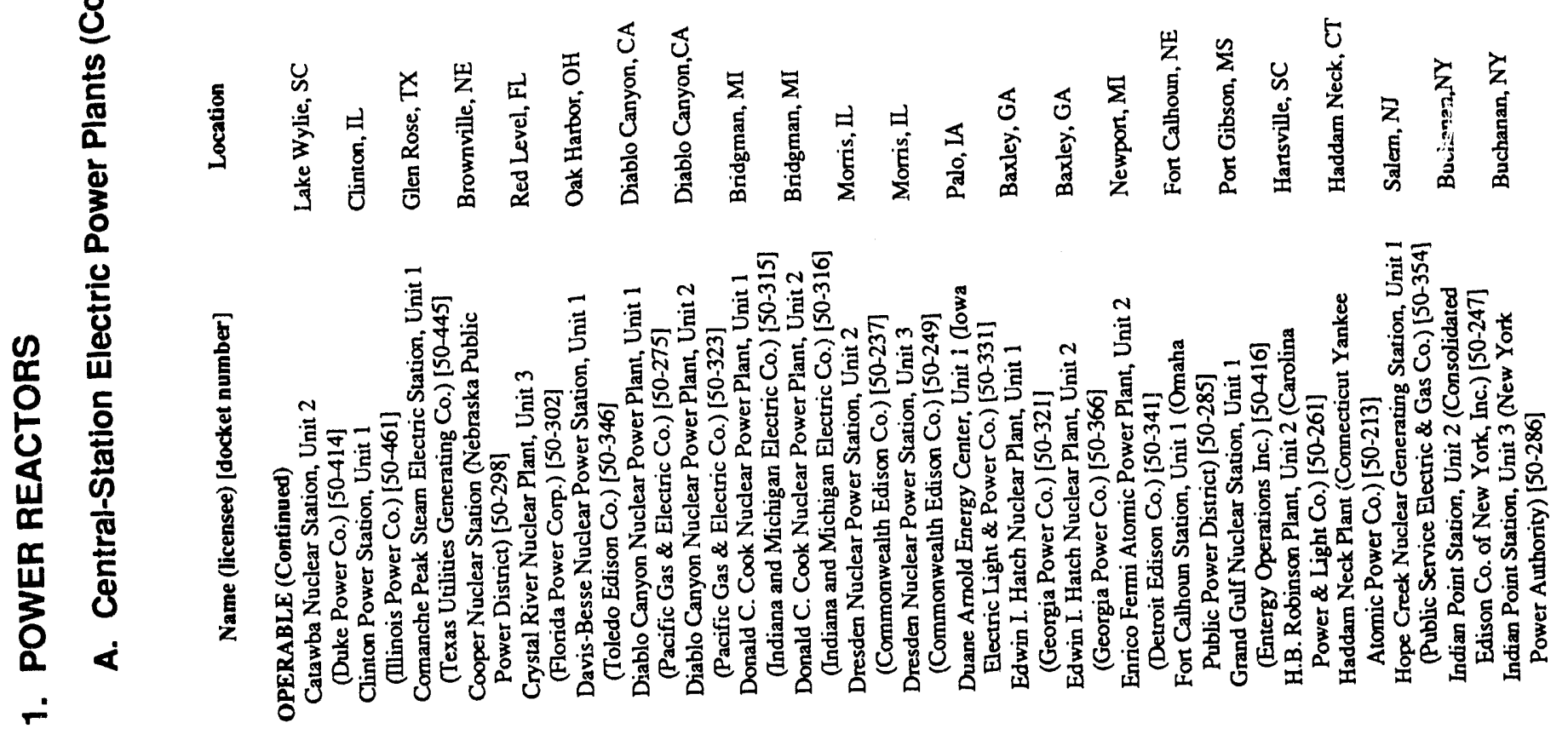




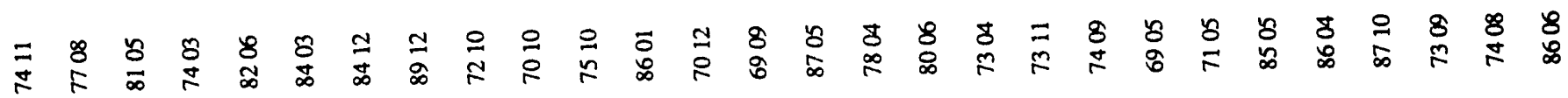

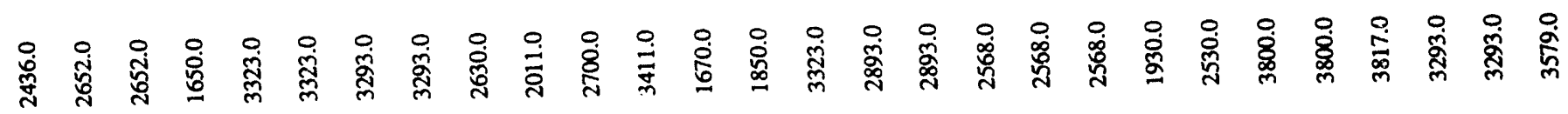

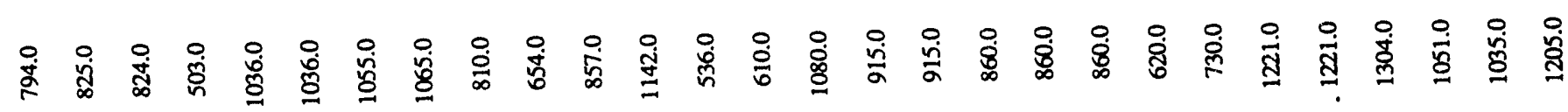
HWHHWWHWHMHWH

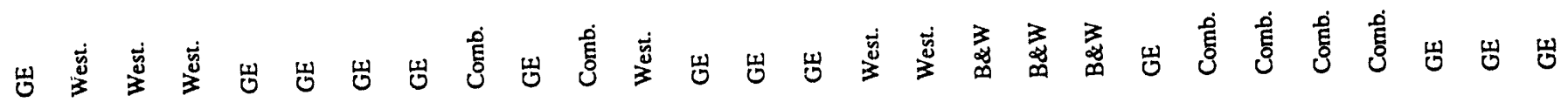

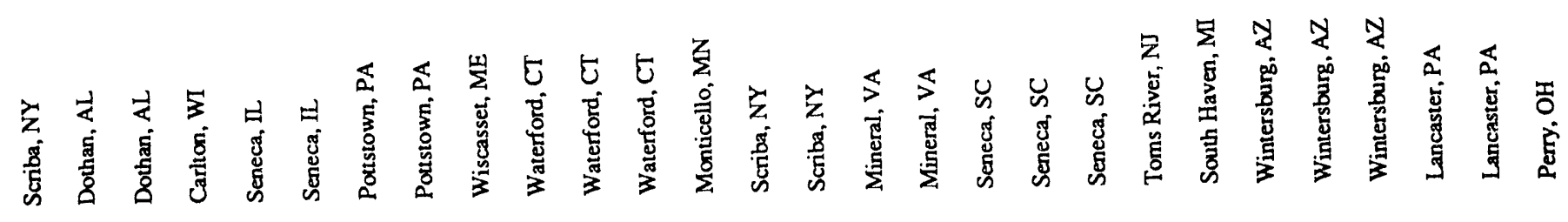

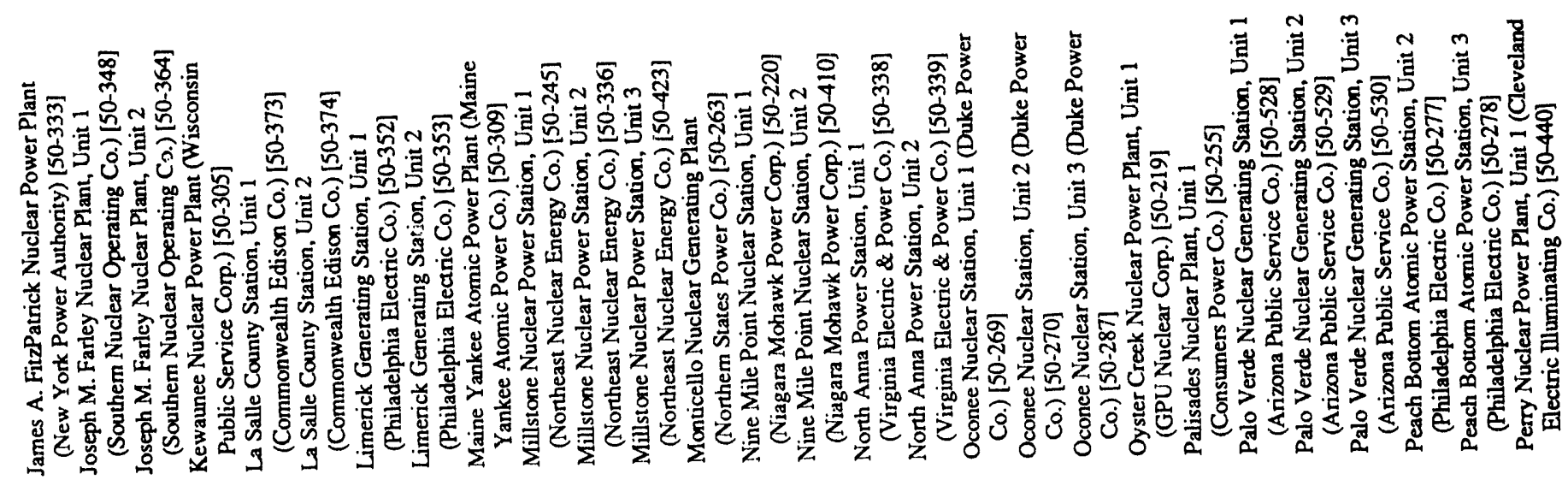




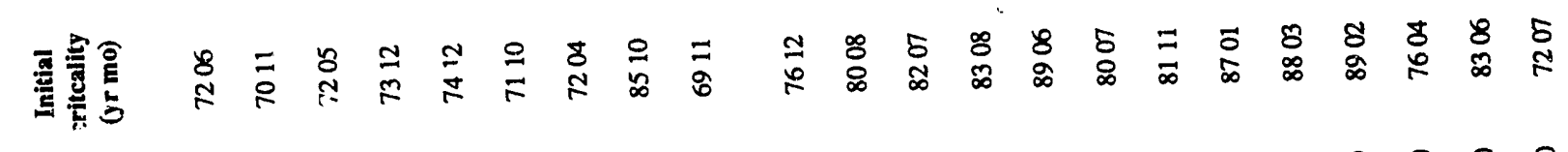

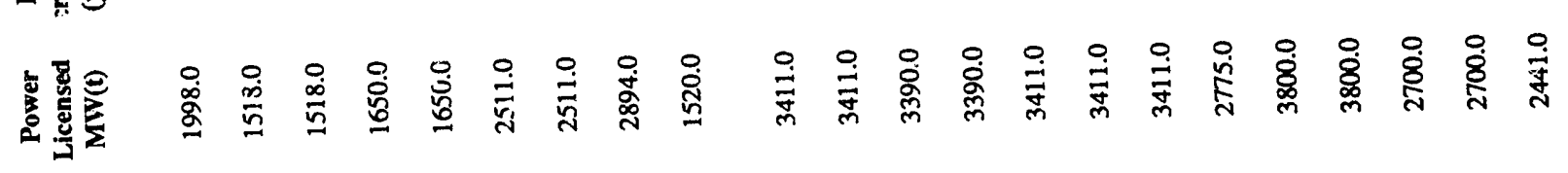

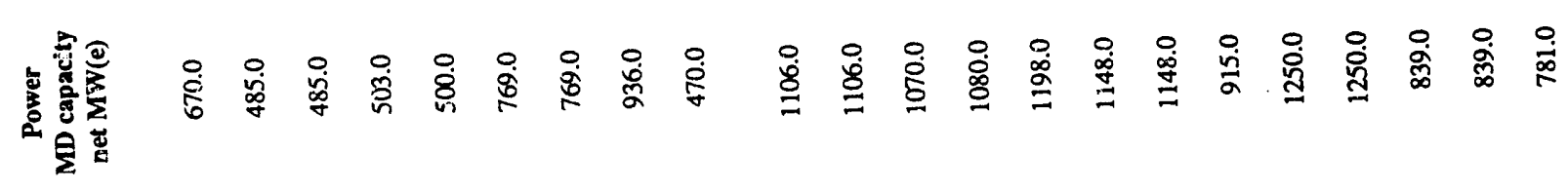

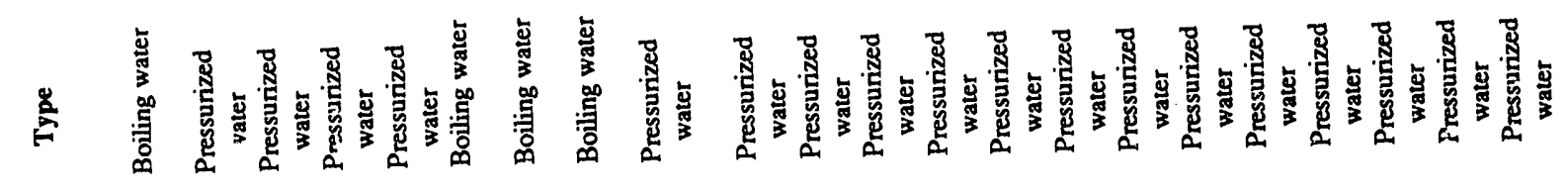

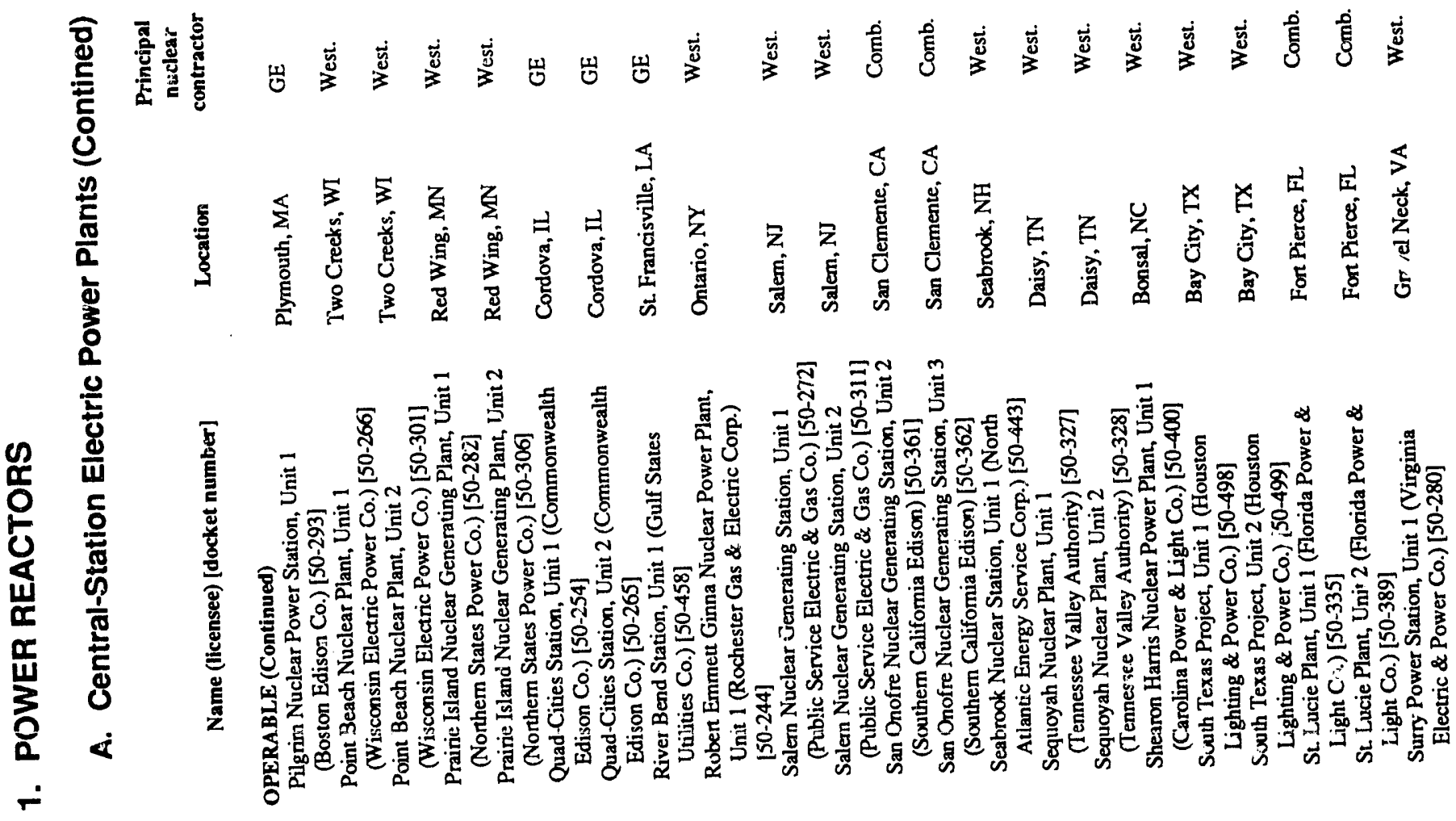



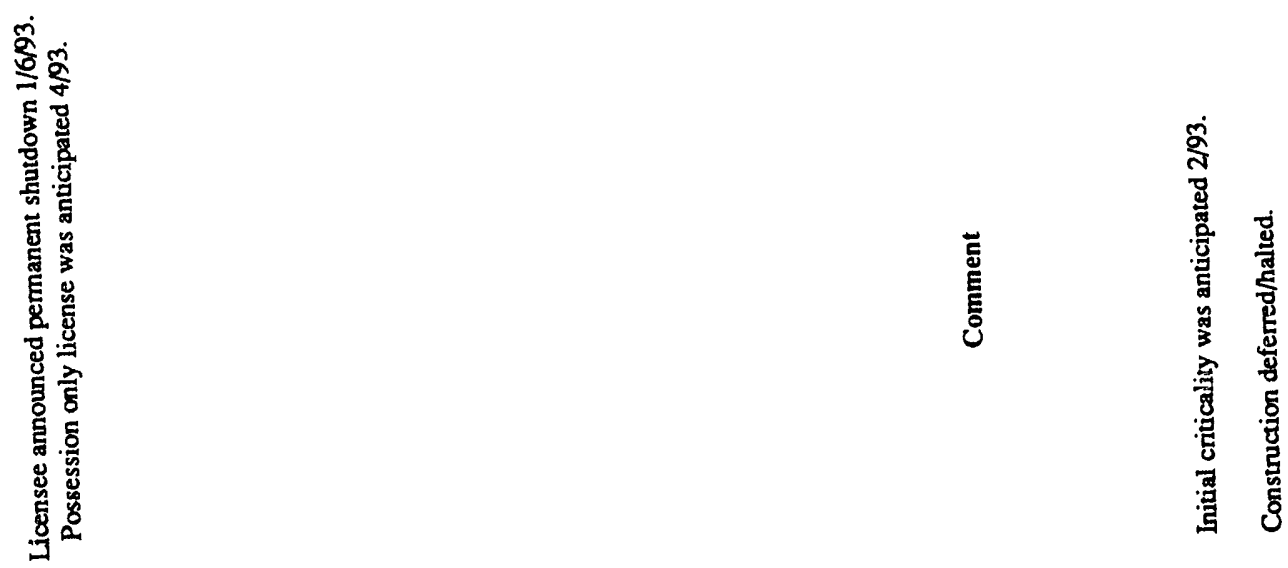

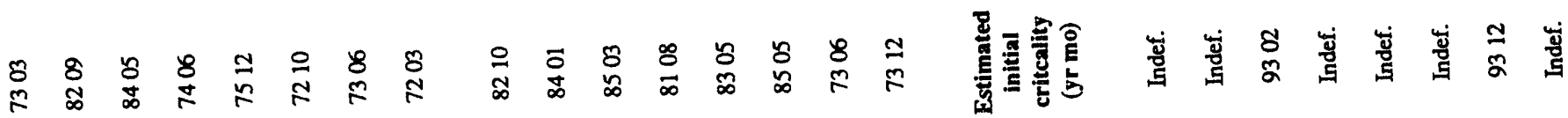

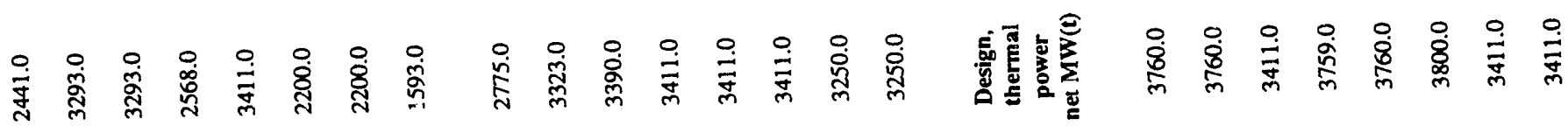

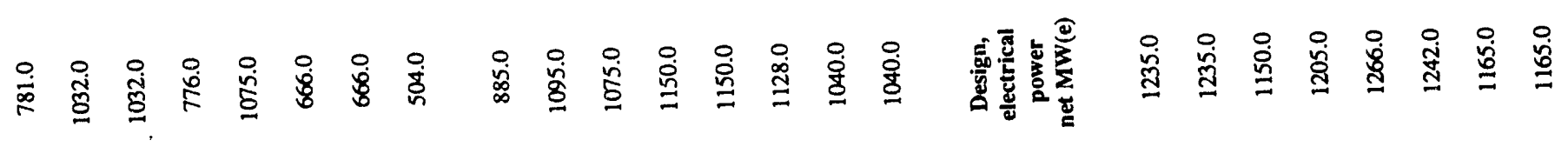
IIIIIn

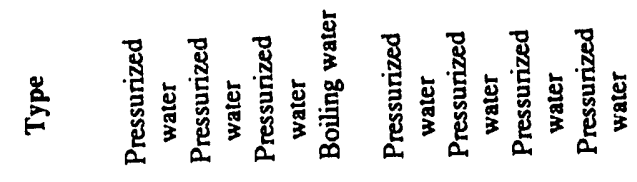

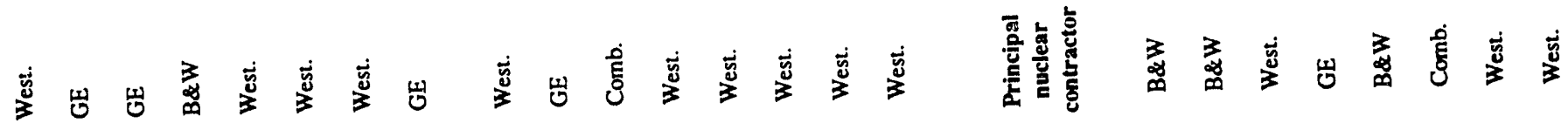

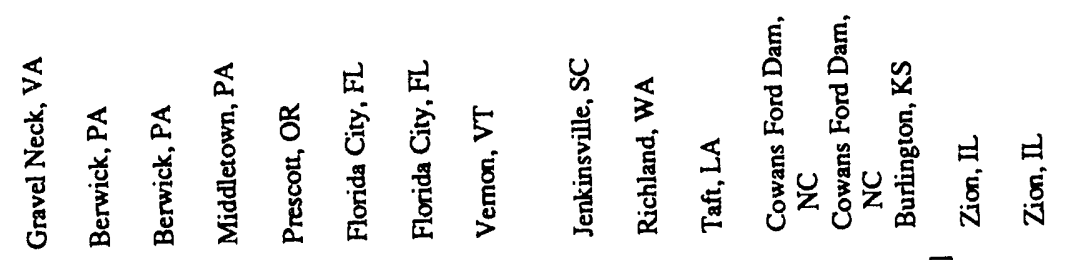

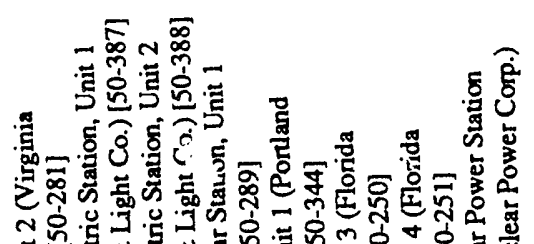

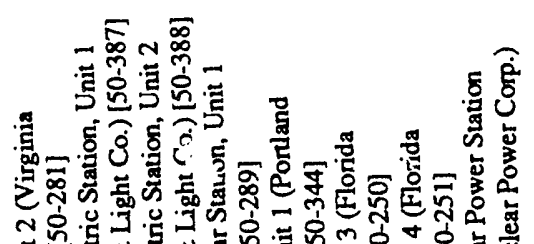

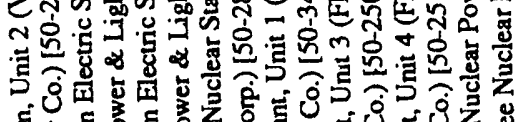

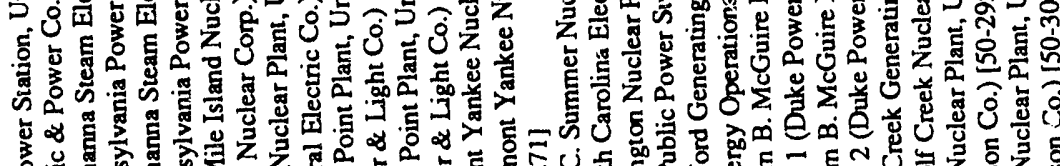

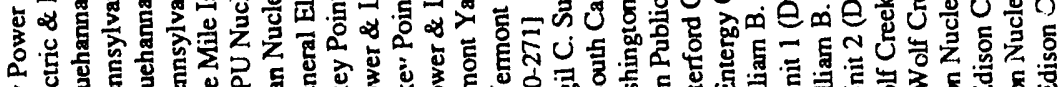

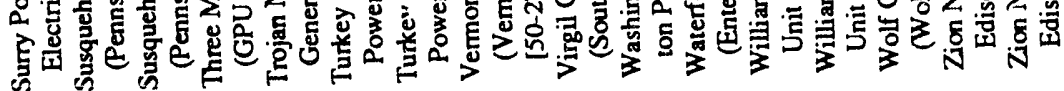

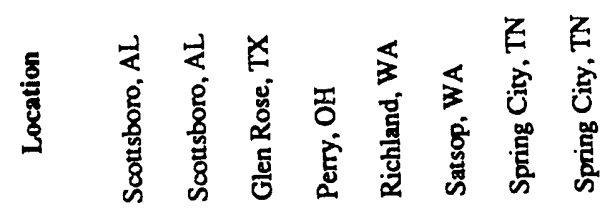

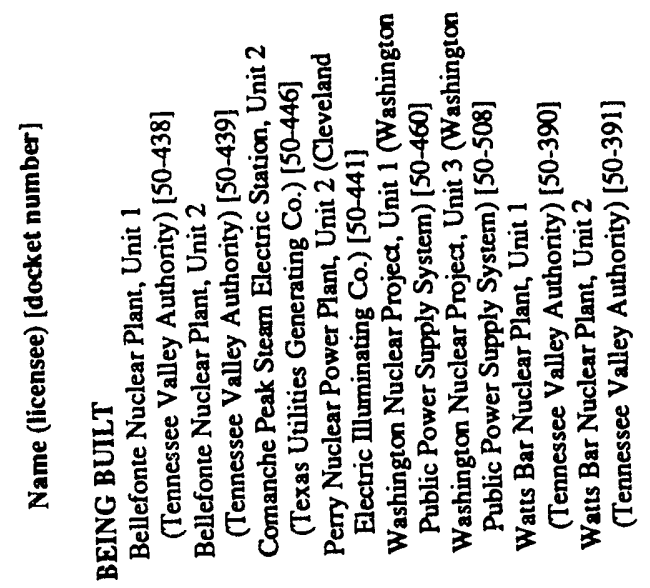




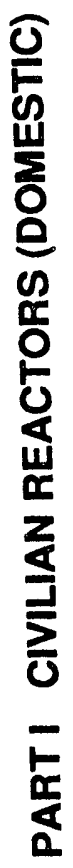
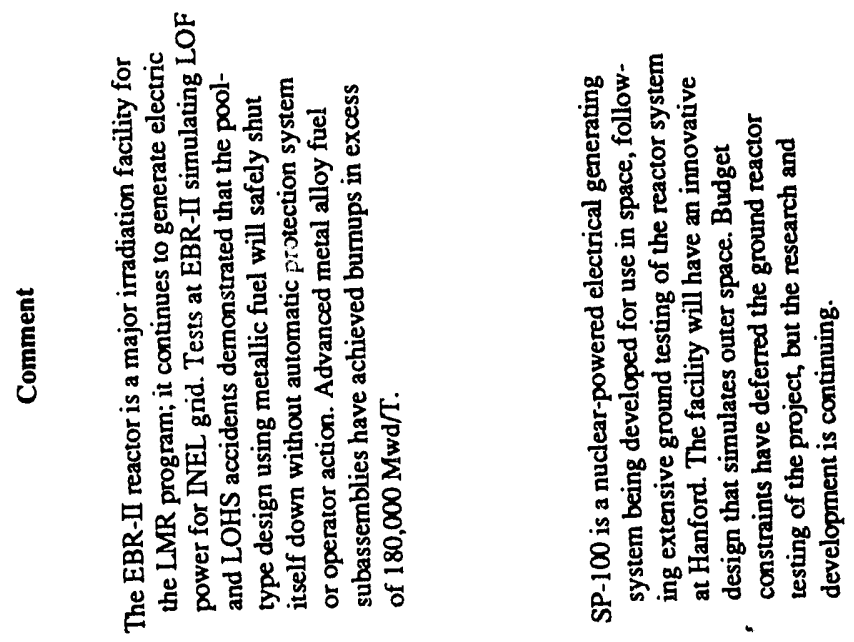

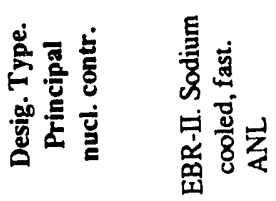

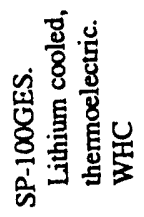

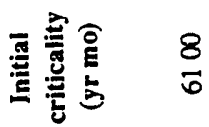

8
\&

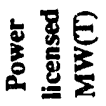

冚兽

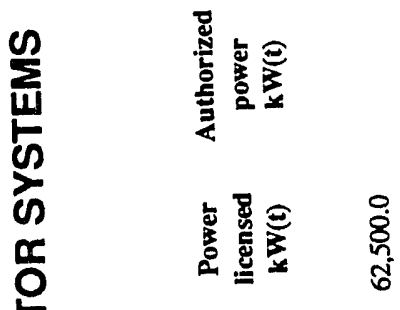

인

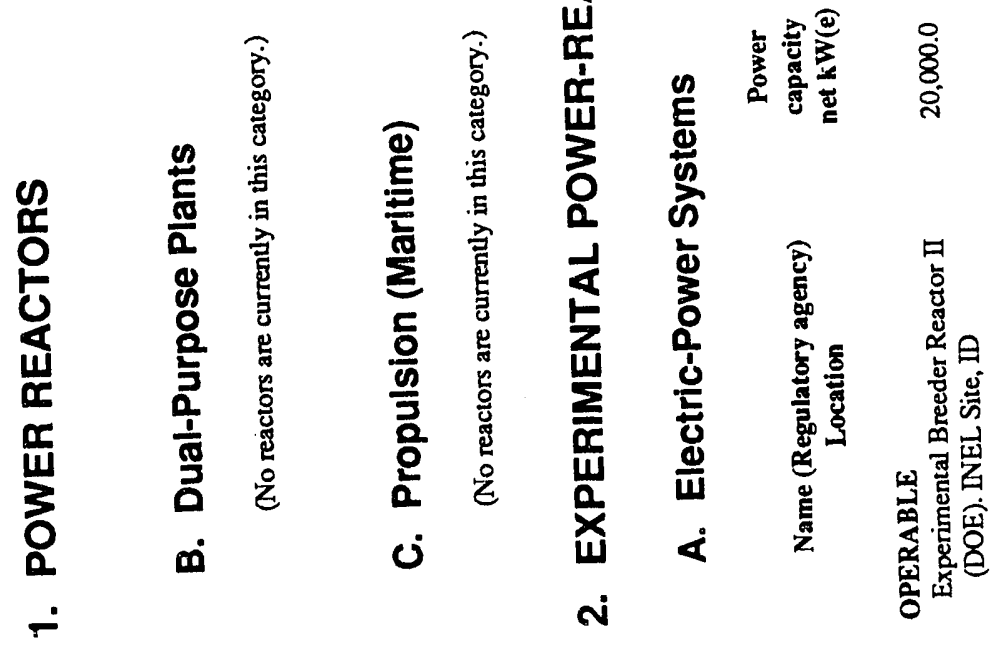

$\sum_{0}^{\frac{a}{a}} \stackrel{n}{0}$

产

$\frac{1}{2}$

文

石 密

के

क 

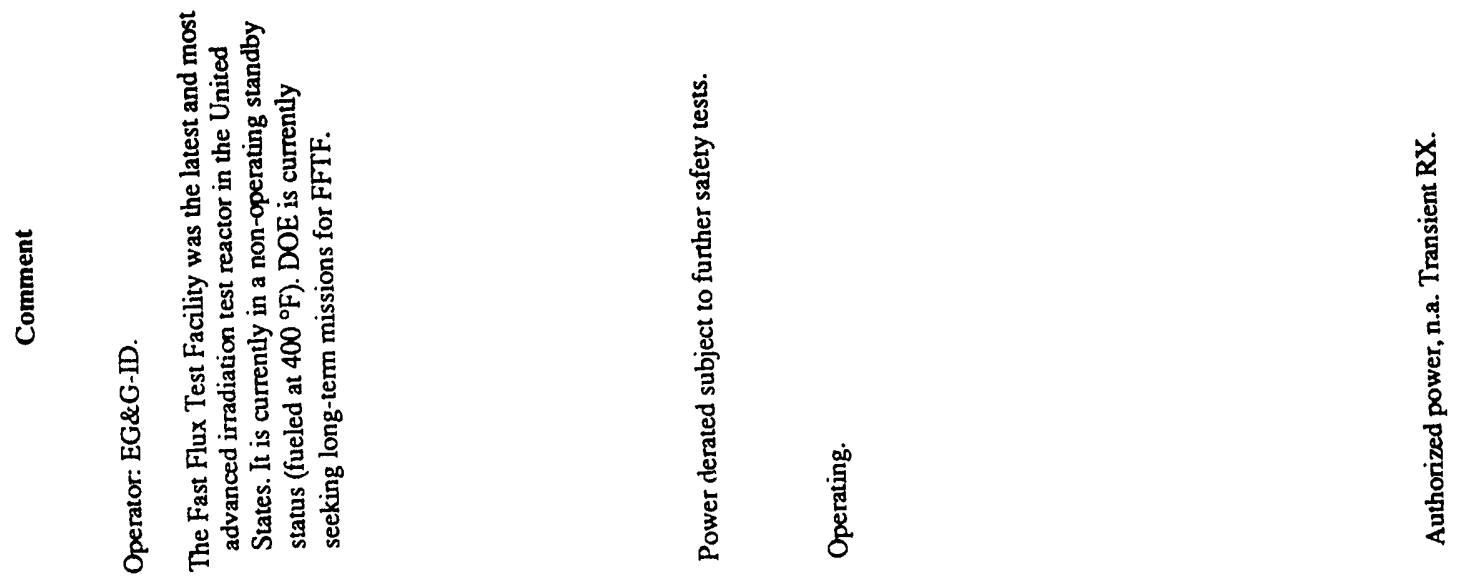

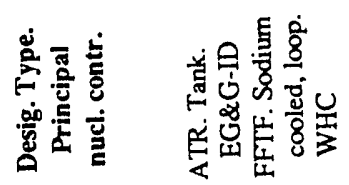
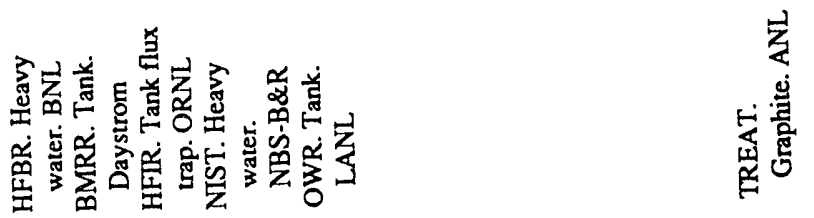

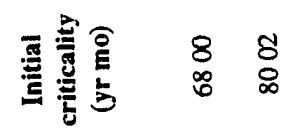

$\begin{array}{lllll}8 & 8 & 8 & 8 & 8 \\ 3 & 5 & n & 5 & 0\end{array}$

8

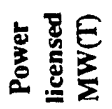

突密

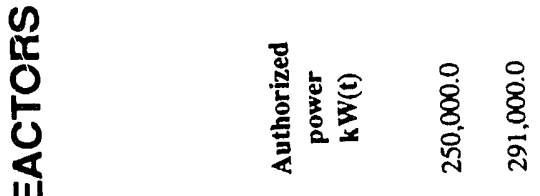

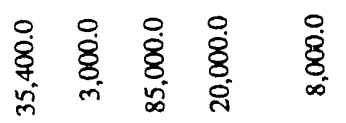

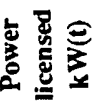

$\sum_{0}$ क

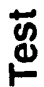

둥

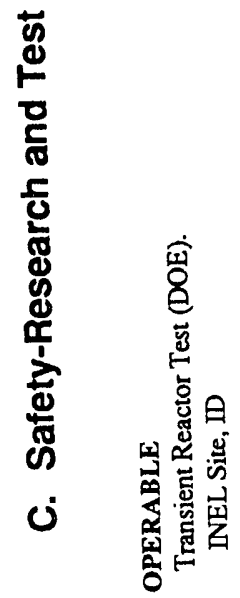

लं
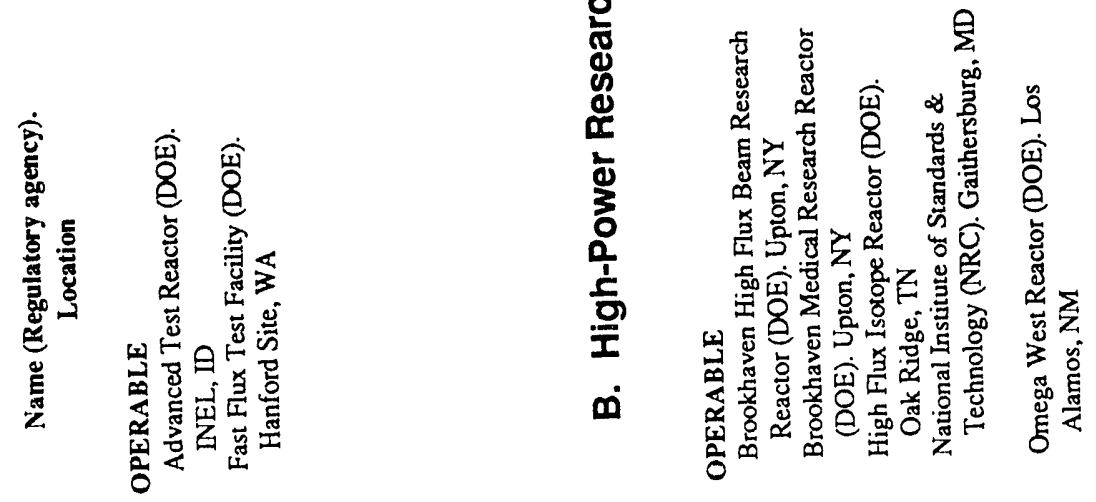


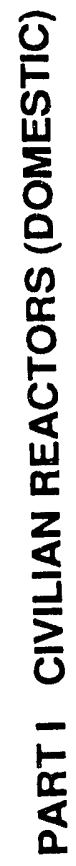
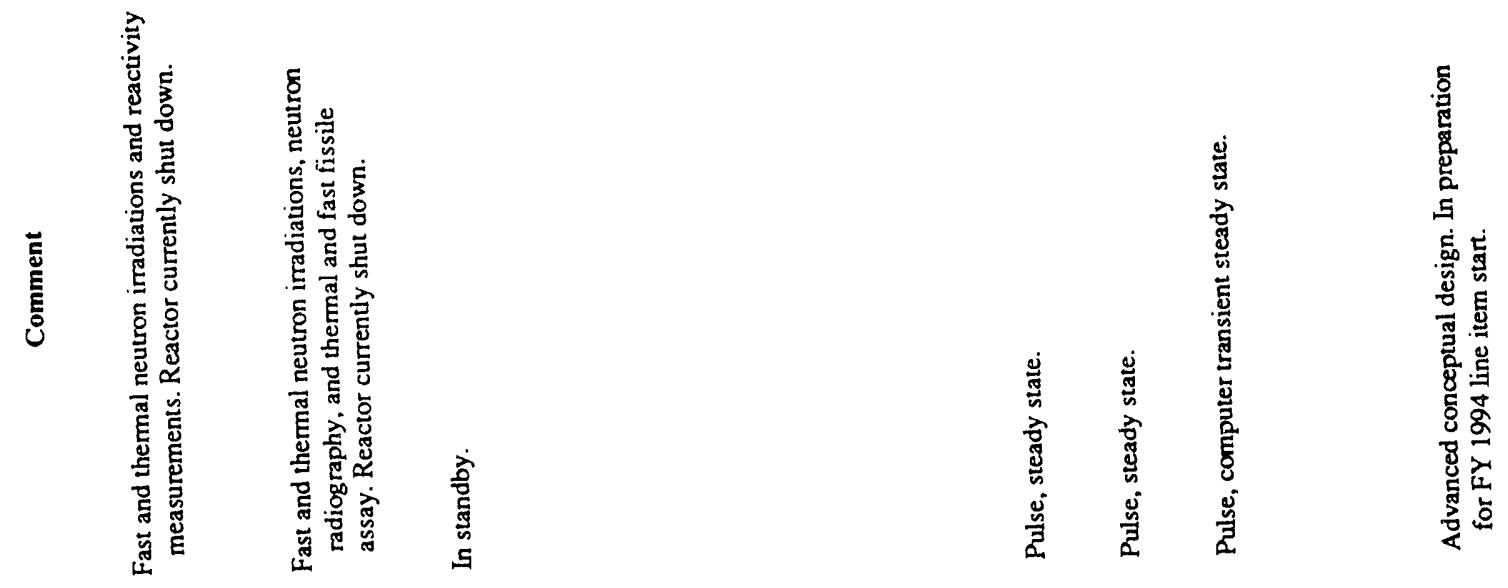

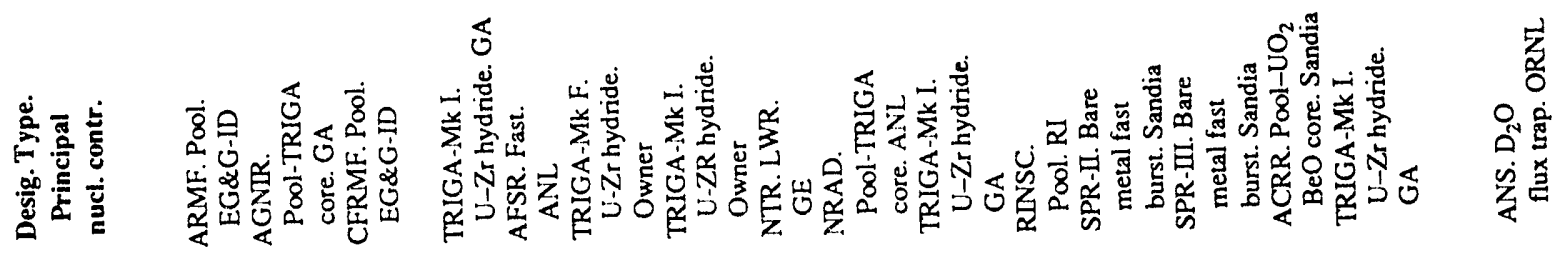

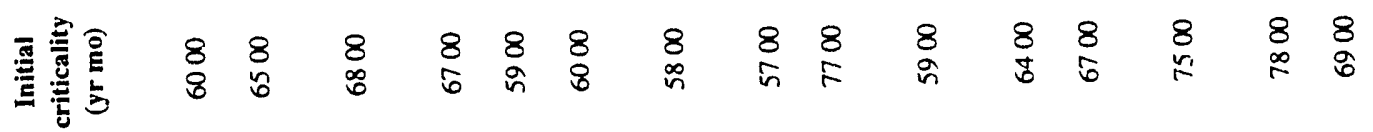

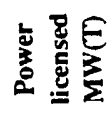

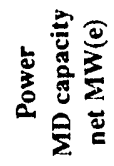

宽

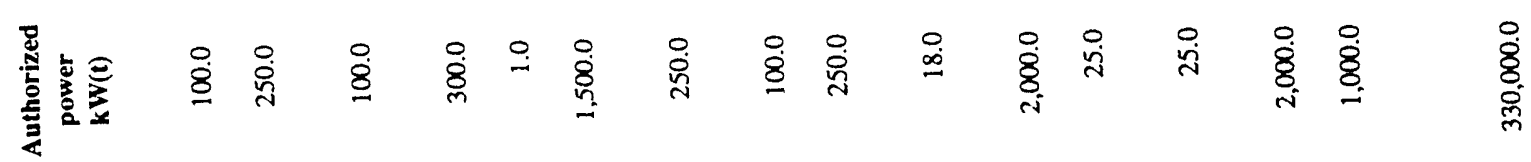
$\ln$

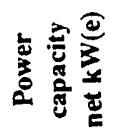

$\frac{9}{4}$

志

5

$\stackrel{m}{\square} \dot{0}$

$\dot{m}$

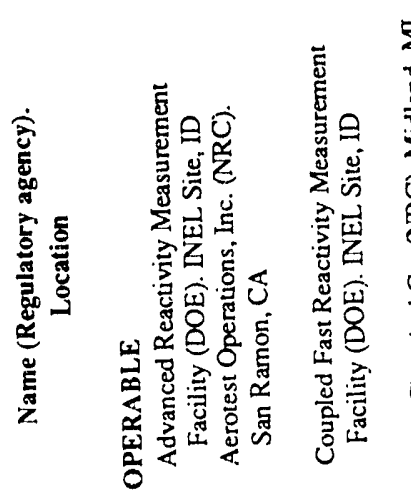

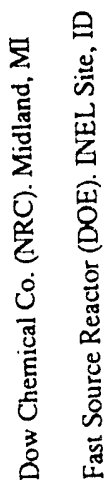

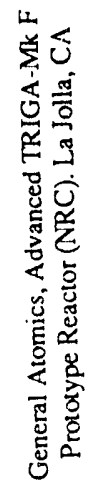

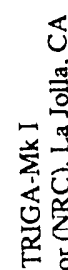

啚

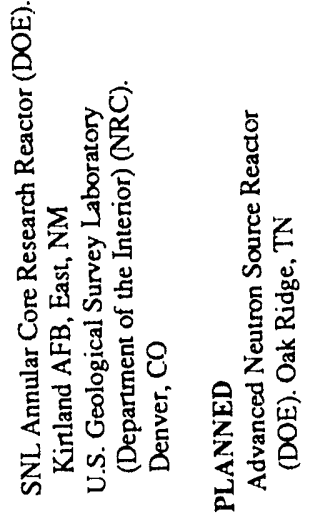



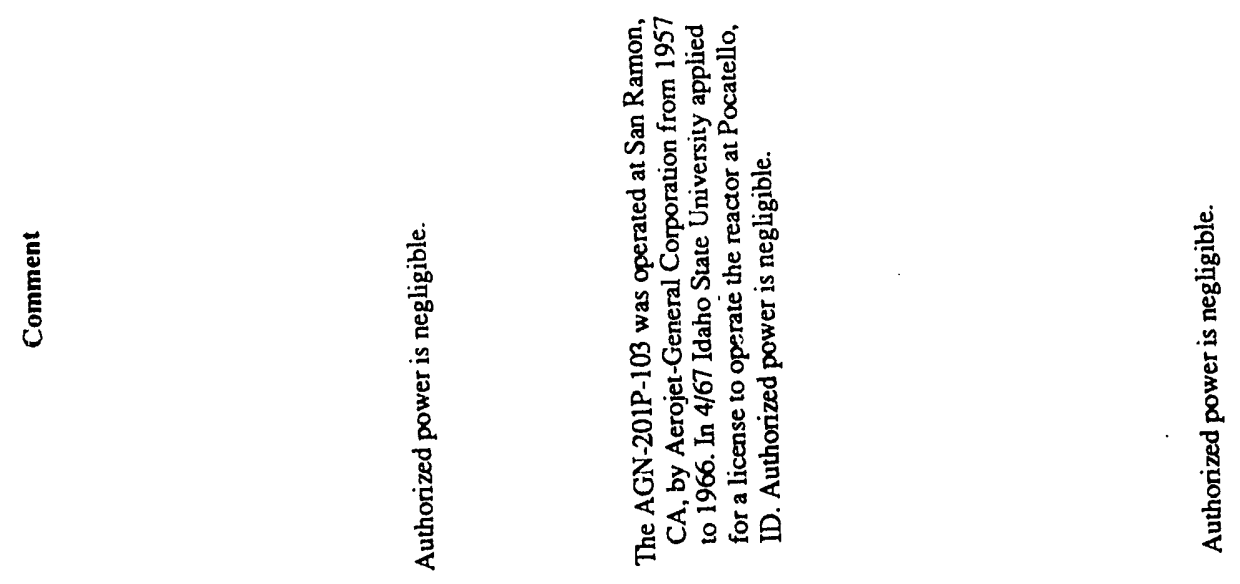

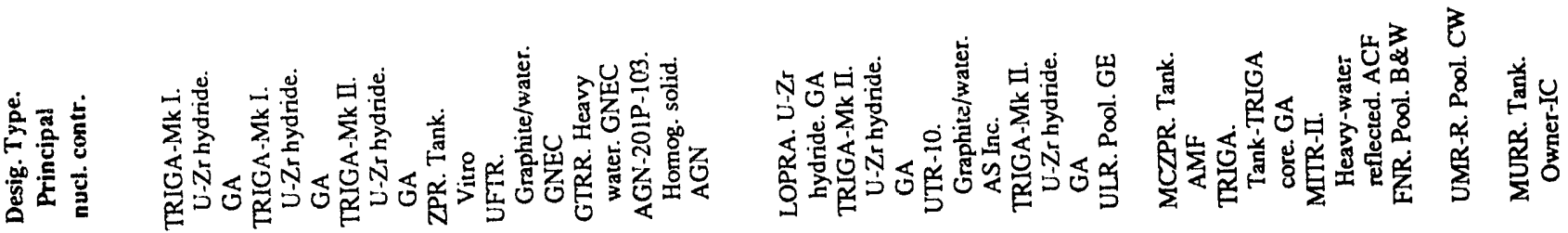

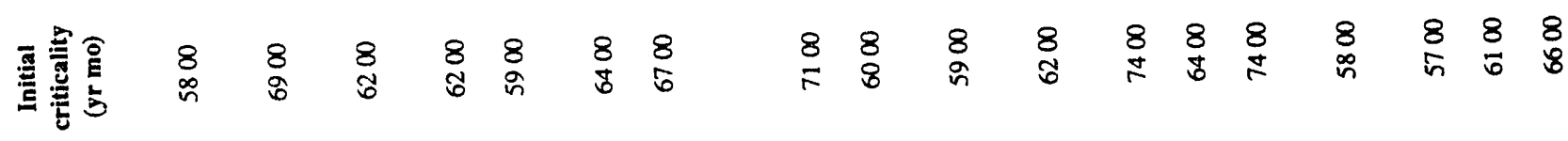
IIe

密总

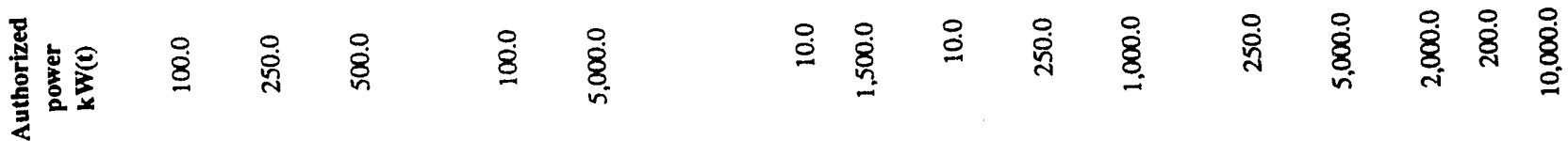

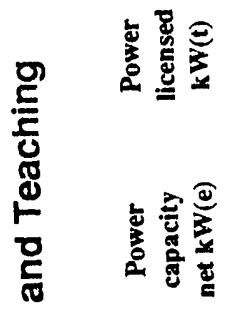

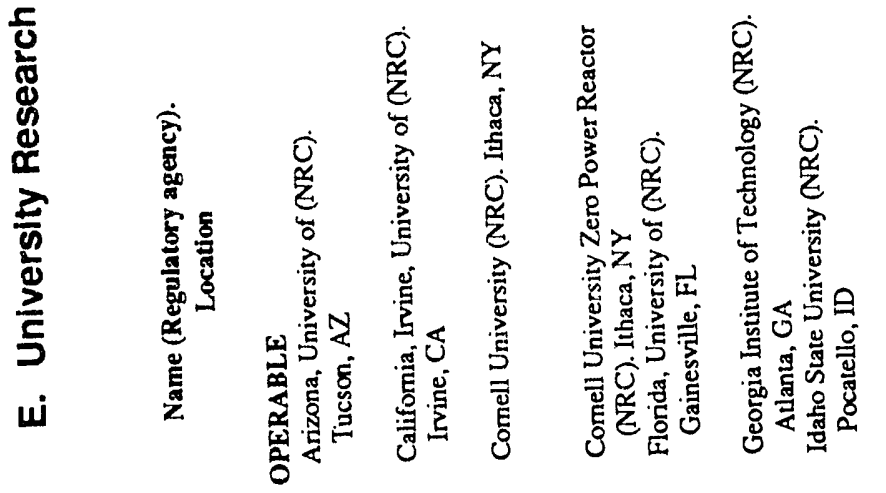
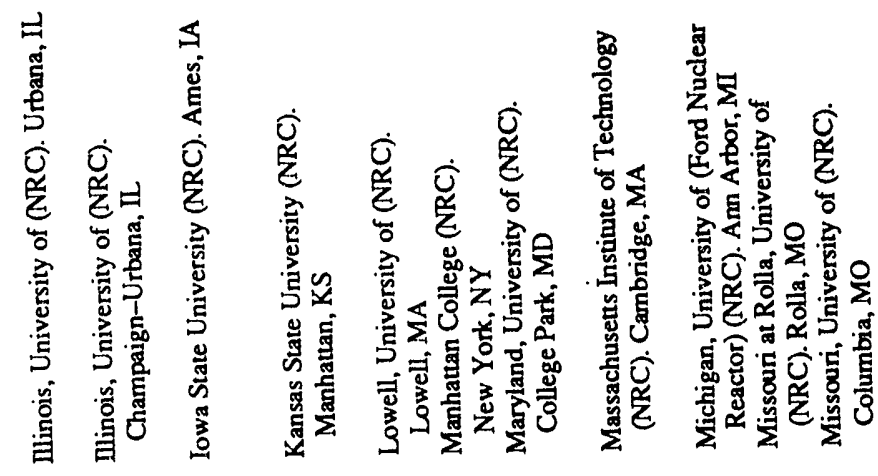


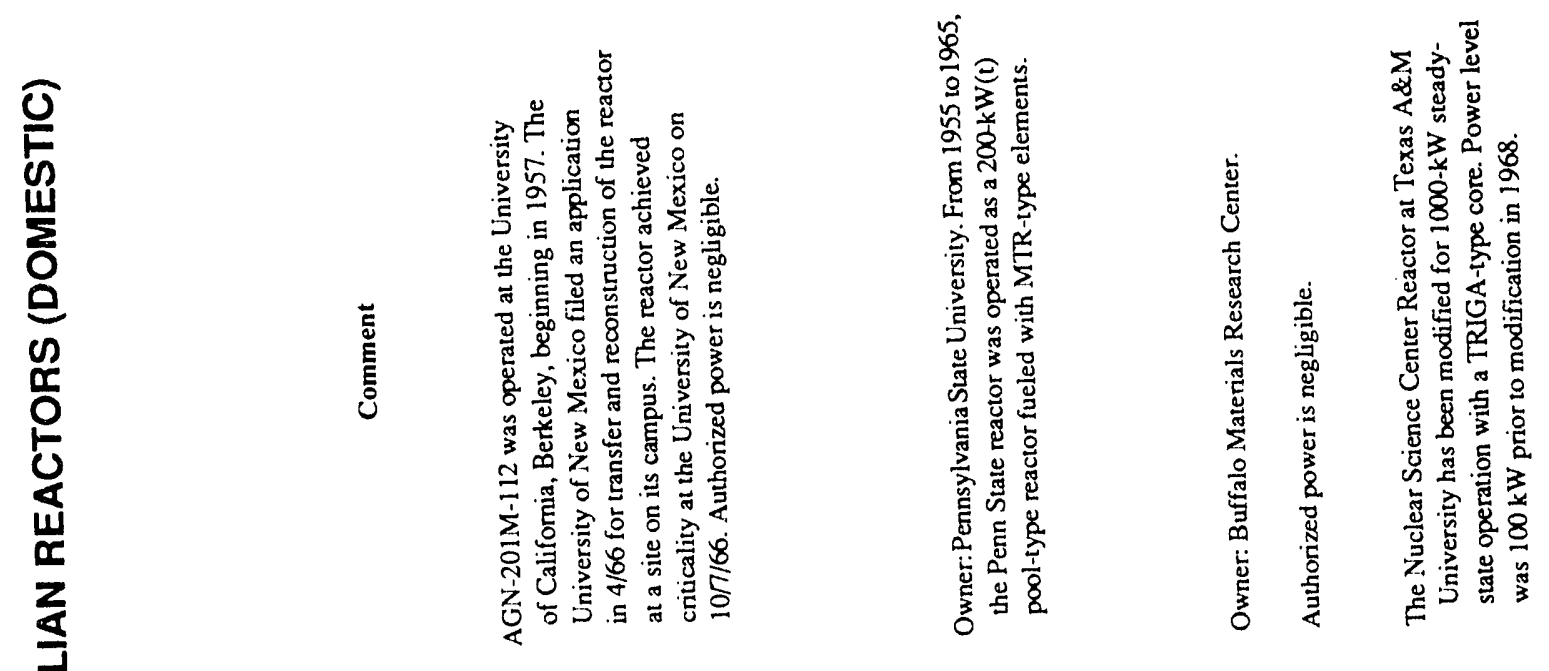

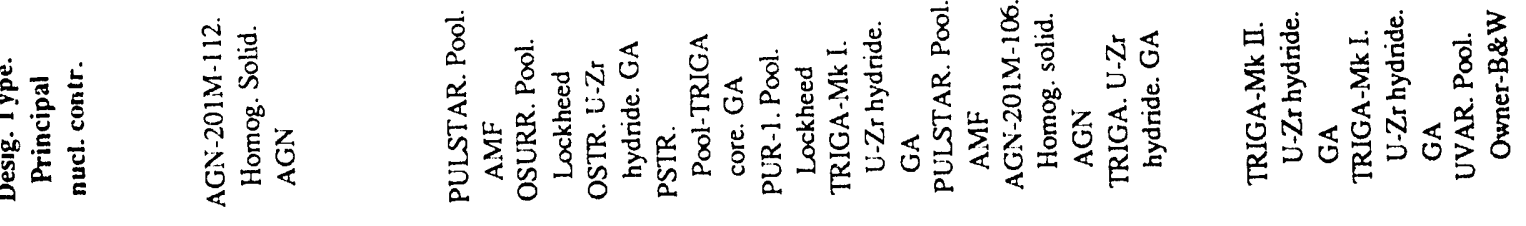

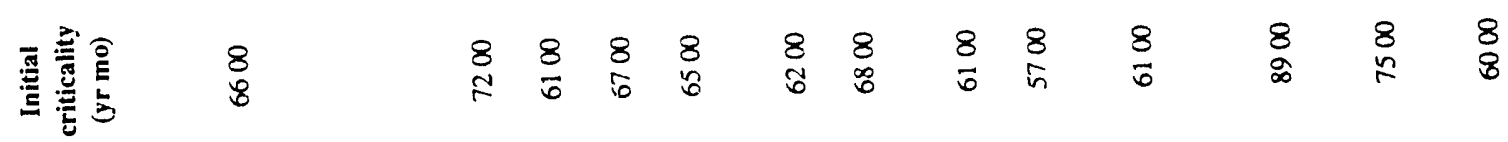

蹧量

总密

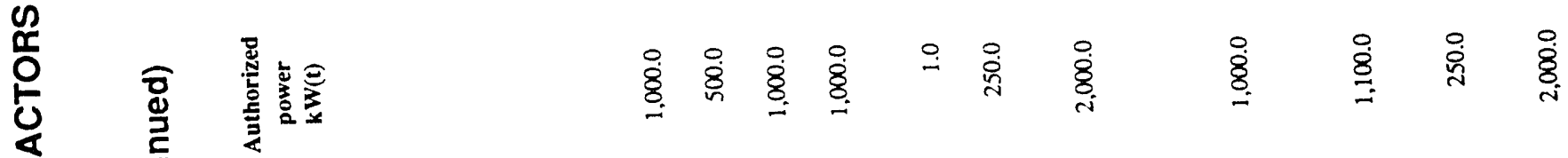

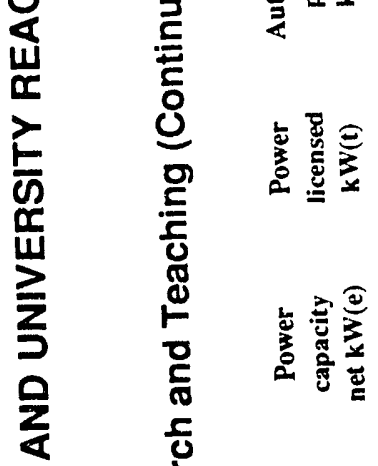

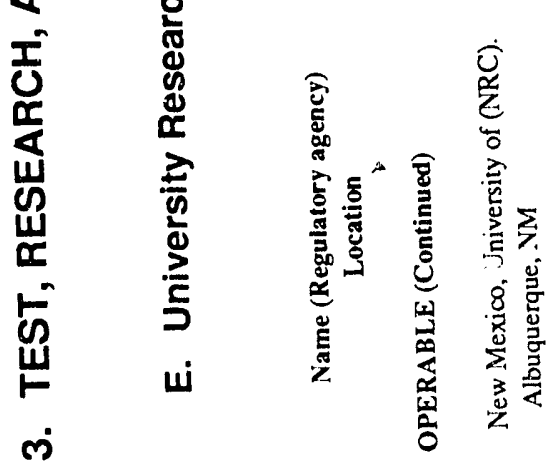

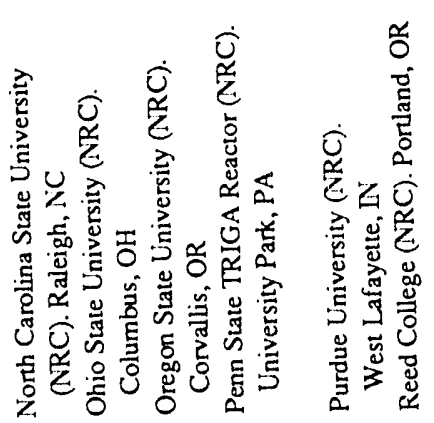

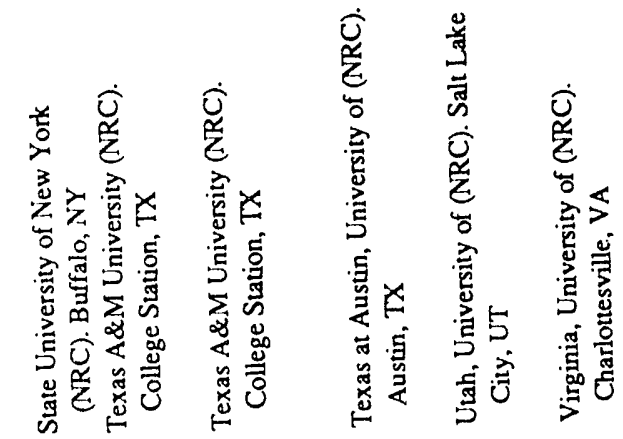



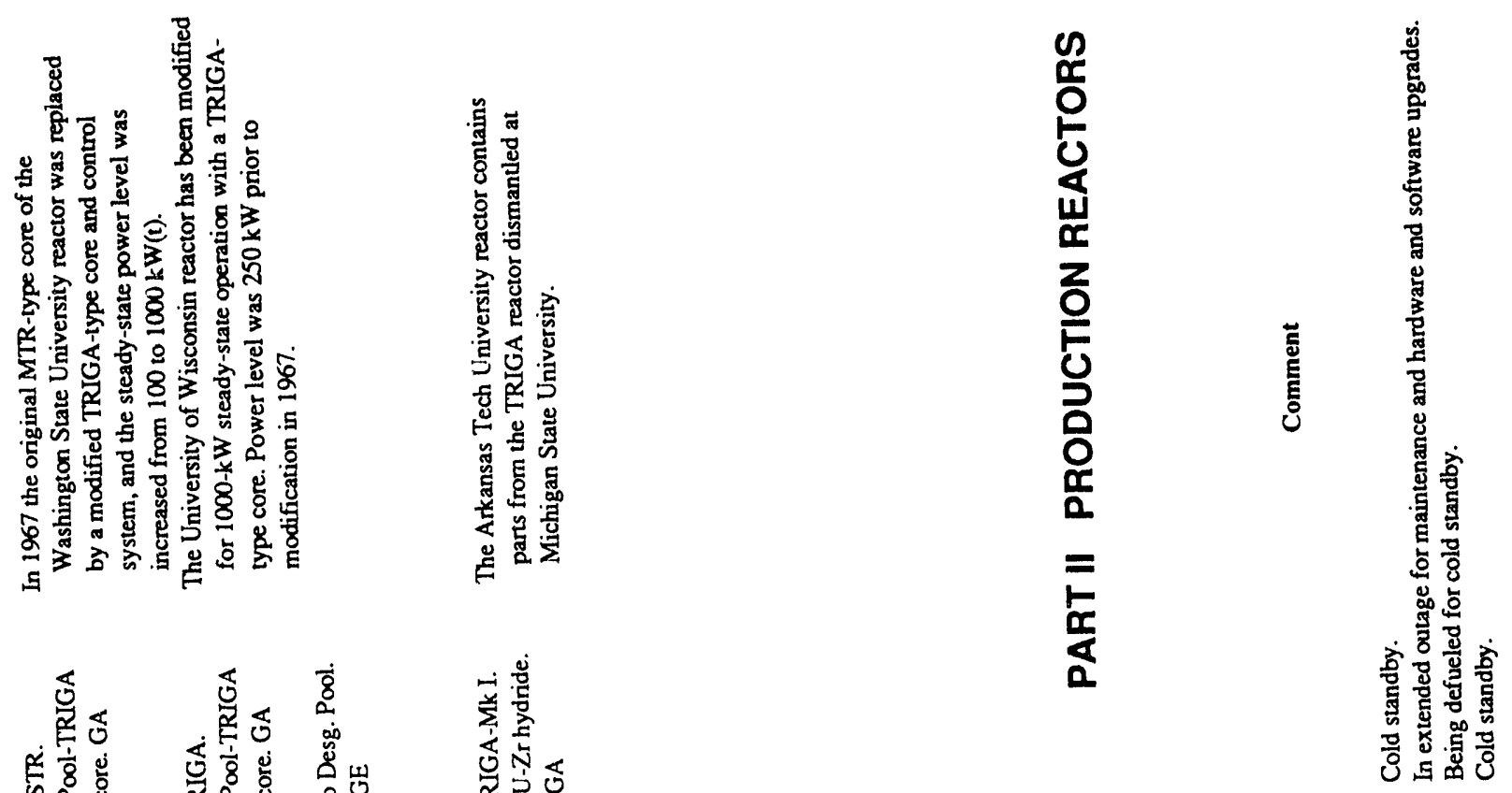

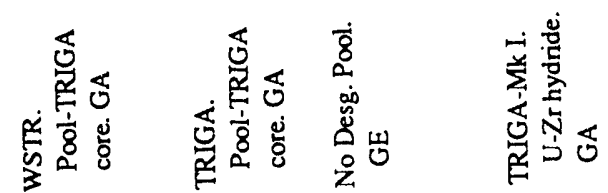

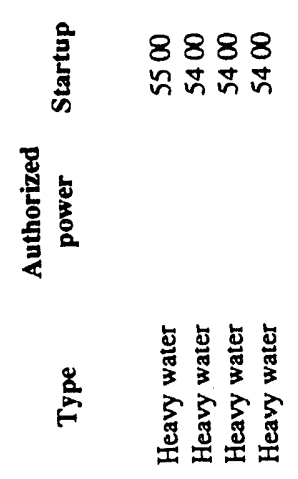

용 \&

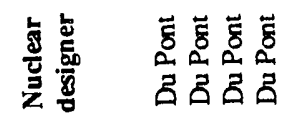
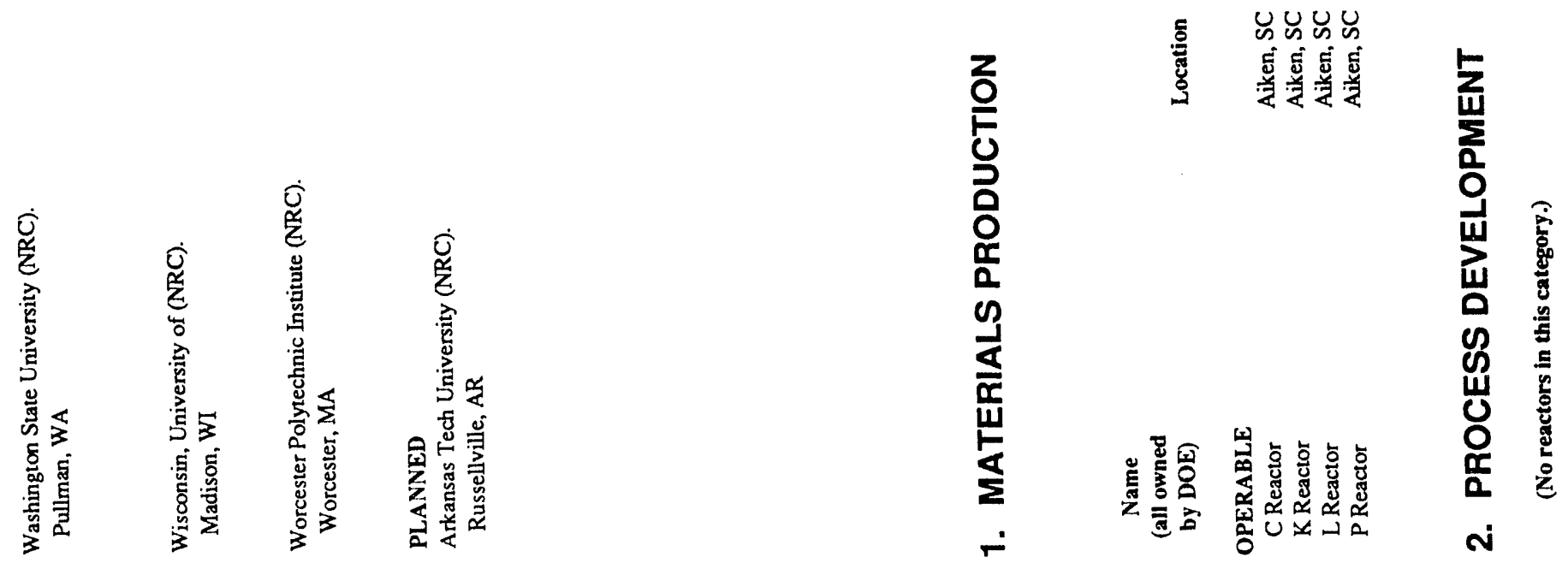


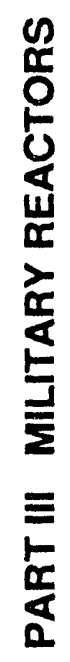

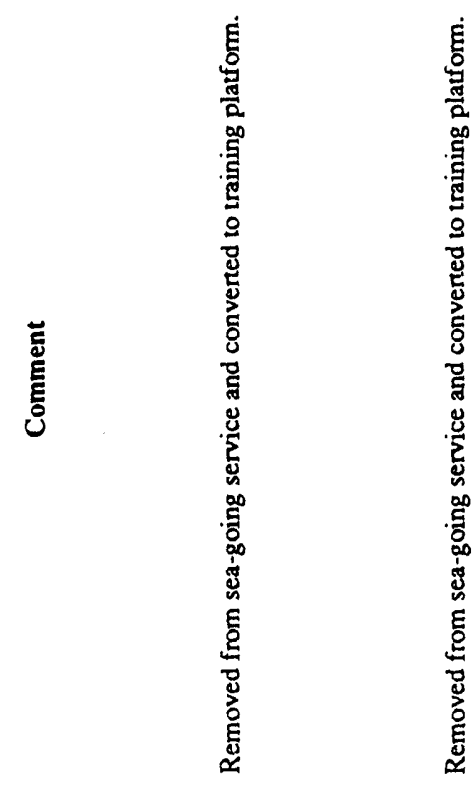

(2) 88888888888888888888888888888888888888 氛
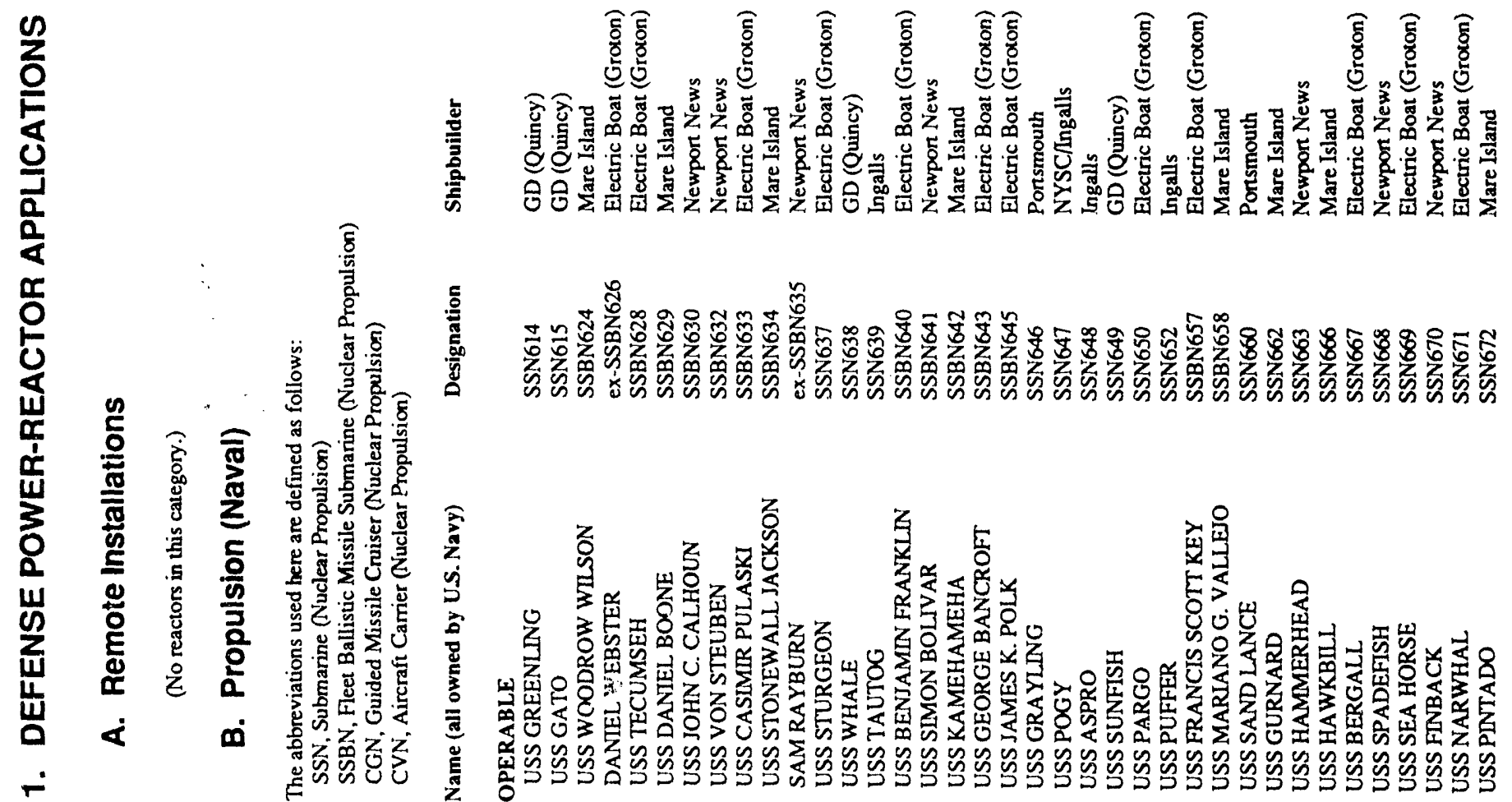

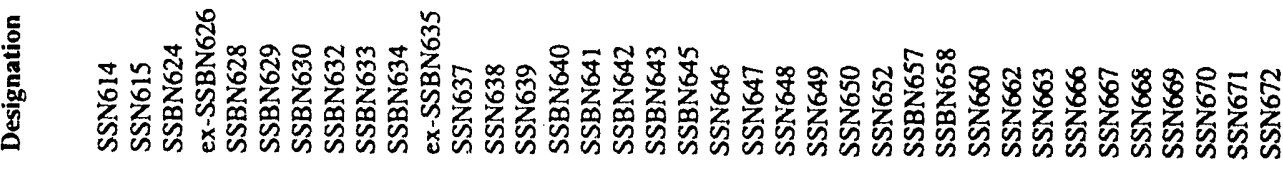
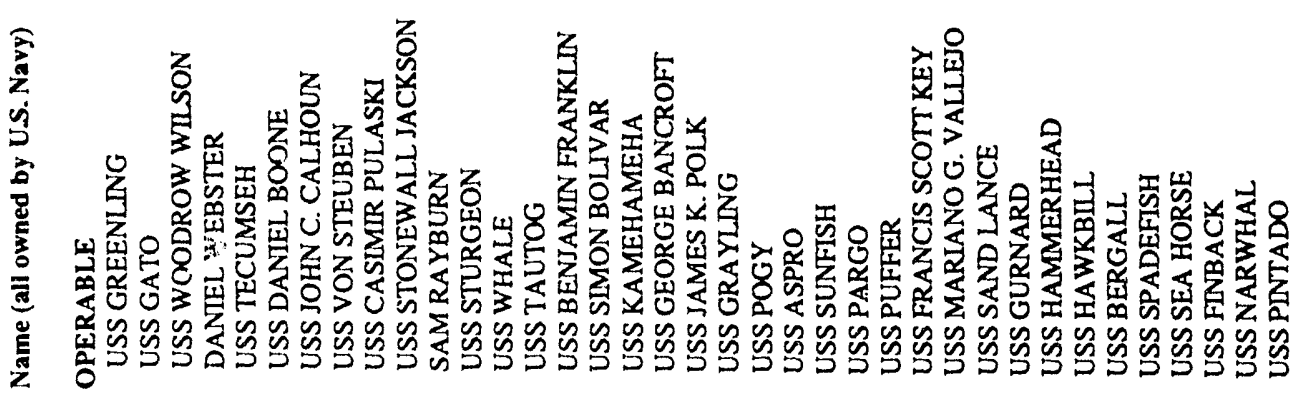


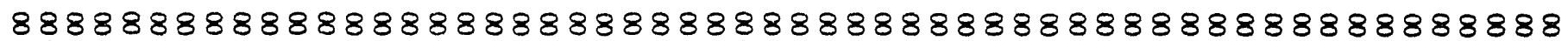

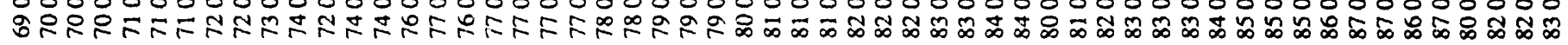

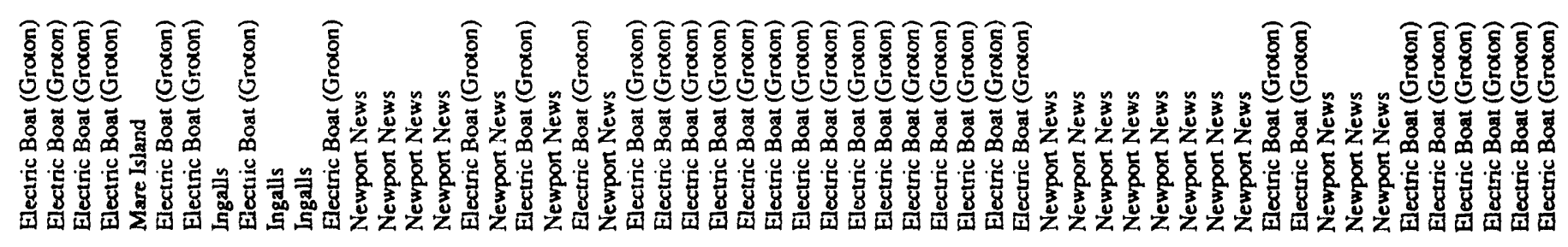

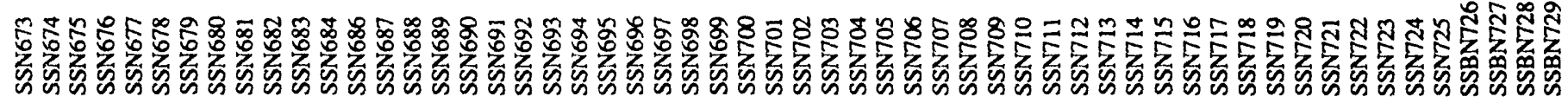

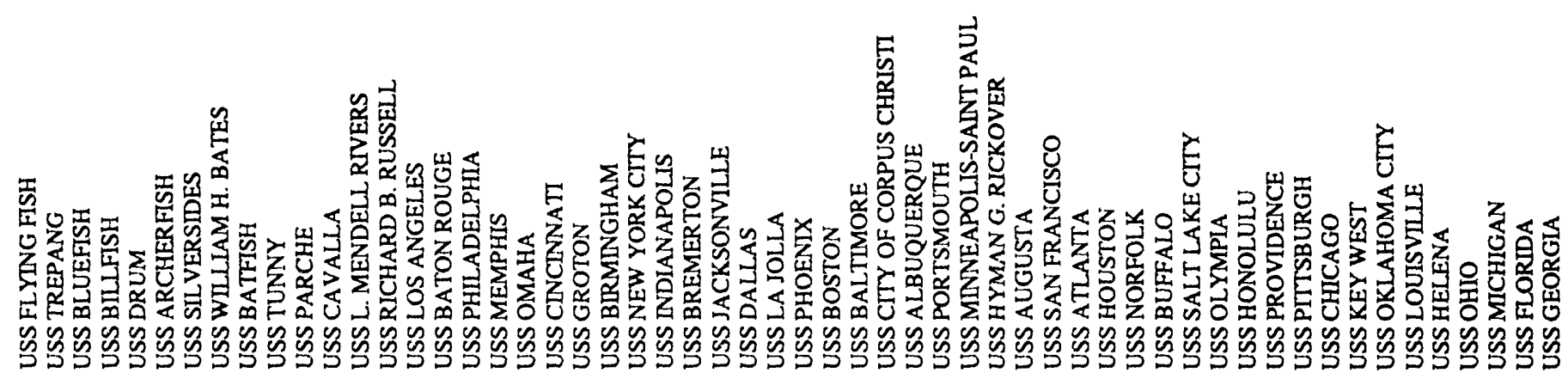




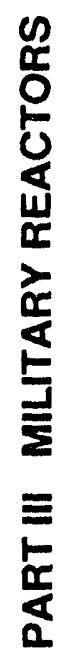

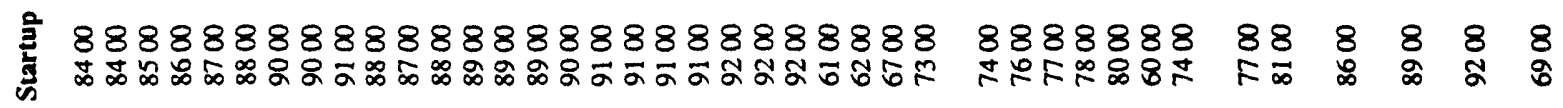
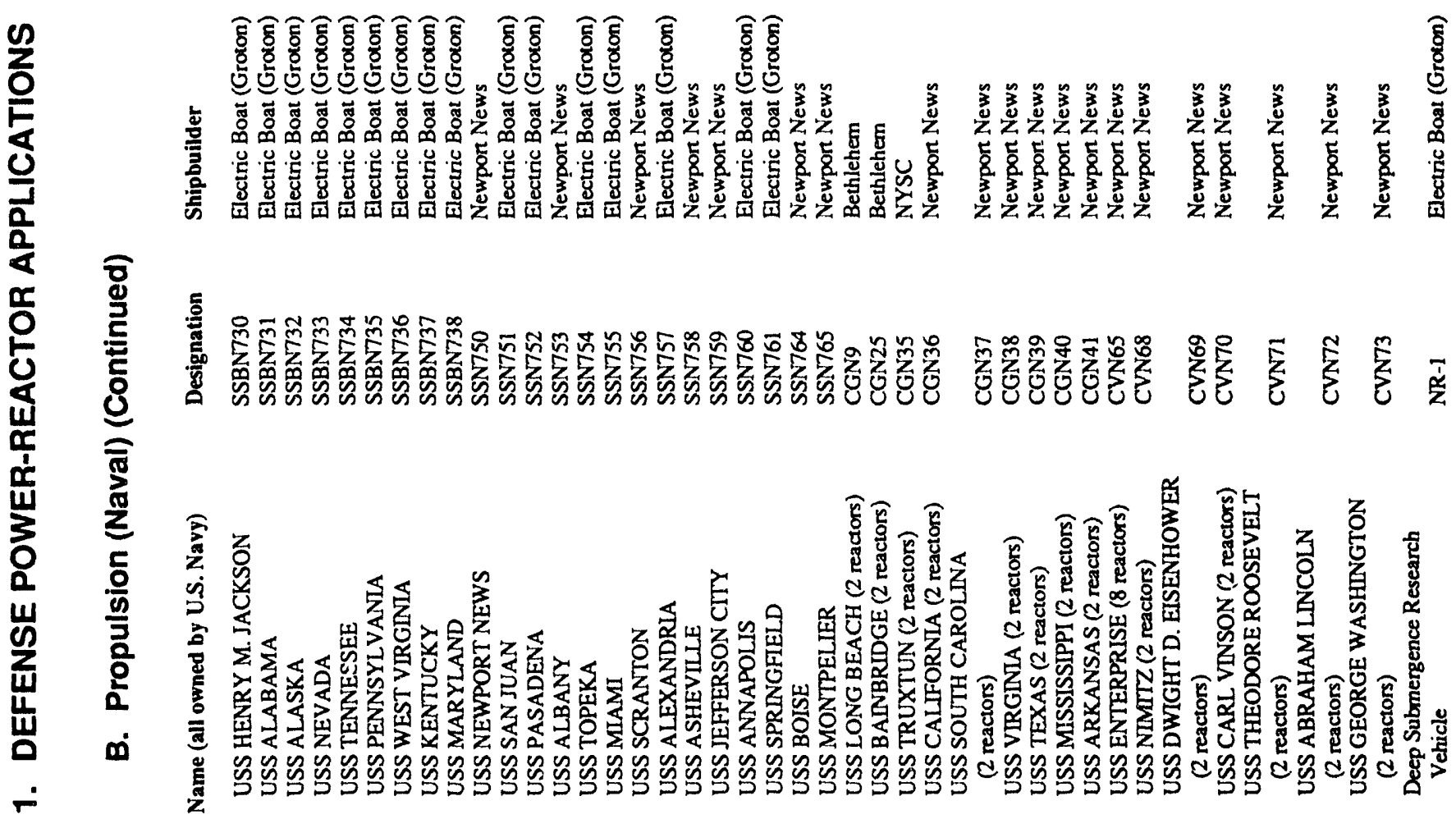


\section{I}

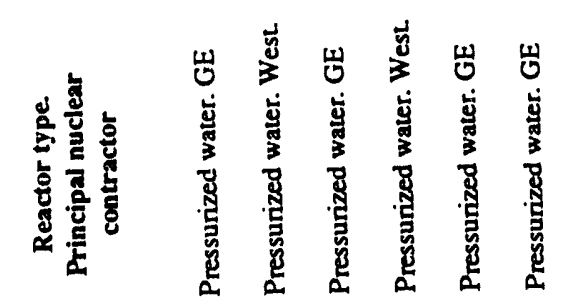

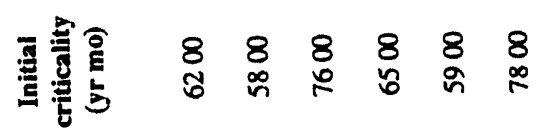

In

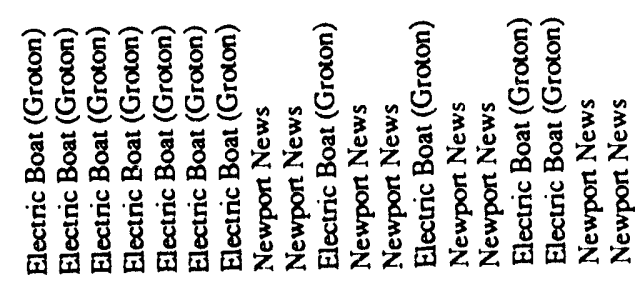

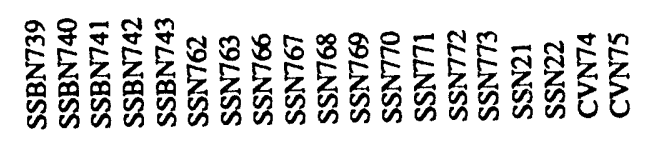

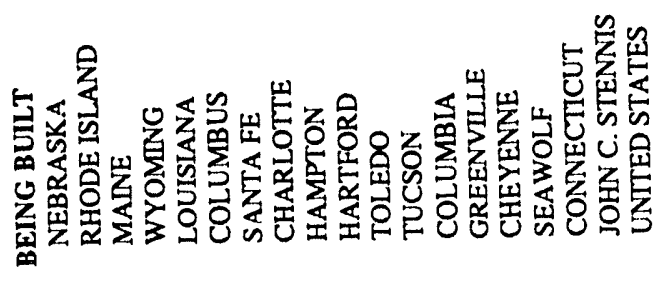

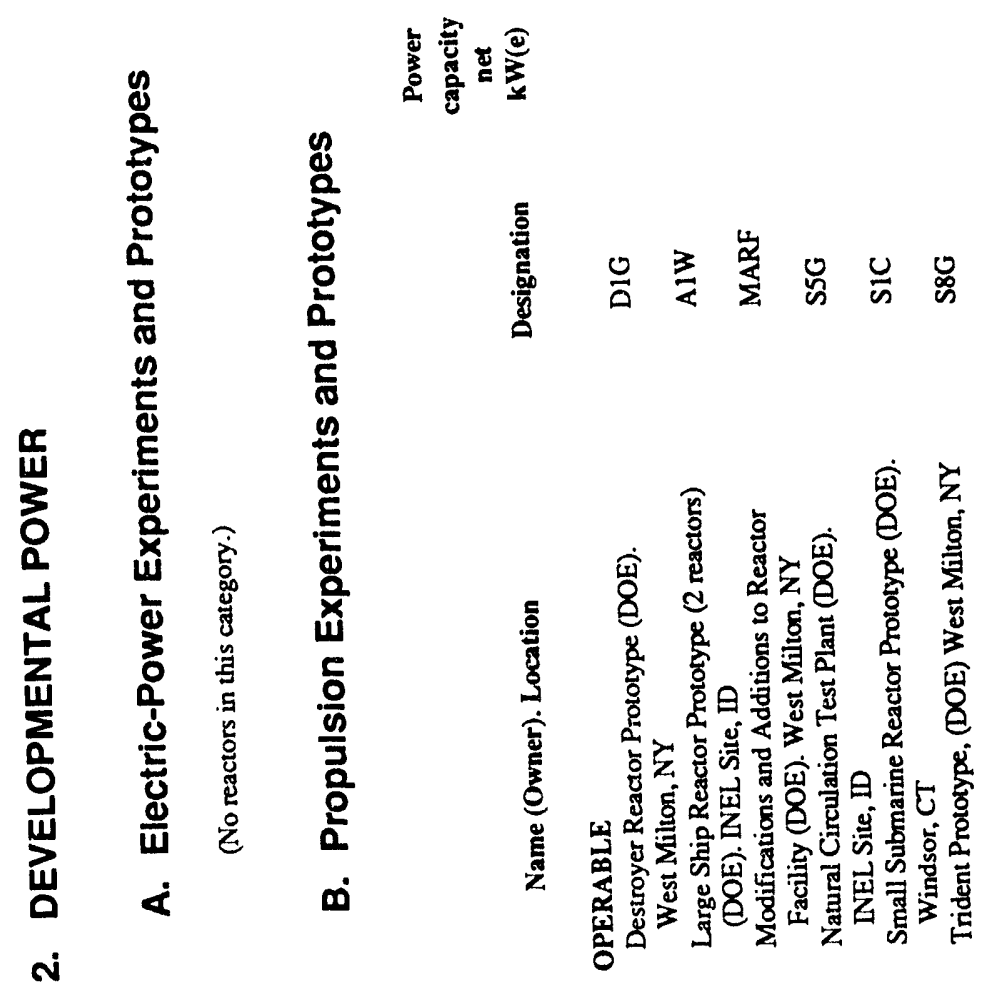




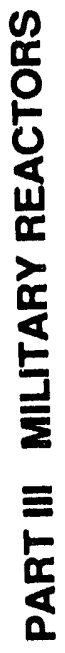

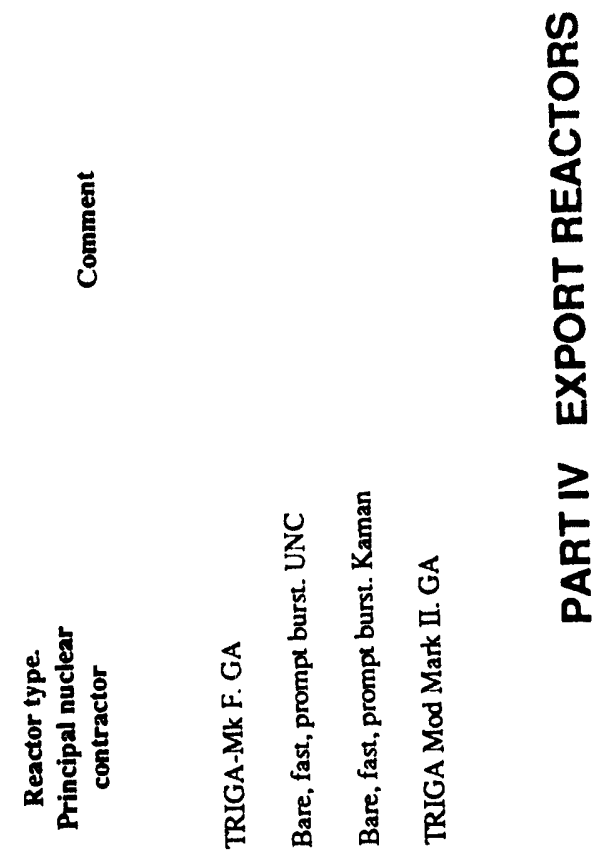

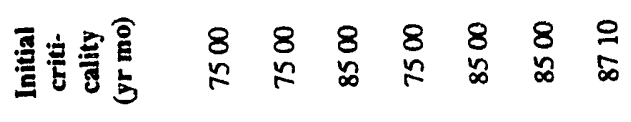

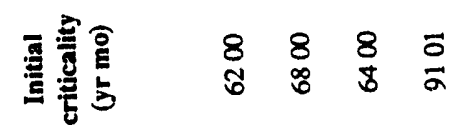

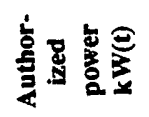

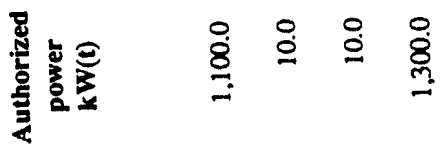

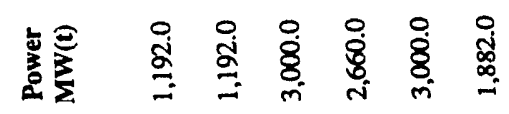

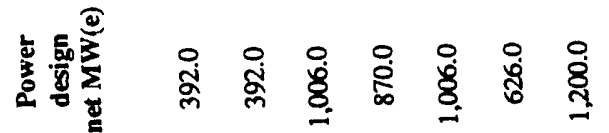

裹敦

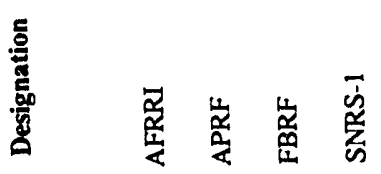

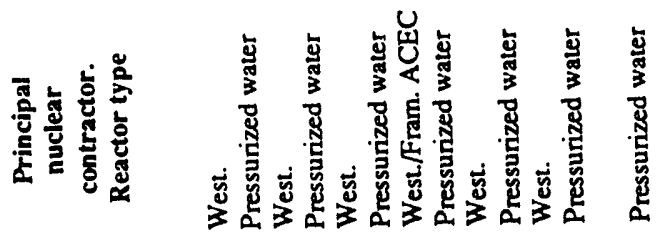

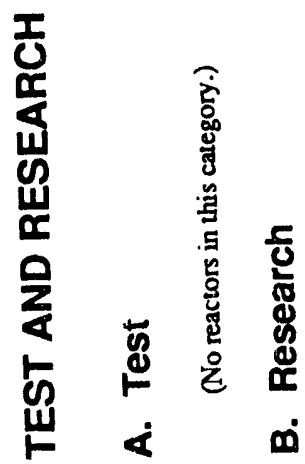

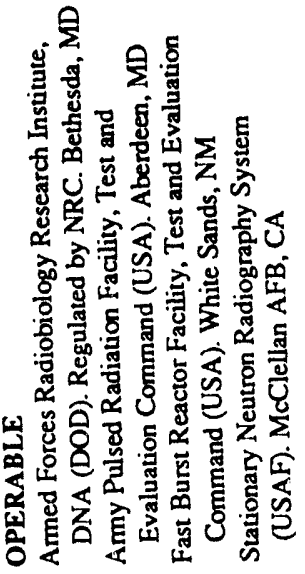

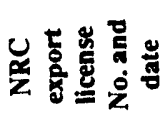

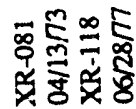

o

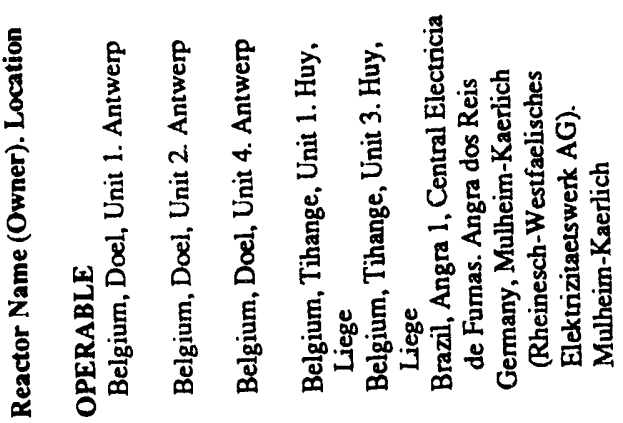



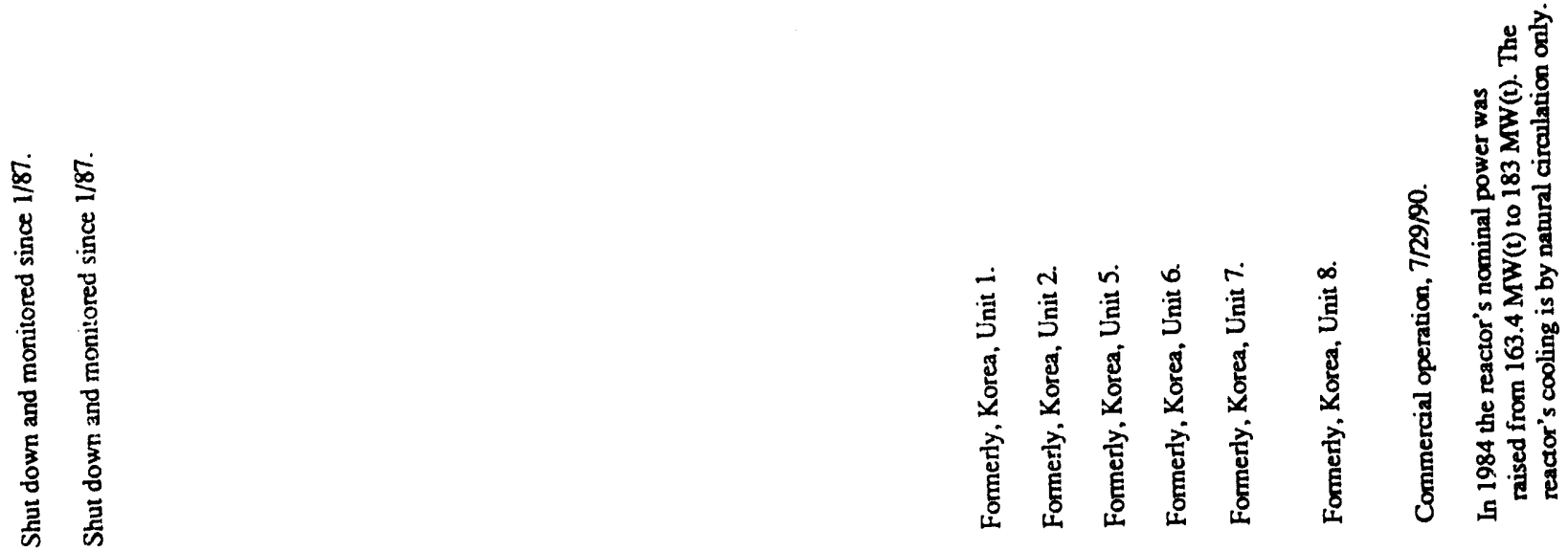

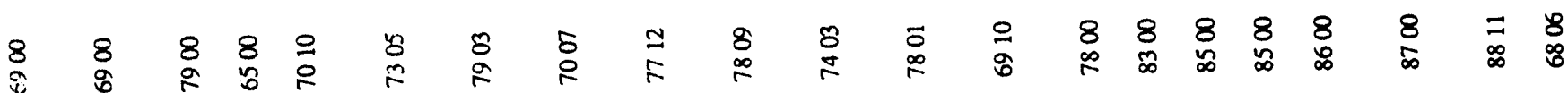

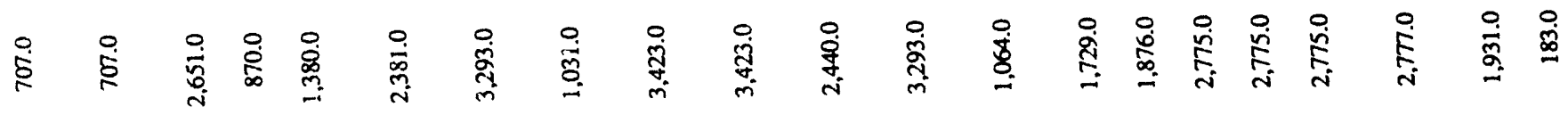
完

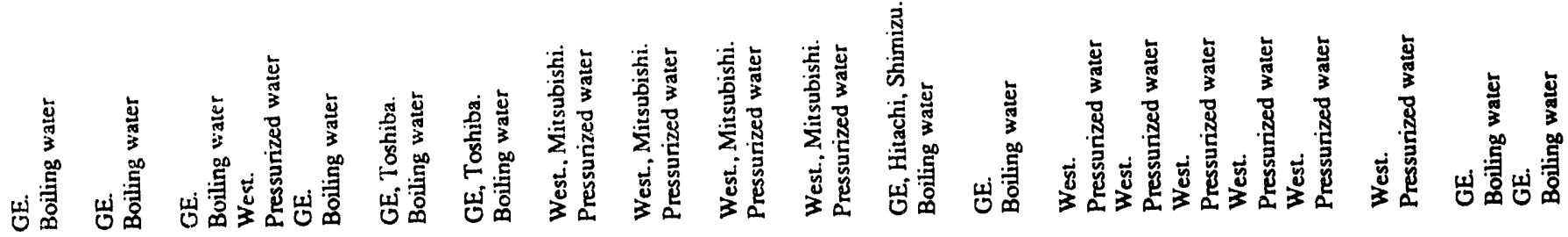

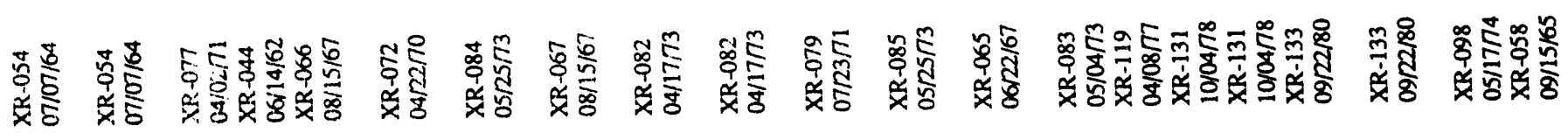

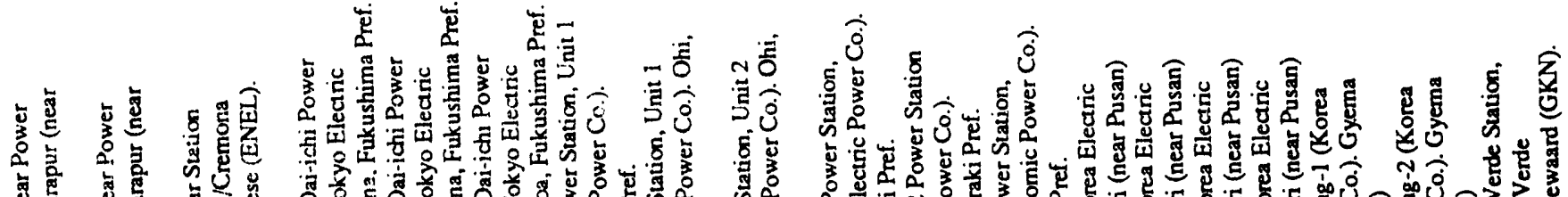

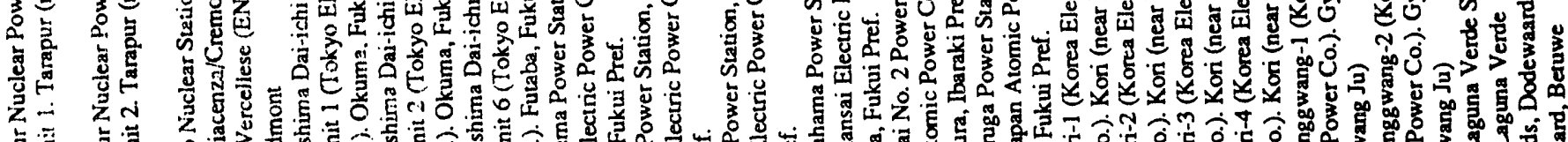

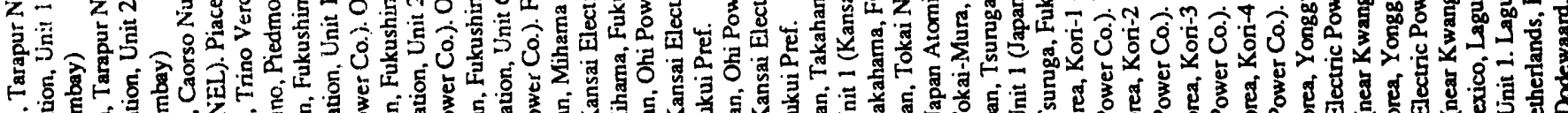

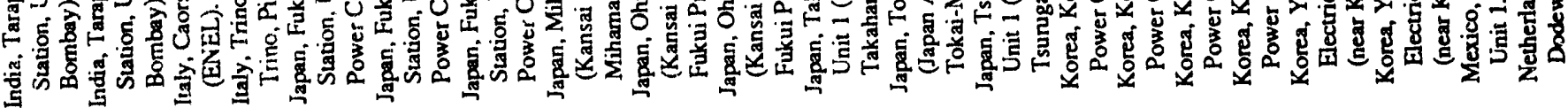




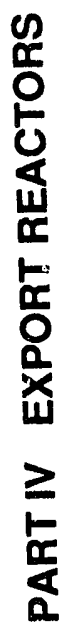

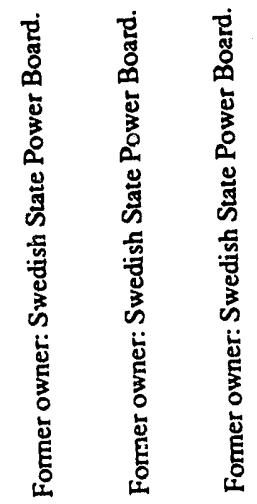

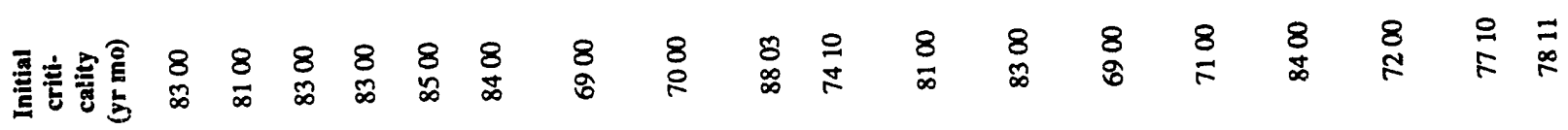

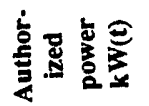

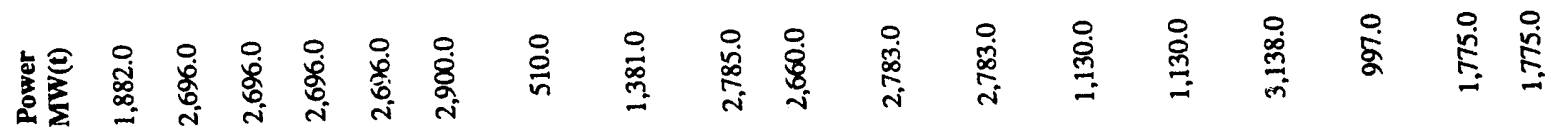

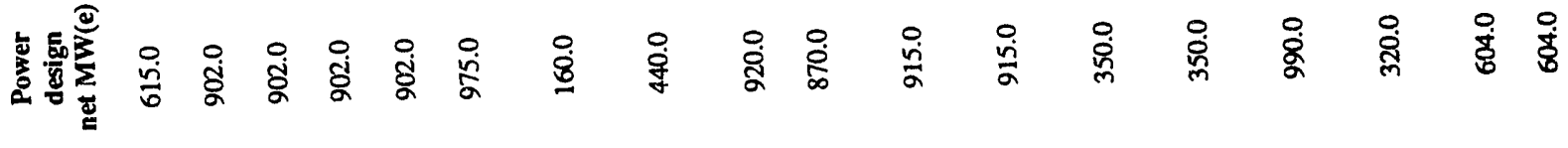

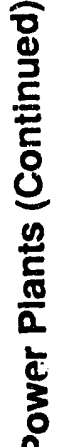

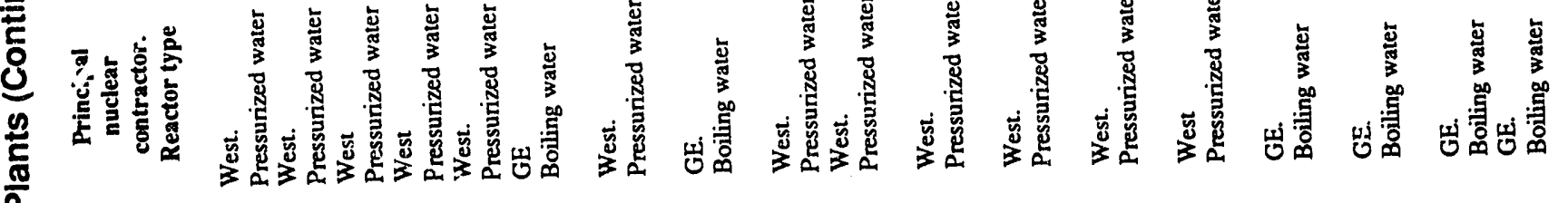

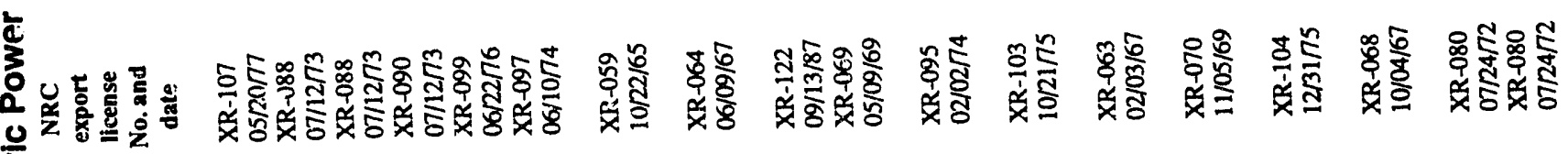

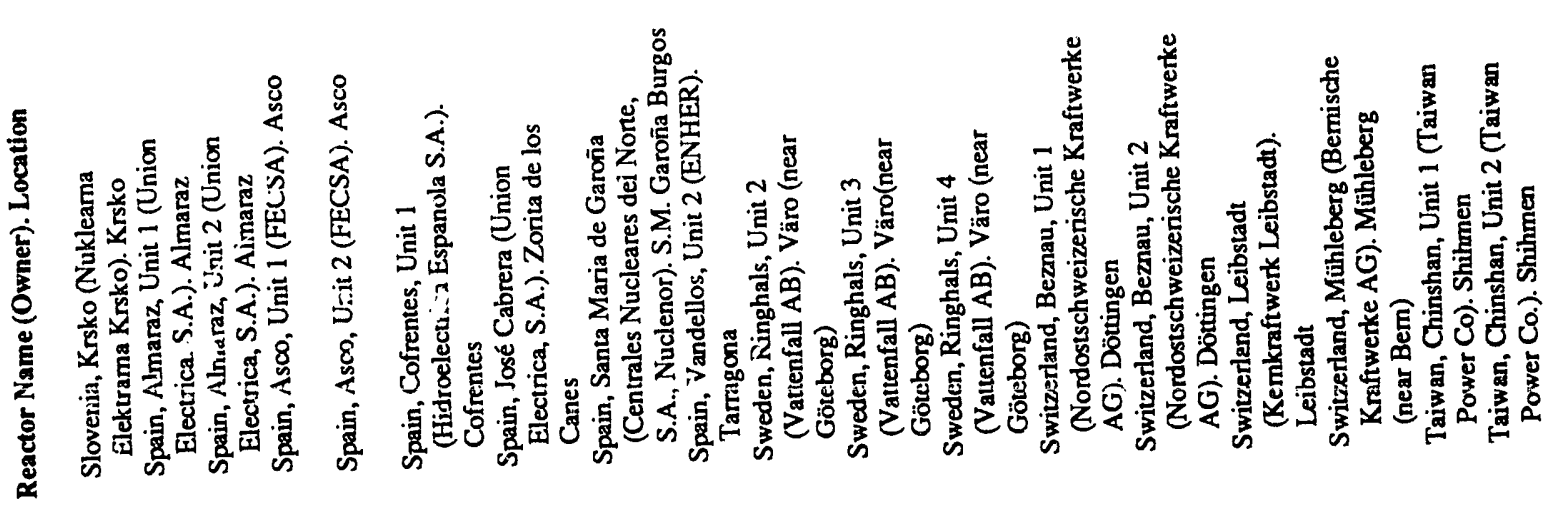




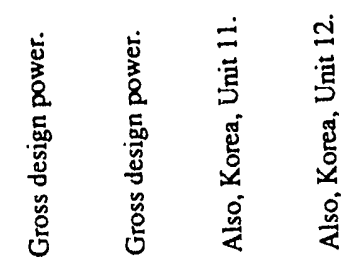

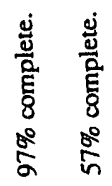

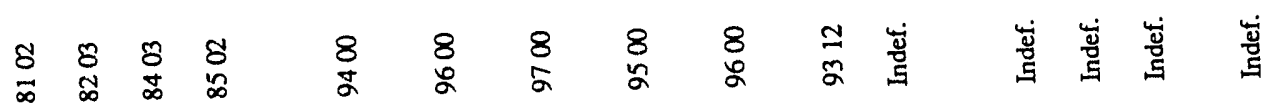

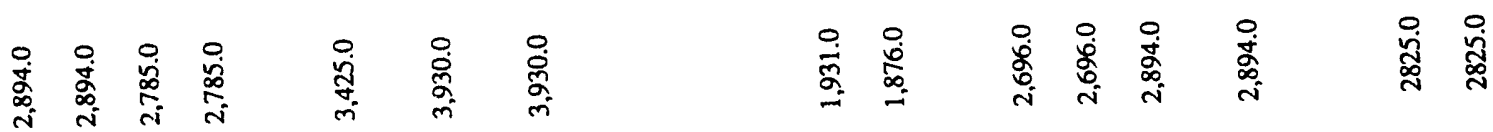

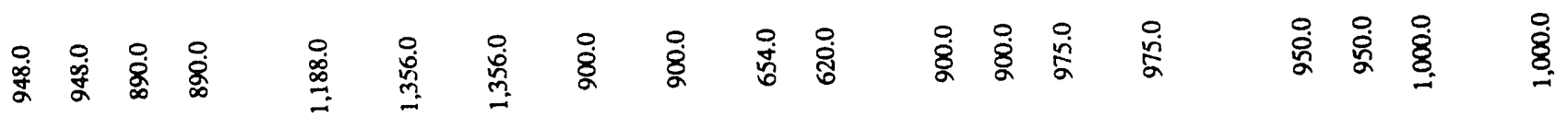

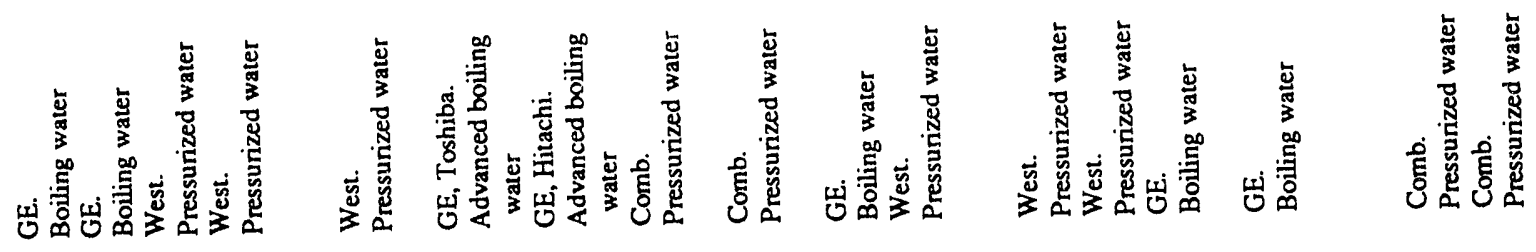

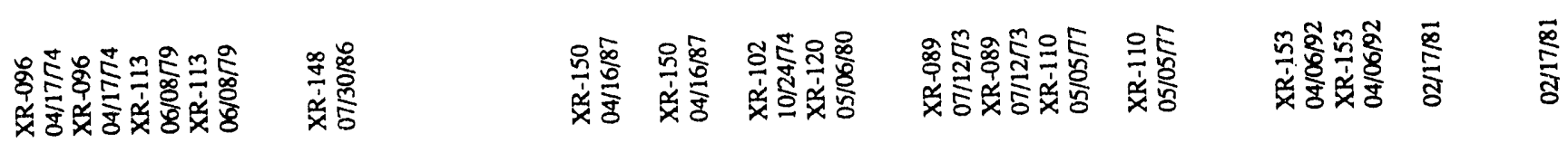<smiles>CC[C@H](C)[C@H](C)CC</smiles>

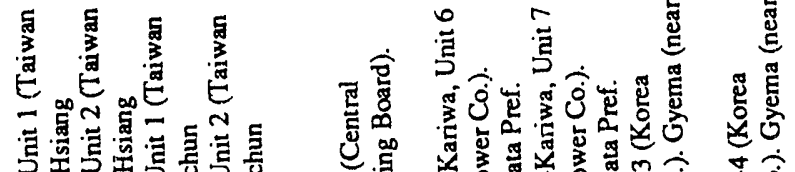

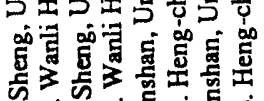

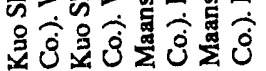
In!min

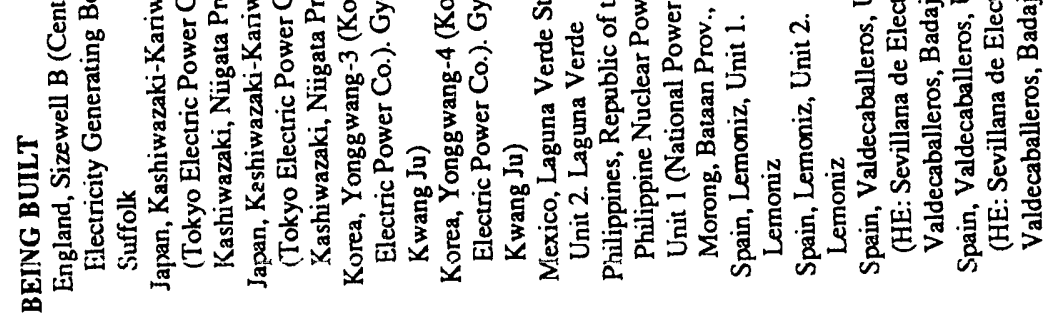

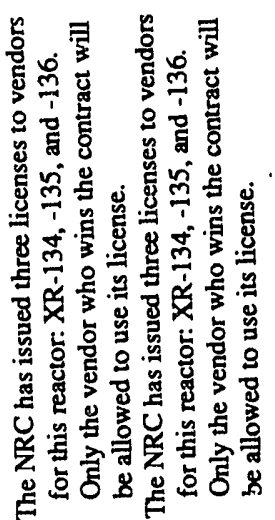

8 


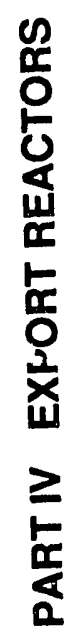

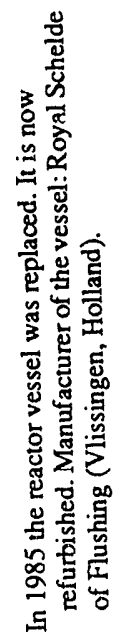

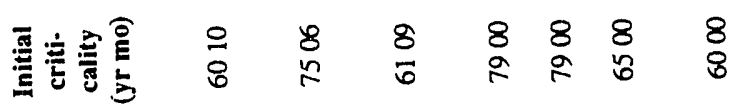

$\frac{8}{6} \frac{8}{8} \frac{8}{\square} \frac{8}{3} \frac{8}{5}$

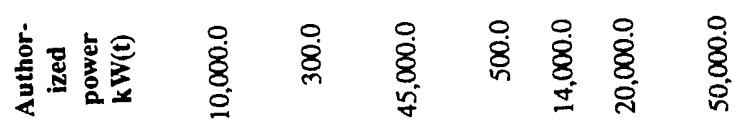

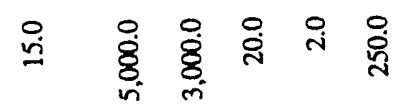

嘿

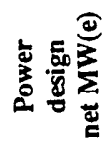

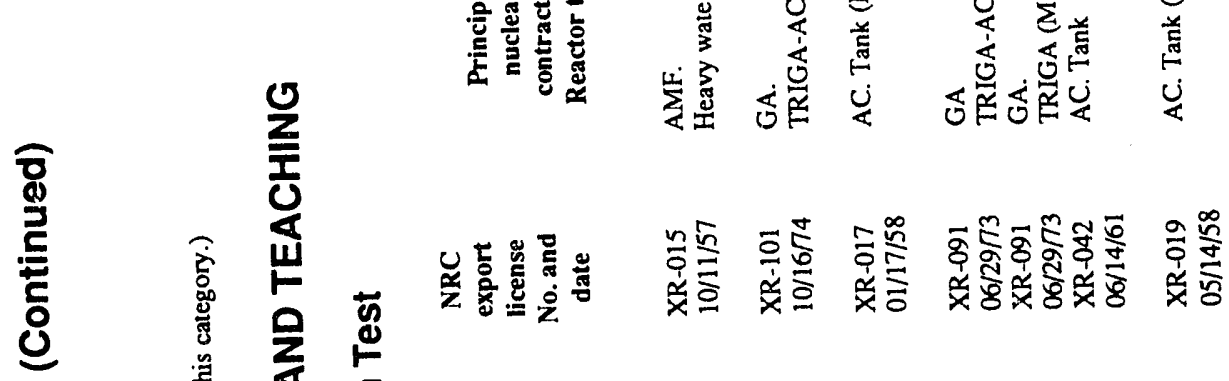

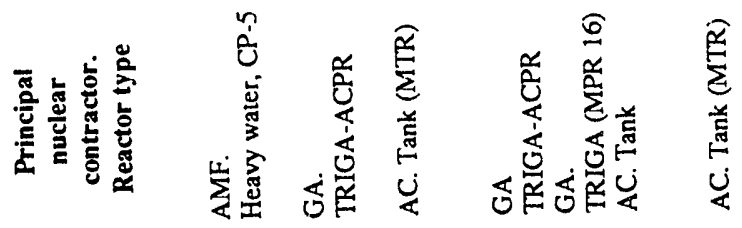

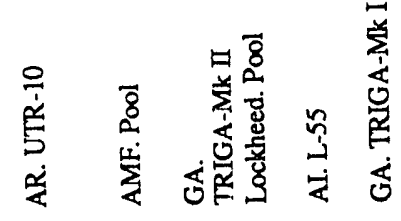

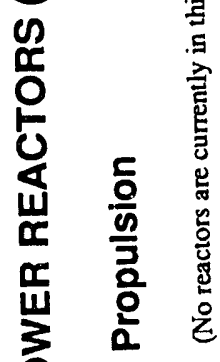

임
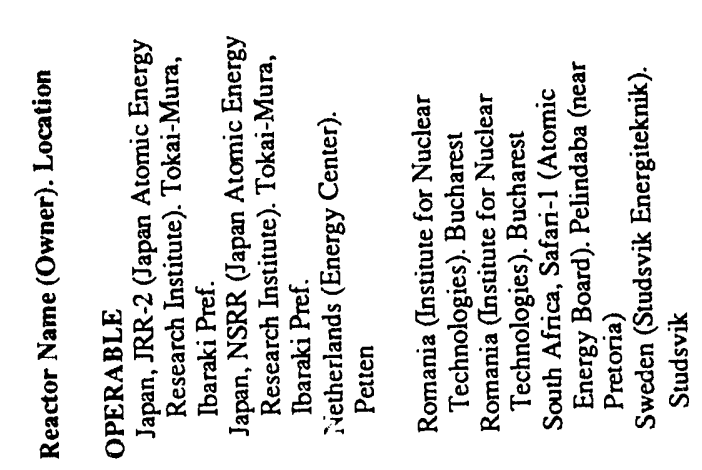

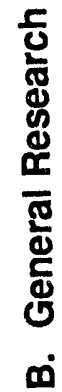

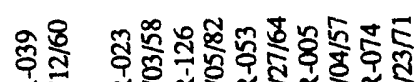

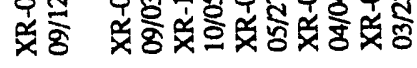

든

市 要

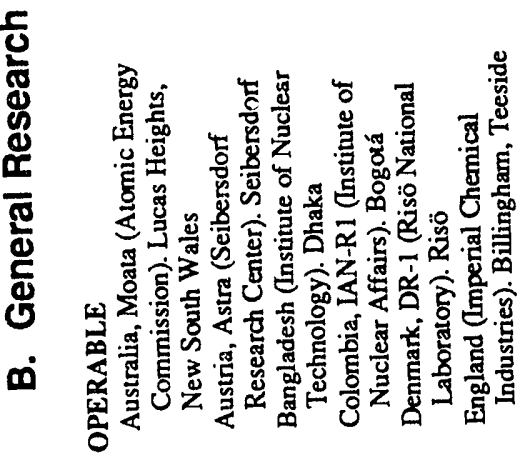



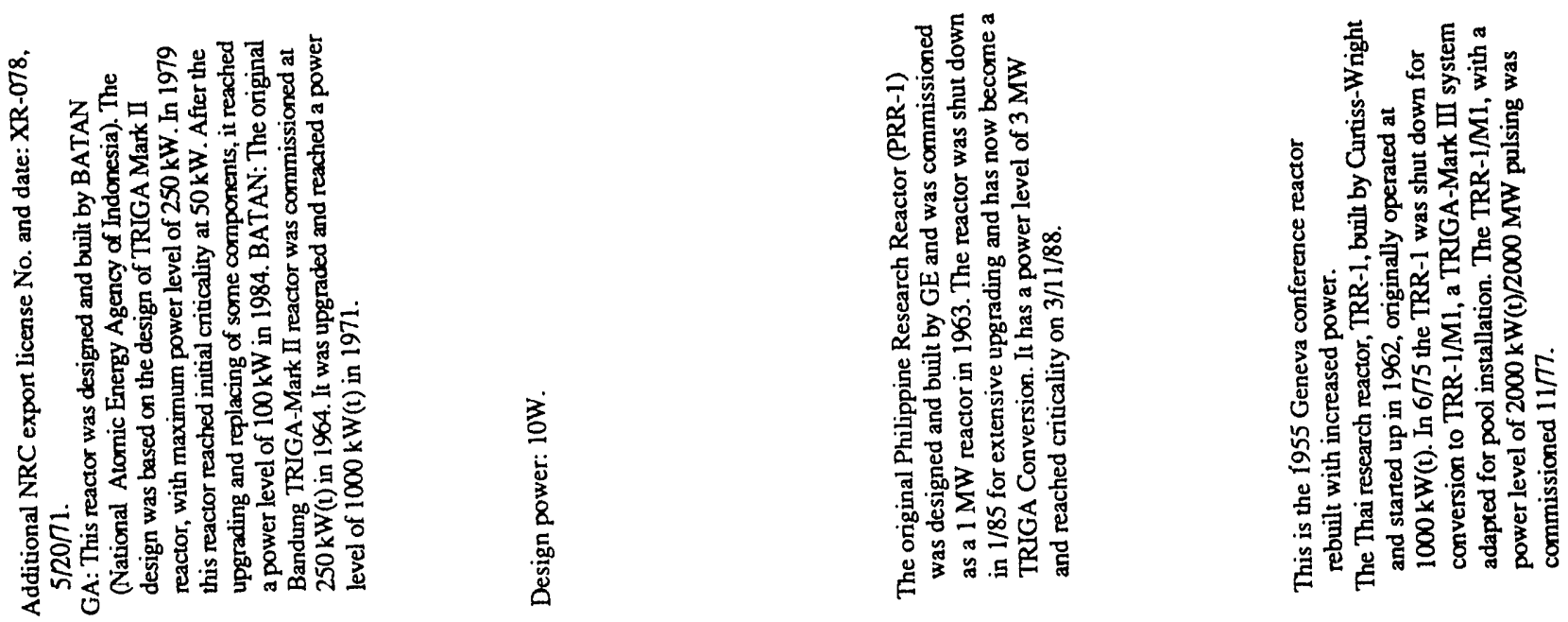

$\frac{8}{8} \frac{8}{8} \frac{8}{8}$

$\frac{8}{8} \frac{8}{8}$

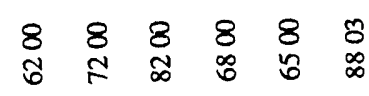

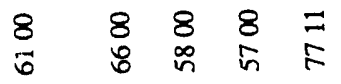

$\begin{array}{ll}8 & 8 \\ 4 & 9\end{array}$

울 울음

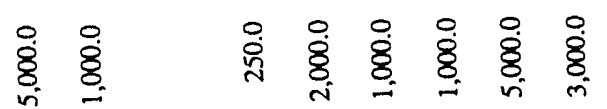

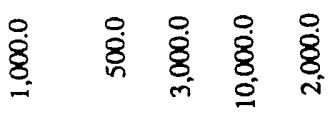

욜

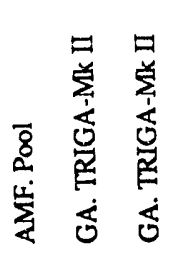

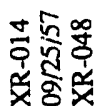

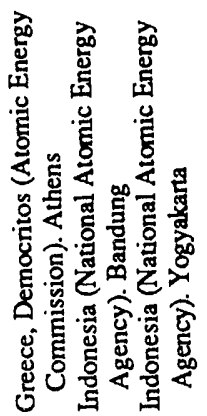

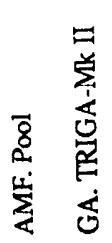

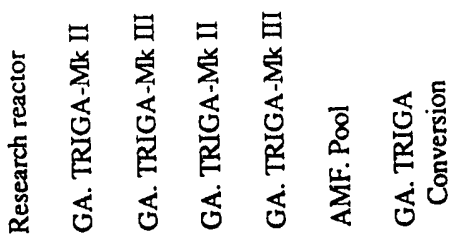

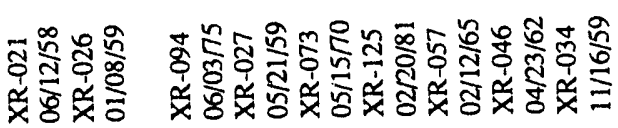

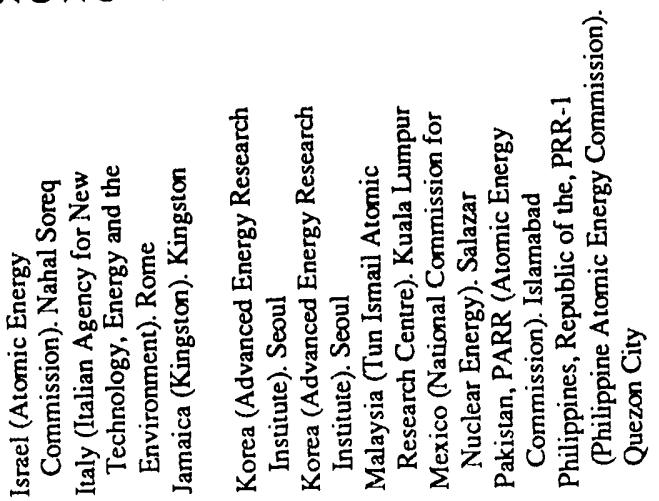

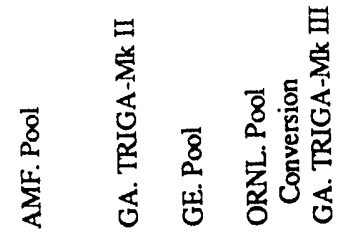

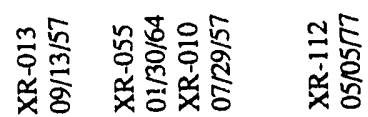

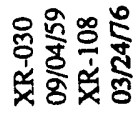
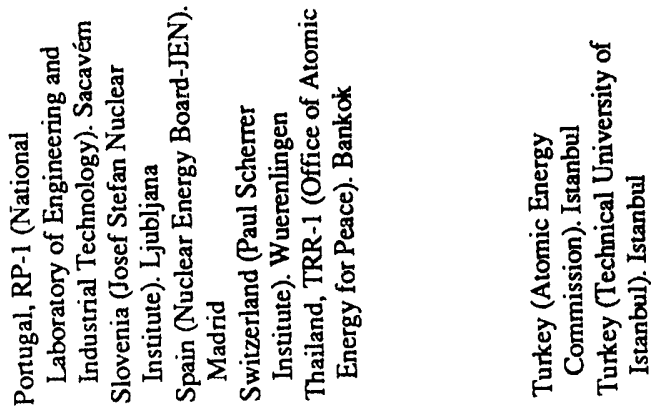


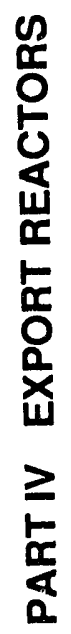
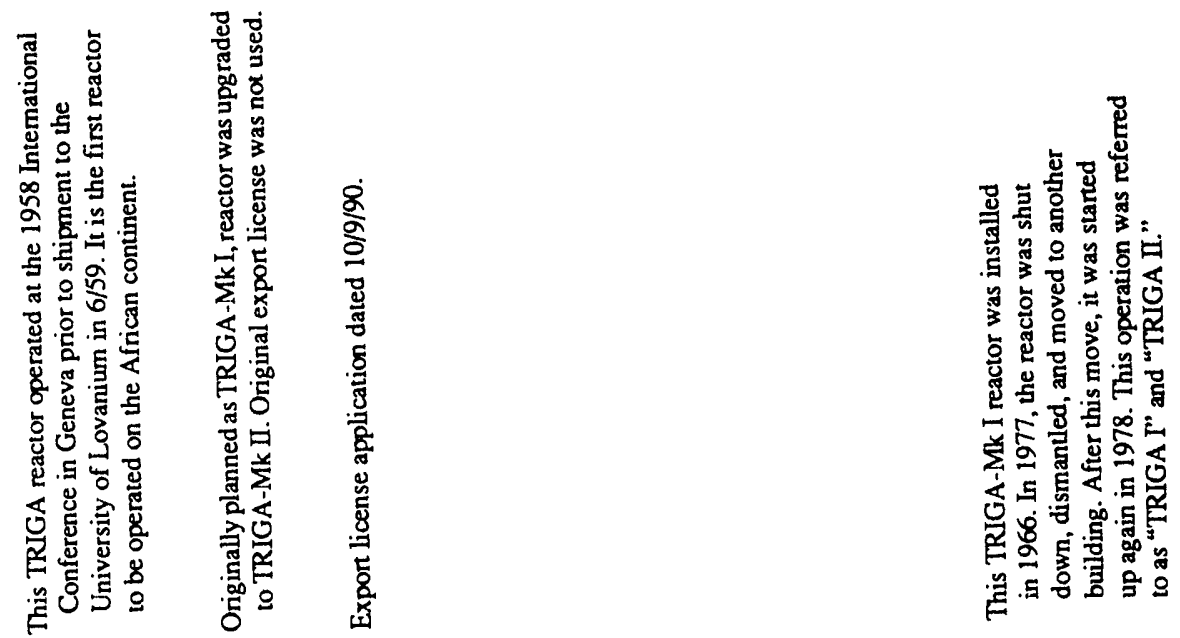

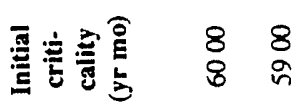

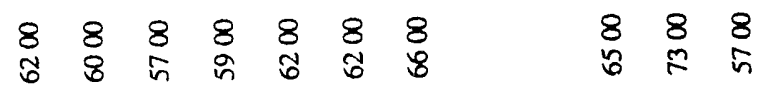

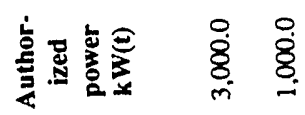

鿷

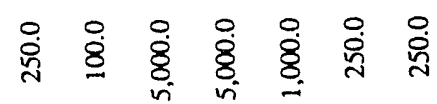

일 옳

嘈

雚

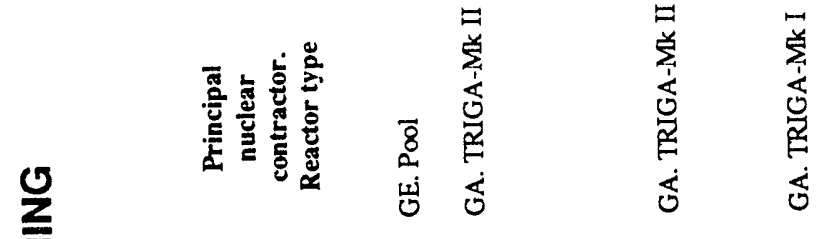

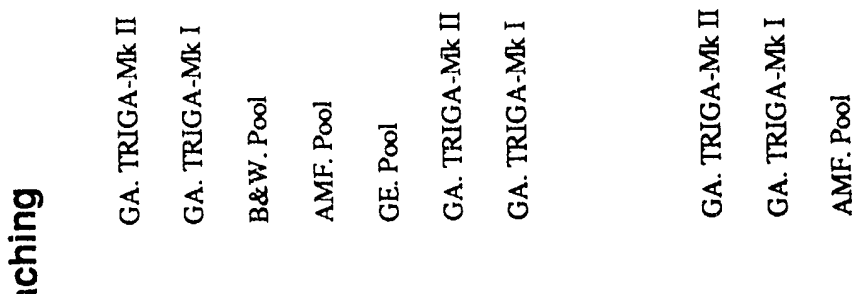

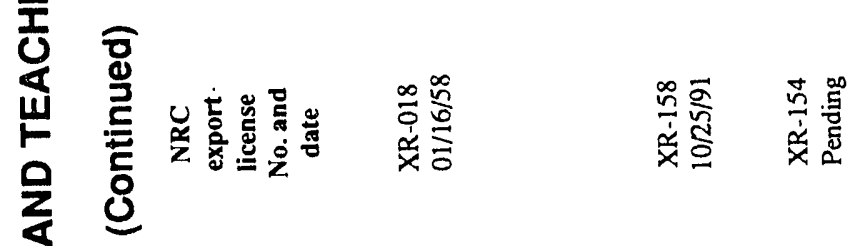

ะ.

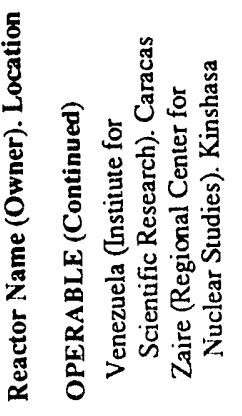

寉

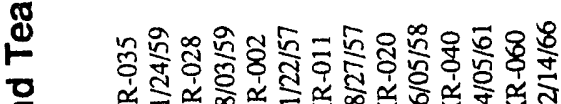

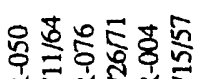

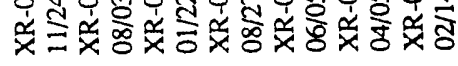

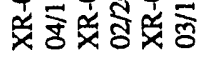

ปั

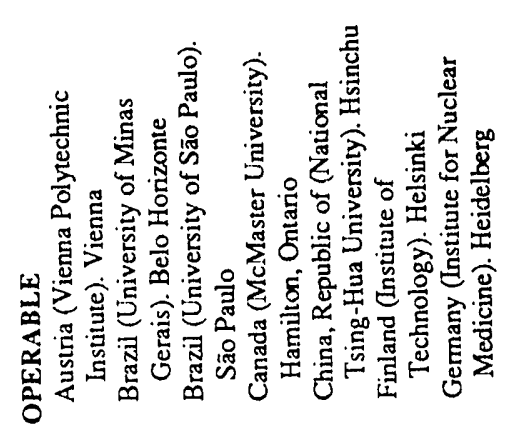

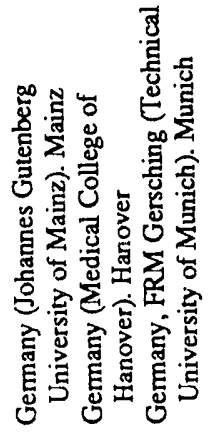




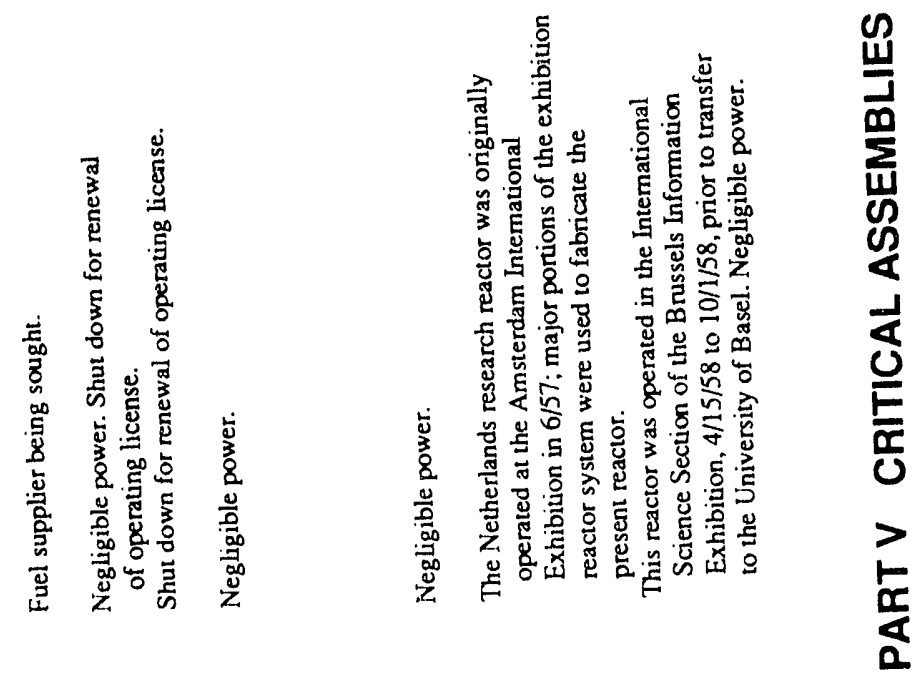

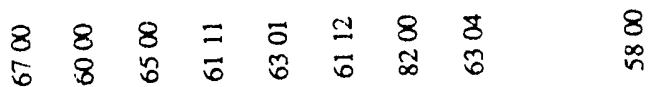

ำ

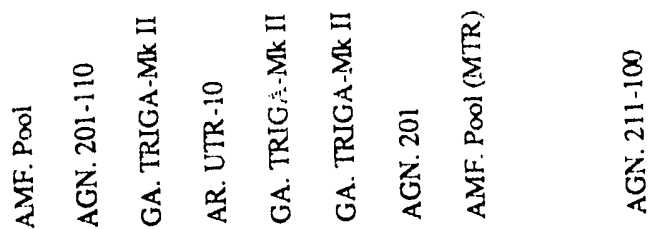

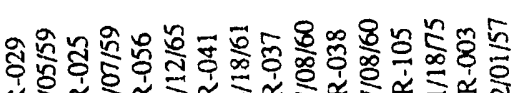

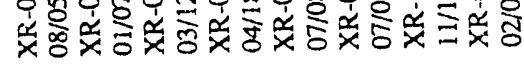

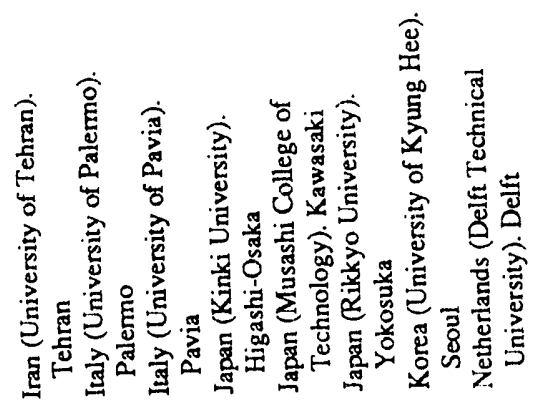

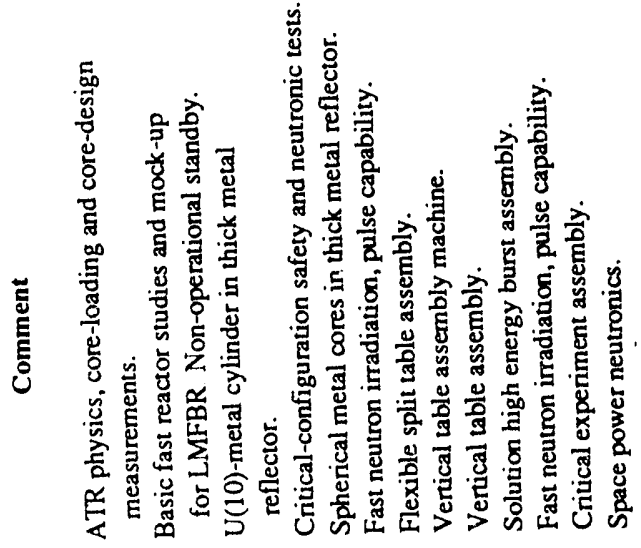

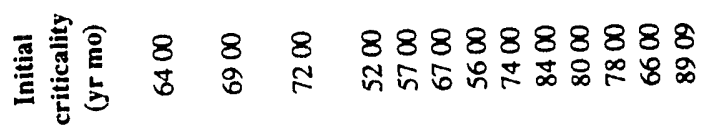

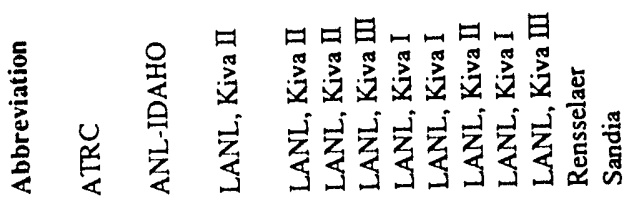

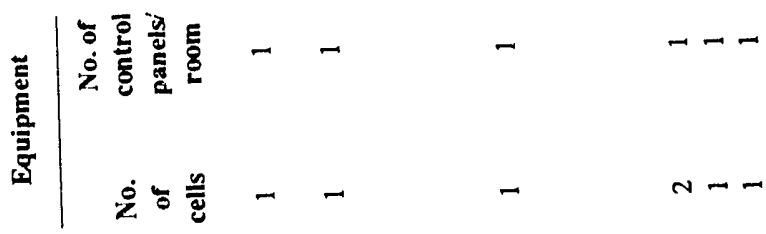

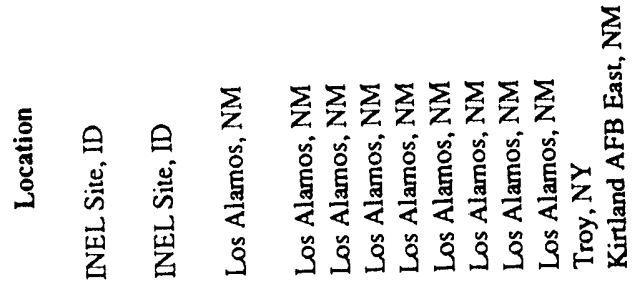

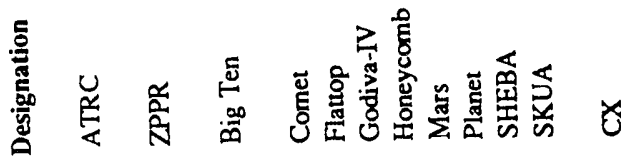

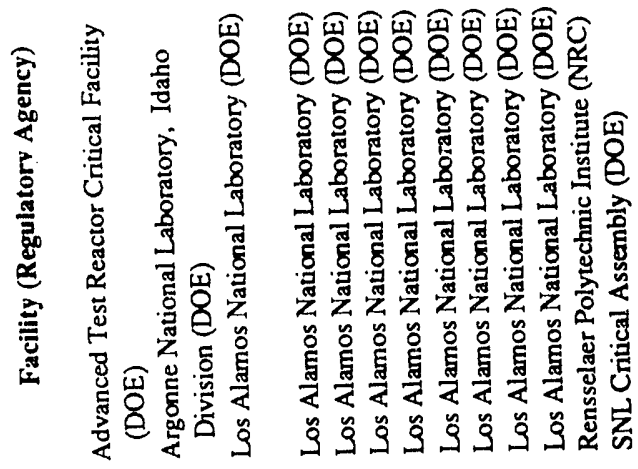




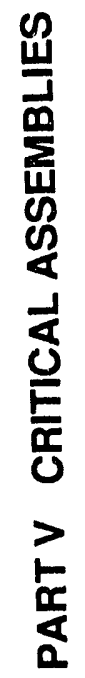

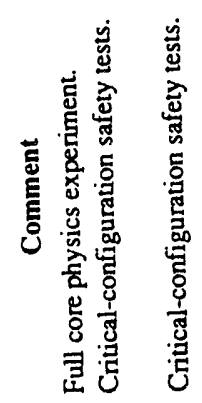

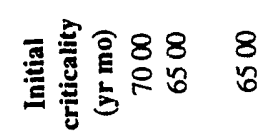

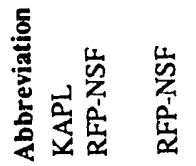

章|

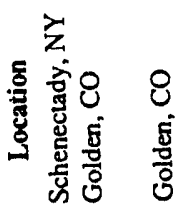

こ

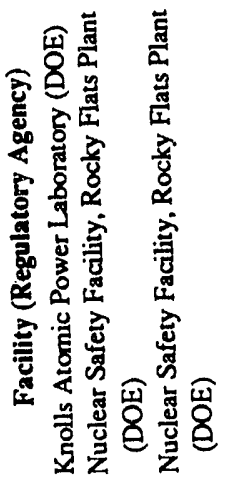




\section{REACTORS AND FACILITIES SHUTDOWN OR DISMANTLED}




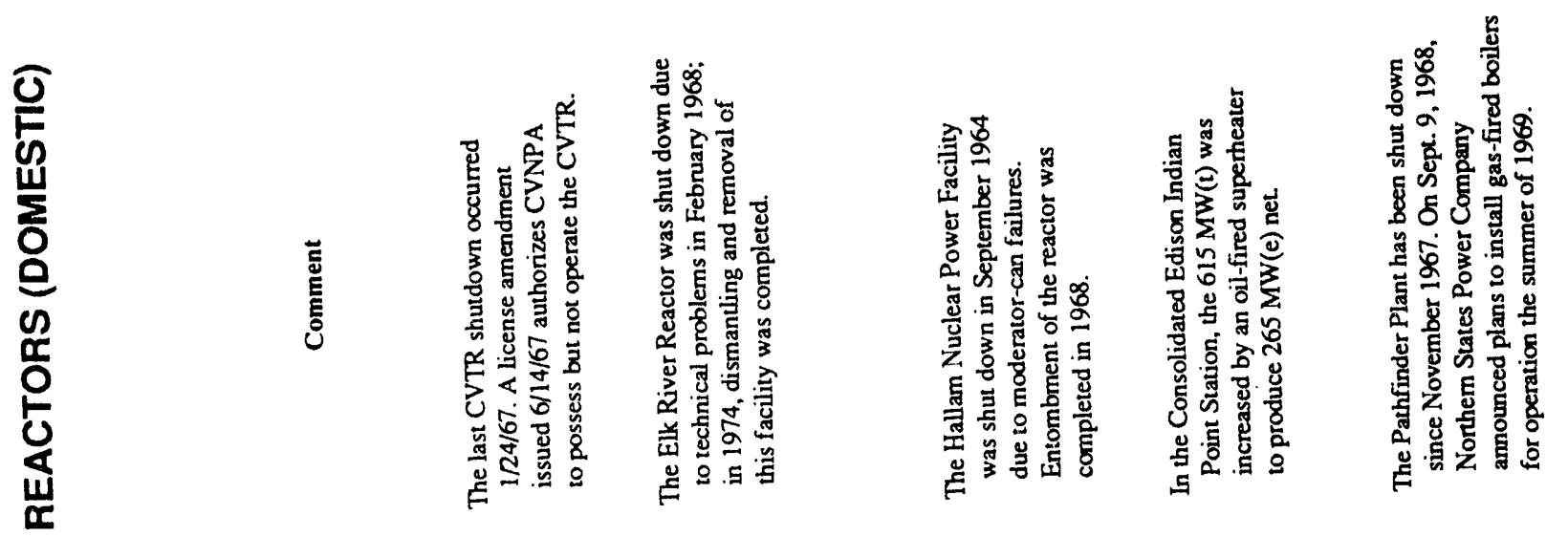

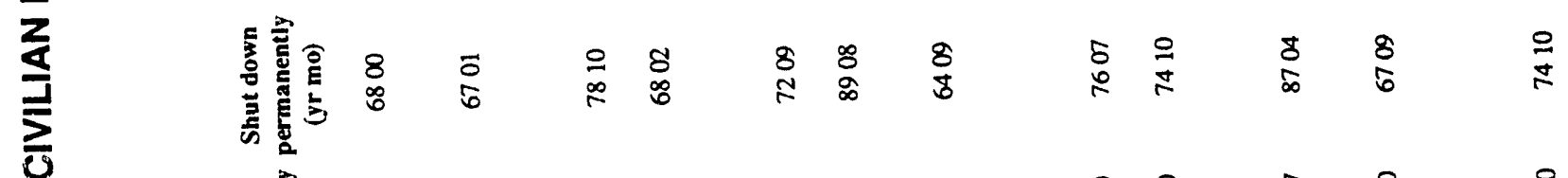

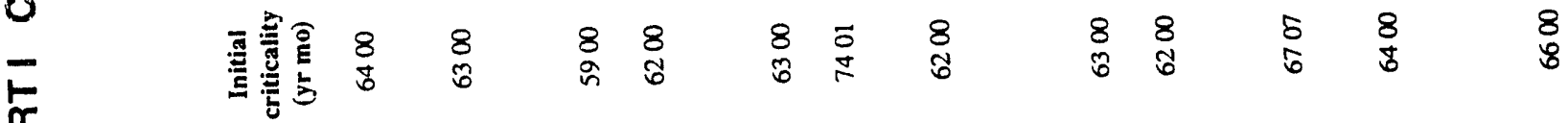

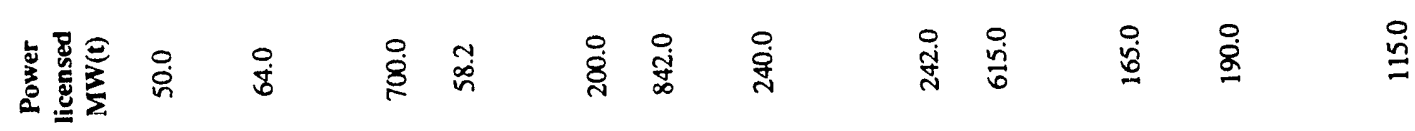

展

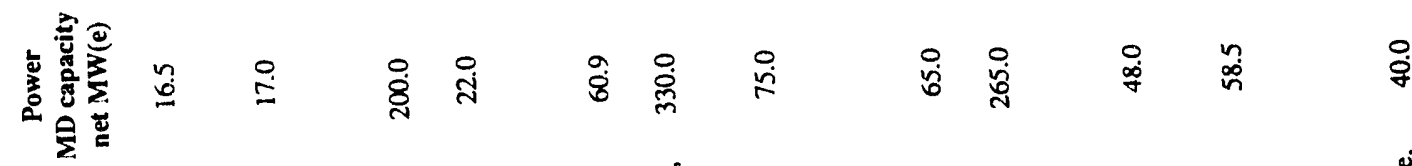

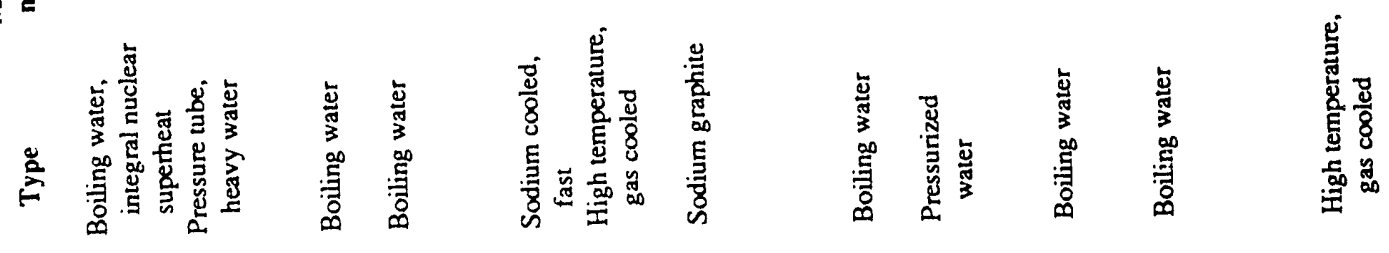

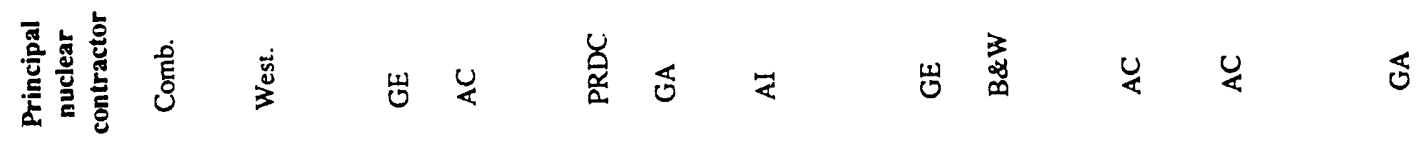

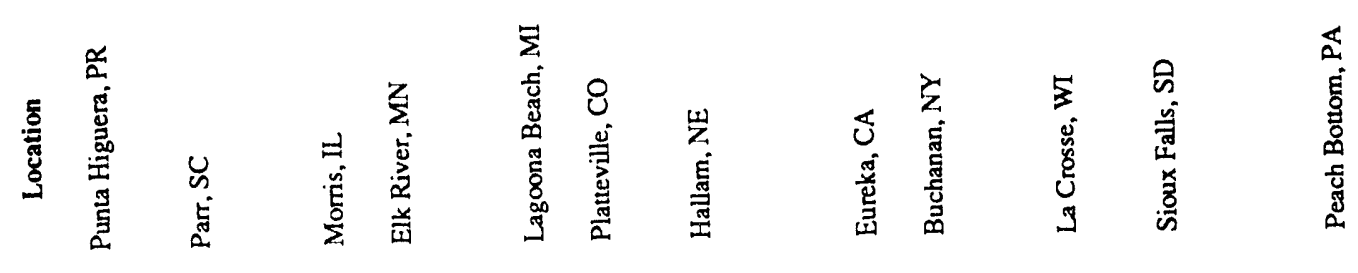

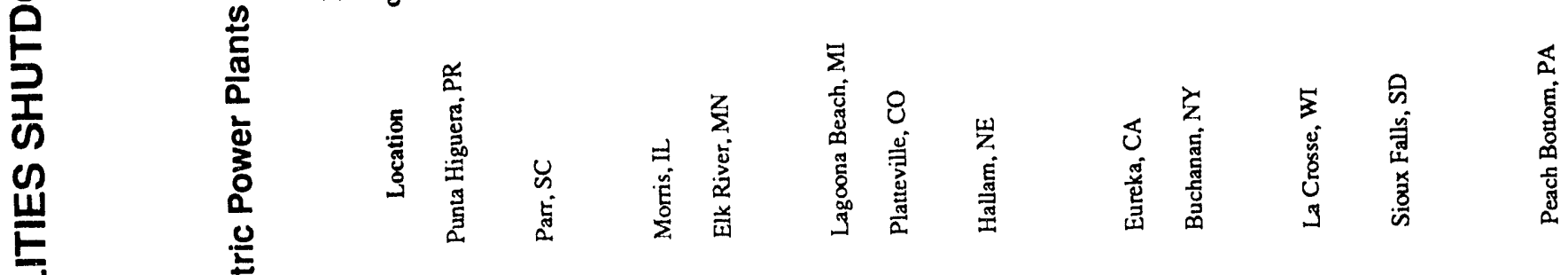

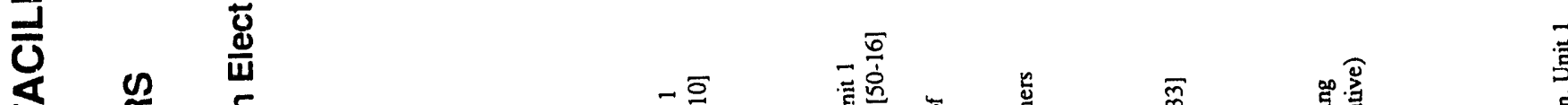

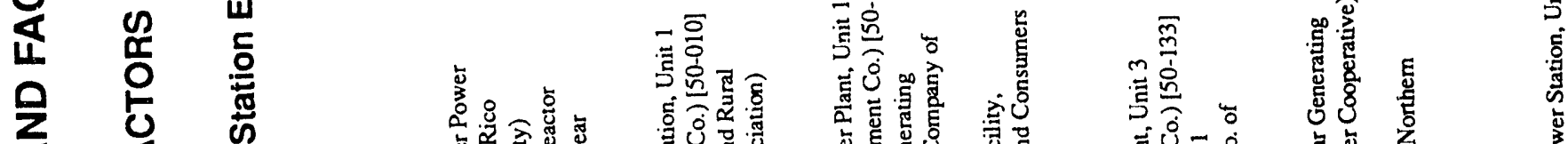

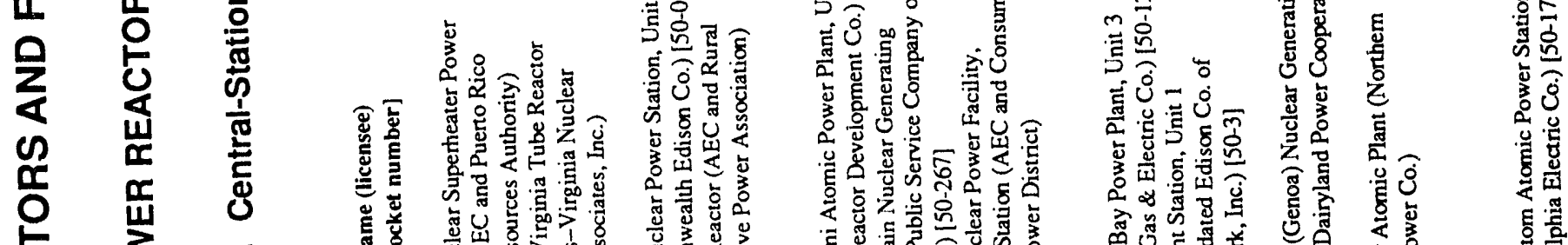

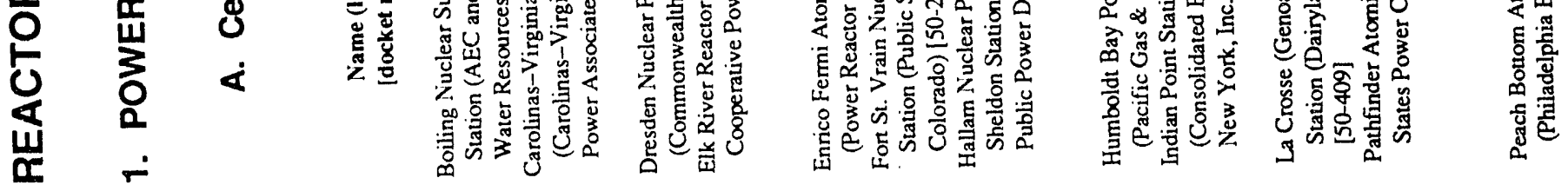




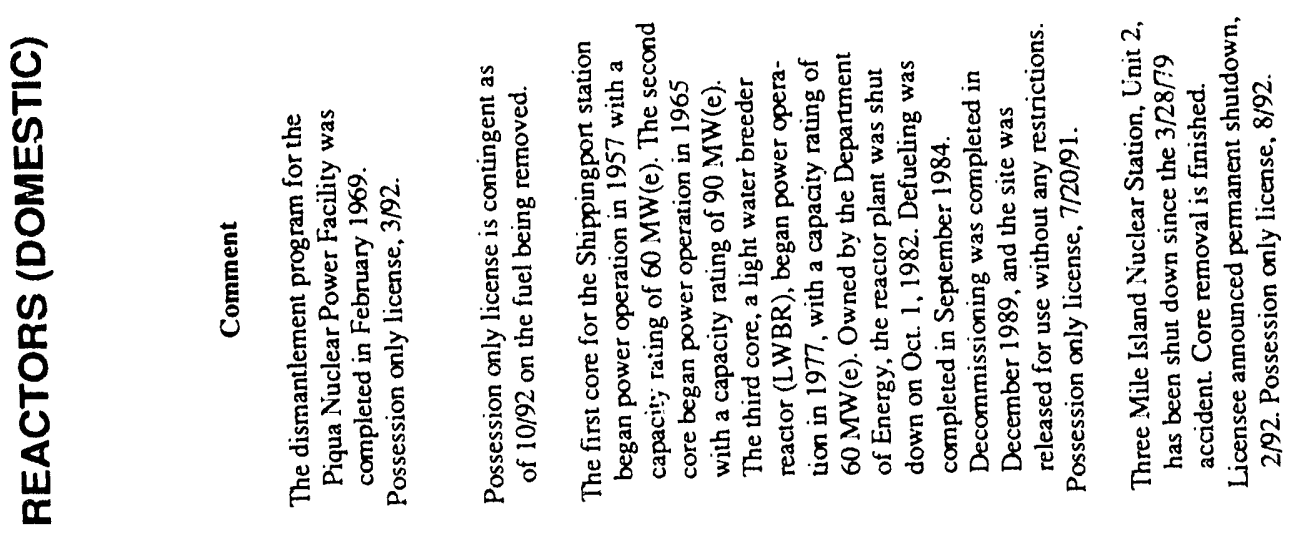

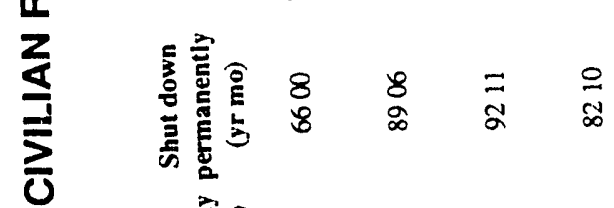

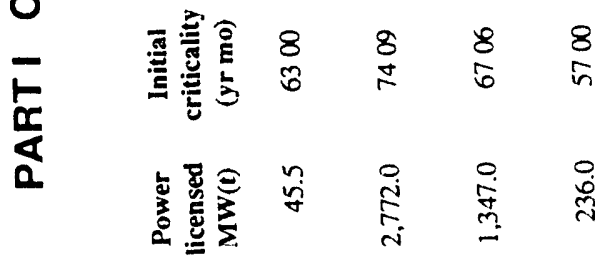

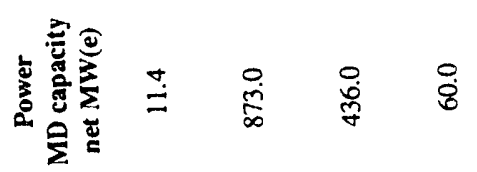

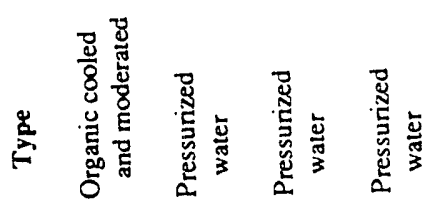

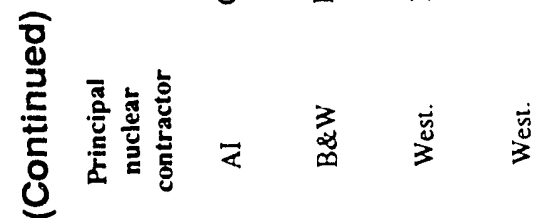

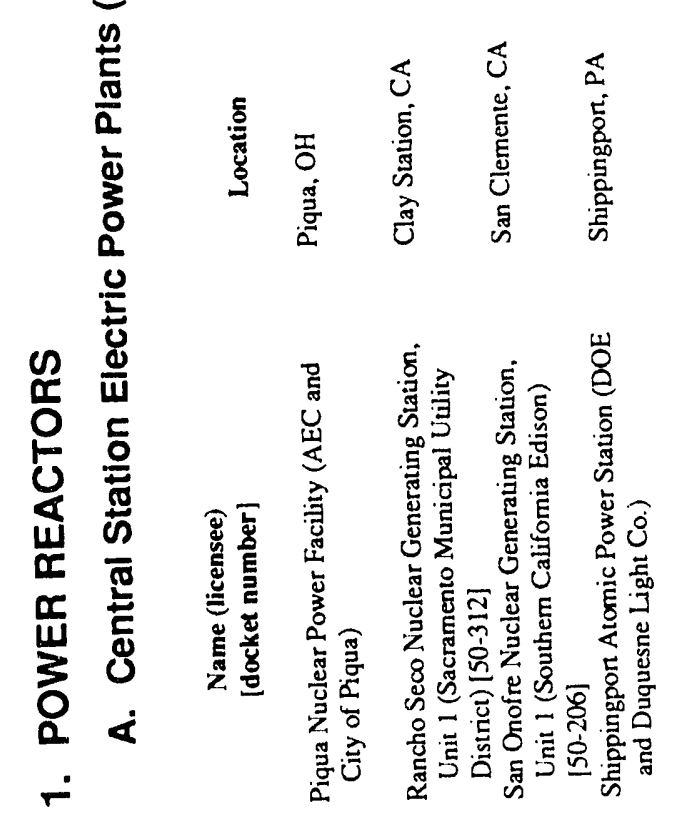

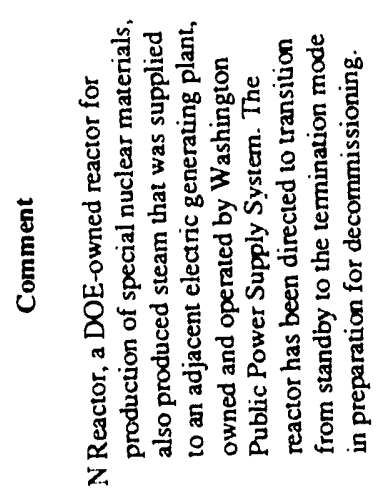

5 8 \%

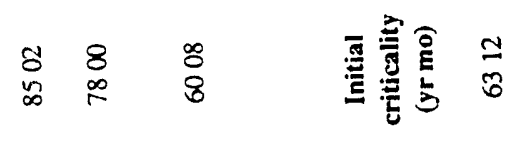

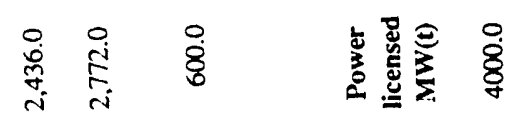

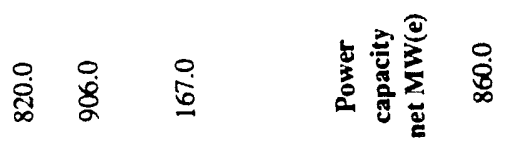

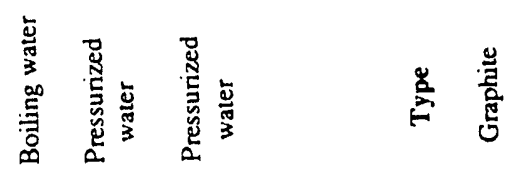

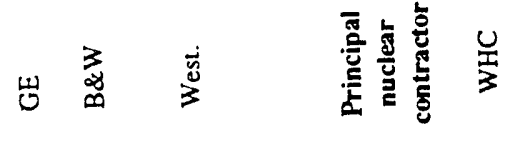

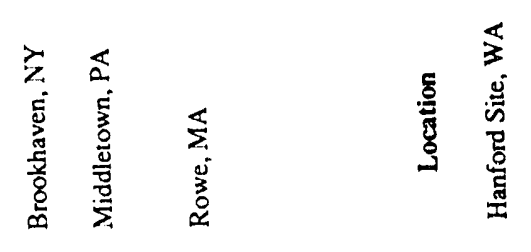

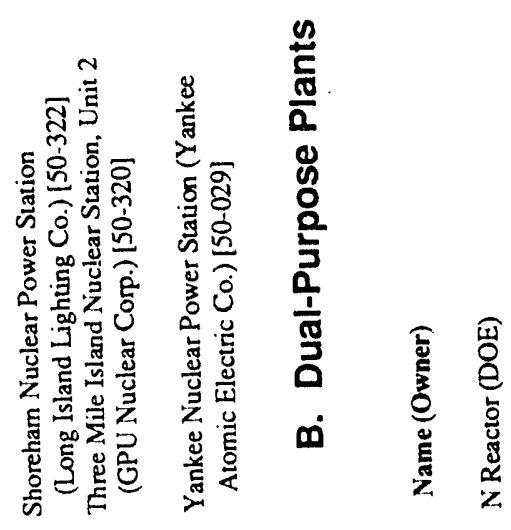




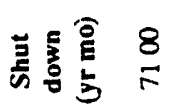

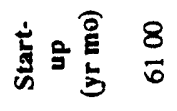

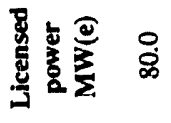

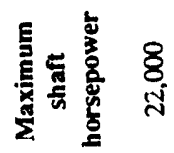

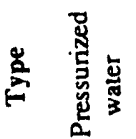

竎

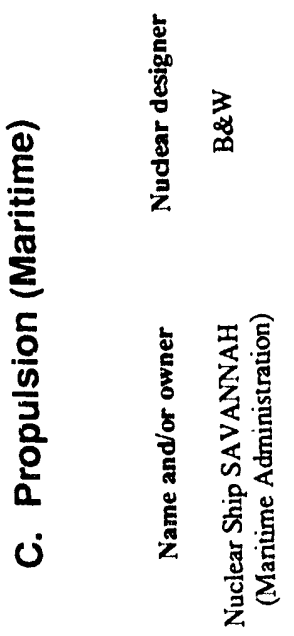

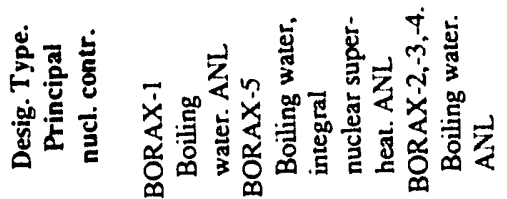

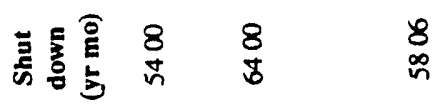

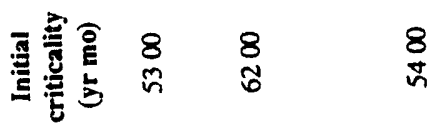

害蜜

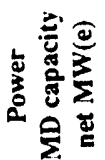

僖旁言

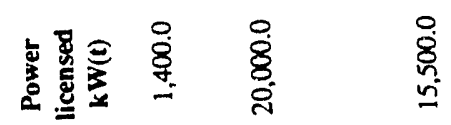

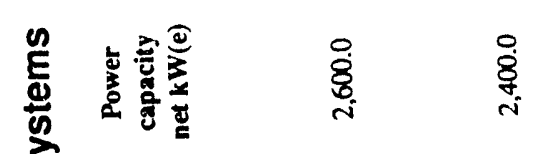

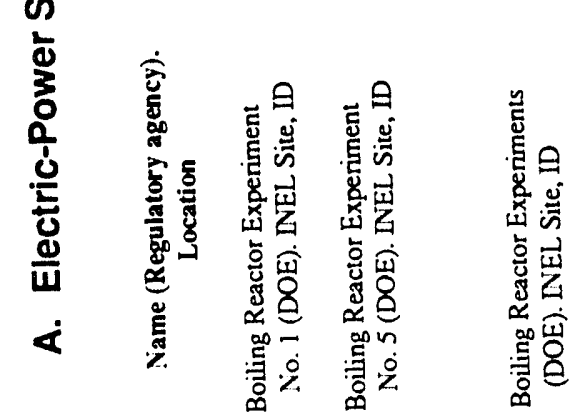

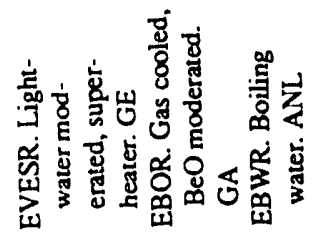

$\begin{array}{ll}8 & 8 \\ 5 & 5\end{array}$

$\begin{array}{ll}8 & 8 \\ 1 & 8\end{array}$

憘耪

ริ)

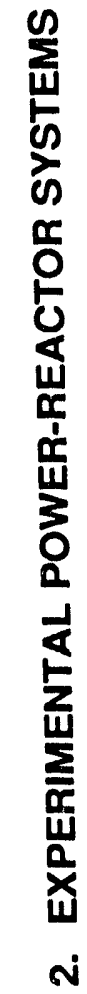



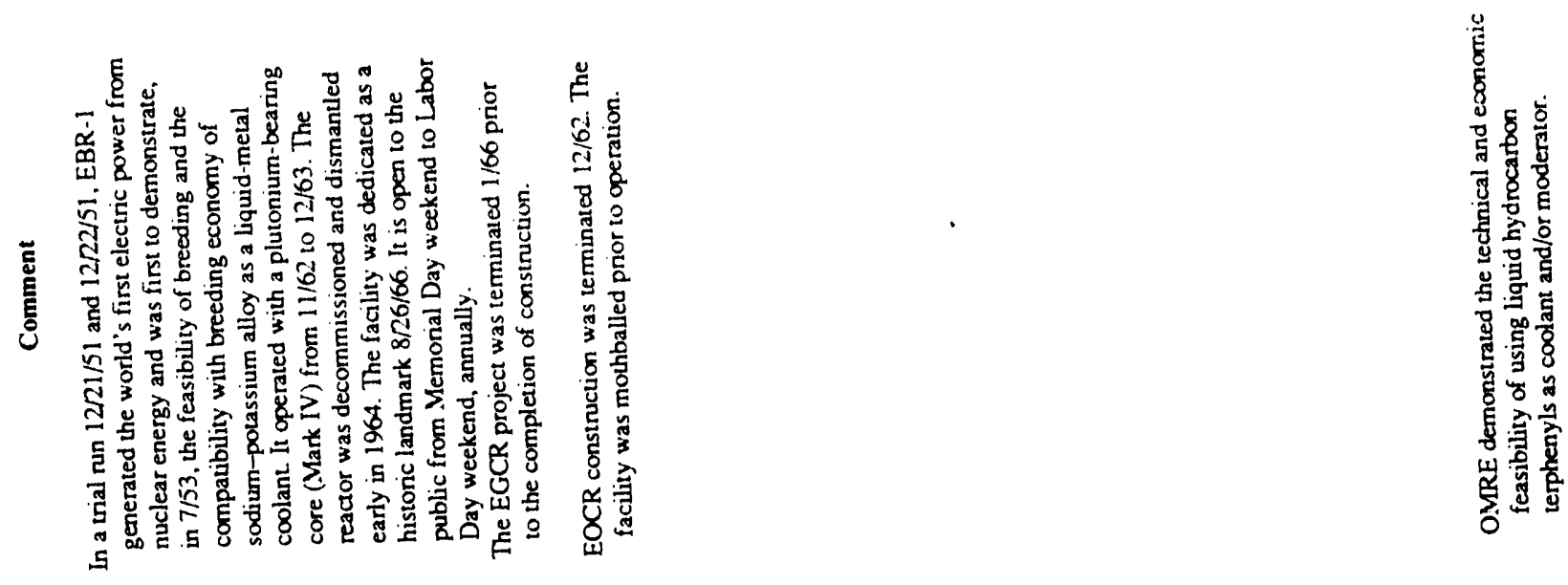

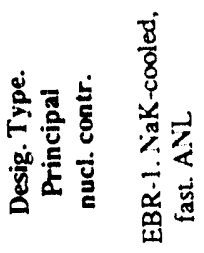

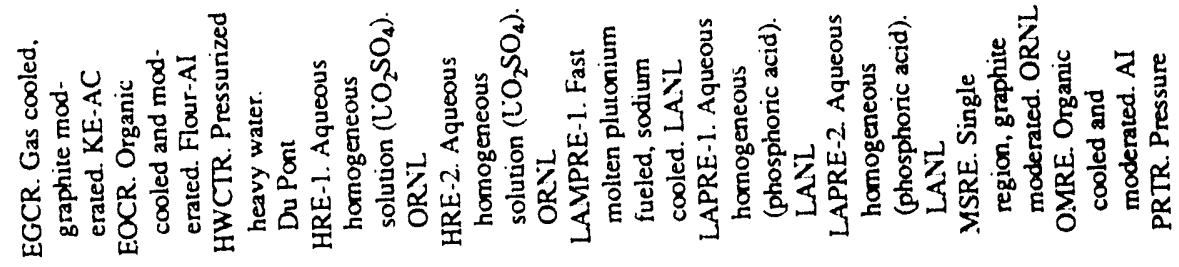

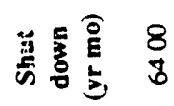

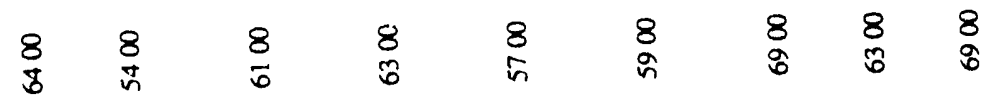

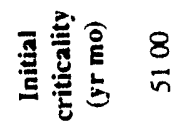

$\begin{array}{lllllllll}8 & 8 & 8 & 8 & 8 & 8 & 8 & 8 & 8 \\ i & i & i & 6 & n & 0 & 0 & 5 & 8\end{array}$

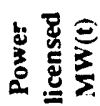

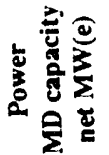

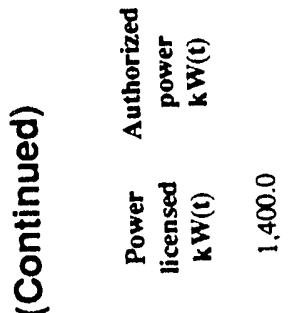

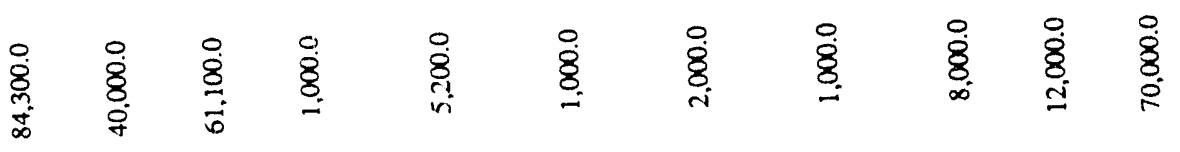

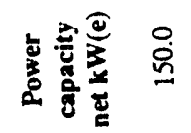

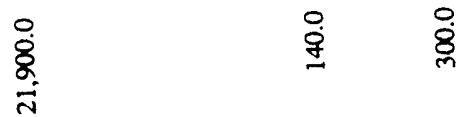

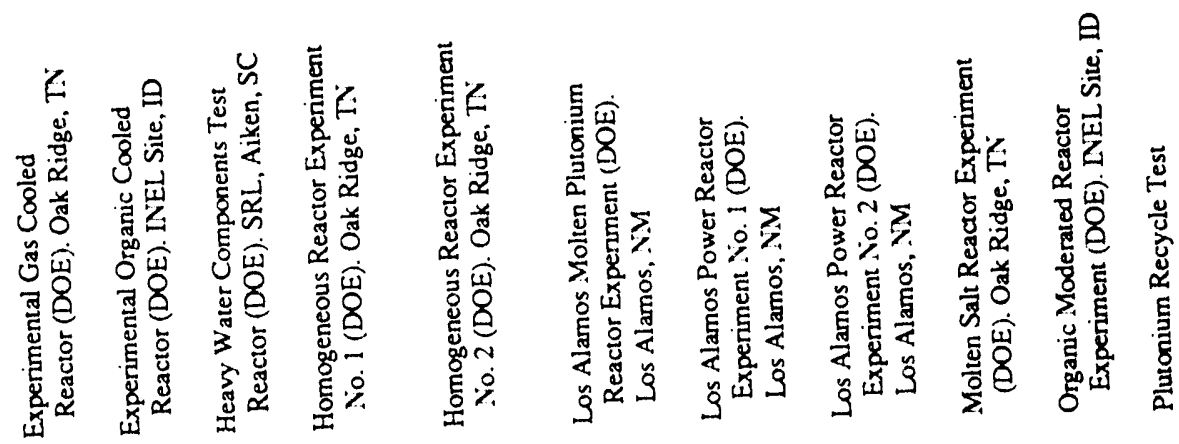




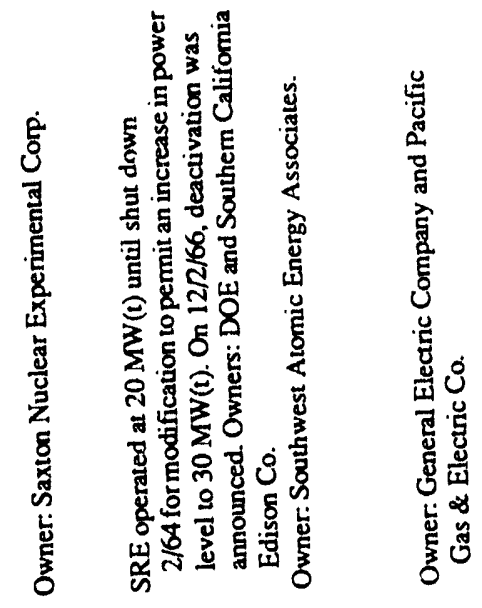

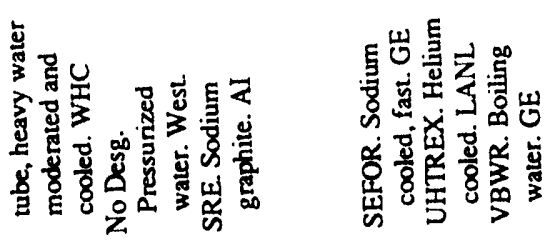

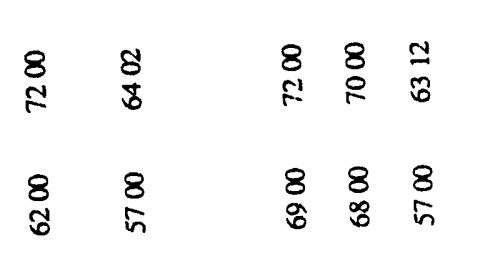

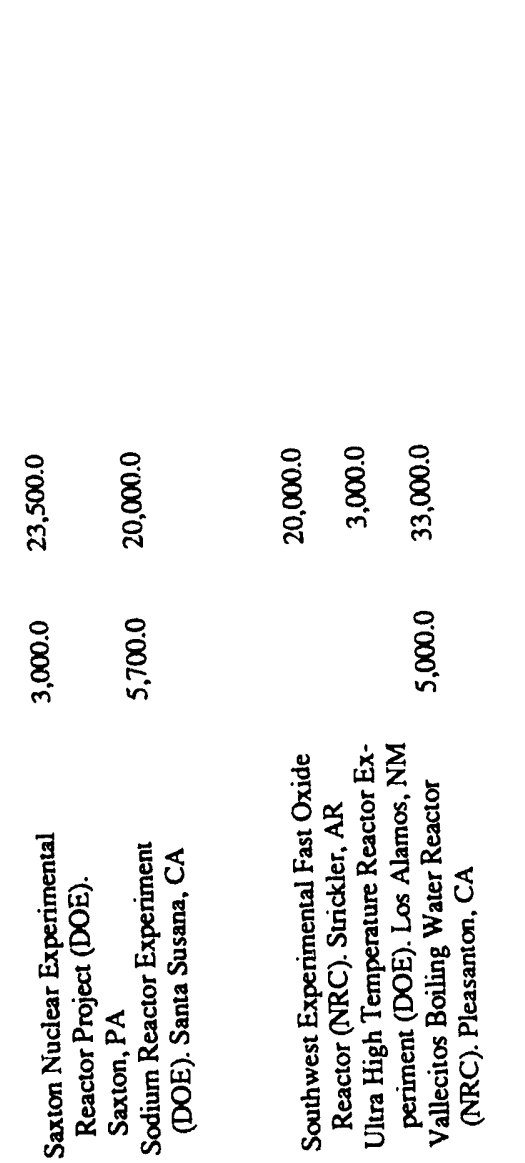

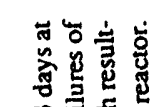
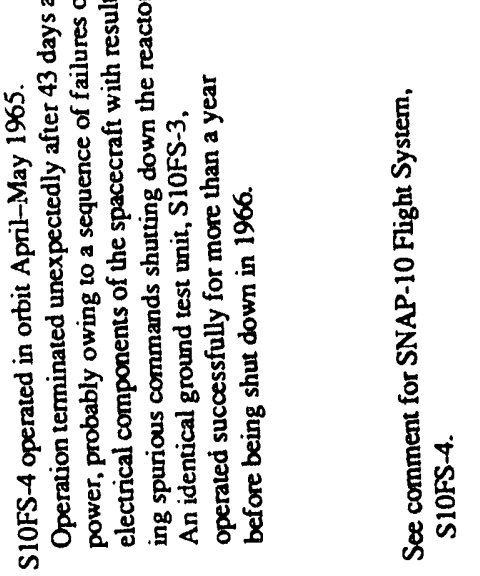

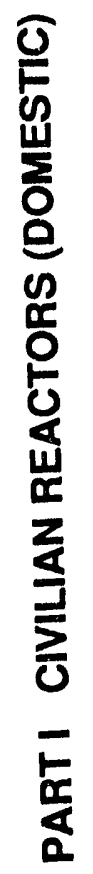
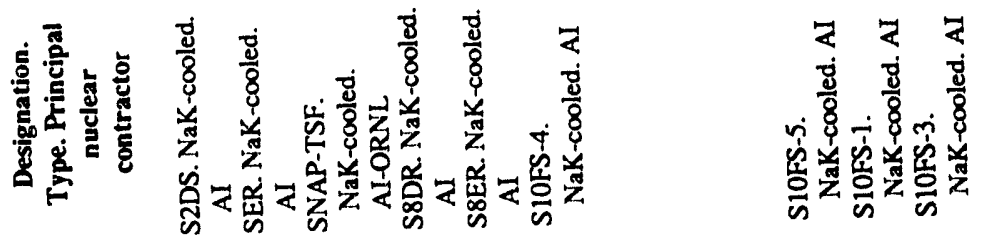

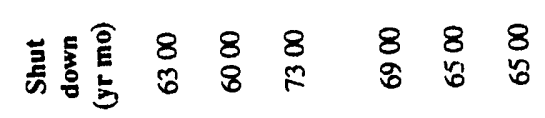

$\begin{array}{ll}8 & 8 \\ 5 & 8\end{array}$

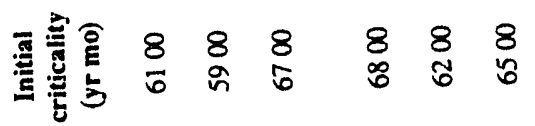

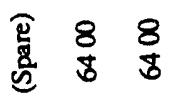

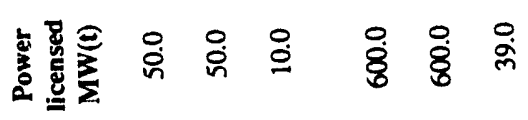

융 잉

焉总

กั กั

递产

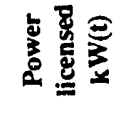

n

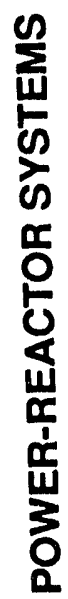

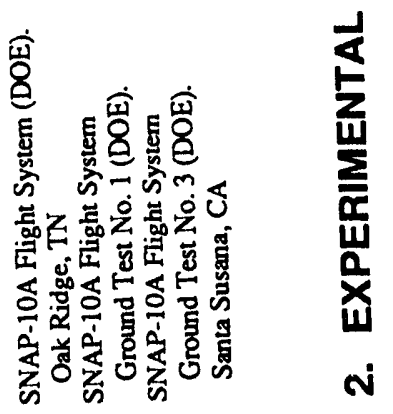


离

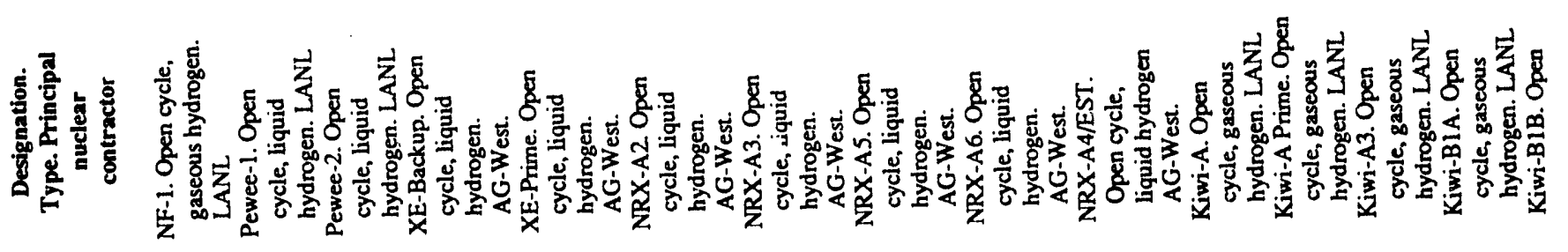

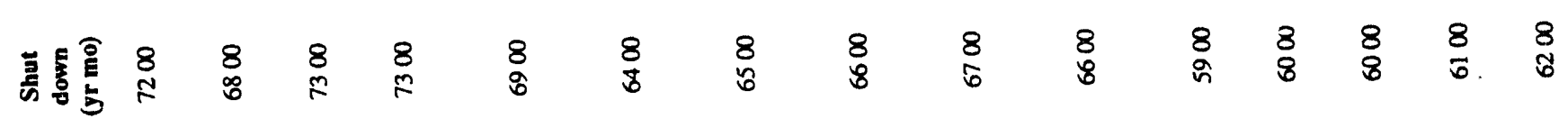

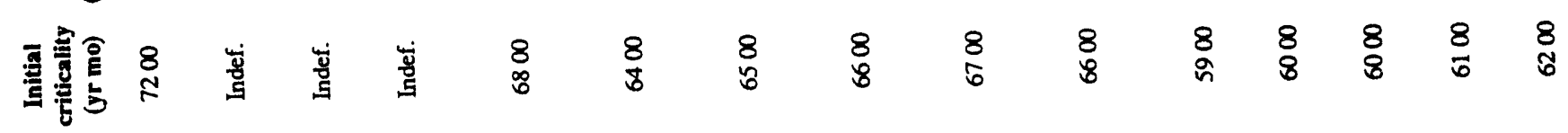

In

䧚

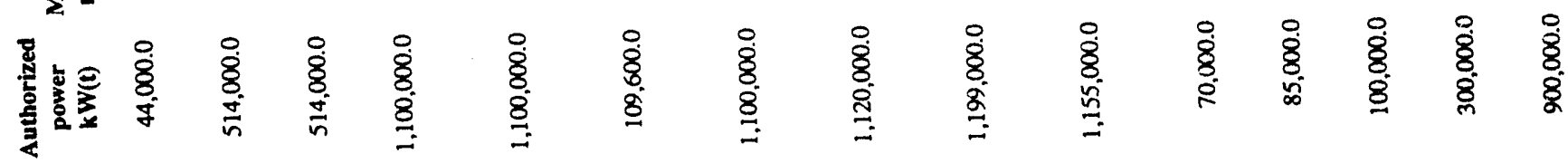

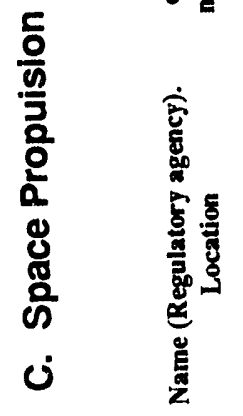

ᄃ

me 


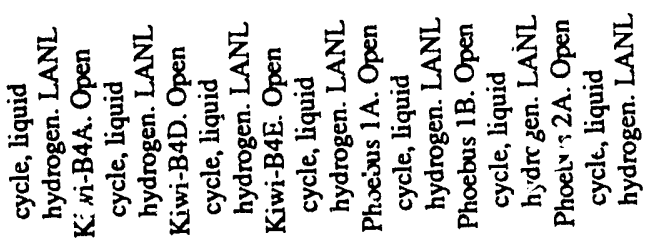

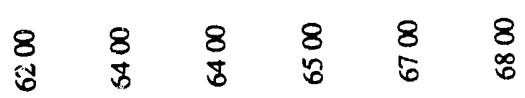

\begin{tabular}{llllll}
8 & 8 & 8 & 8 & 8 & 8 \\
\hline & 8 & 8 & 8
\end{tabular}

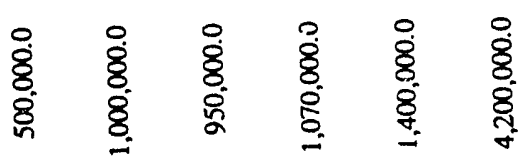

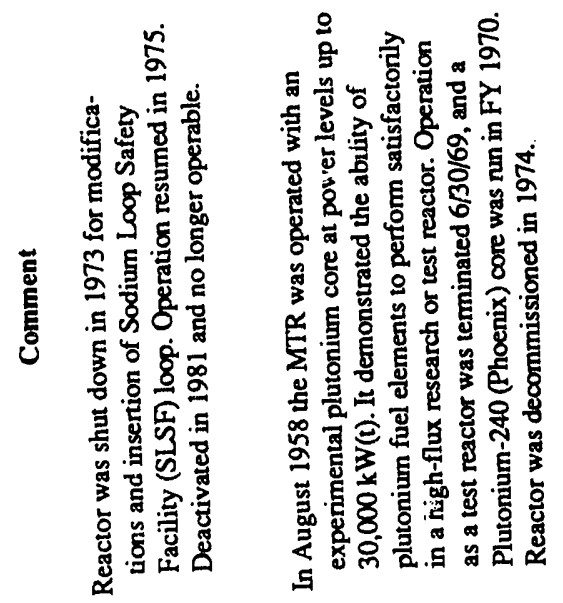

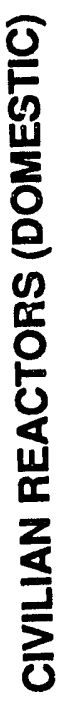

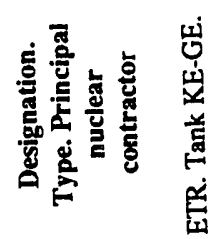
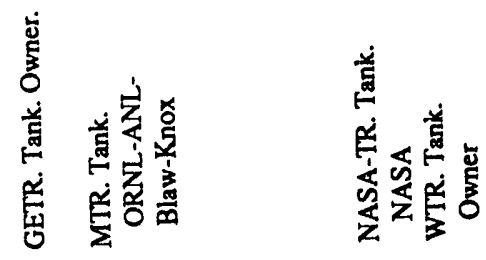

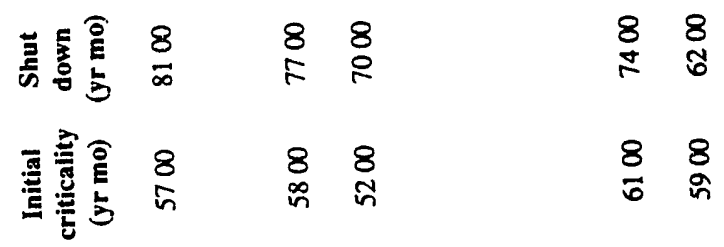

密蜜

采

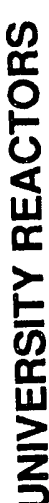

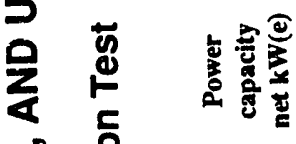

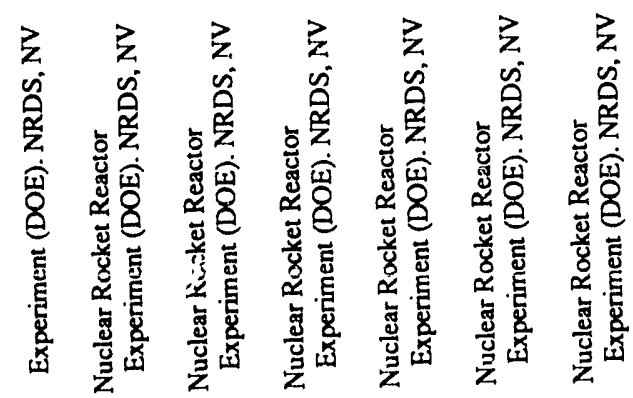

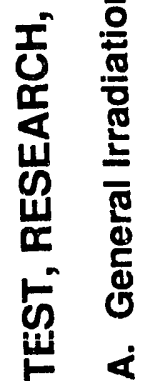

m
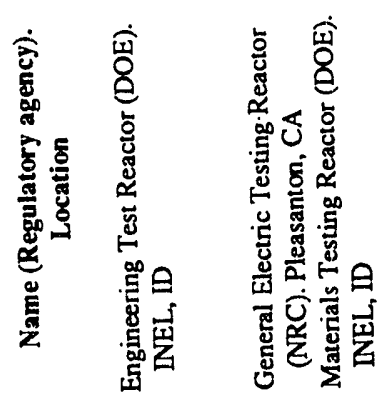

$\begin{array}{ll}0 & 0 \\ 8 & 8 \\ 8 & 8 \\ 8 & 8\end{array}$

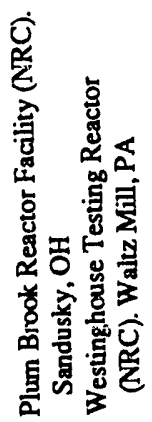

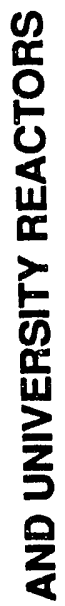

壱 mं 


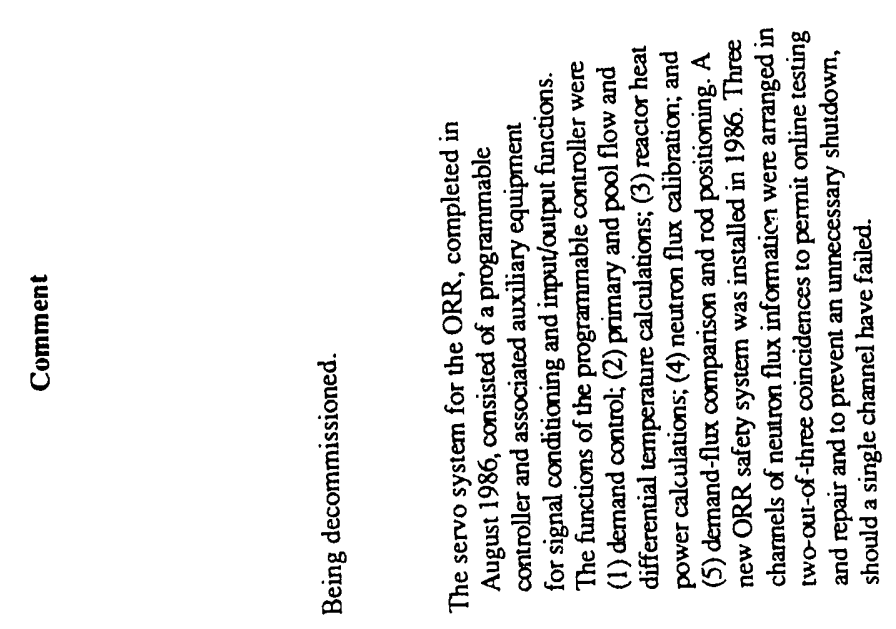

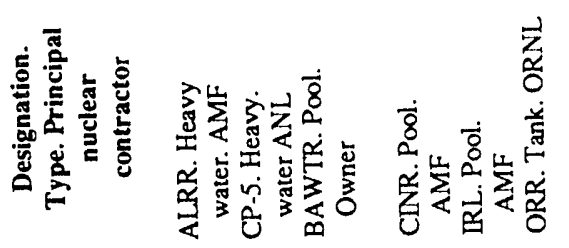

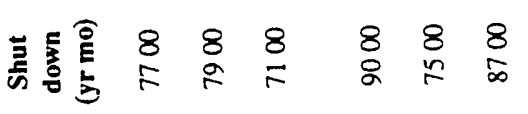

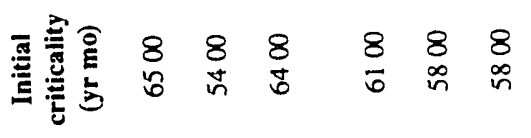

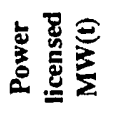

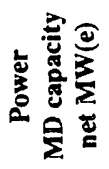

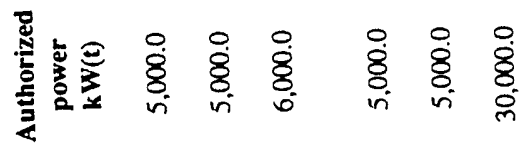

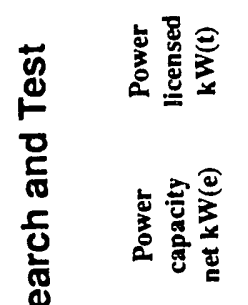

妾

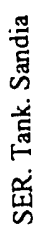

8

$\frac{8}{6}$

0
8
8
n

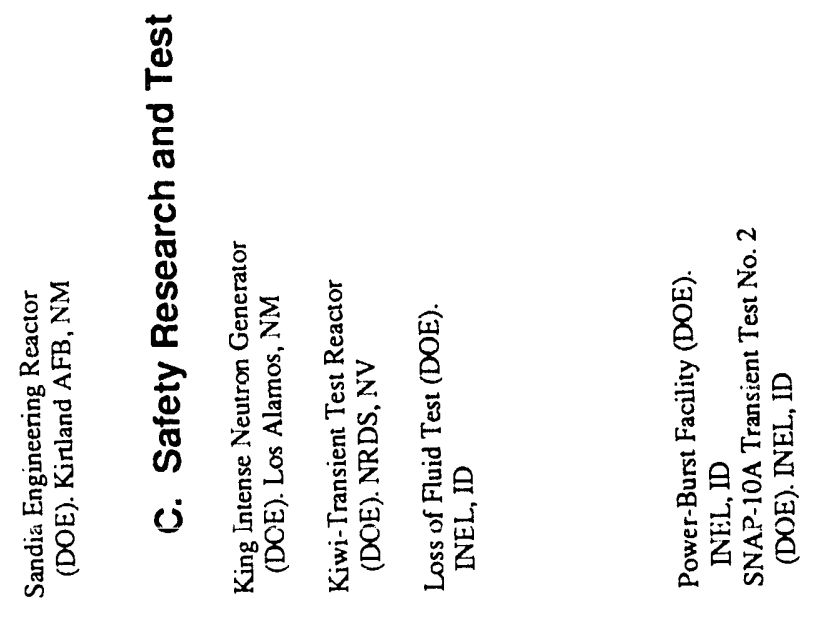

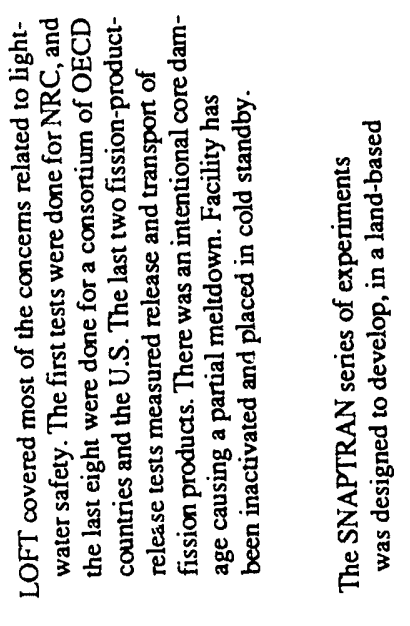
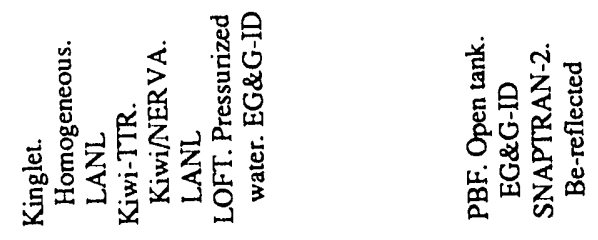

$\begin{array}{ll}8 & 0 \\ 2 & 8\end{array}$

$\begin{array}{ll}8 & 8 \\ m & n\end{array}$

0
\&̊
ì 


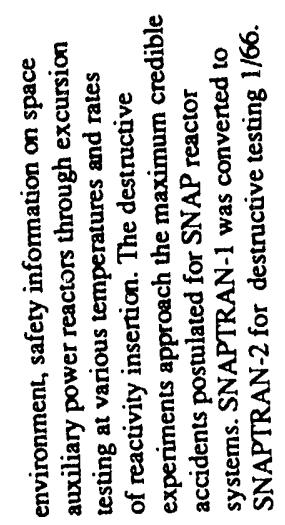

熟

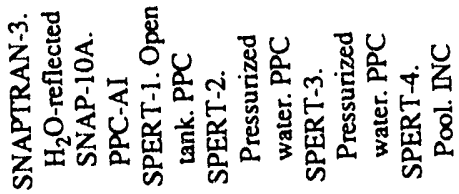

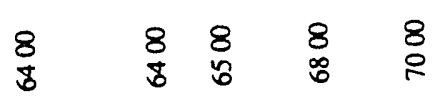

8.

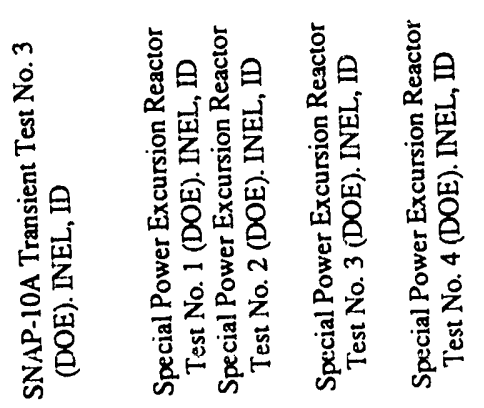

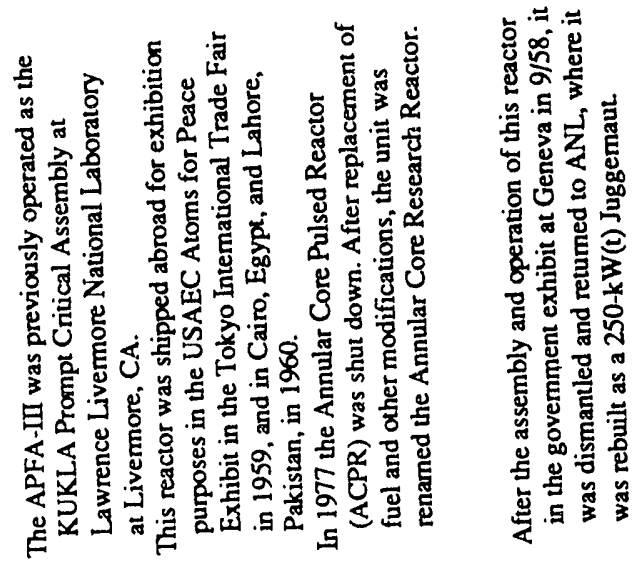

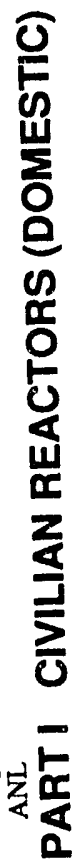

8

8 \& \& $8 \%$

$8 \quad 8$

8 宋 8

88

$\stackrel{\circ}{-}$

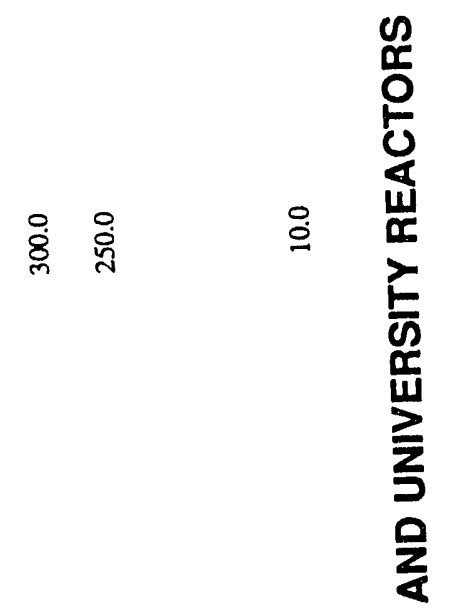

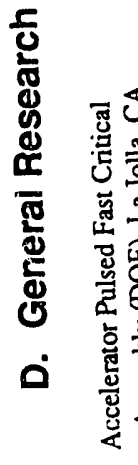

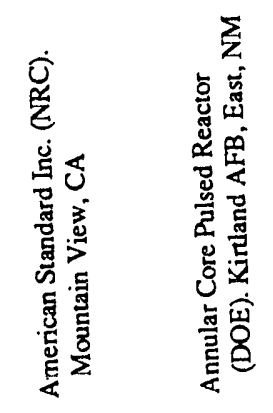

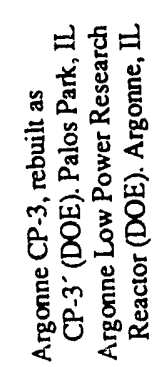

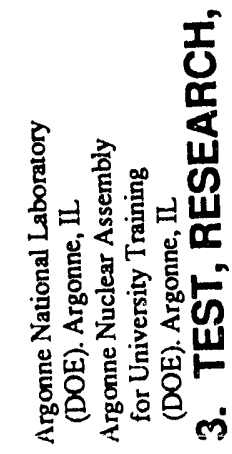




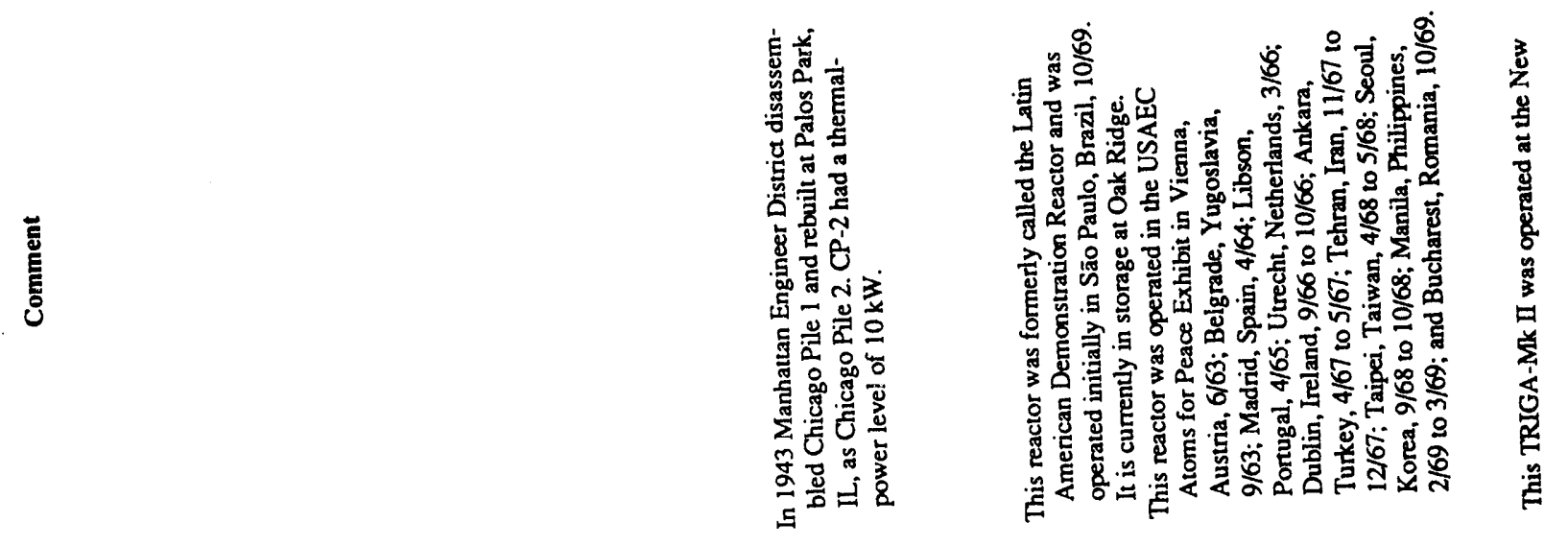

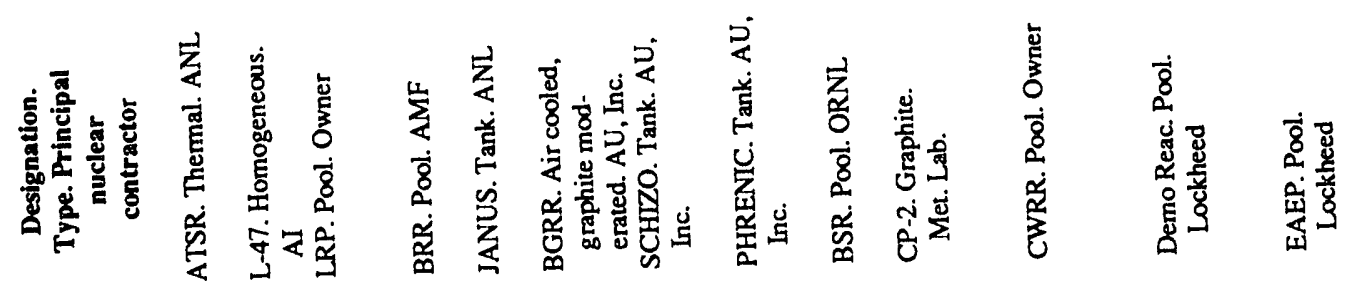

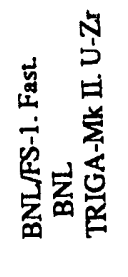

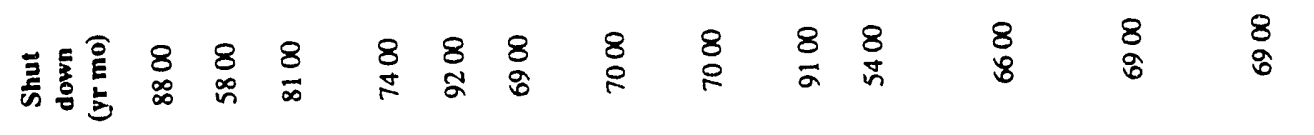

$\frac{8}{8} \frac{8}{8}$

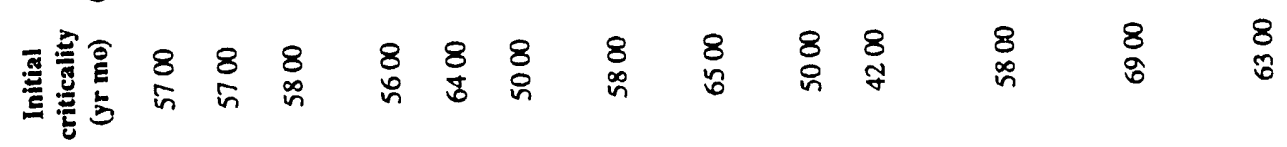

$\frac{8}{6} \frac{8}{8}$

恼部

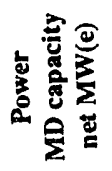

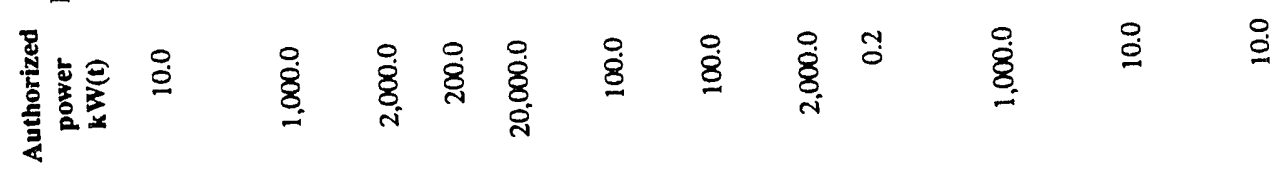

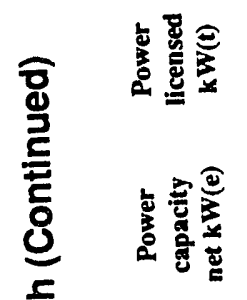

5.

离

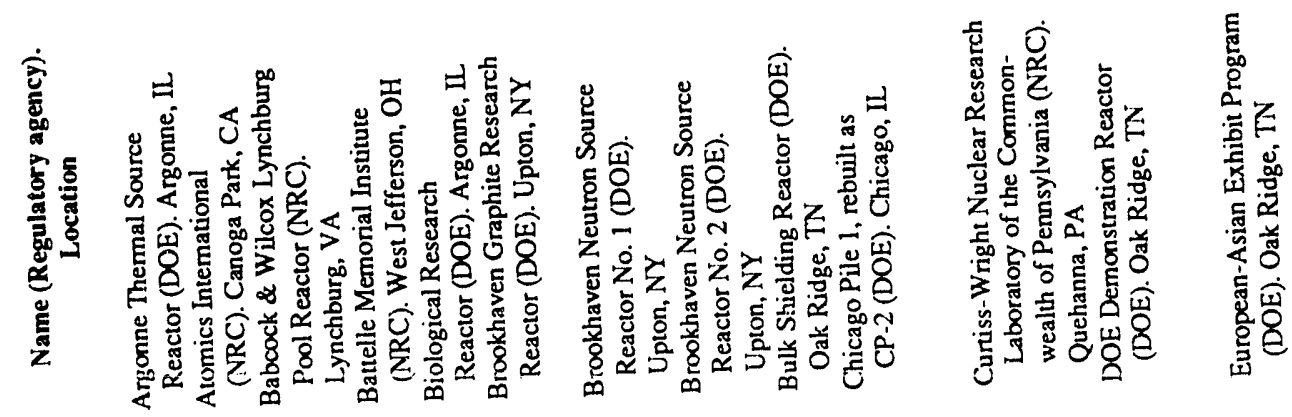

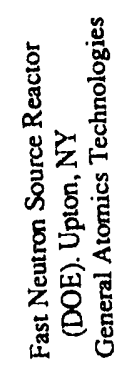



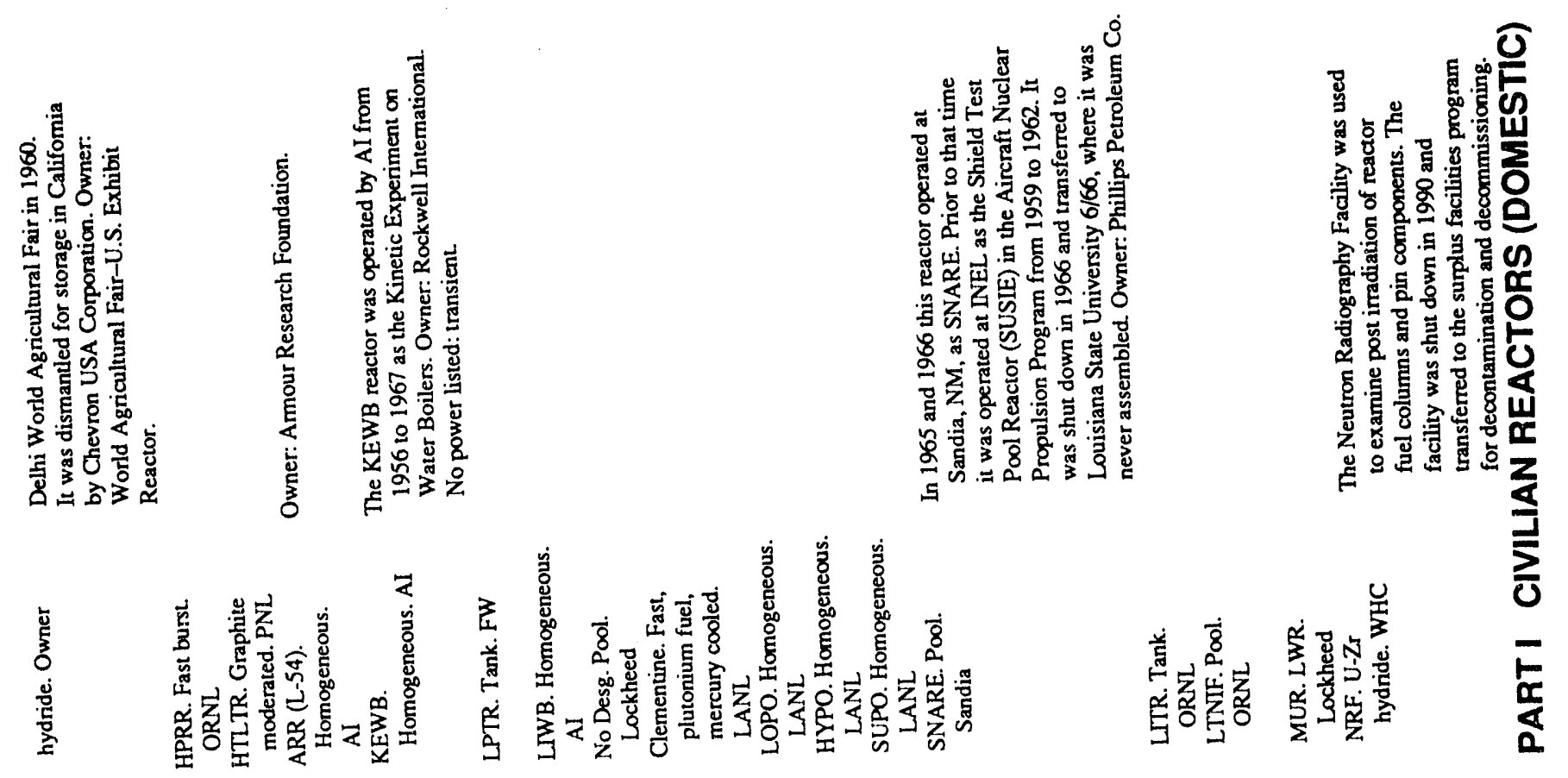

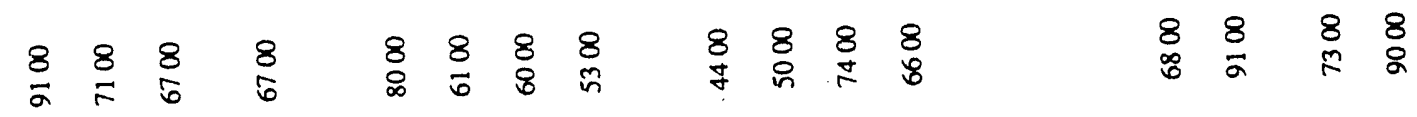

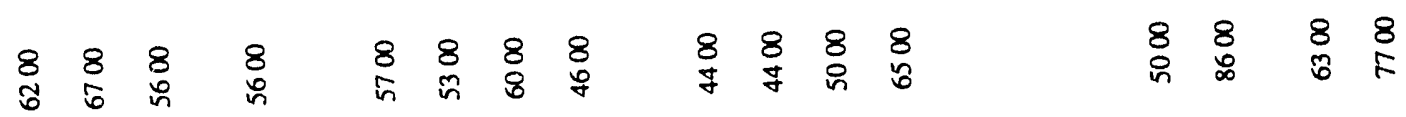

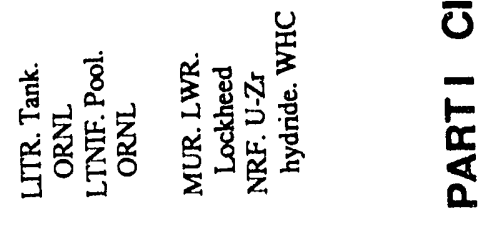

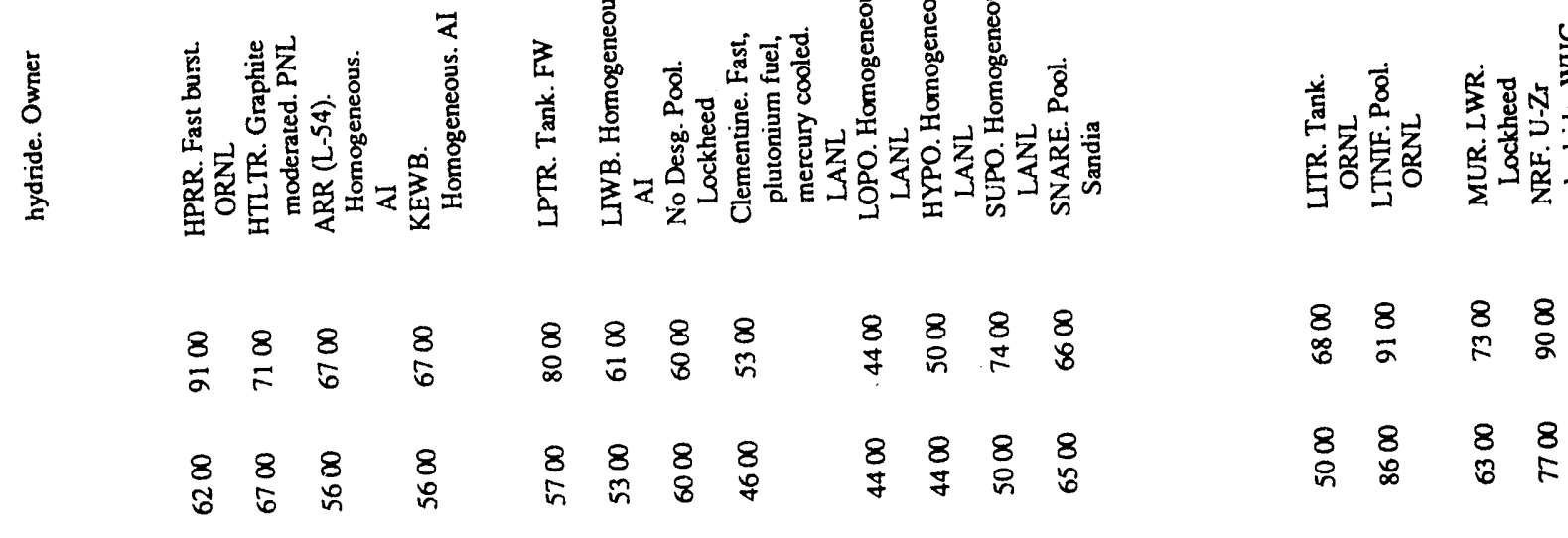

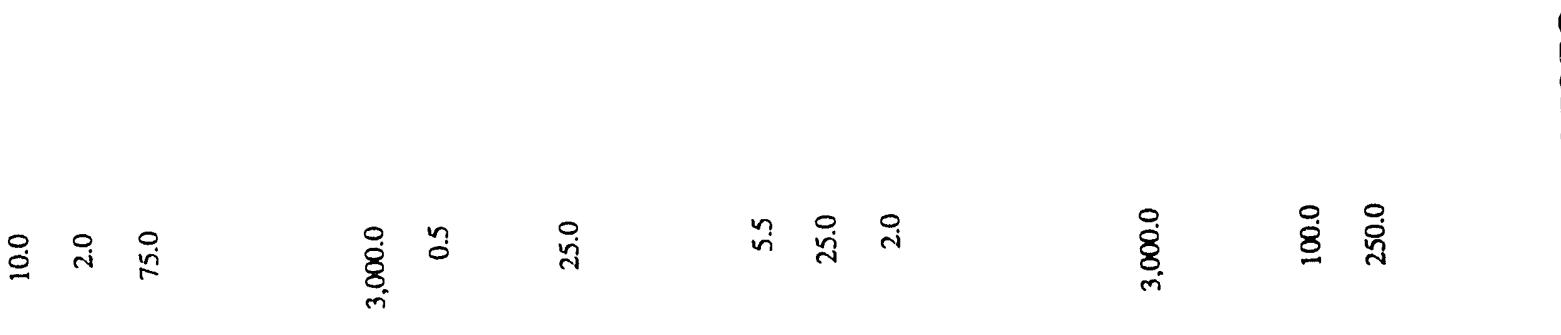



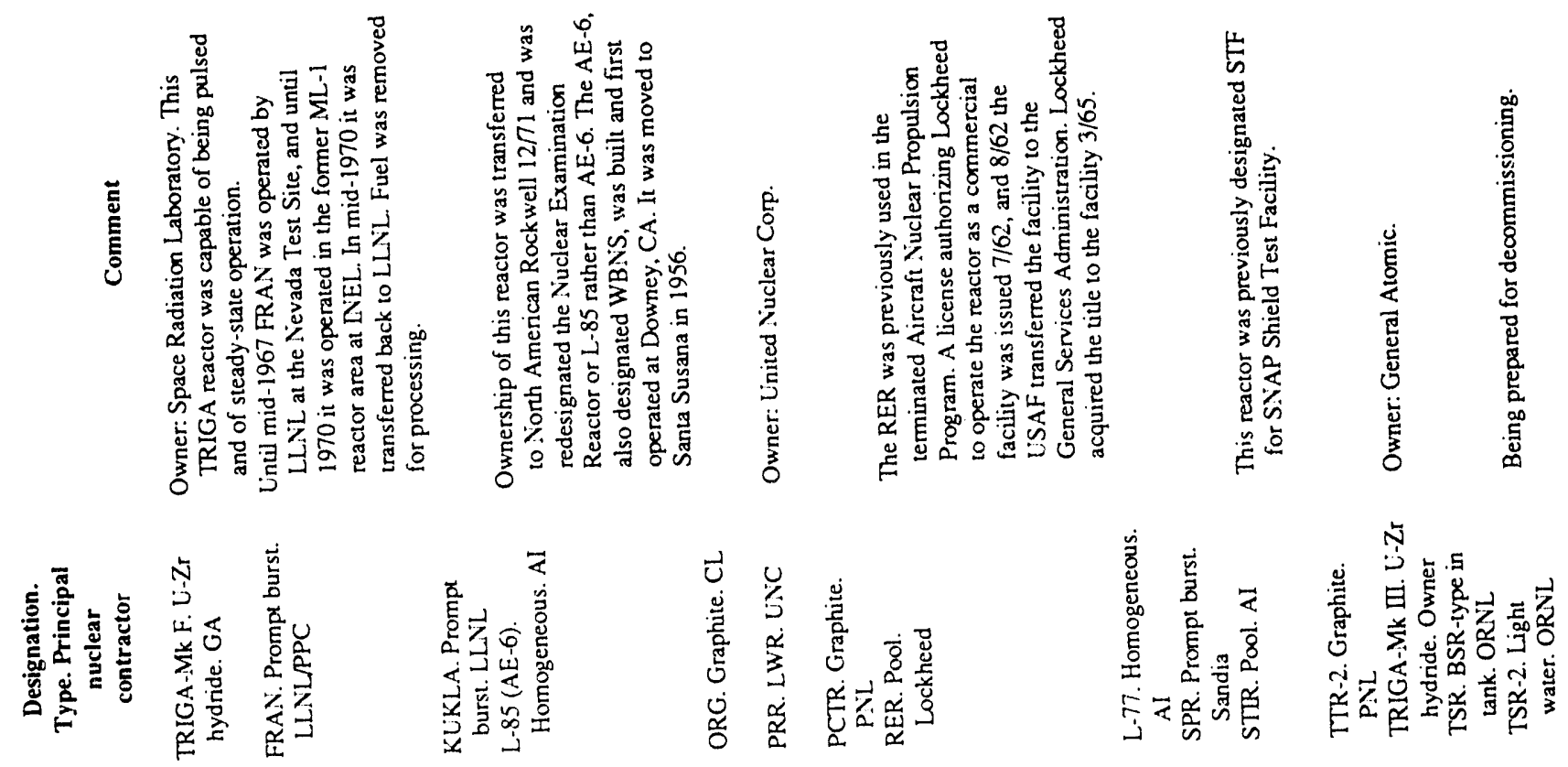

\begin{tabular}{|c|c|c|c|c|c|c|c|c|c|c|c|c|c|}
\hline 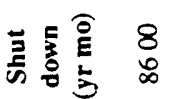 & $\begin{array}{l}8 \\
?\end{array}$ & $\begin{array}{l}8 \\
4 \\
0\end{array}$ & $\begin{array}{l}8 \\
8 \\
\infty\end{array}$ & $\begin{array}{l}8 \\
3\end{array}$ & $\frac{8}{1}$ & \begin{tabular}{l}
8 \\
\multirow{2}{*}{}
\end{tabular} & $\begin{array}{l}8 \\
8\end{array}$ & $\underset{8}{\frac{1}{2}}$ & $\begin{array}{l}8 \\
5\end{array}$ & \begin{tabular}{l}
8 \\
\multirow{2}{*}{}
\end{tabular} & $\underset{N}{8}$ & $\stackrel{8}{\%}$ & $\begin{array}{l}8 \\
\infty \\
\infty\end{array}$ \\
\hline 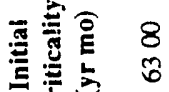 & 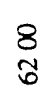 & $\begin{array}{l}8 \\
\text { on }\end{array}$ & \begin{tabular}{l}
8 \\
\multirow{N}{n}{}
\end{tabular} & $\begin{array}{l}8 \\
q\end{array}$ & $\begin{array}{l}8 \\
\infty \\
\infty\end{array}$ & $\begin{array}{l}8 \\
\text { nn }\end{array}$ & $\begin{array}{l}8 \\
\infty \\
i n\end{array}$ & $\begin{array}{l}8 \\
\infty \\
n\end{array}$ & $\frac{8}{6}$ & $\frac{8}{6}$ & $\begin{array}{l}8 \\
n \\
n\end{array}$ & $\begin{array}{l}8 \\
8\end{array}$ & $\begin{array}{l}8 \\
\text { 苟 }\end{array}$ \\
\hline
\end{tabular}

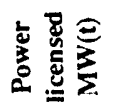

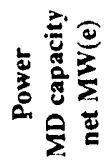

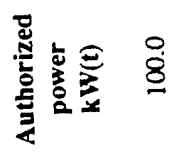

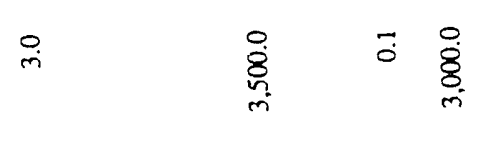

$$
\text { 횽 }
$$
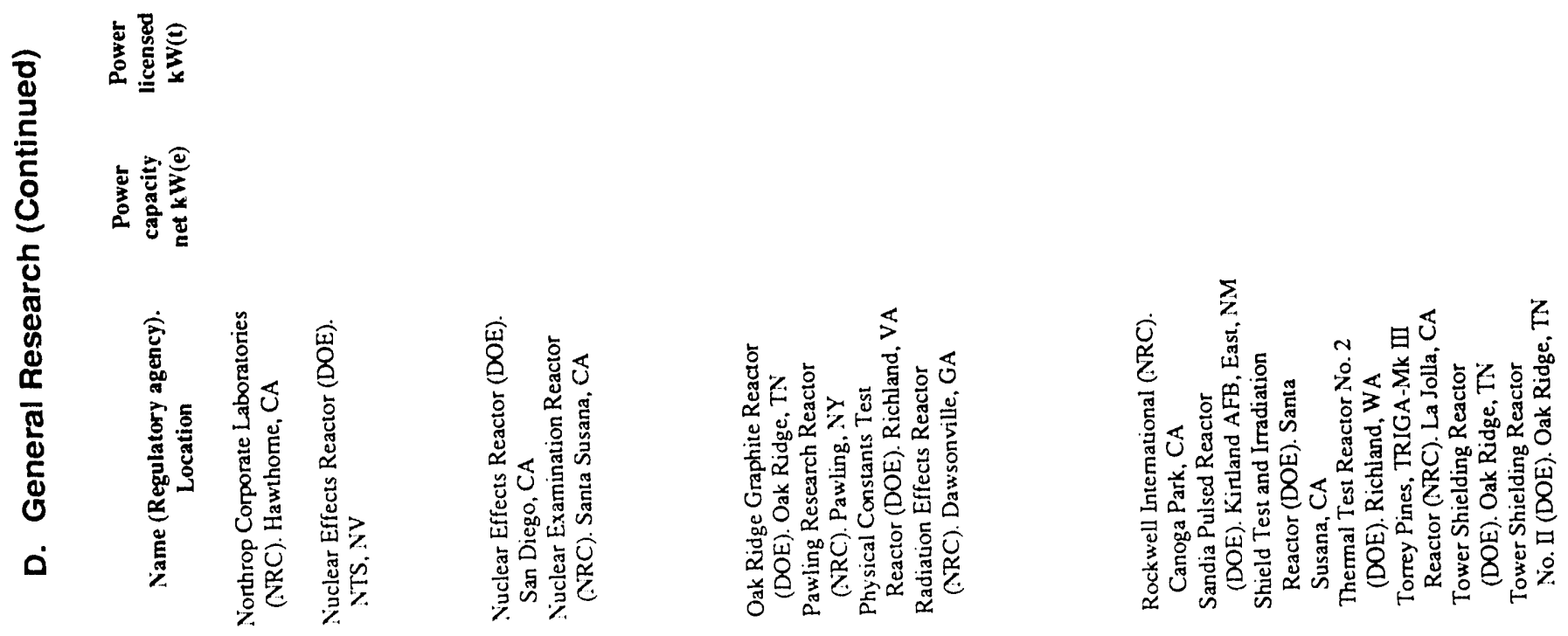


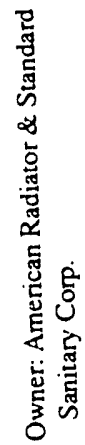

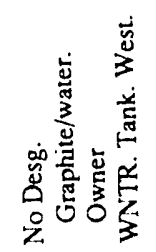

$\begin{array}{ll}8 & 8 \\ 8 & \infty\end{array}$

$\frac{8}{6} \stackrel{8}{\pi}$

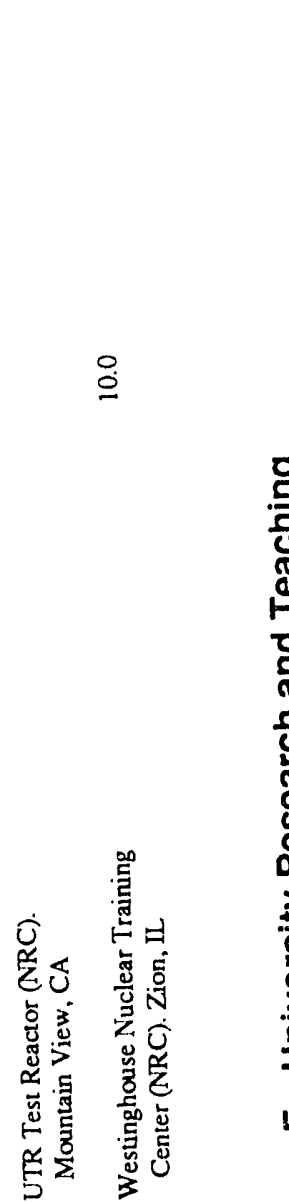

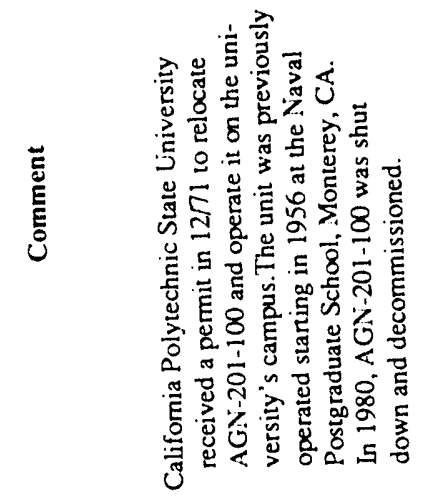

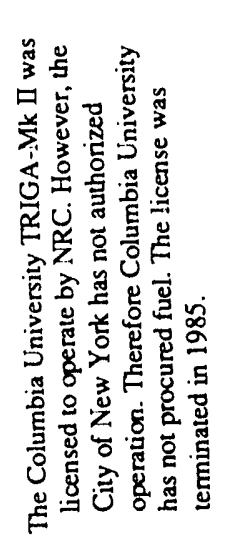

$\widetilde{\sigma}$

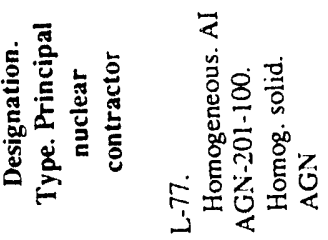

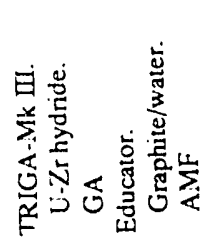

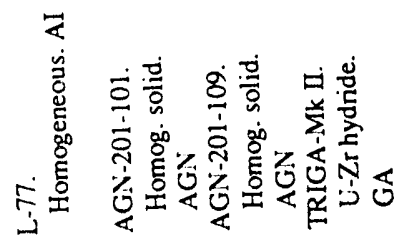

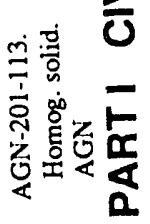

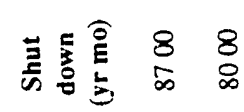

\begin{tabular}{llllll}
2 & 8 & 8 & 8 & 8 & 8 \\
\hdashline & 5 & $\infty$ & 0 & 1 & $\infty$
\end{tabular}

$\underset{\substack{8 \\ \infty}}{\infty}$

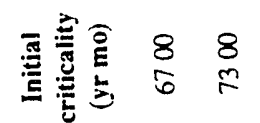

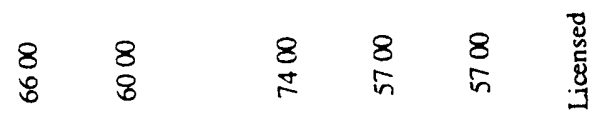

$\underset{\infty}{8}$

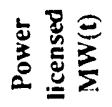

焉窟

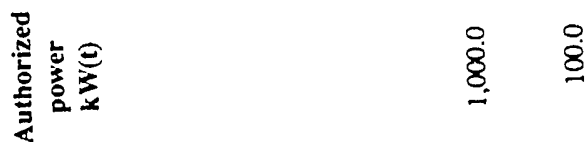

㝘

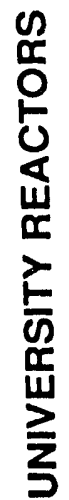

兄

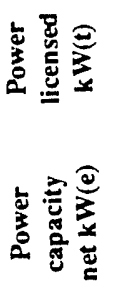

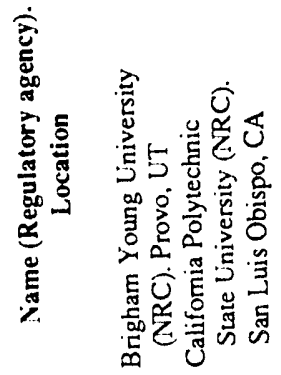

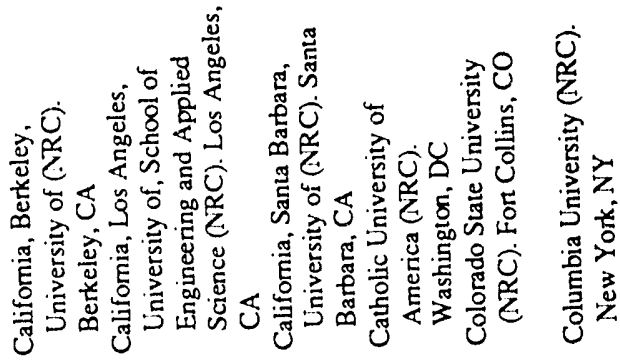



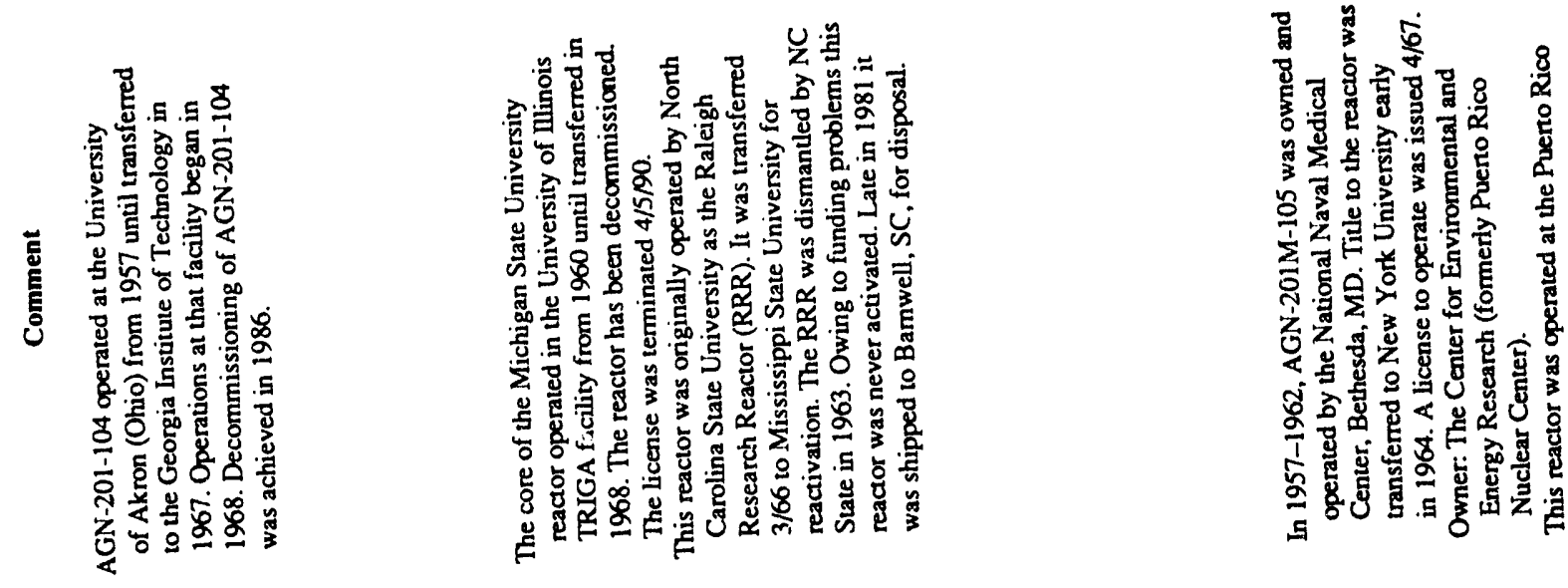

M!

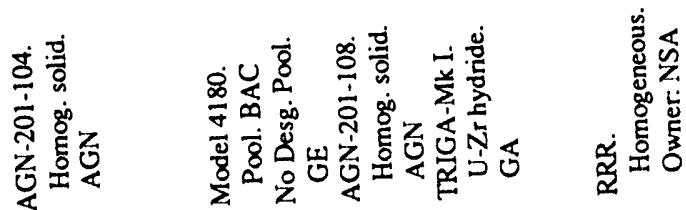

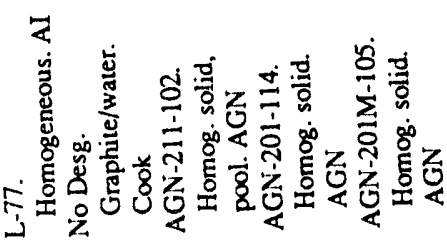

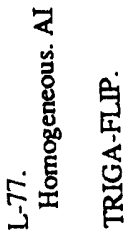

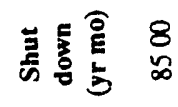

$\begin{array}{llll}8 & 8 & 1 & 8 \\ 8 & 8 & 8 & 8 \\ 8 & 8 & 8\end{array}$

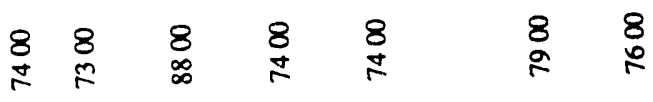

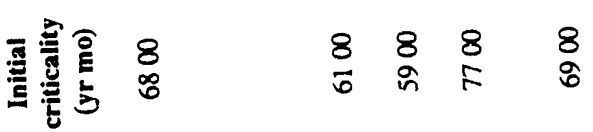

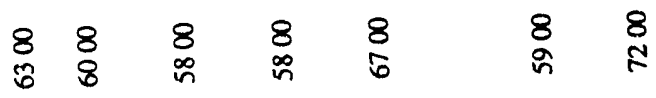

产蜜焉

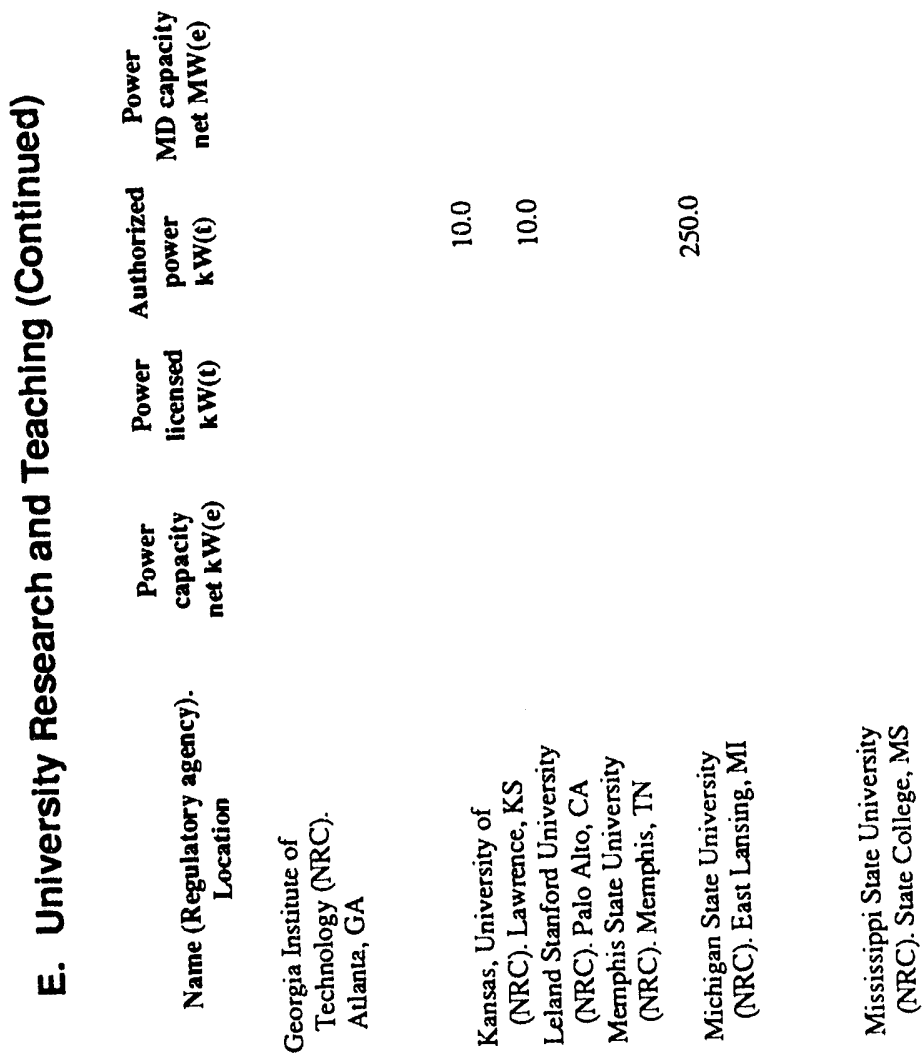

$\stackrel{8}{3}$

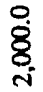

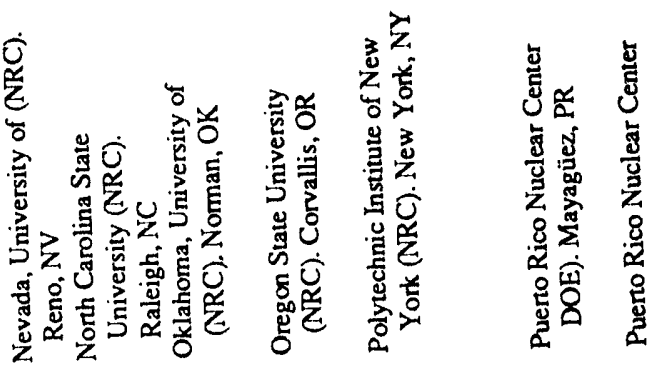




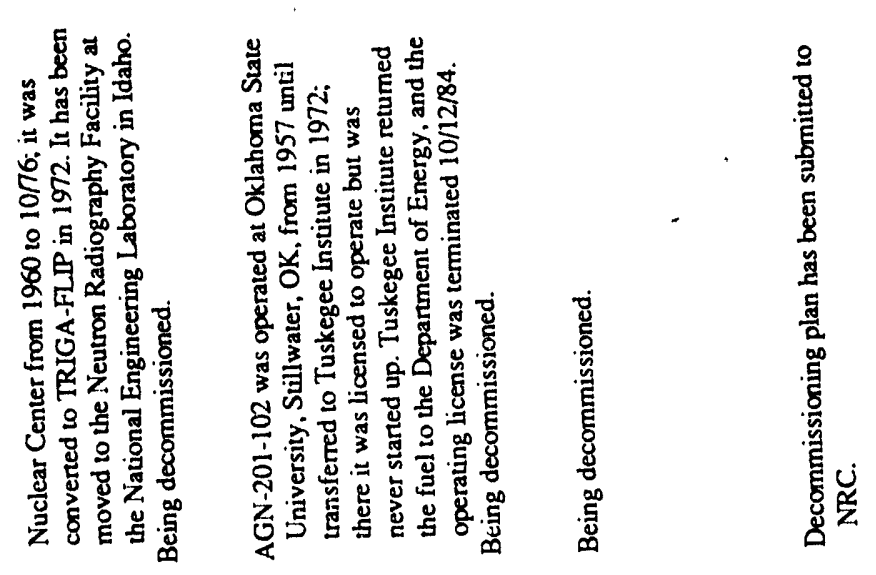

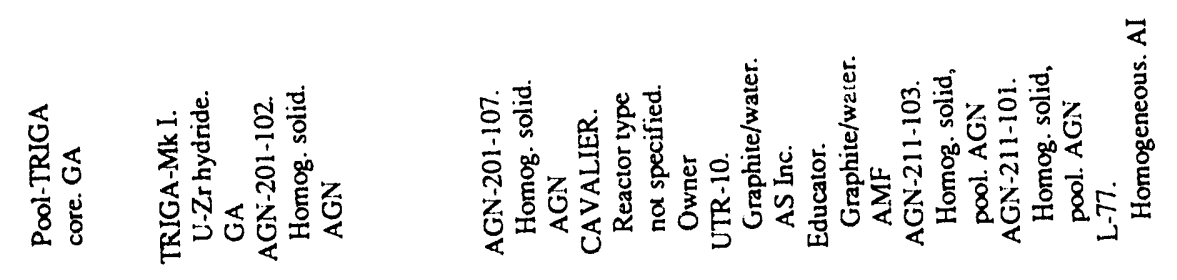

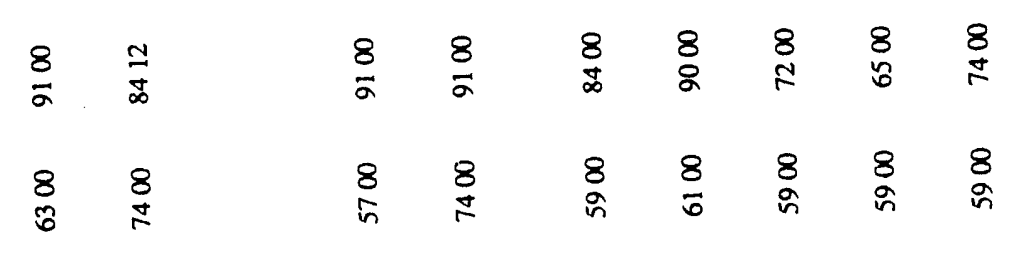

ஓ্口

\&.

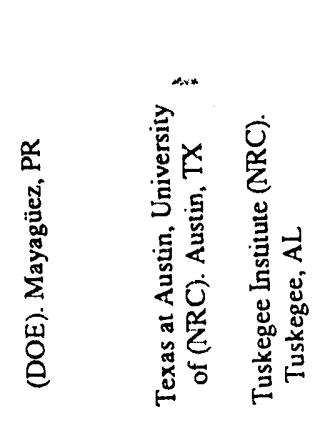

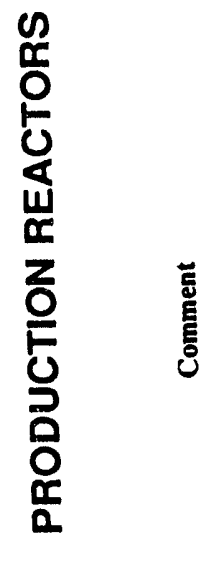

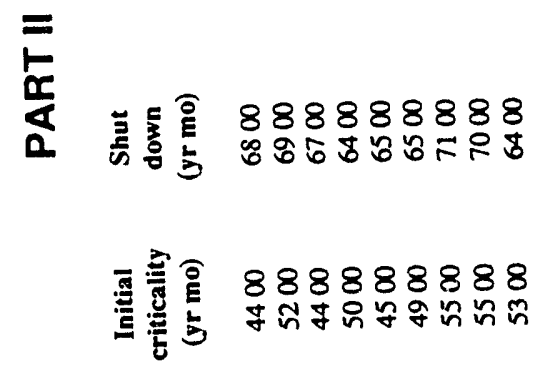

繁旁言

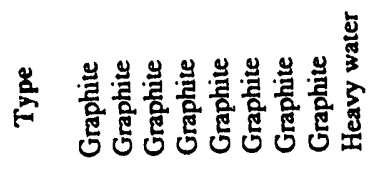

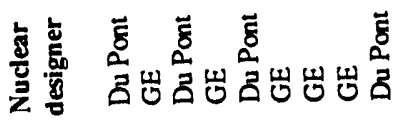
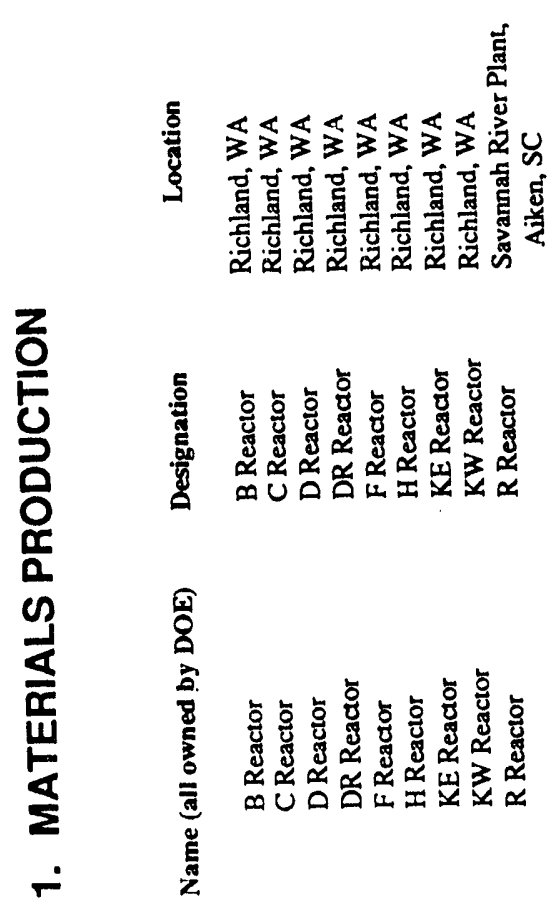


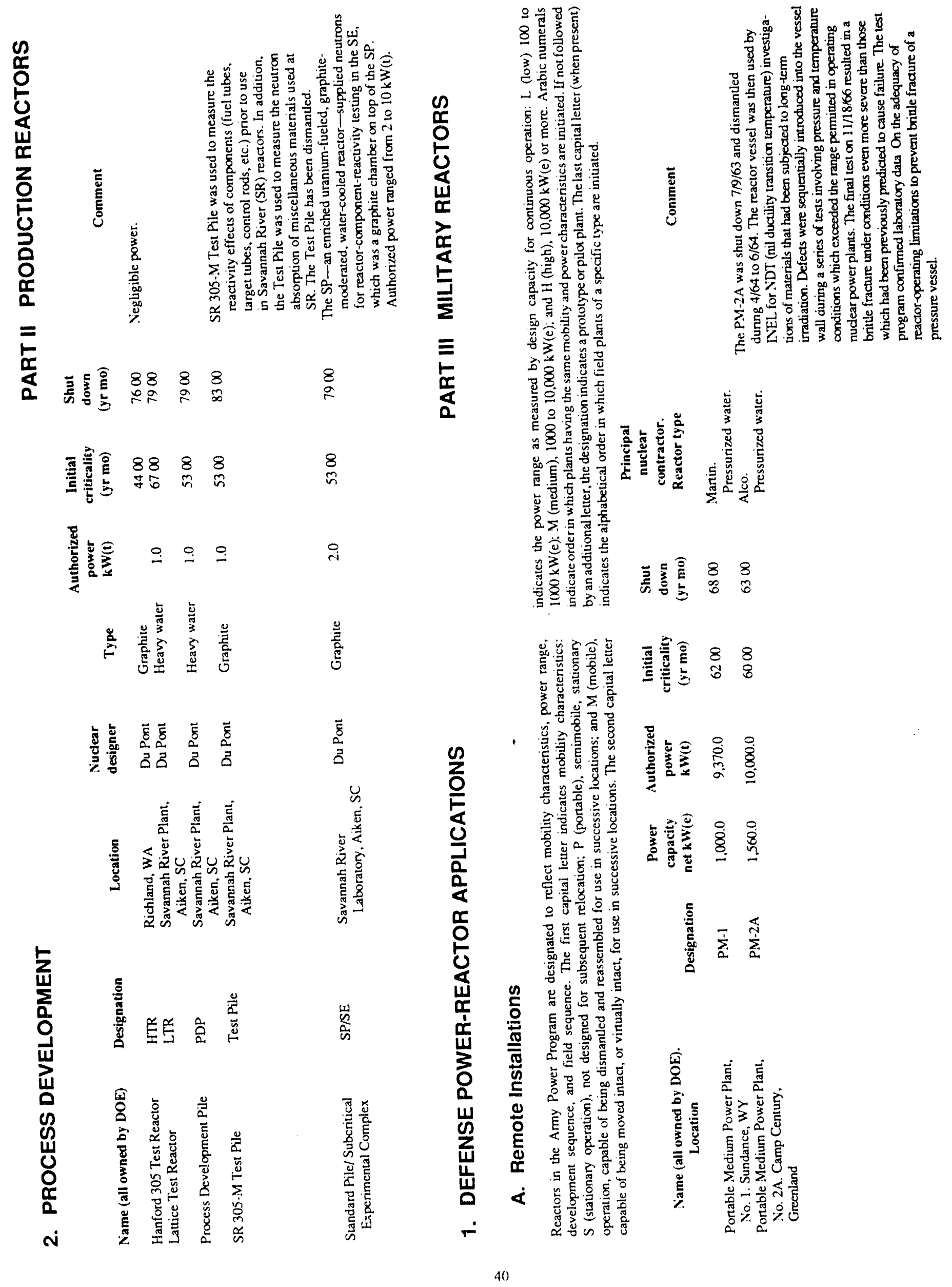




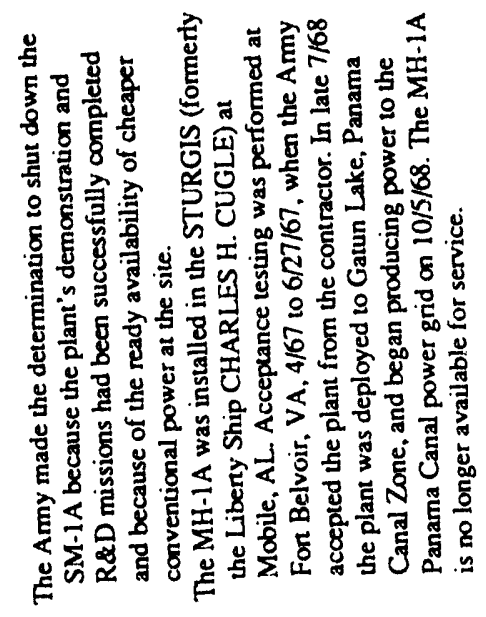

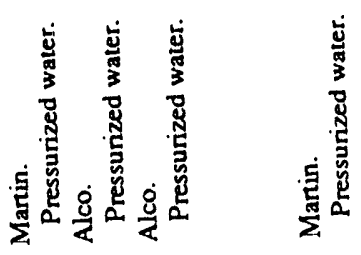

$\begin{array}{llll}8 & 8 & 8 & 1\end{array}$

$\begin{array}{llll}8 & 8 & 8 & 8 \\ 1 & 5 & 6 & 5\end{array}$

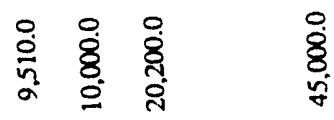

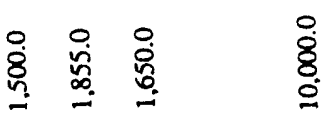

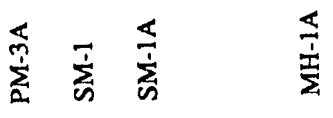

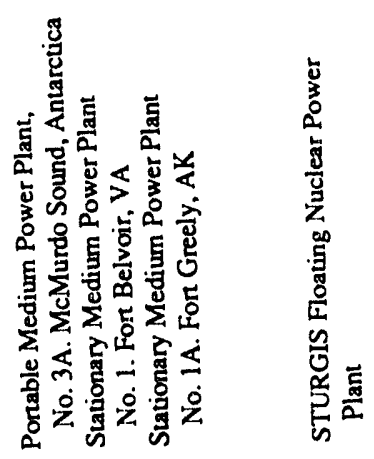

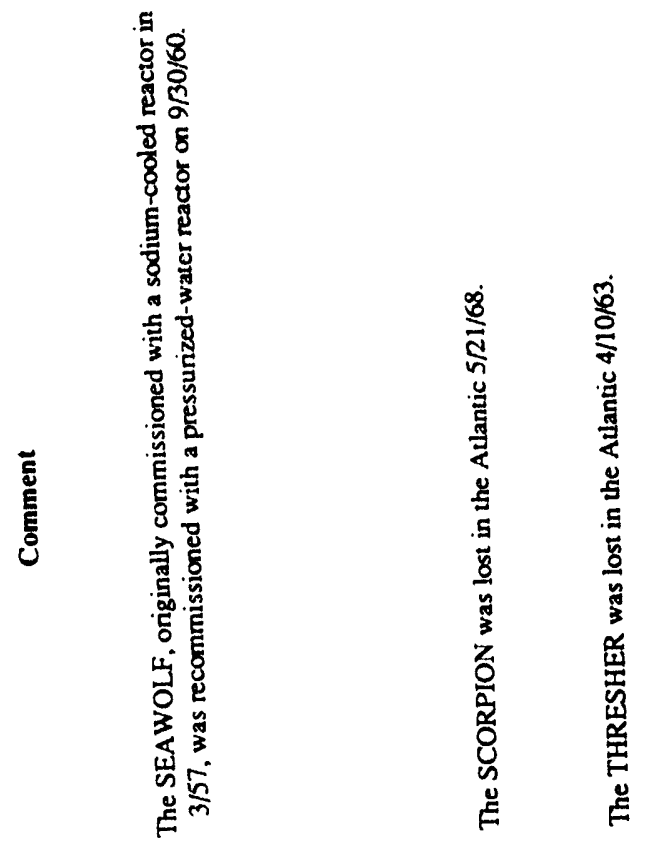

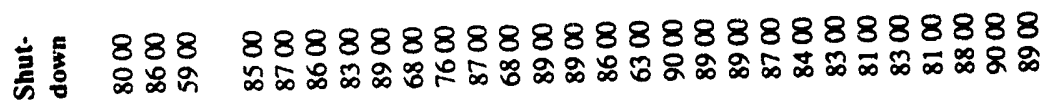

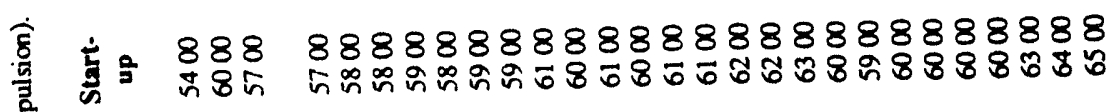

范

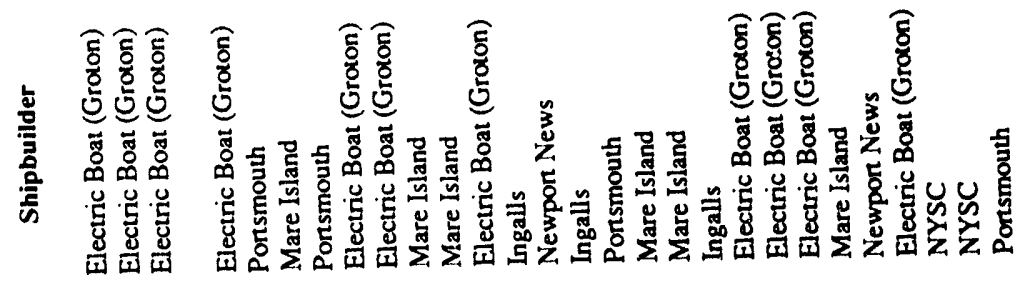

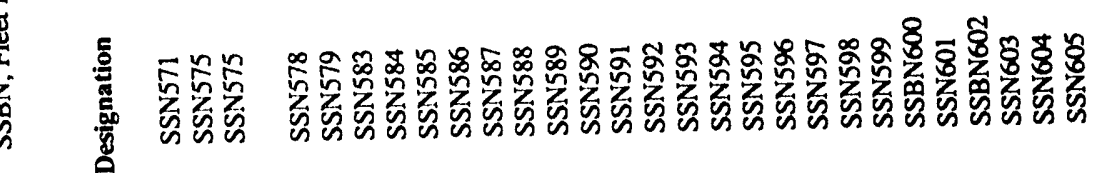

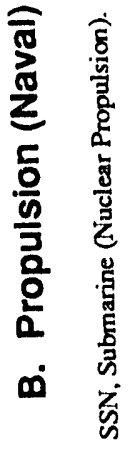

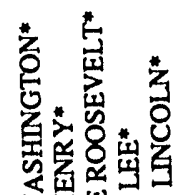

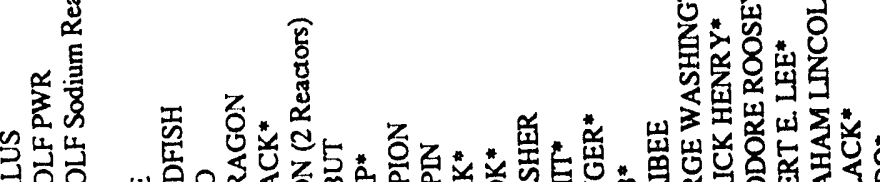

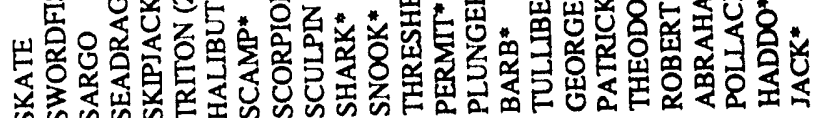




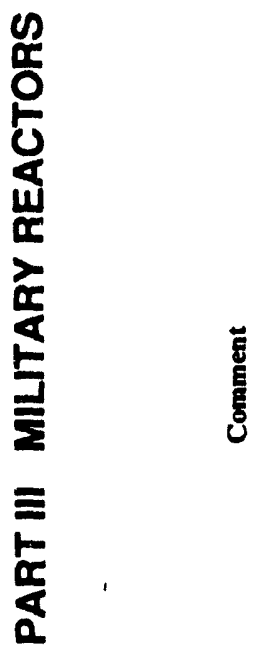

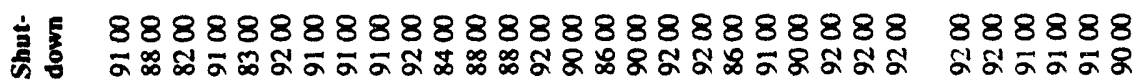

을 8888888888888888888888888 888888

年

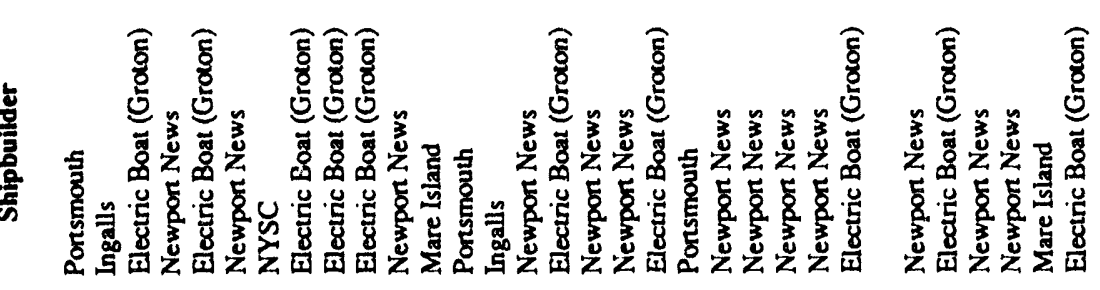



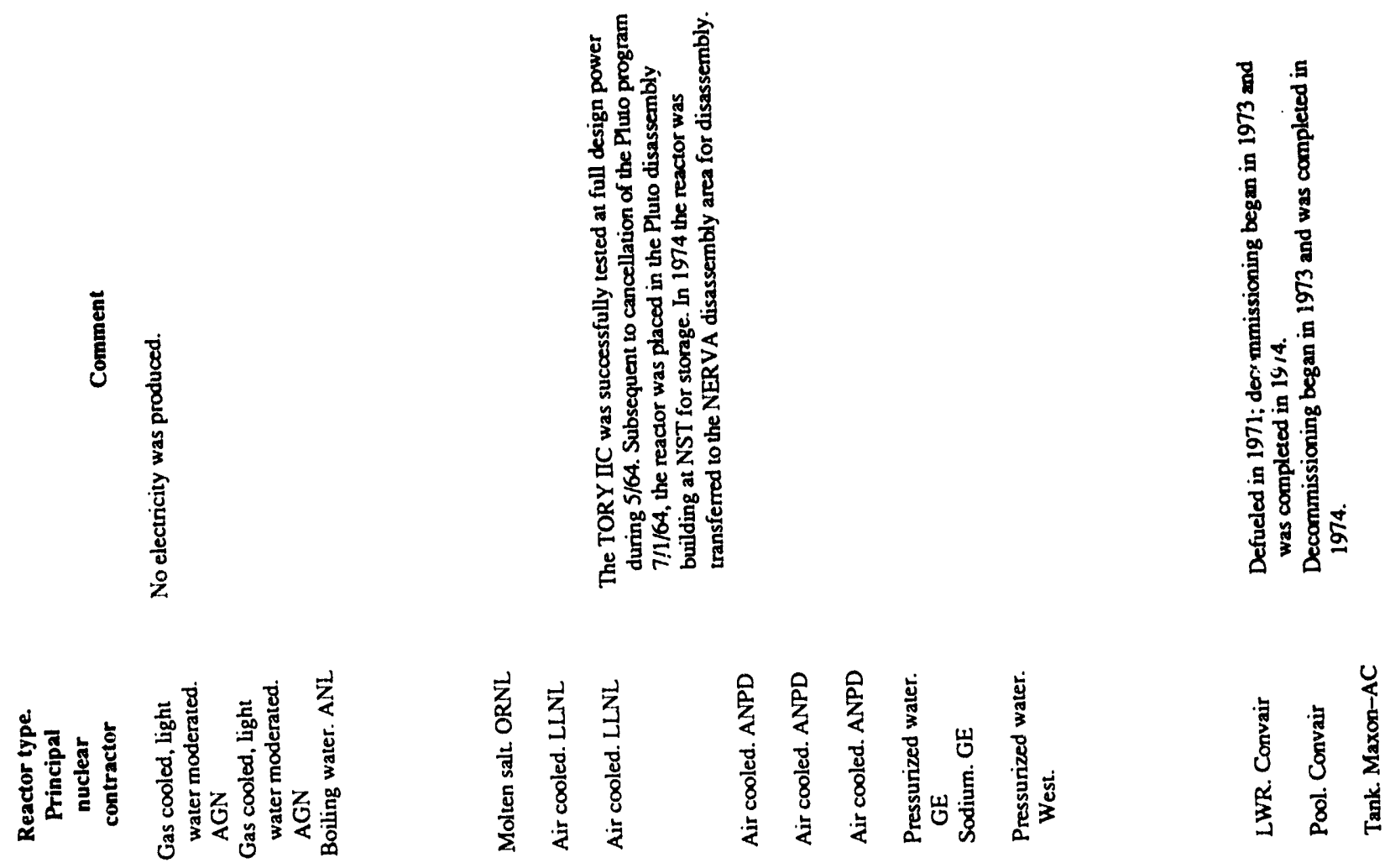

폭ำ

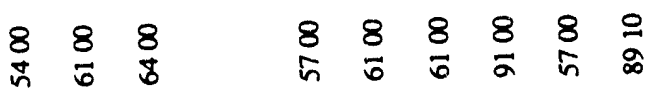

8
$7 \%$

ำ

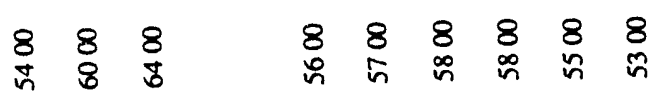

$\begin{array}{lll}8 & 8 & 8 \\ \text { ท } & \text { n } & 6\end{array}$

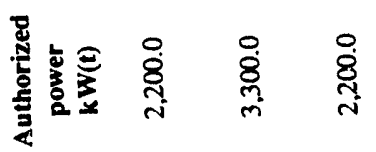

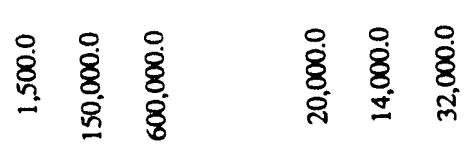

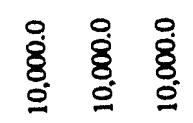

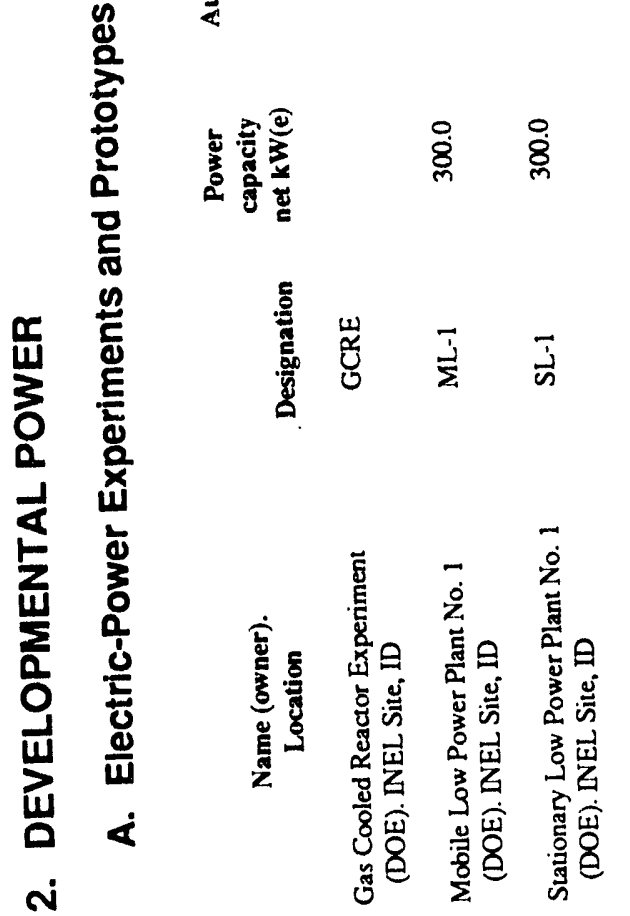

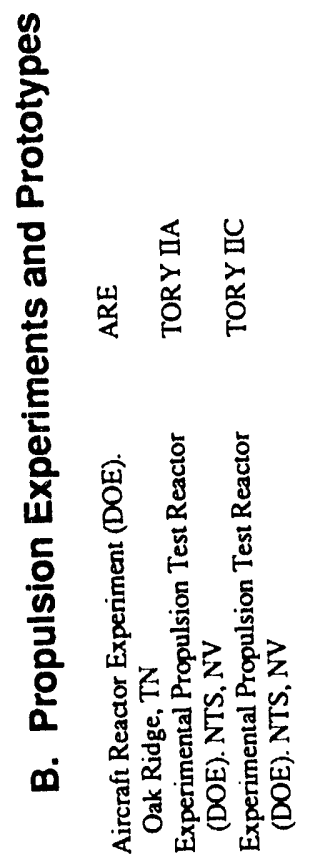

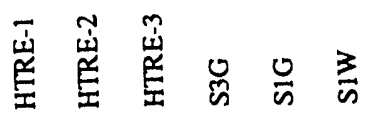

菖总离

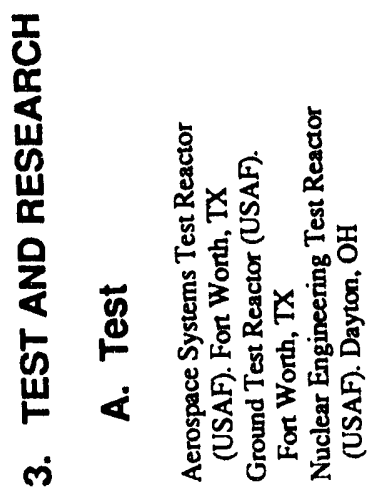



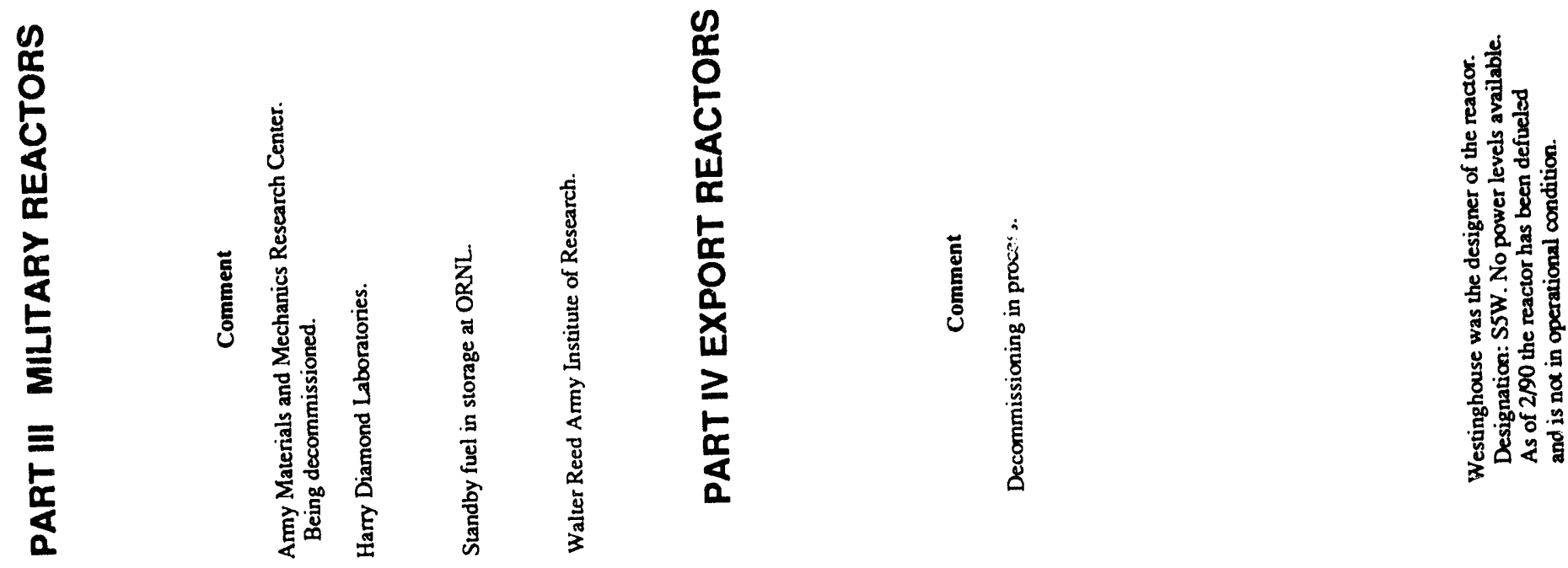

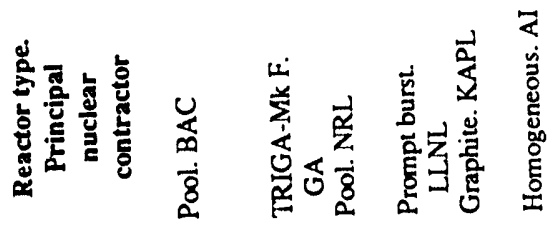

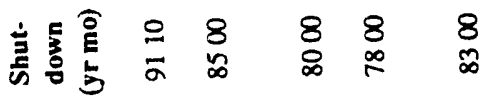

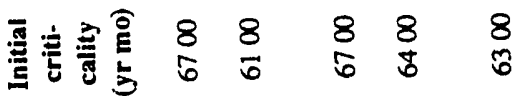

잉

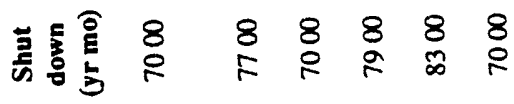

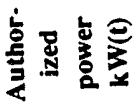

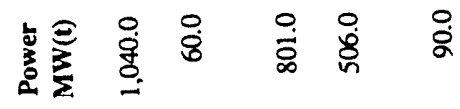

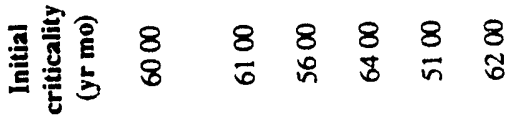

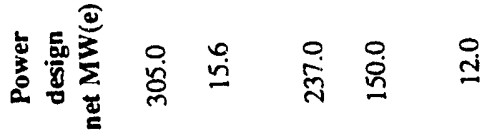

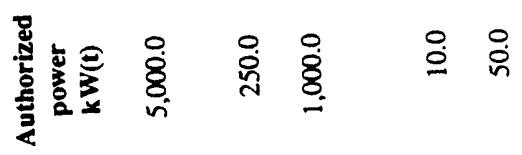

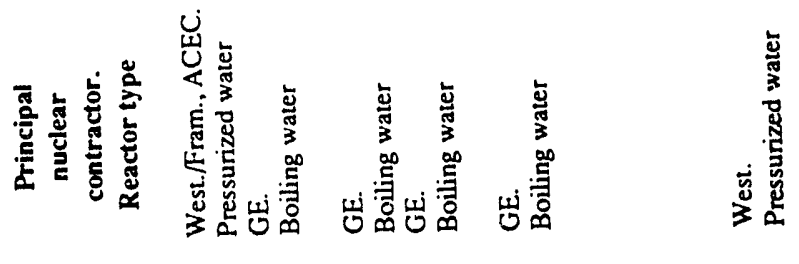

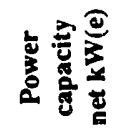

$\stackrel{2}{\frac{\pi}{2}}$

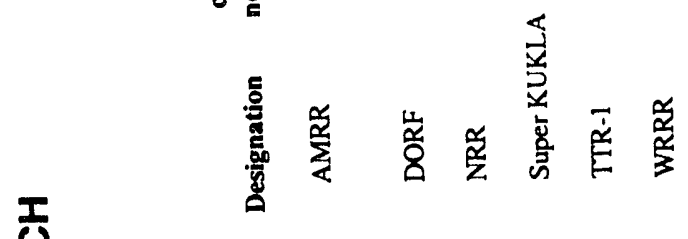

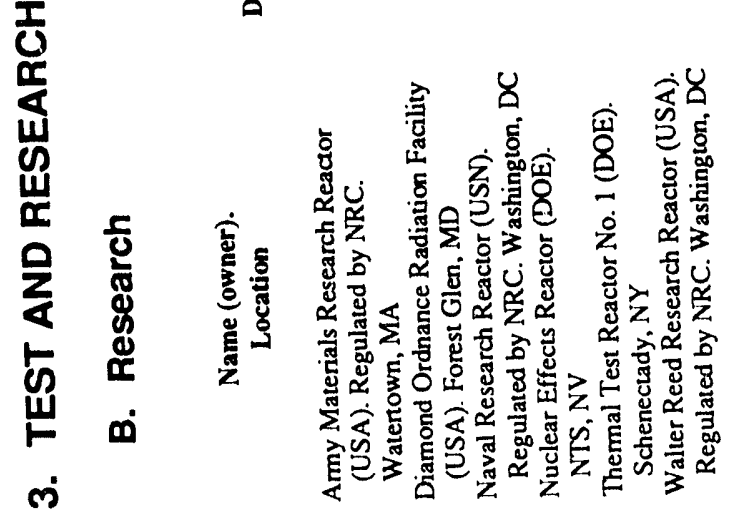

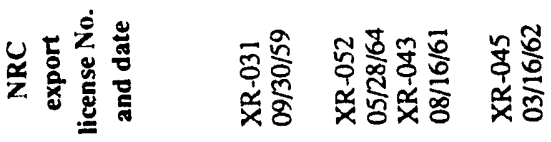

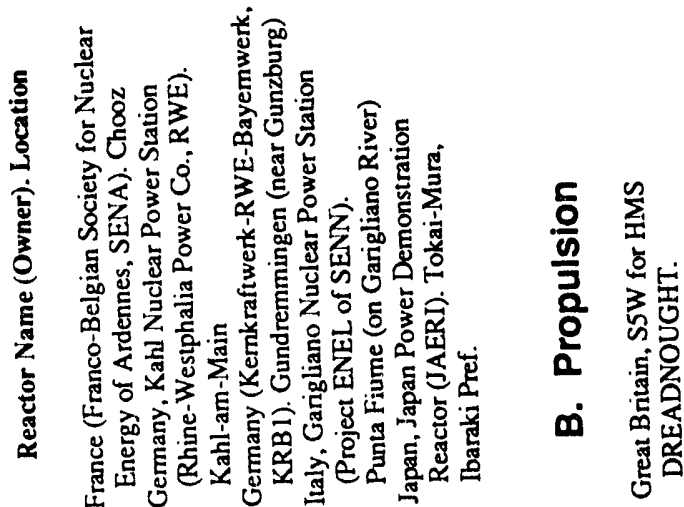




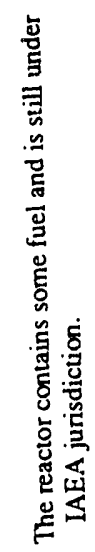

8.

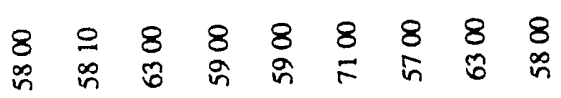

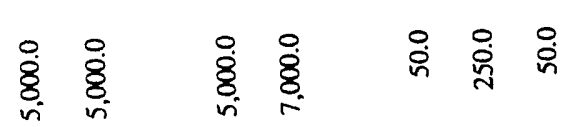

$\stackrel{\overbrace{}}{\varrho}$

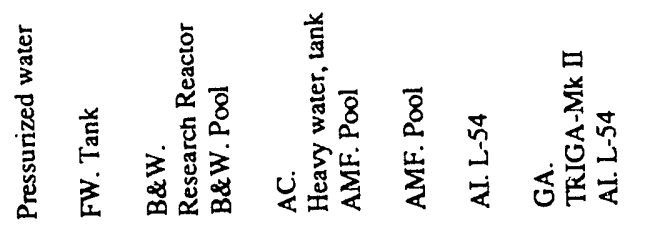

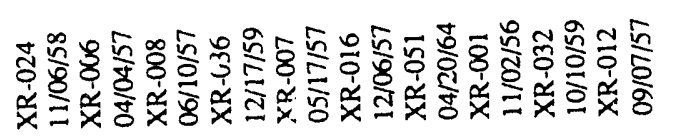

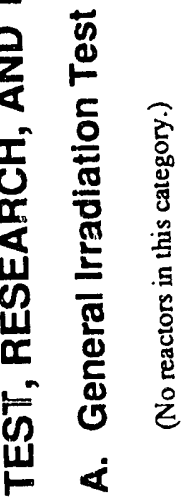

i

品

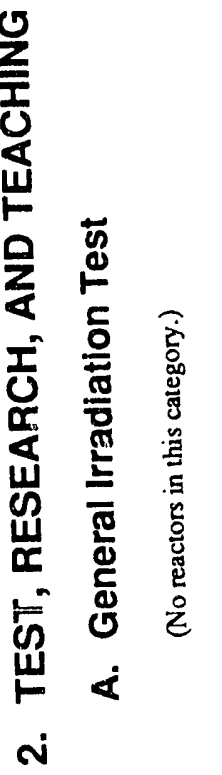

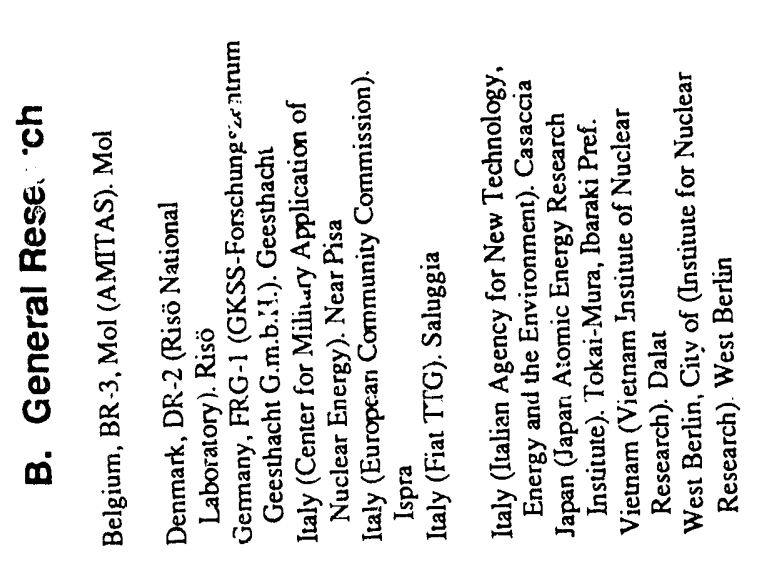

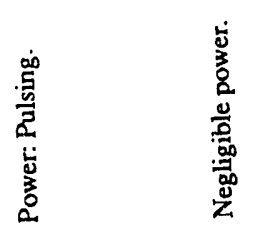

$\begin{array}{ll}8 & 8 \\ \infty & F\end{array}$

$\begin{array}{ll}1 & 8 \\ 8 & 8\end{array}$

8.

용 윰

$\stackrel{0}{8}$
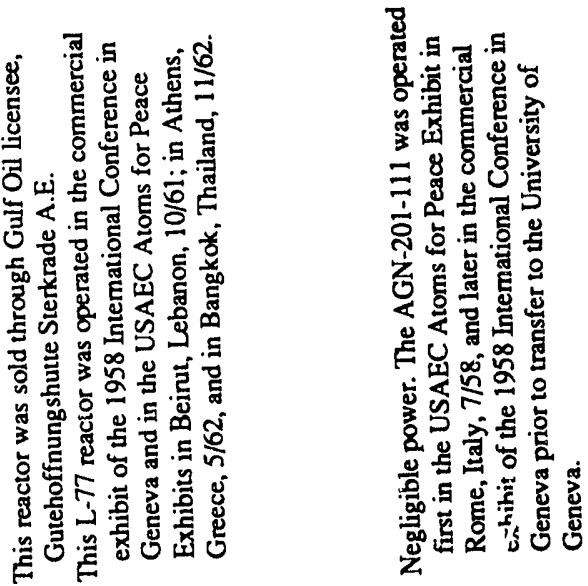

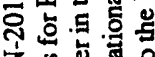

远焉

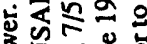

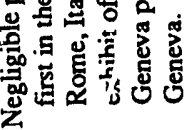

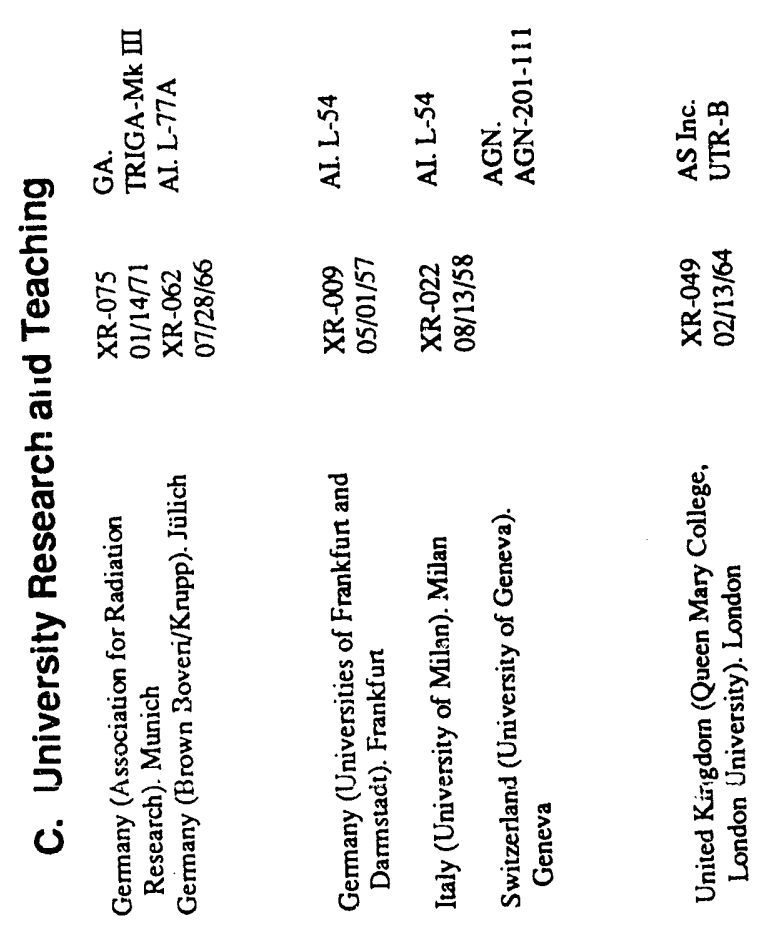



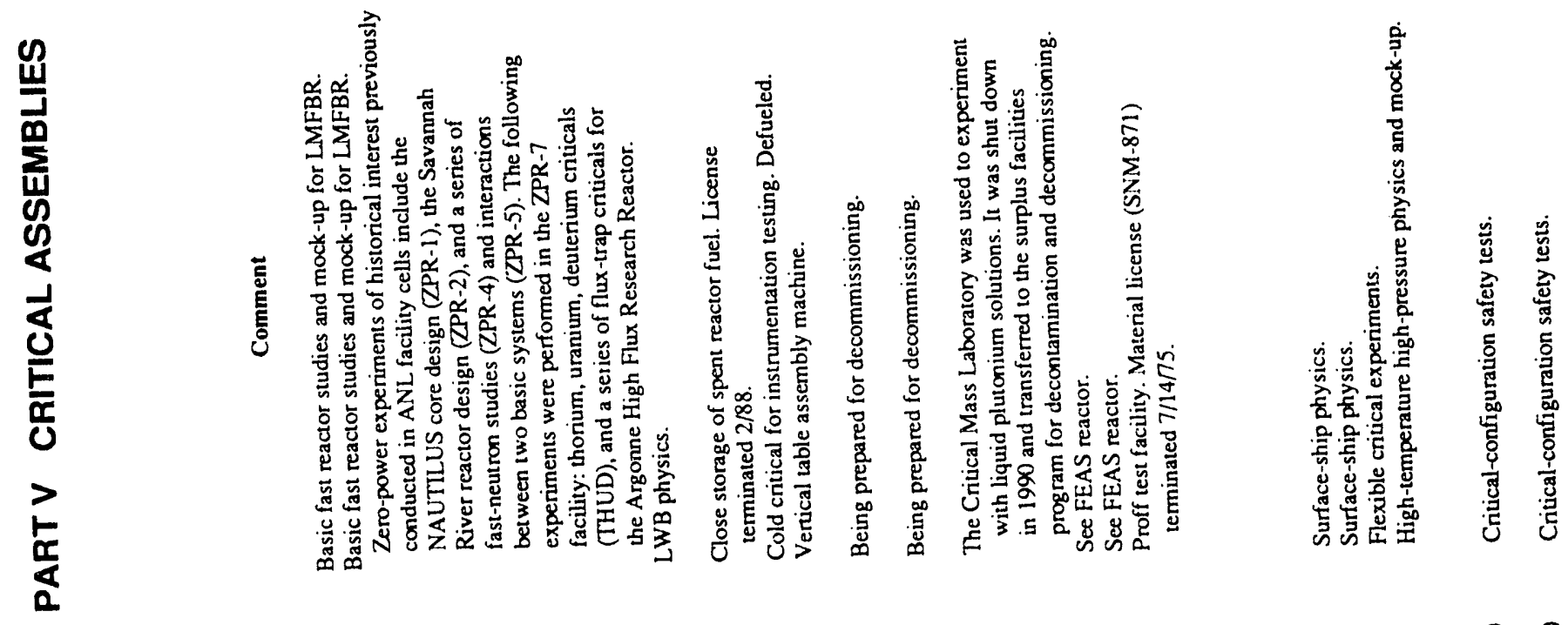

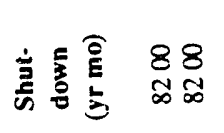

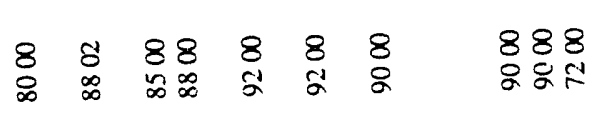

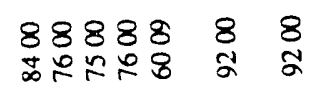

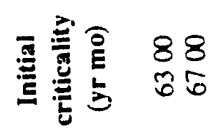

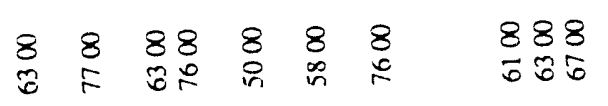

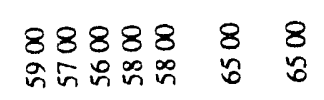

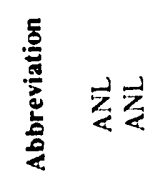

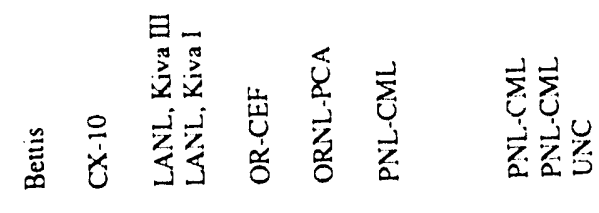

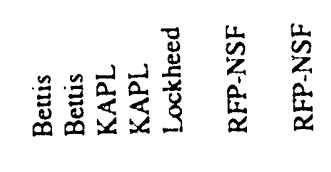
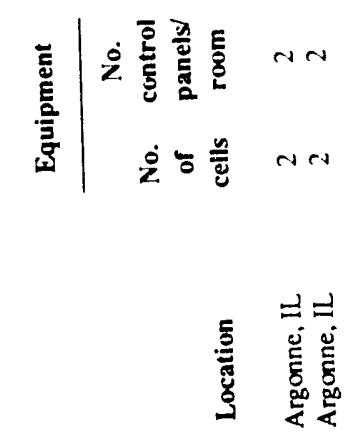

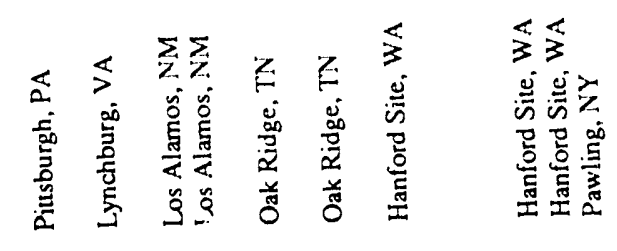

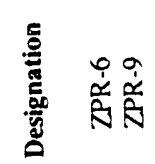

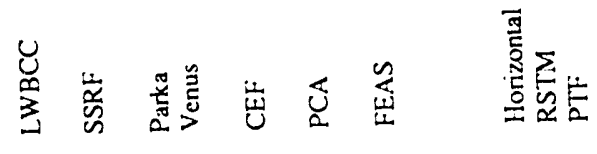
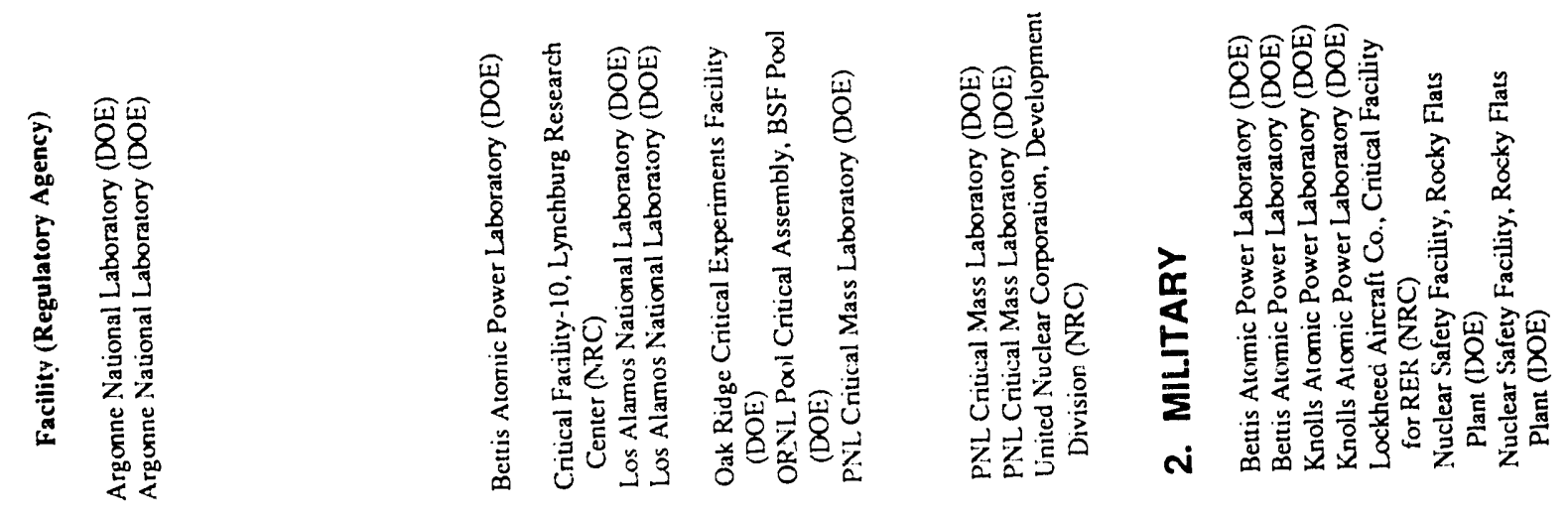
REACTOR INDEX 


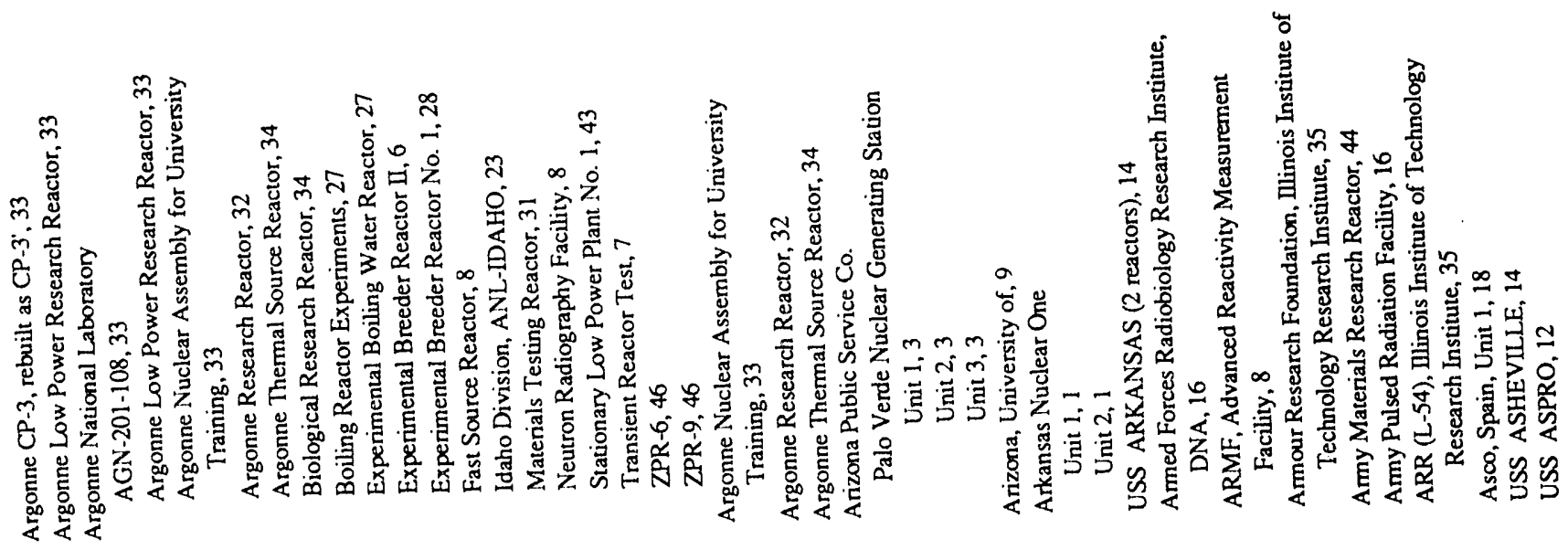

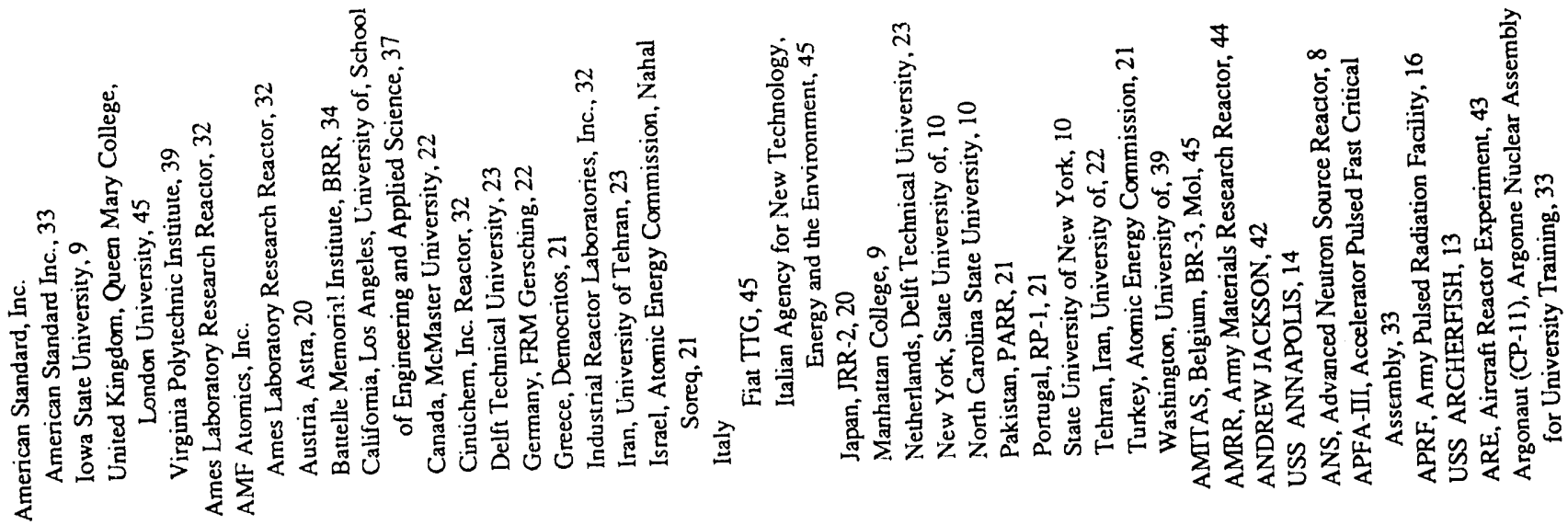

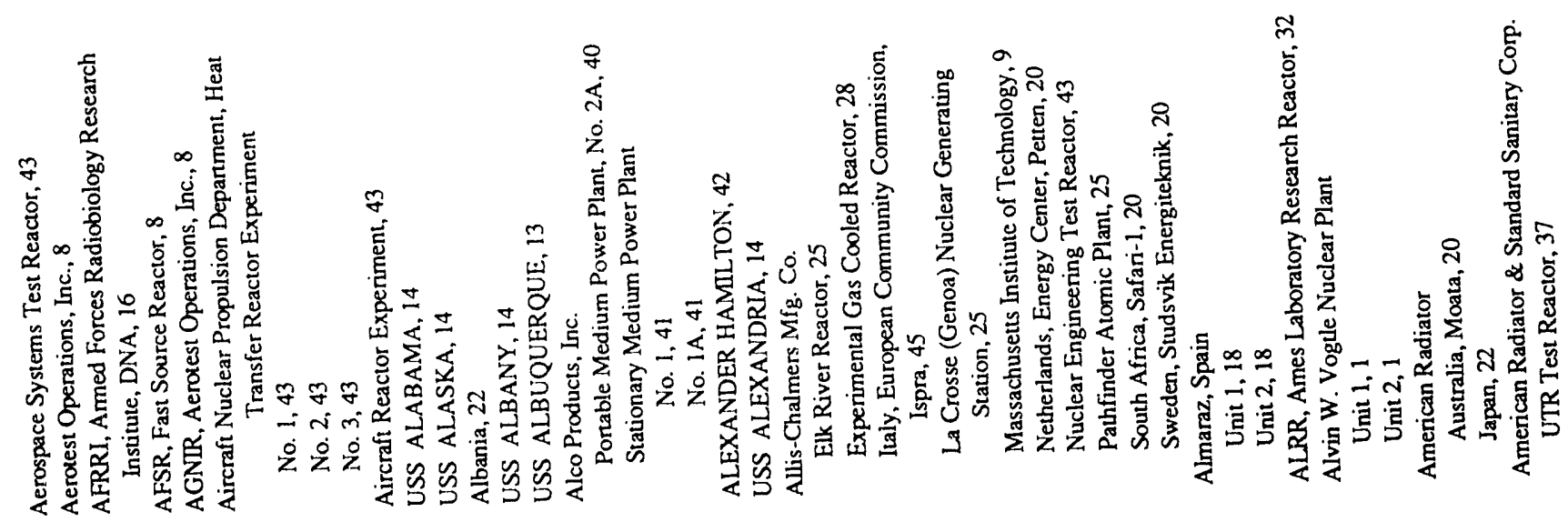

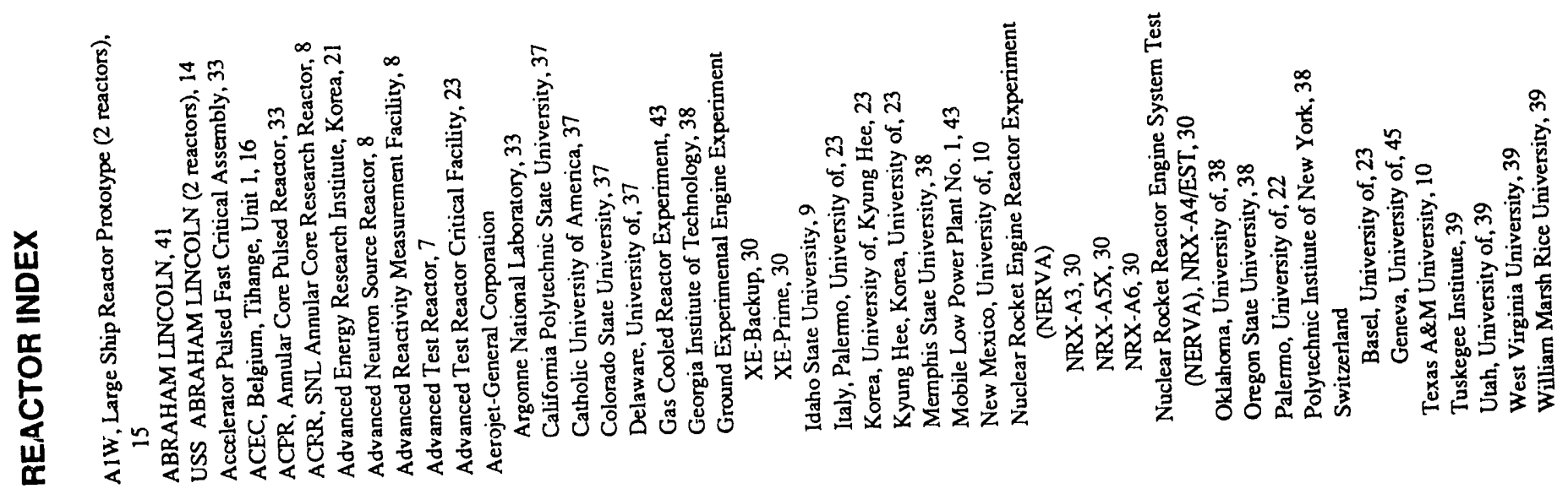




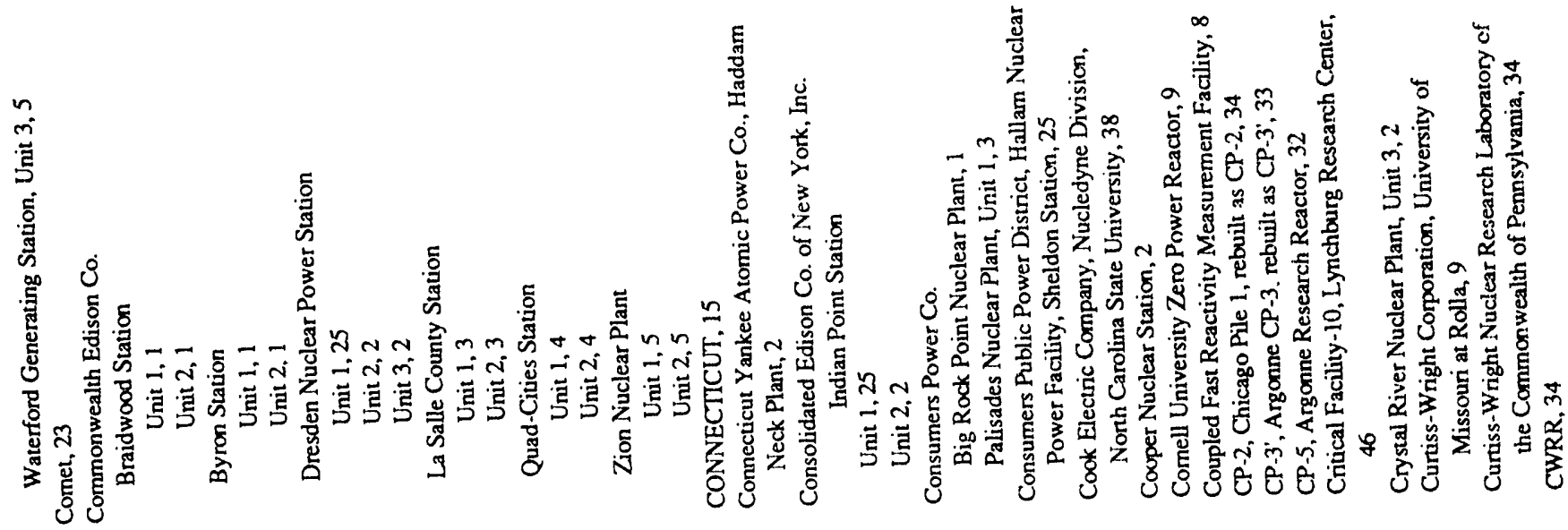

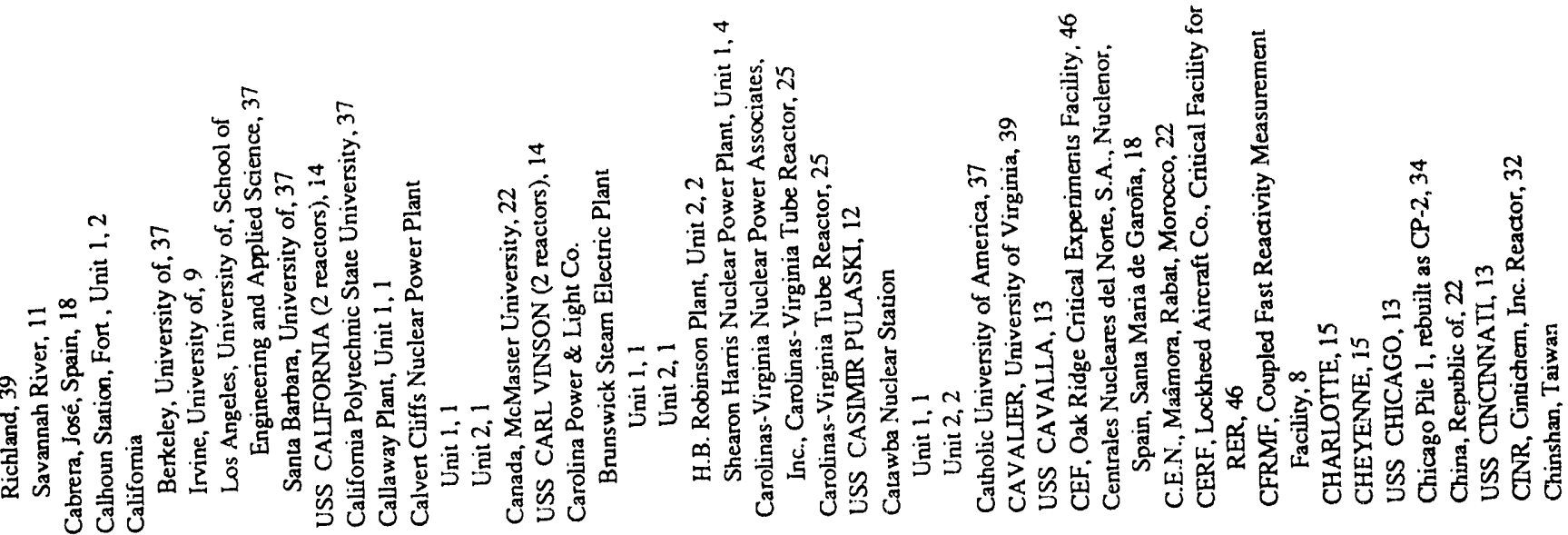

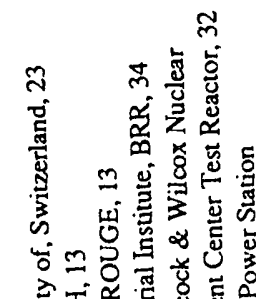

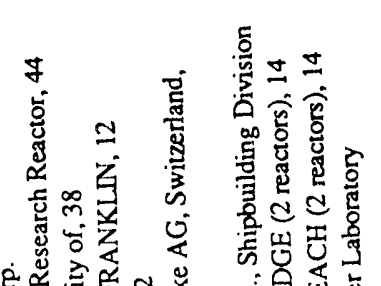

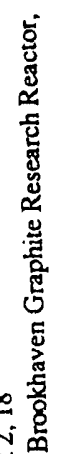

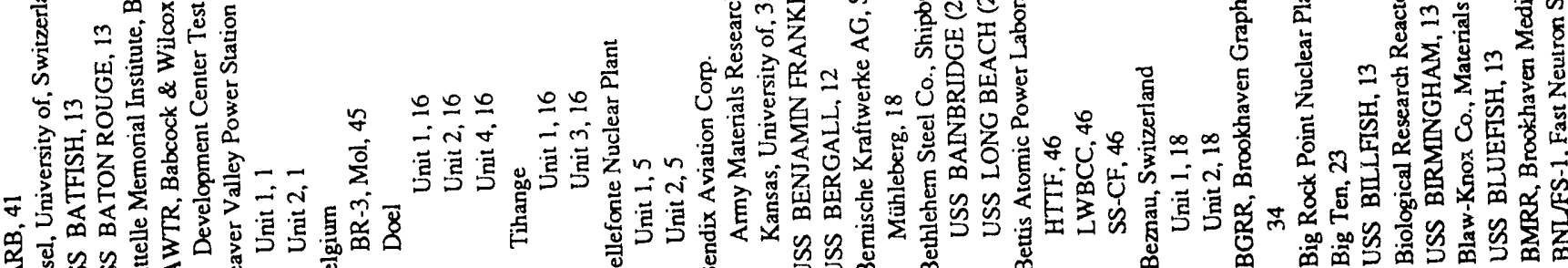

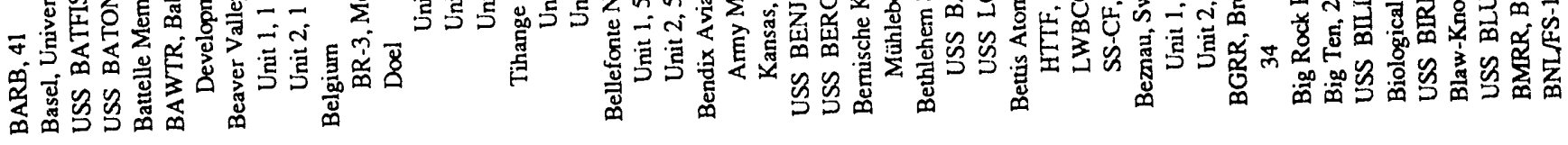

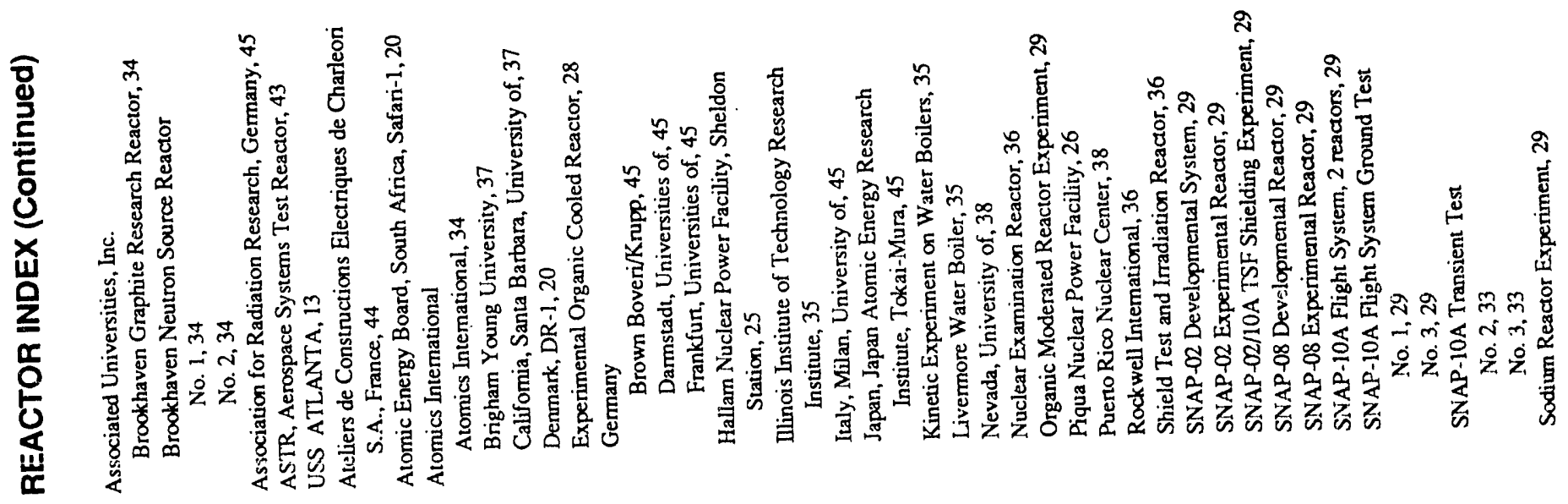



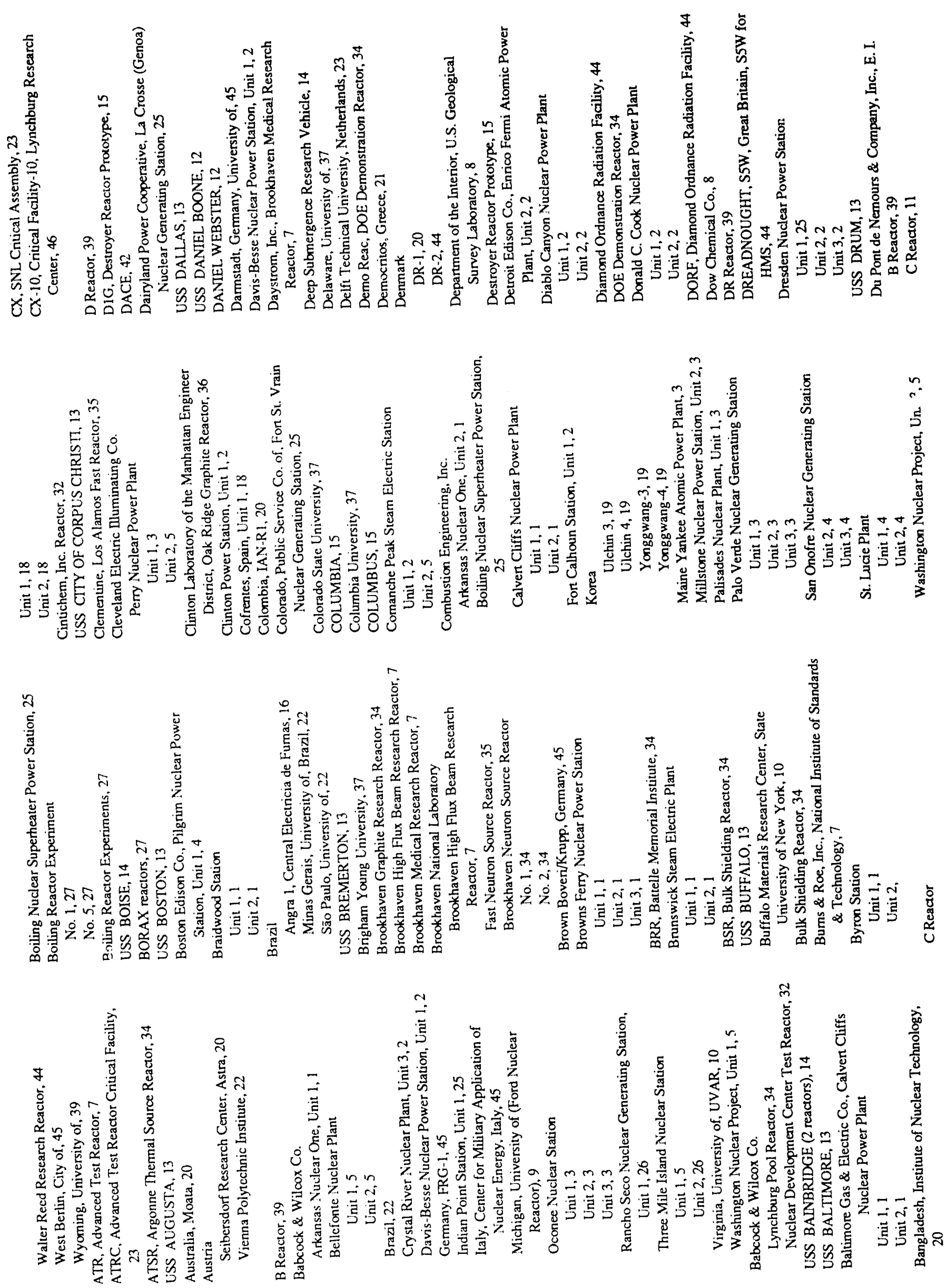

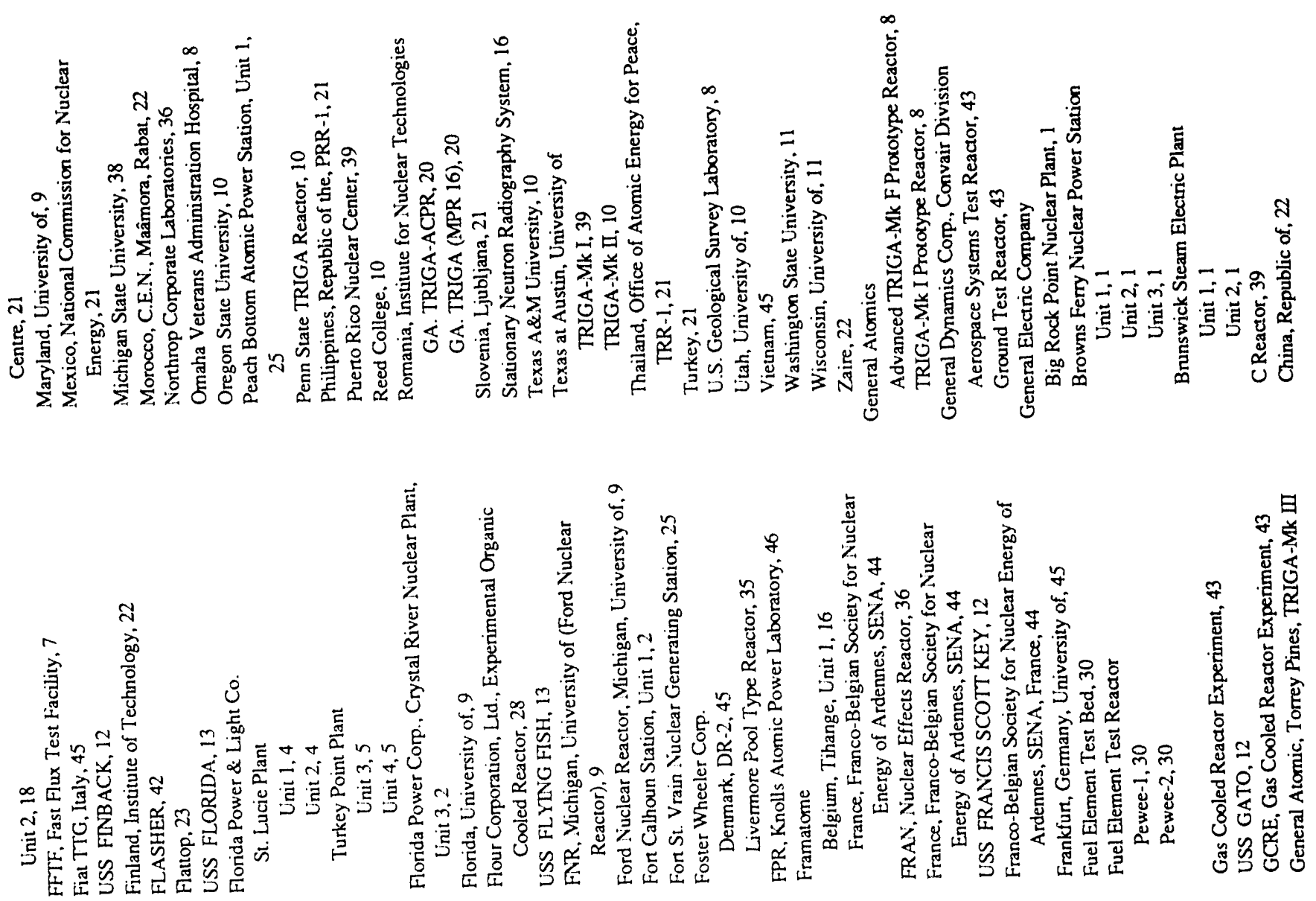

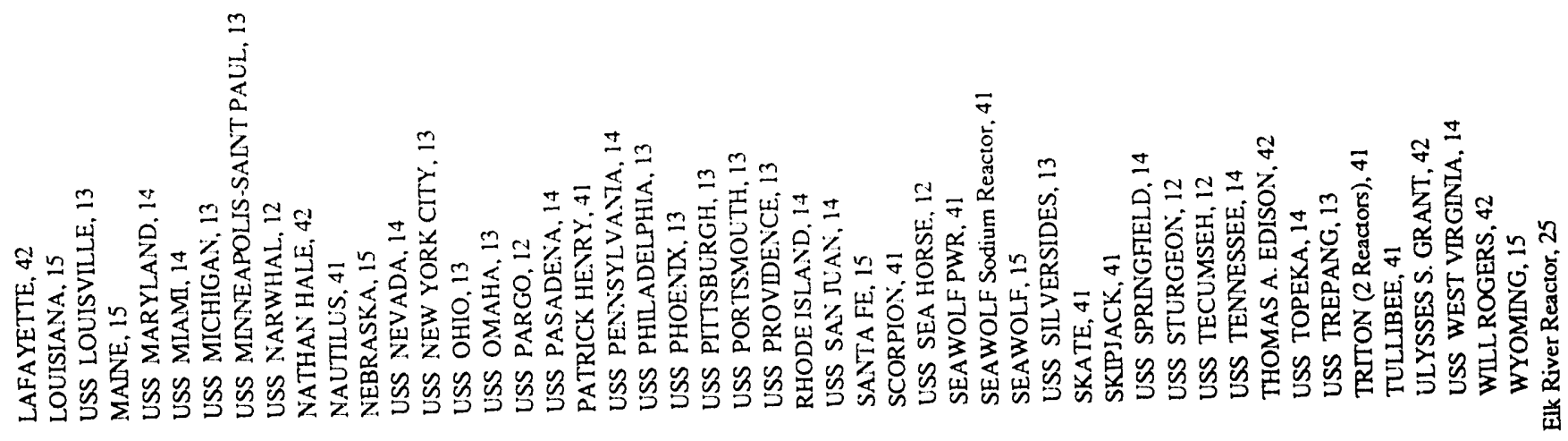

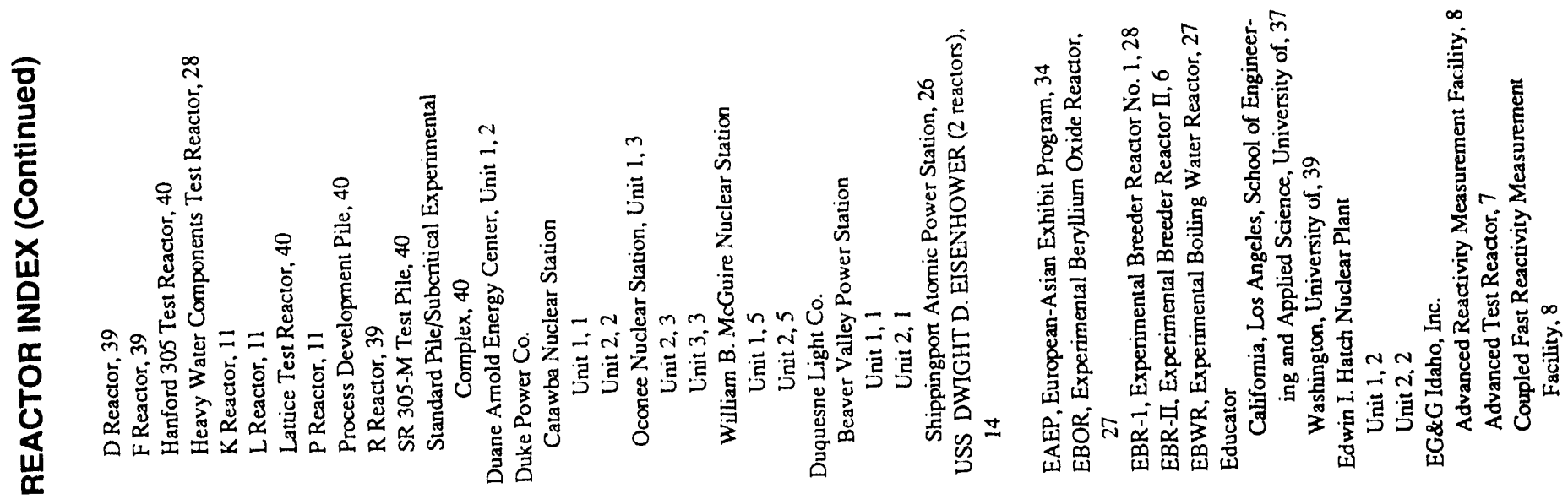



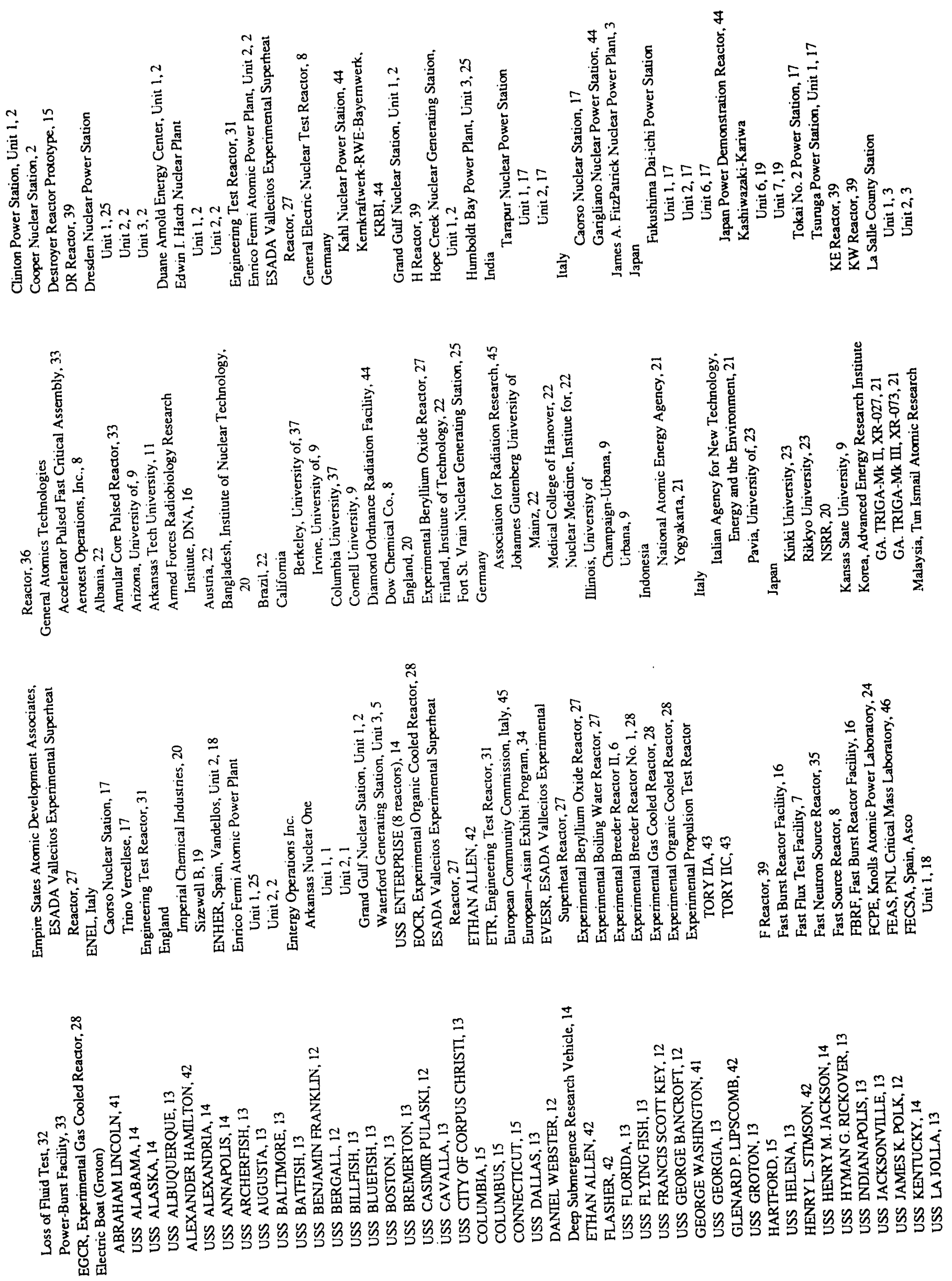

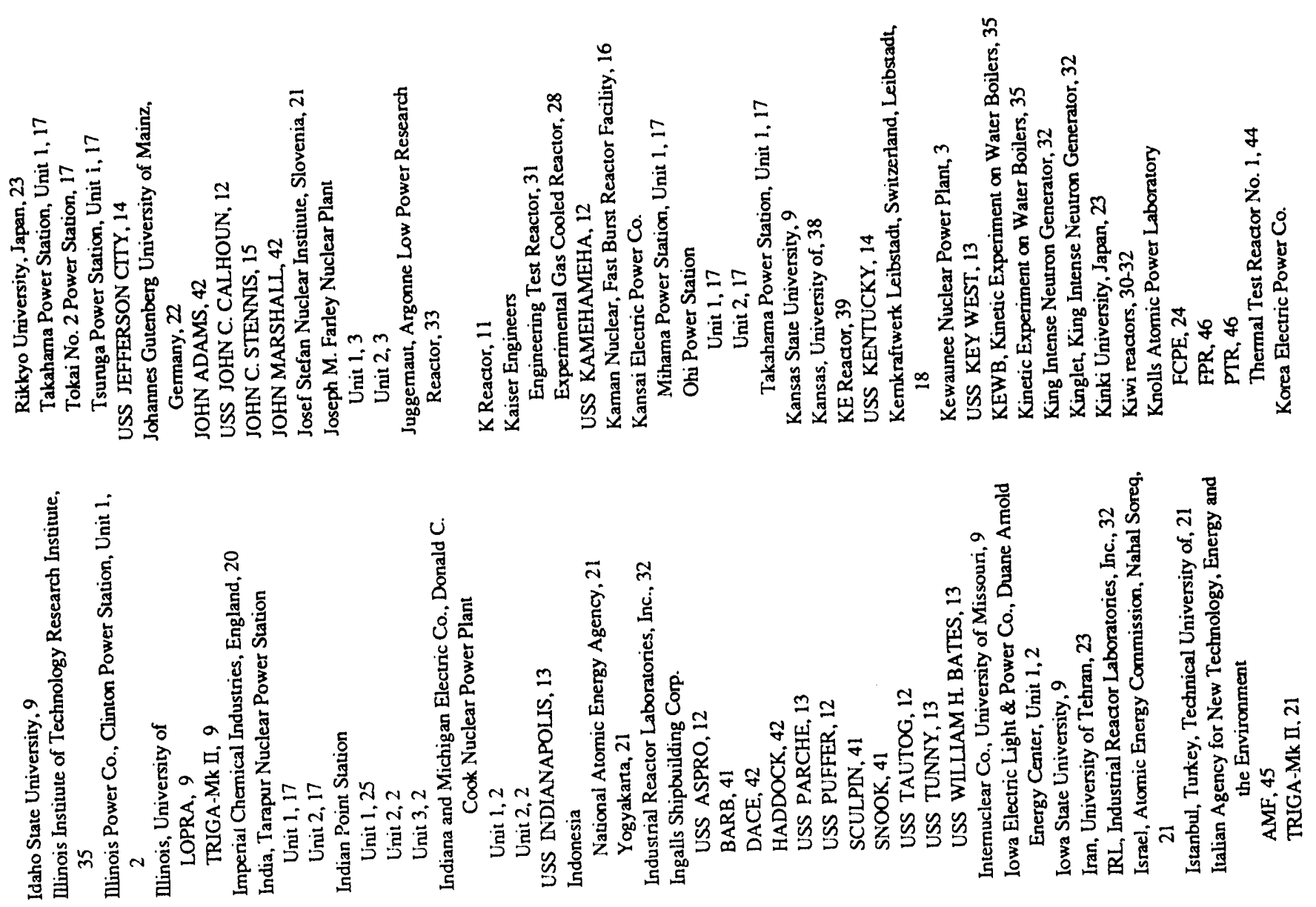

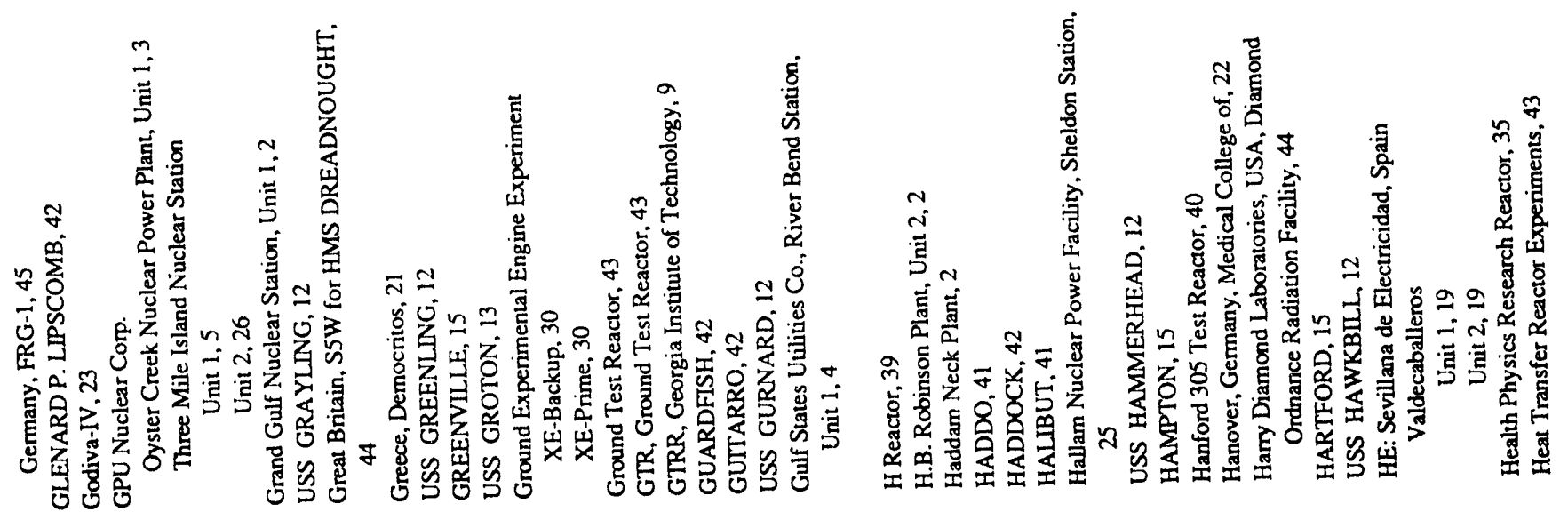

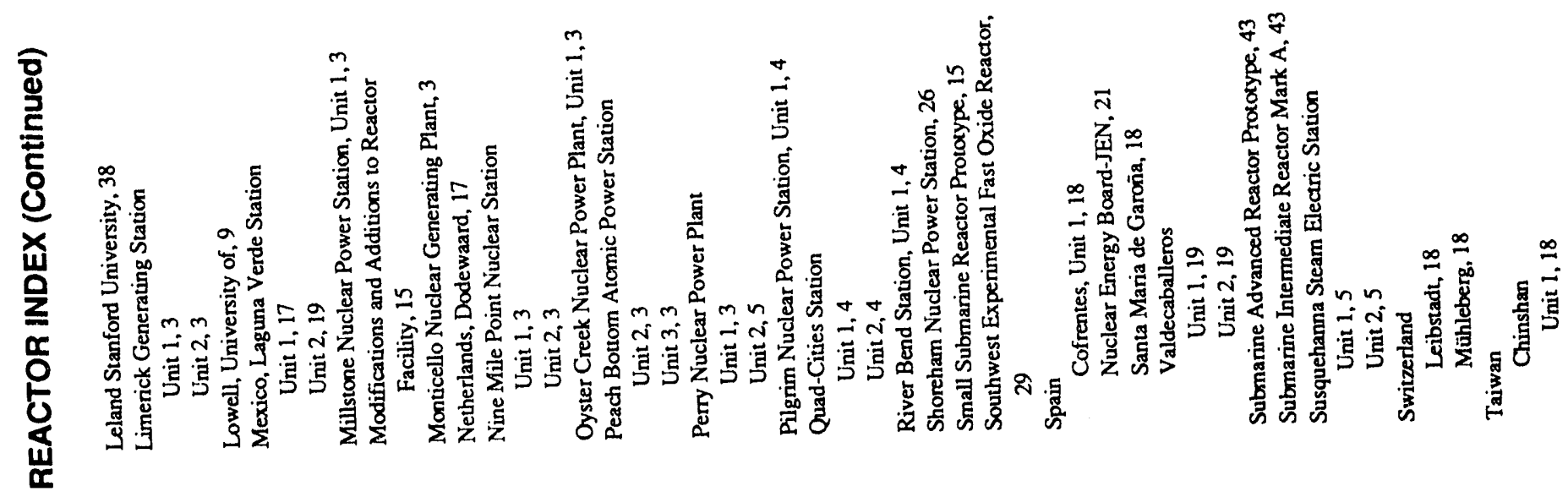



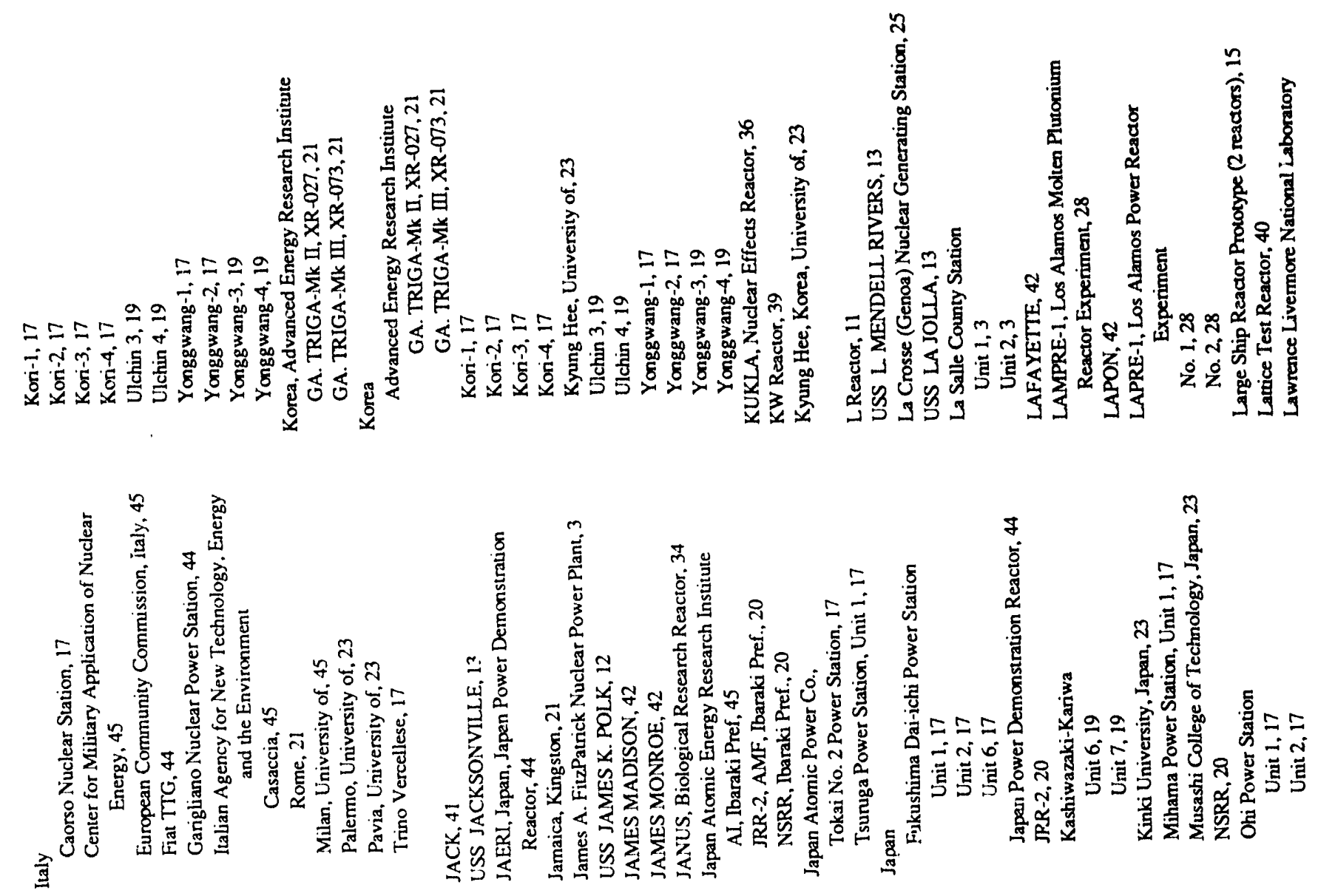

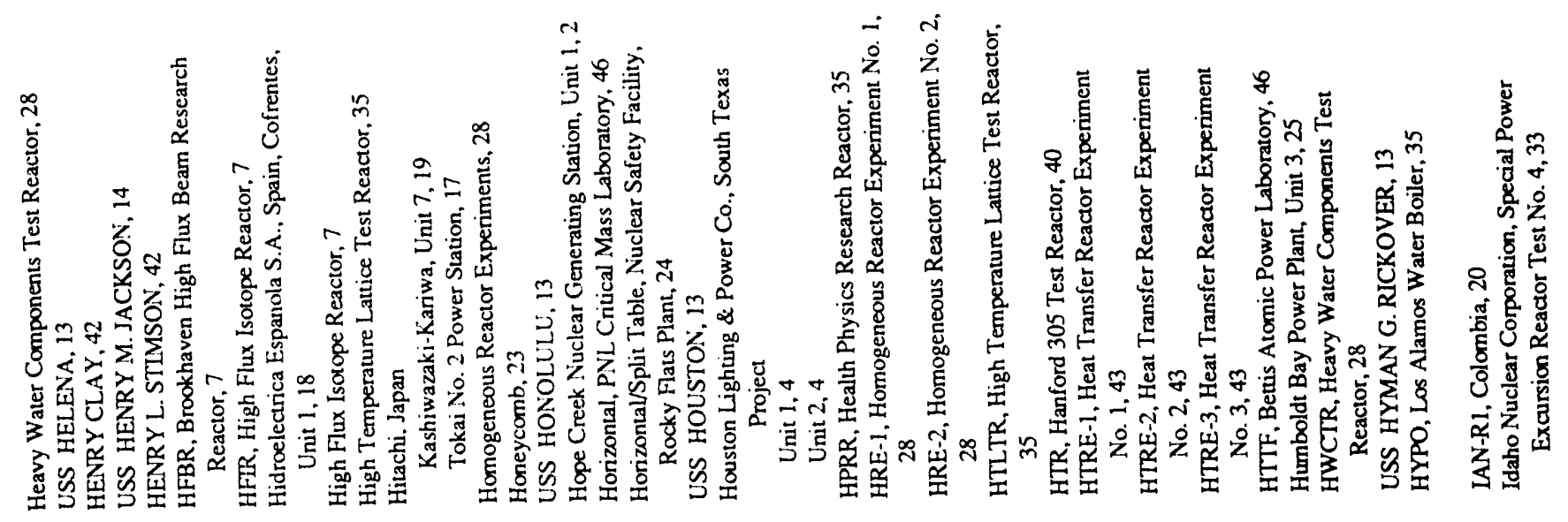
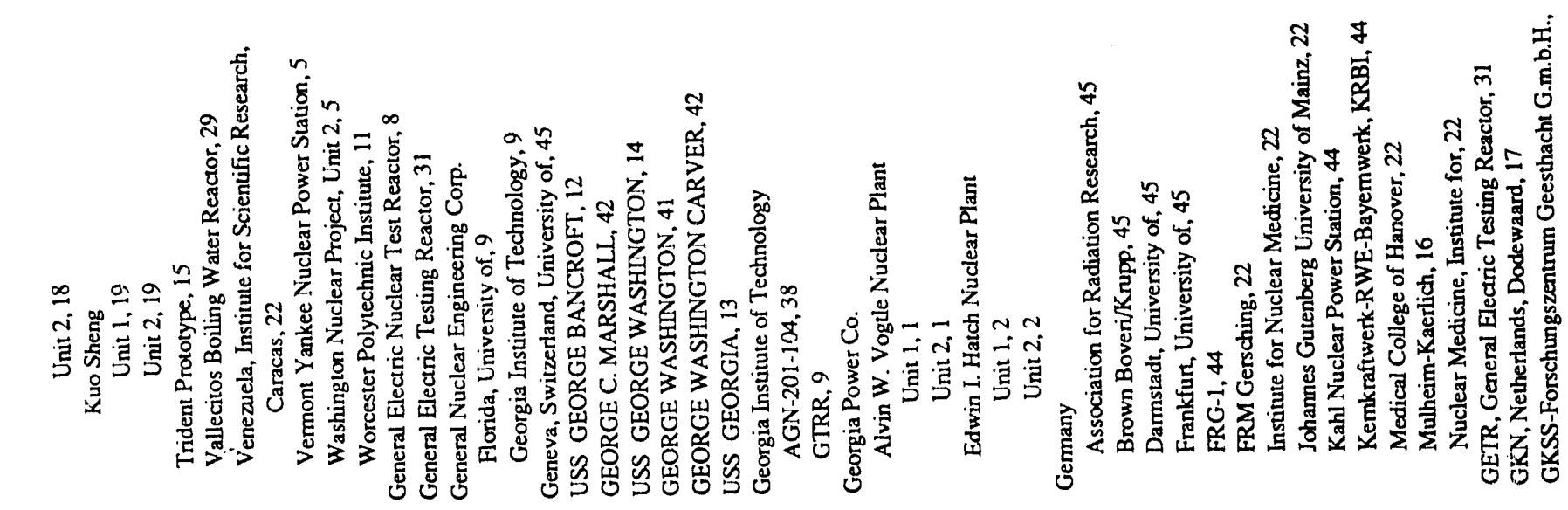

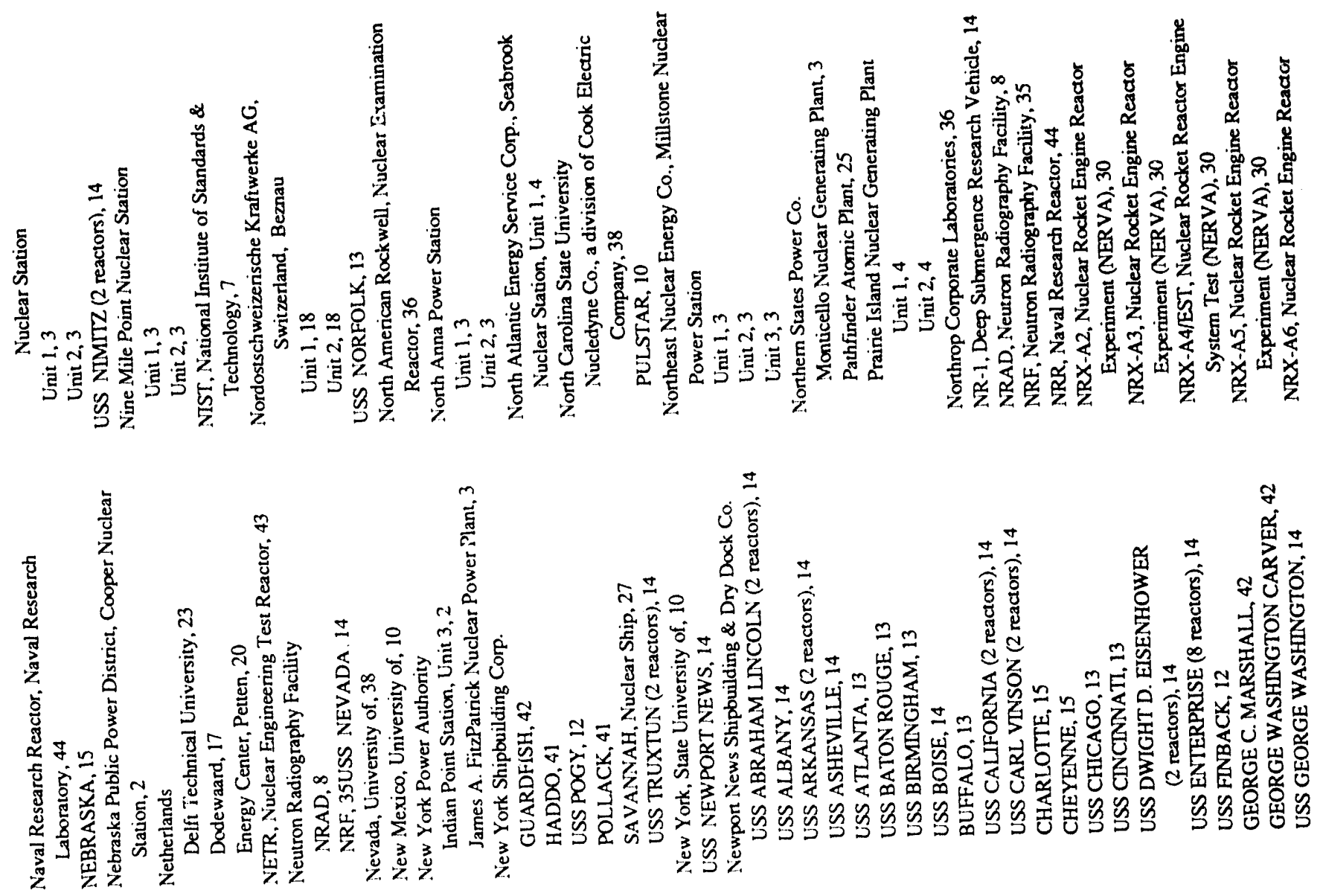

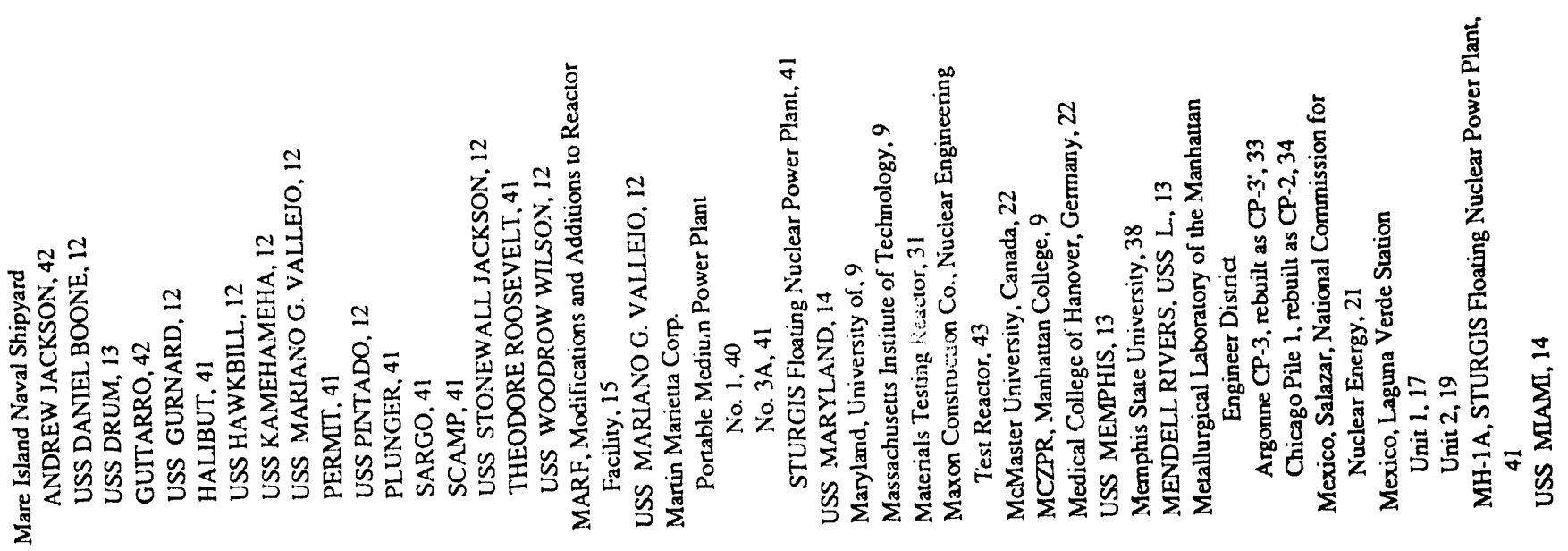

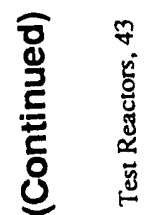

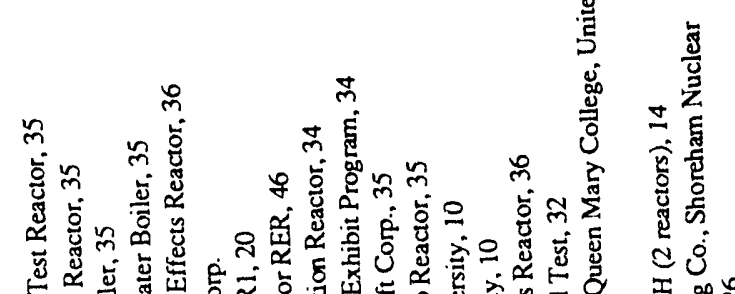

$$
\begin{aligned}
& \text { \%ั }
\end{aligned}
$$

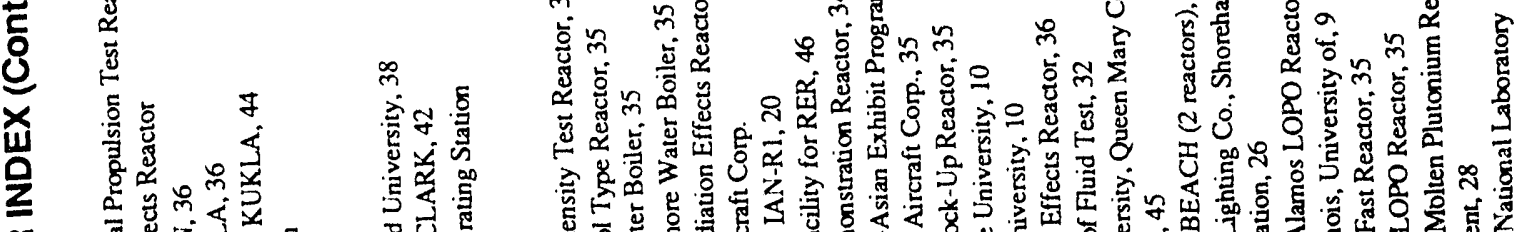

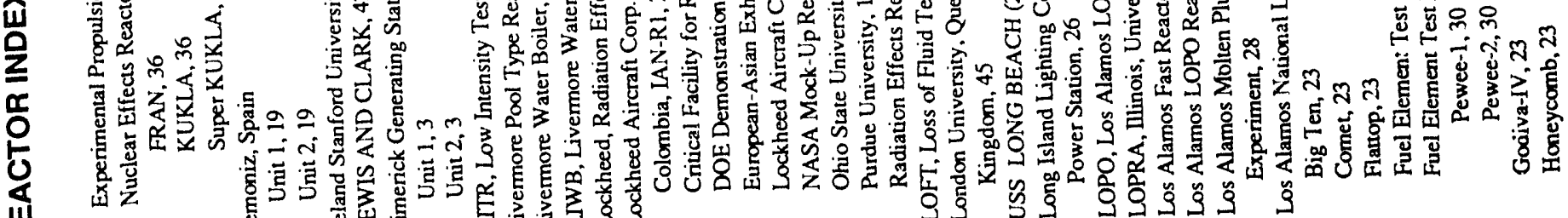



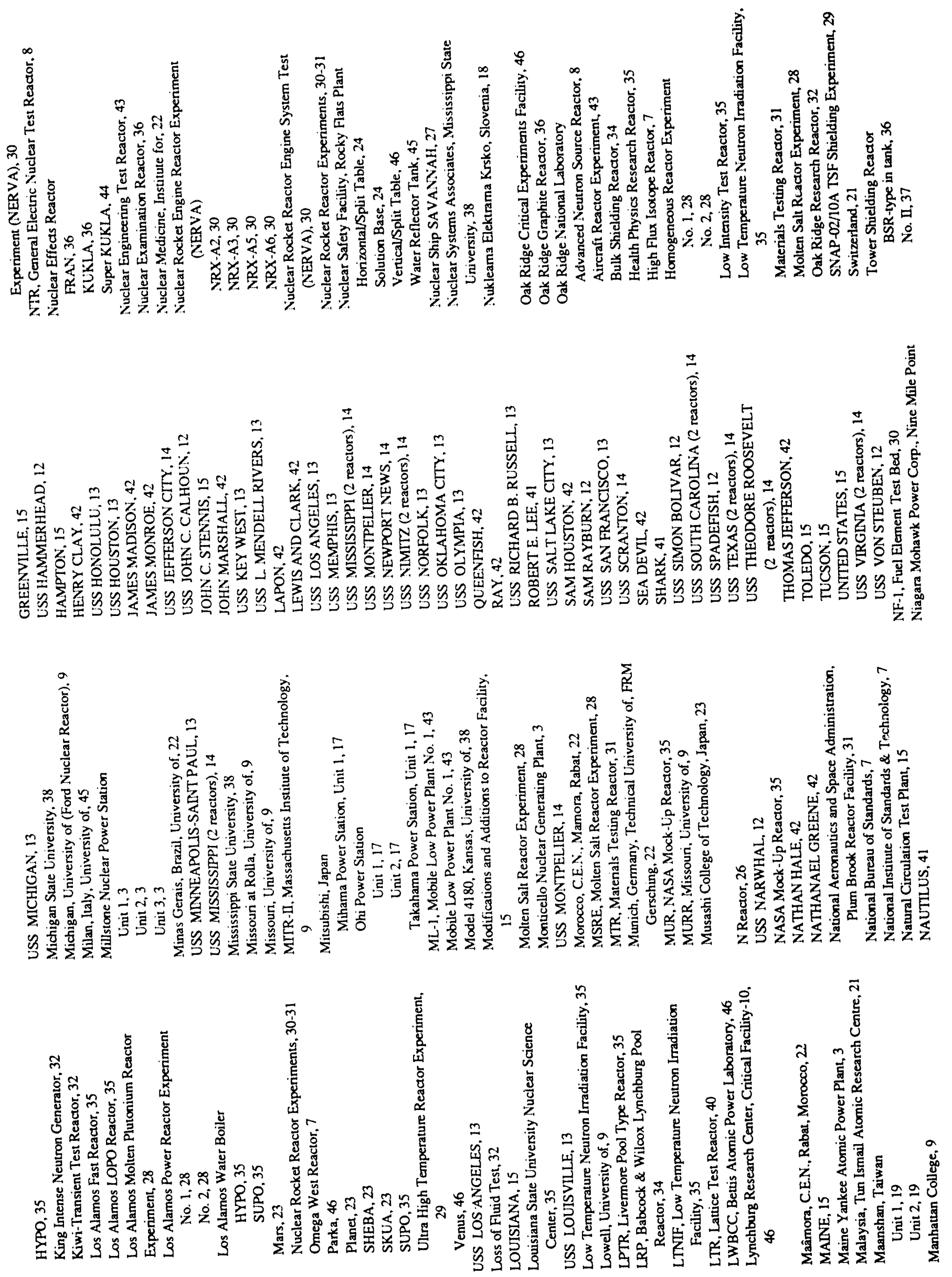

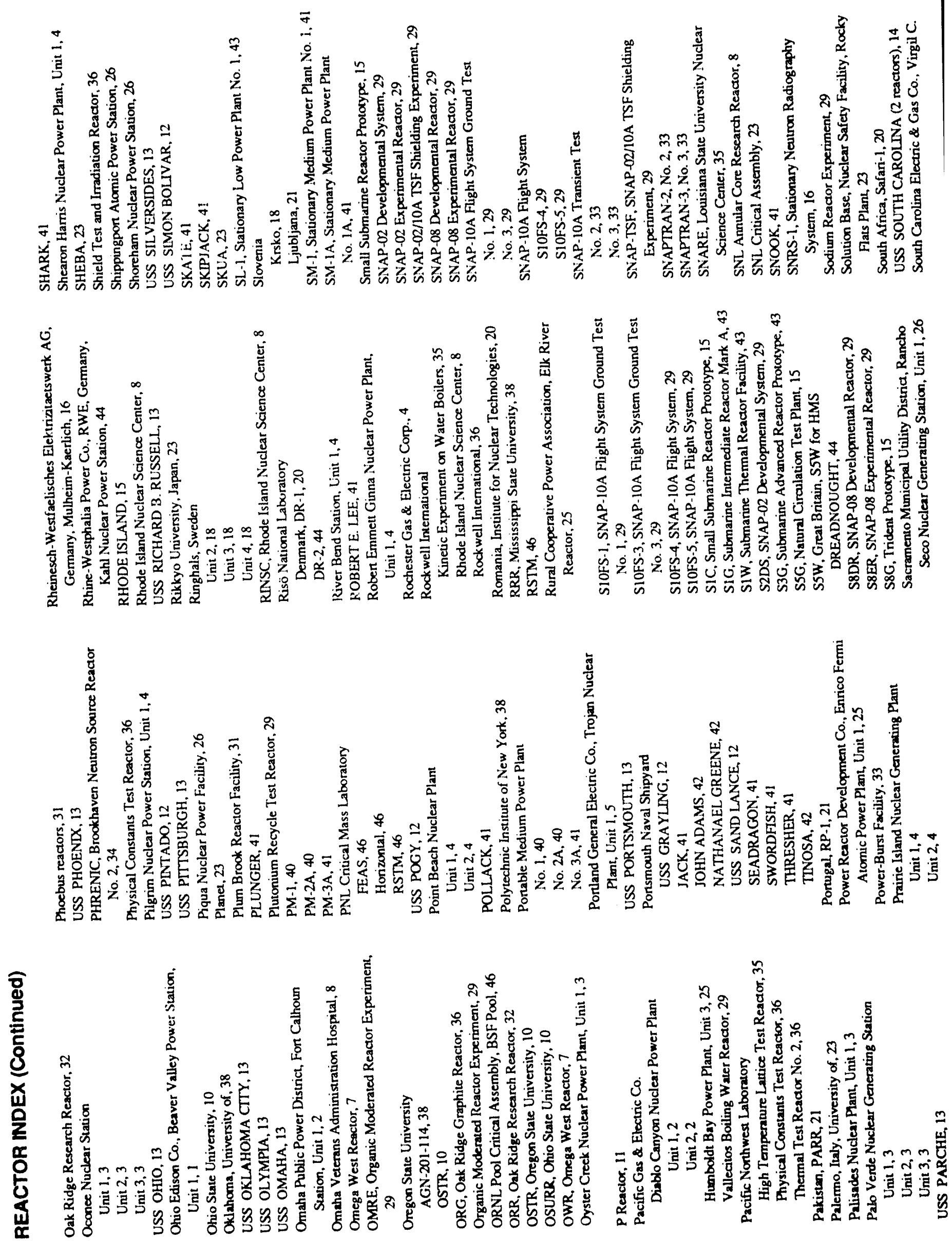


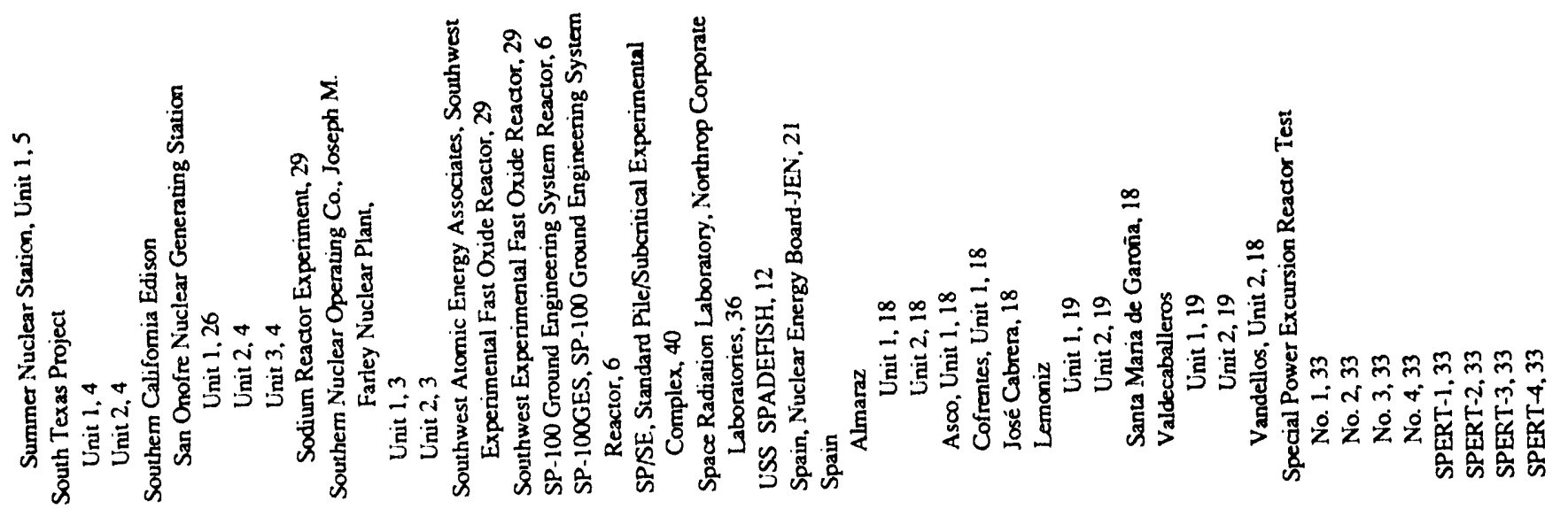

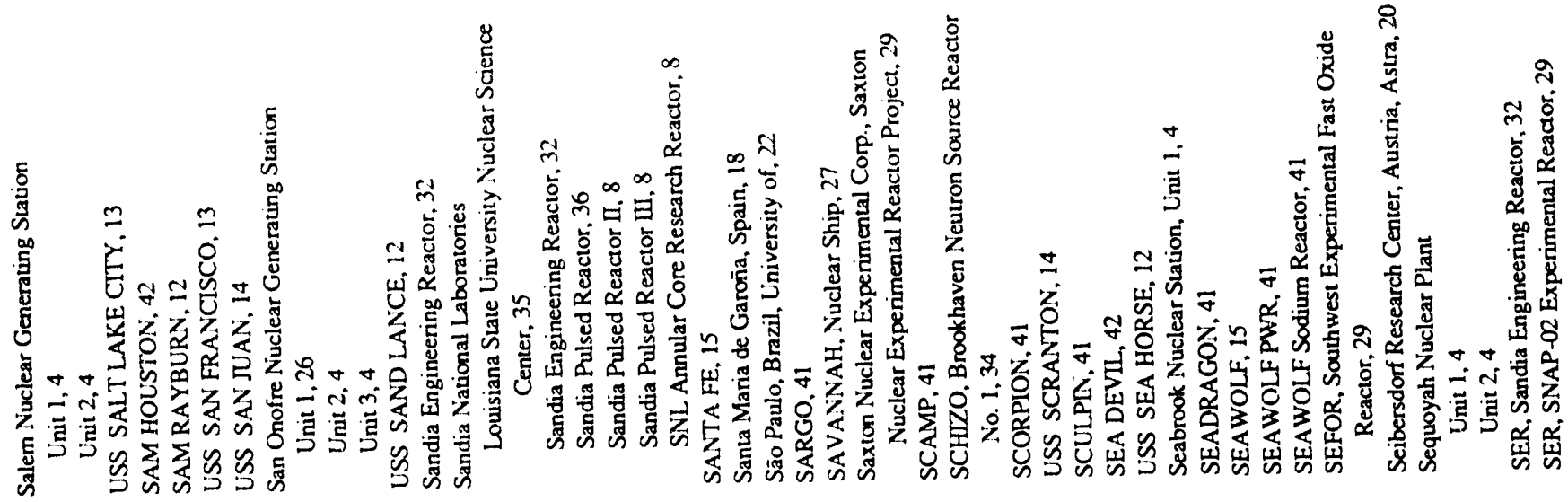
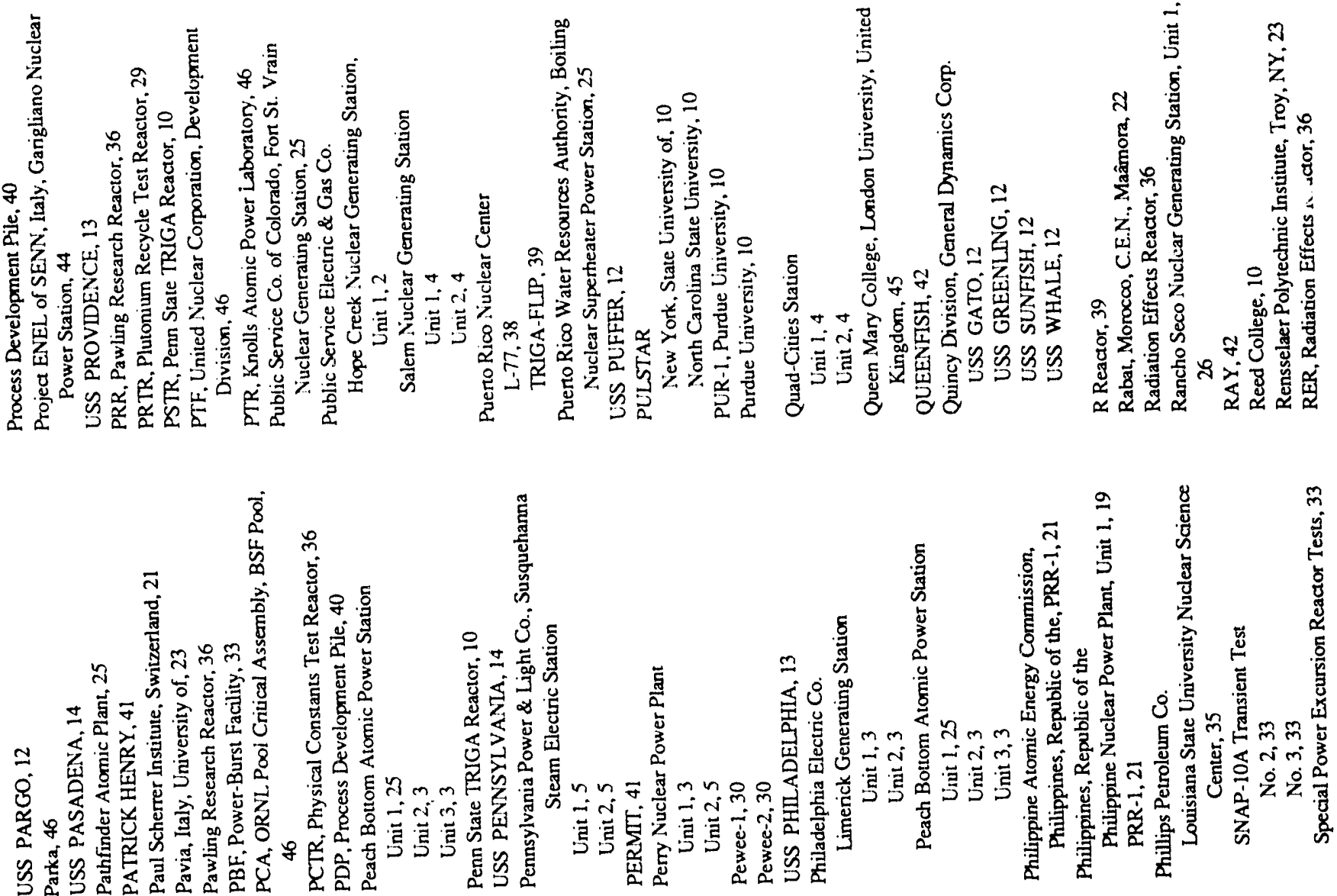

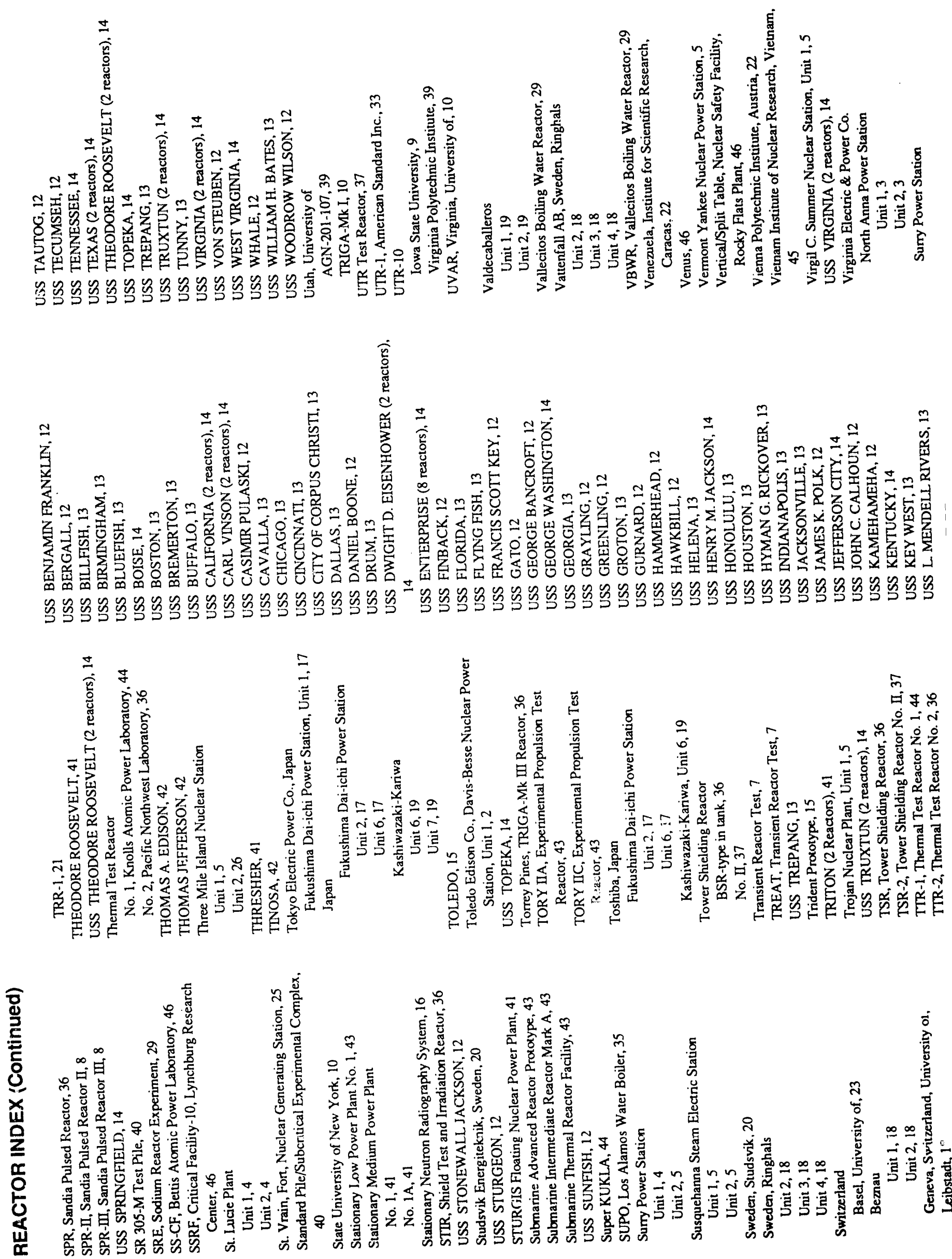


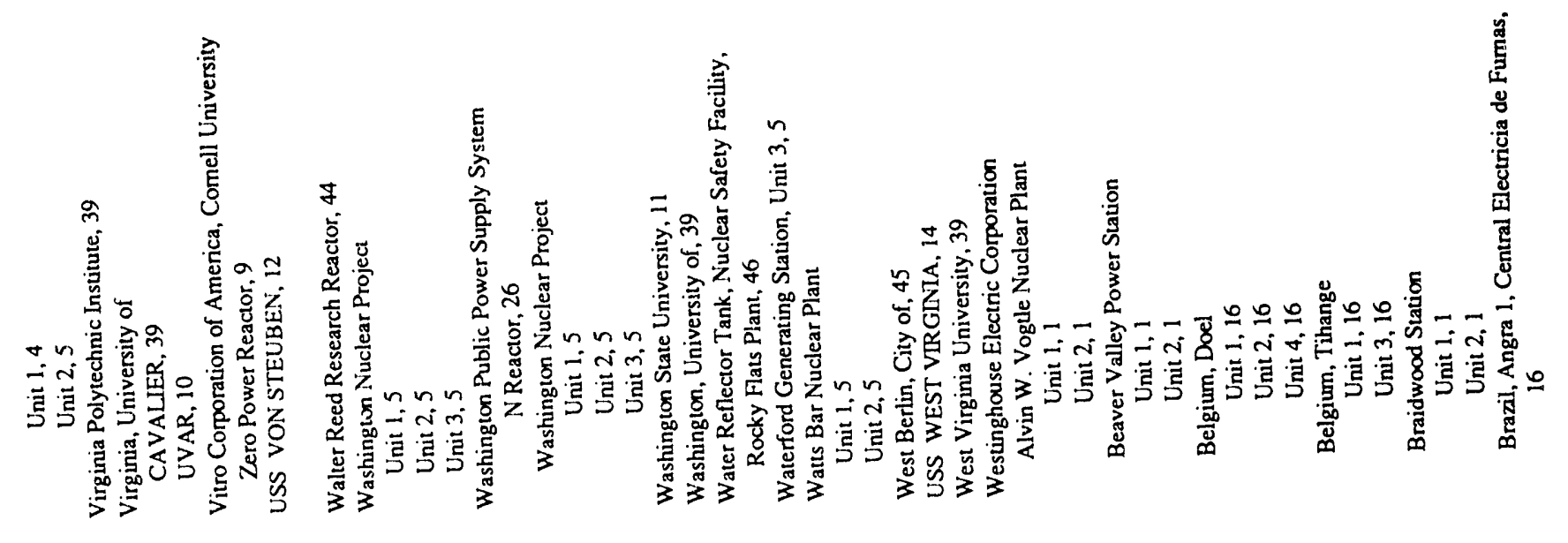

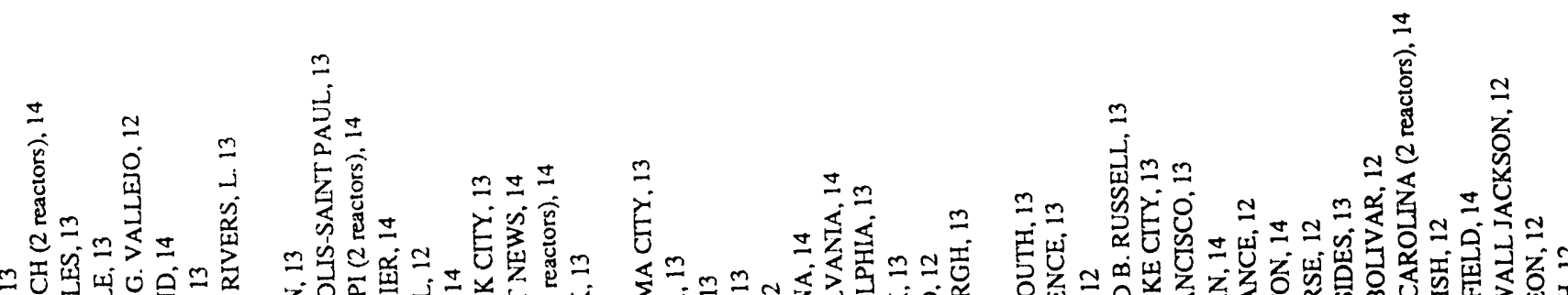

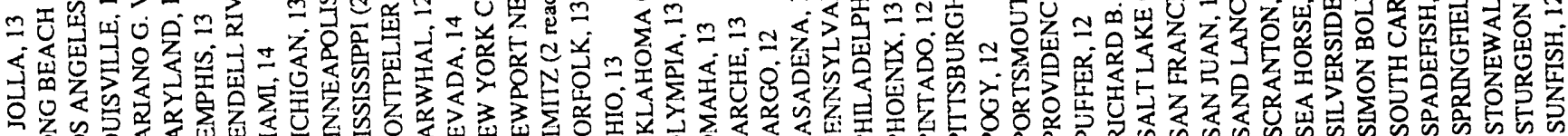

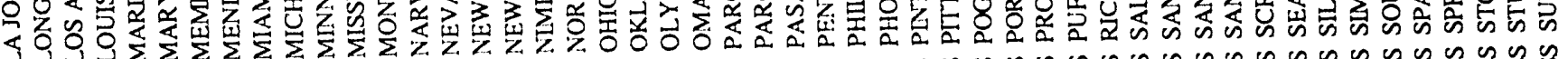

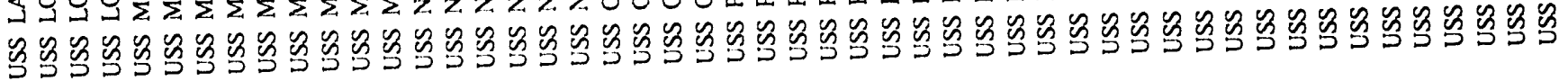

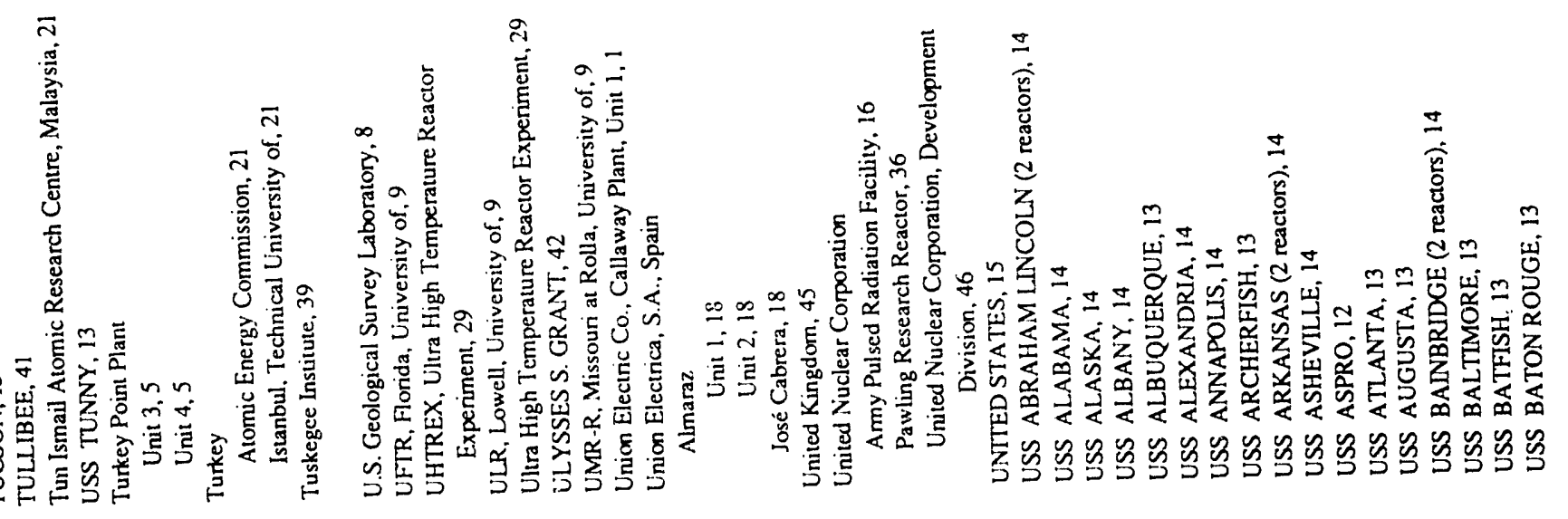

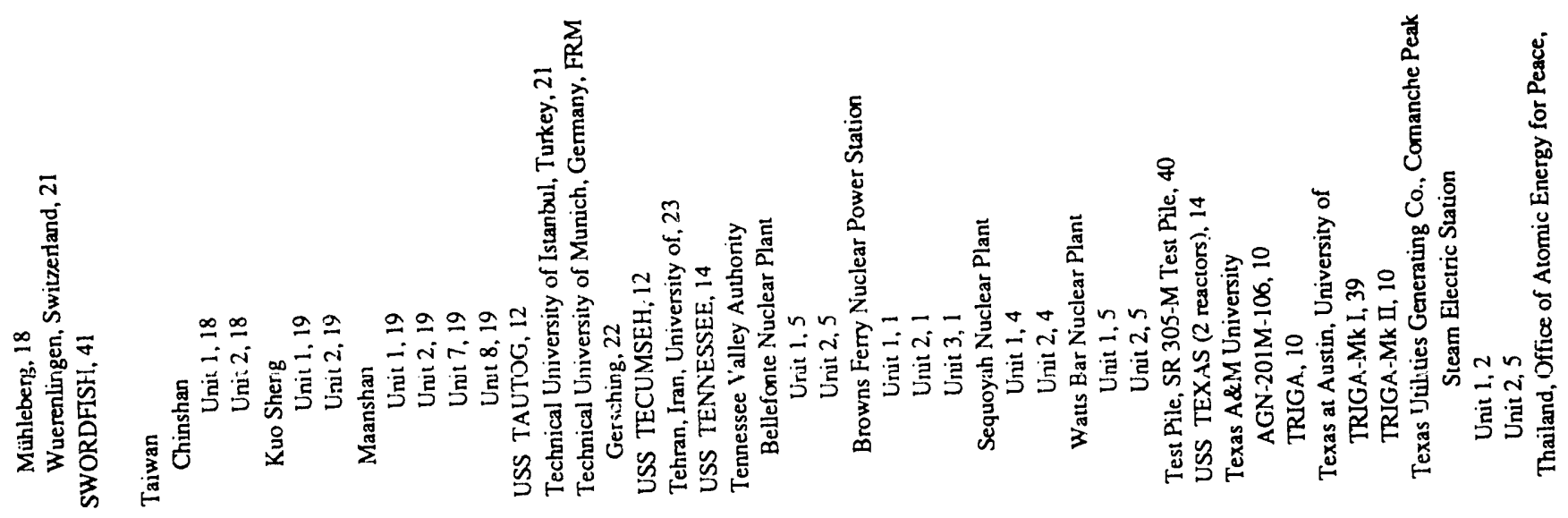




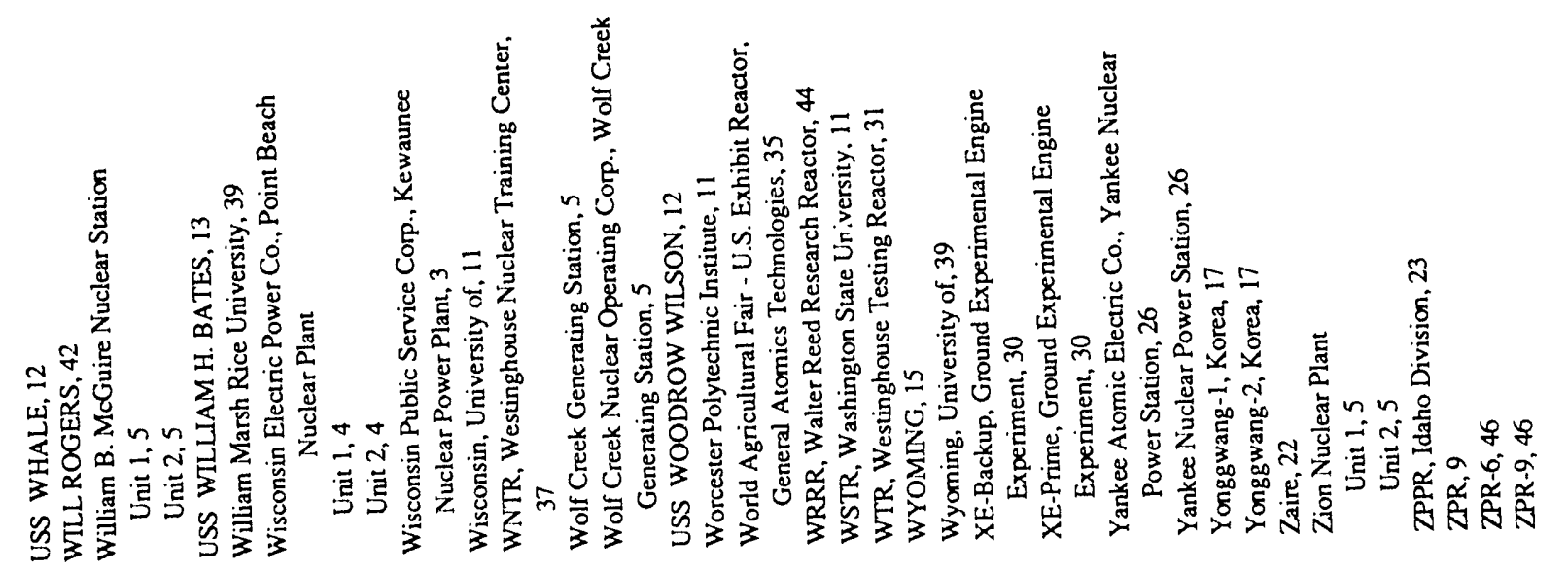

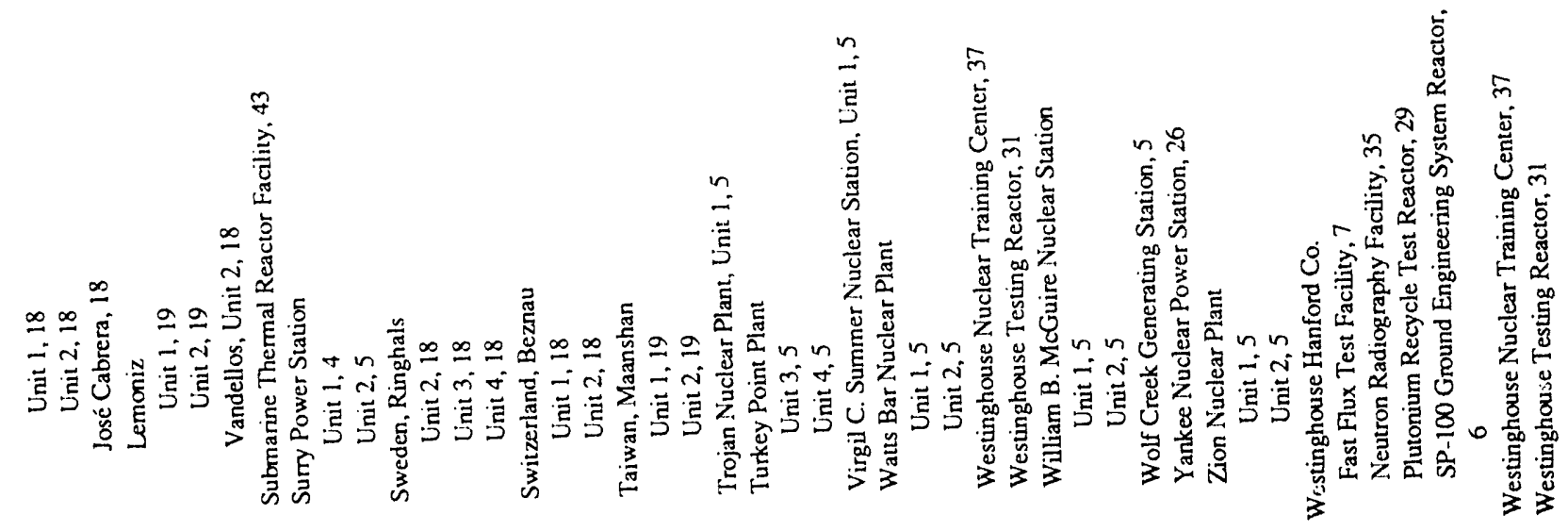
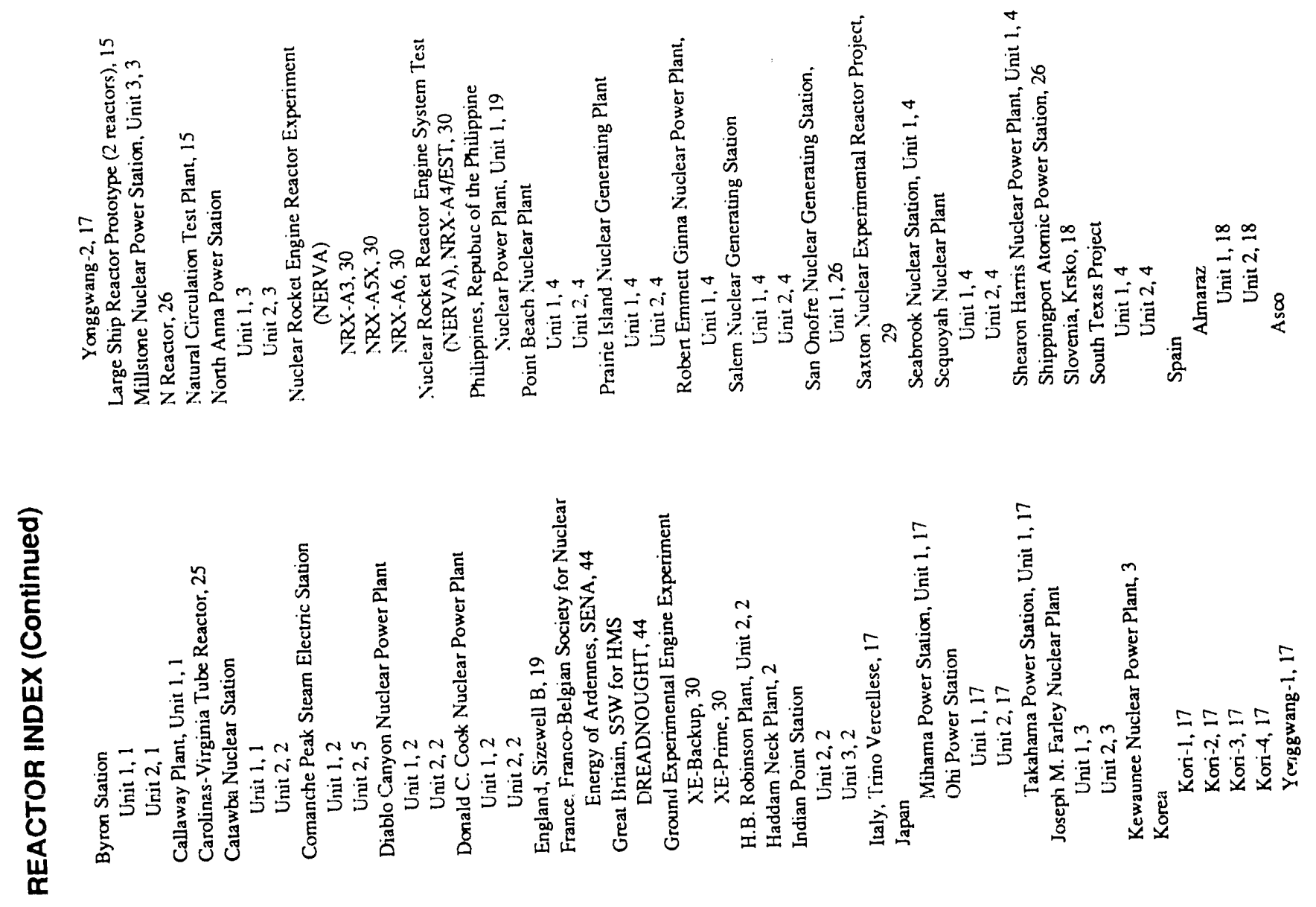

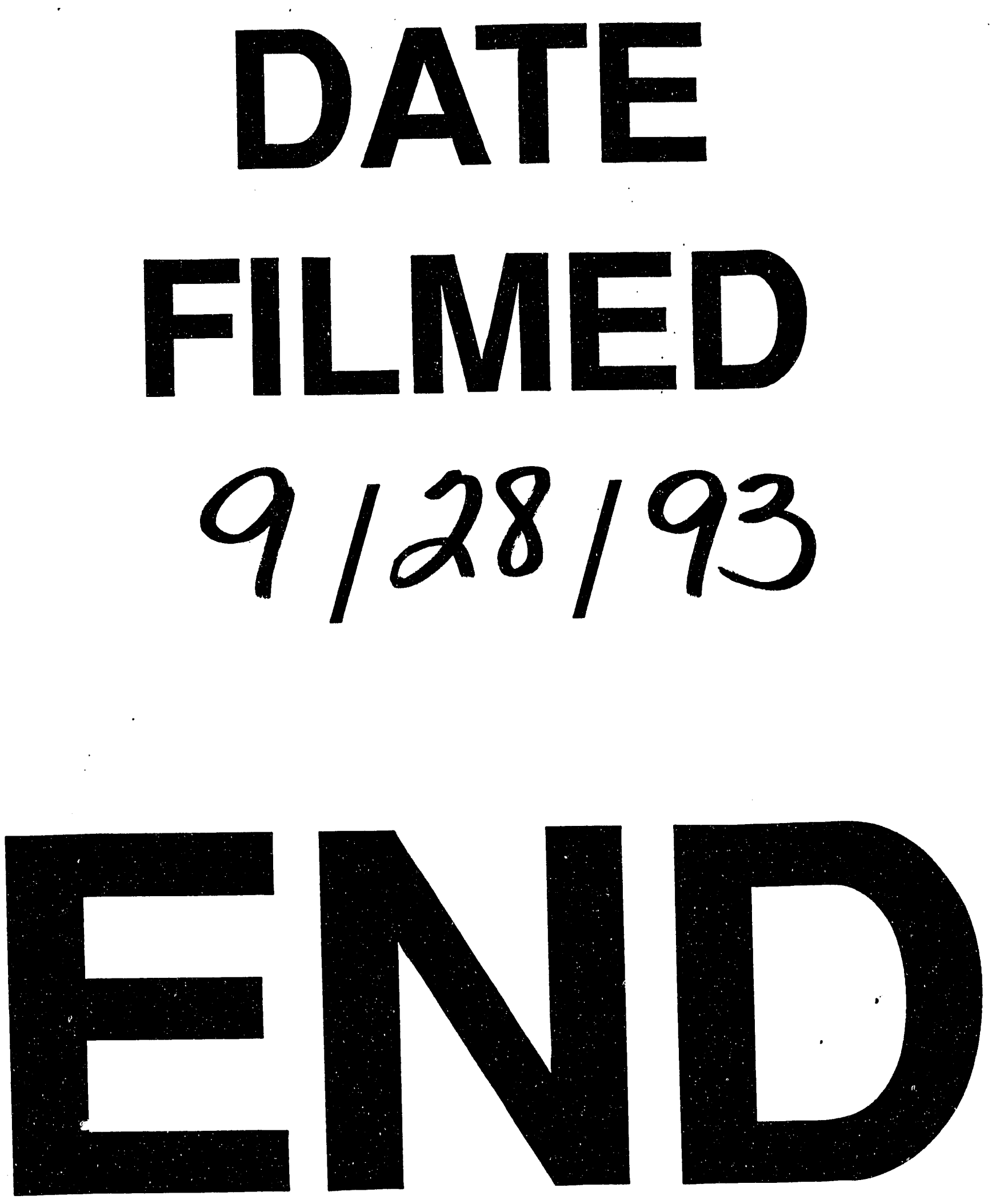
